\title{
THE INFLUENCE OF
}

\section{THERMO-CYCLING AND CYCLICAL LOADING ON METAL/RESIN INTERFACES}

\author{
CONSTANTINOS GEORGIOU \\ KOUNTOURAS \\ BDS University of Liverpool \\ MSc University of London
}

This thesis is submitted in fulfilment of the requirements for the degree of Doctor of Philosophy in the Faculty of Medicine Departments of Prosthetic Dentistry and Biomaterials Science

\section{Eastman Dental Institute}

University of London 
ProQuest Number: 10010092

All rights reserved

\title{
INFORMATION TO ALL USERS
}

The quality of this reproduction is dependent upon the quality of the copy submitted.

In the unlikely event that the author did not send a complete manuscript and there are missing pages, these will be noted. Also, if material had to be removed, a note will indicate the deletion.

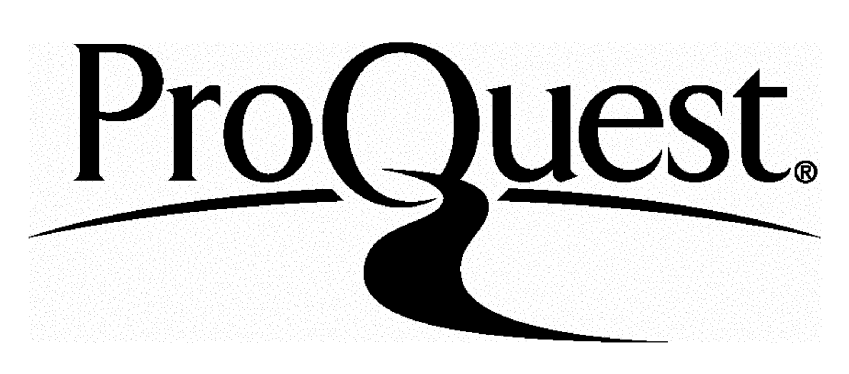

ProQuest 10010092

Published by ProQuest LLC(2016). Copyright of the Dissertation is held by the Author.

All rights reserved.

This work is protected against unauthorized copying under Title 17, United States Code. Microform Edition (c) ProQuest LLC.

\author{
ProQuest LLC \\ 789 East Eisenhower Parkway \\ P.O. Box 1346 \\ Ann Arbor, MI 48106-1346
}




\section{DEDICATED}

\section{TO MY WIFE}

\section{LAURA}

For all her patience and support

AND OUR DAUGHTER

ANASTASIA 


\begin{abstract}
This study investigated the behaviour of metal/resin laminates of dimethacrylate resins and cobalt chromium alloy $(\mathrm{Co} / \mathrm{Cr})$ when subjected to fatigue stressing by thermo-cycling and cyclical loading, after water storage. The veneering materials used were a microfine (Silux Plus) and a hybrid (Z100) composite, bonded to a $\mathrm{Co} / \mathrm{Cr}$ alloy through an adhesive interface (Cesead opaque primer and body opaque resin). Characterisation of the two composite resins was carried out with particular attention to water sorption. Laminates were evaluated over a period up to six months, groups of ten specimens were load cycled alone (Ld) (up to 453,600 cycles at $5 \mathrm{~Hz}$ ), thermo-cycled alone (Th) (up to 25,200 cycles between $4^{\circ} \mathrm{C}, 37^{\circ} \mathrm{C}$ and $60^{\circ} \mathrm{C}$ ) load cycled and thermo-cycled (Ld/Th) (cycled as above). Following testing, laminates were assessed for their elastic modulus, examined microscopically and the adhesive interface was subjected to a dye penetration study. The microfine resin absorbed more water than the hybrid $(2.88 \%$ and $1.84 \%$ by mass respectively) and lost more soluble material $(0.61 \%, 0.19 \%$ of original mass respectively). The laminates of the different veneering resins exhibited differences in their elastic behaviour. The apparent flexural modulus of laminates made with the hybrid resin (initial: $482.3 \pm 69.1 \mathrm{GPa}$, week $24 \mathrm{Ld} / \mathrm{Th}: 544.7 \pm 70.3 \mathrm{GPa}$ ) was higher than those made with the microfine resin (initial: $288.1 \pm 44.4 \mathrm{GPa}$, week $24 \mathrm{Ld} / \mathrm{Th}: 353.7 \pm 47.5 \mathrm{GPa}$ ). The extension at failure of the hybrid resin laminates appeared to be lower than that of the microfine ones. However, little difference was seen in the stress at failure between groups. Week $24 \mathrm{Ld} / \mathrm{Th}$ : (Z100: $833.3 \pm 355.8 \mathrm{MPa}$, Silux Plus: $828.4 \pm 122.1 \mathrm{MPa})$. Both cohesive failure within the veneering resin and adhesive failures between the veneering resin and metal component were noted.
\end{abstract}




\section{TABLE OF CONTENTS}

\section{ABSTRACT}

TABLE OF CONTENTS I

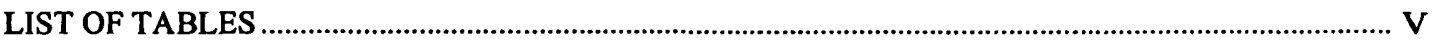

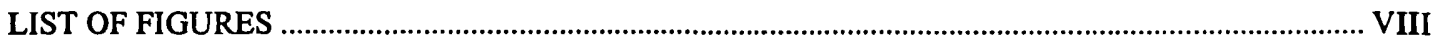

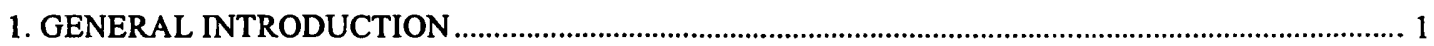

1.1 THE NEED FOR OVERLAY REMOVABLE PARTIAL DENTURES ….................................. 1

1.2 FAILURE MODES IN METAL BASED OVERLAY REMOVABLE PARTIAL DENTURES ....... 2

1.3 PREDISPOSING FACTORS PRECIPITATING OVERLAY FAILURES ..................................... 2

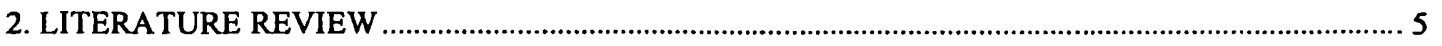

2.1 MATERIALS USED FOR THE MANUFACTURE OF OVERLAY REMOVABLE PARTIAL

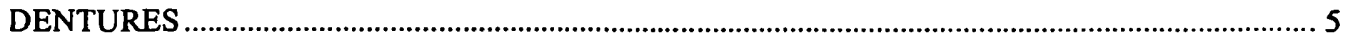

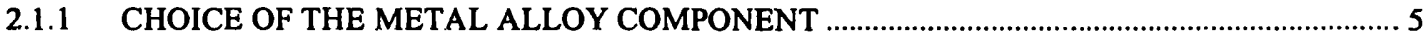

2.1.2 CHOICE OF THE VENEERING COMPONENT …................................................................. 10

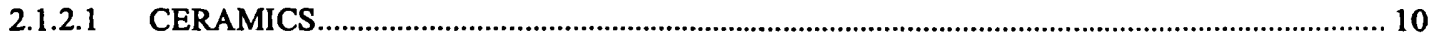

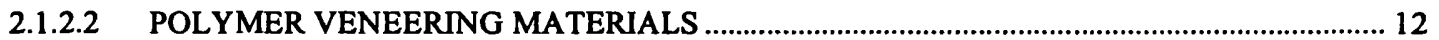

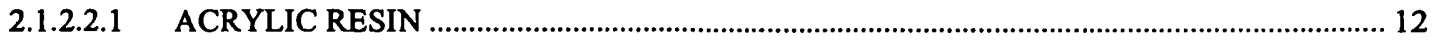

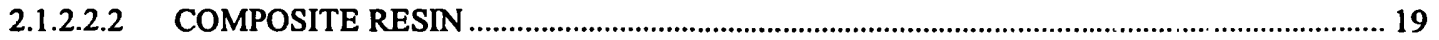

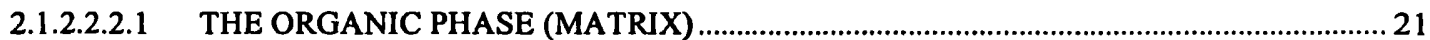

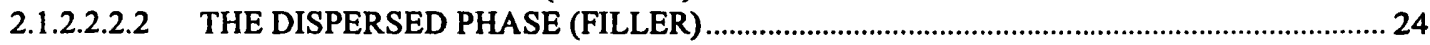

2.1.2.2.2.3 THE INTERFACIAL PHASE (COUPLING AGENT) .................................................. 29

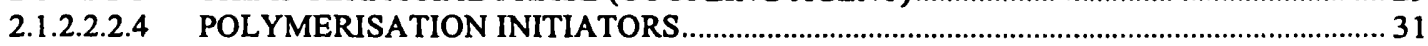

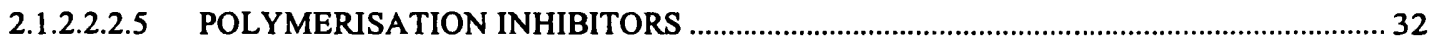

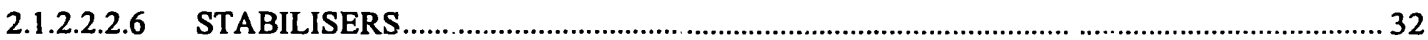

2.2 DEVELOPMENTS IN THE POLYMERISATION OF COMPOSITE RESINS ............................. 32

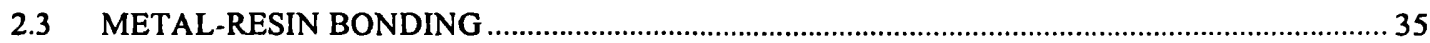

2.3.1 MECHANICAL RETENTION

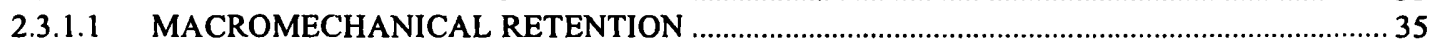

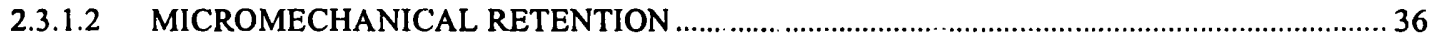

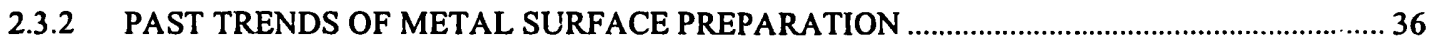

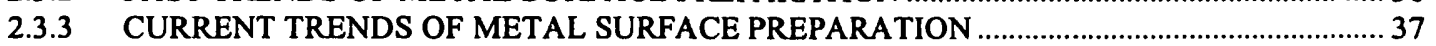

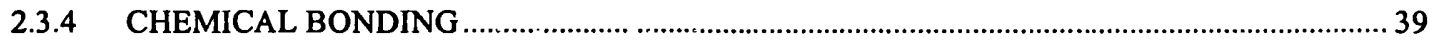

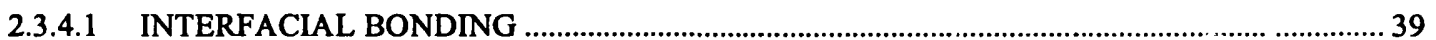

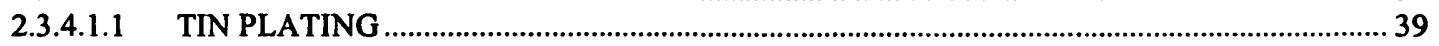

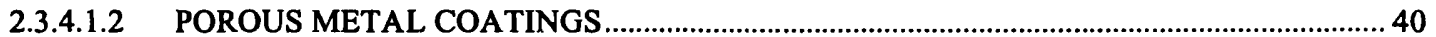

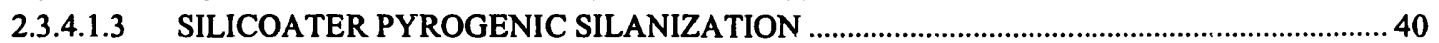

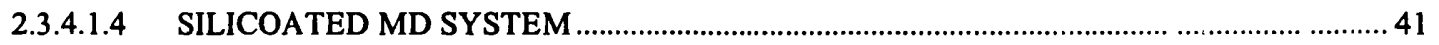

2.3.4.1.5 ROCATEC SYSTEM

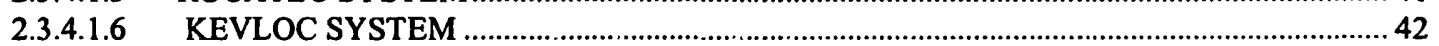

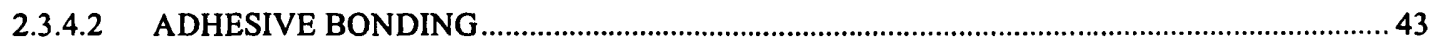

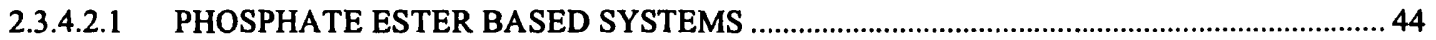

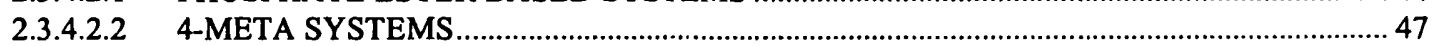

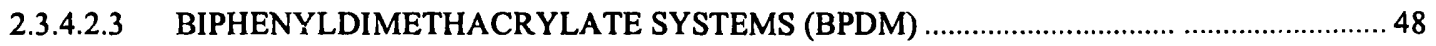

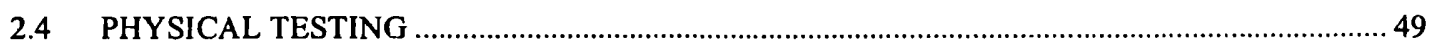

2.4.1 EVALUATION OF DIFFERENT TESTING CONDITIONS .............................................. 49

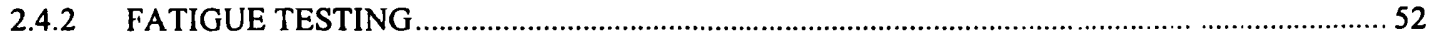

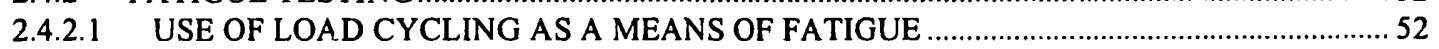




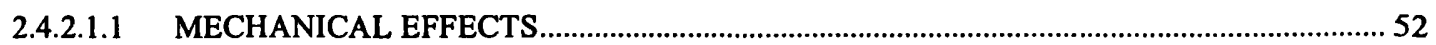

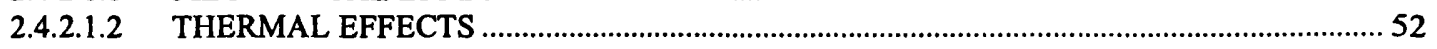

2.4.2.2 CRACK FORMATION AND PROPAGATION …………………………………………....5 53

2.4.2.3 USE OF THERMO-CYCLING AND WATER SORPTION AS A MEANS OF FATIGUE...... 55

2.4.3 FLEXURAL TESTING .................................................................................................... 59

2.5 EFFECT OF LIGHT POLYMERISATION SOURCE AND POLYMERISATION SHRINKAGE 60

2.6 CURRENT STATUS OF COMPOSITE MATERIALS ...................................................................62

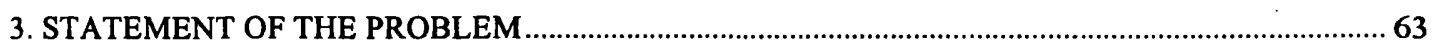

3.1 THE PROBLEM

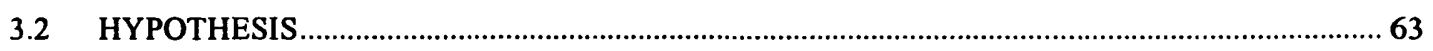

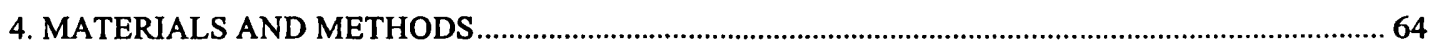

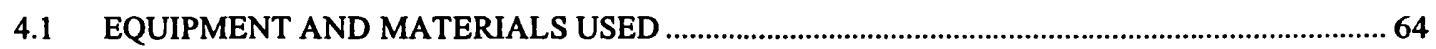

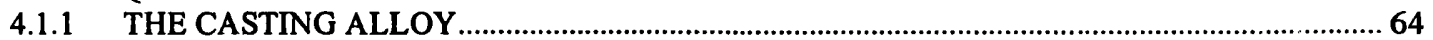

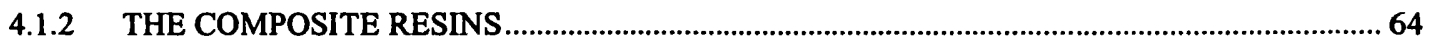

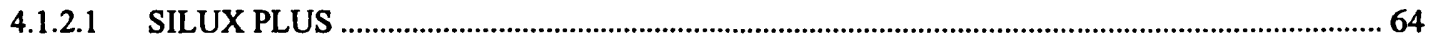

4.1.2.2 Z100

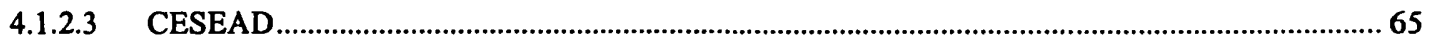

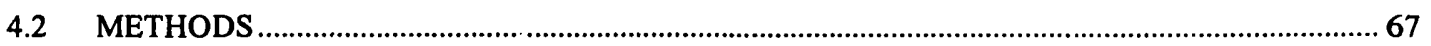

4.2.1 METHOD TO ASSESS THE MECHANICAL PROPERTIES OF THE COMPONENTS ...........67 67

4.2.1.1 MANUFACTURE OF THE METAL COMPONENT OF THE LAMINATE SPECIMENS ..... 67

4.2.1.2 MANUFACTURE OF THE RESIN SPINE SPECIMENS …………………………………....6 68

4.2.1.3 METHOD TO EVALUATE THE SORPTION BEHAVIOUR OF THE RESINS ...................... 71

4.2.1.3.1 MEASUREMENT OF VARIATION IN WEIGHING ........................................................ 74

4.2.1.3.2 FIRST SORPTION CYCLE ……………………………………………………...... 74

4.2.1.3.3 SECOND SORPTION CYCLE ……………………………………………………... 75

4.2.2 MAIN THERMAL AND FLEXURAL LOADING STUDY …………………………………..... 76

4.2.2.1 SPECIMEN PREPARATION FOR THE MAIN THERMAL AND FLEXURAL LOADING

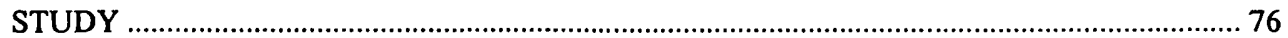

4.2.2.1.1 PREPARATION OF THE METAL COMPONENT ……………………………………...... 76

4.2.2.1.2 PREPARATION OF THE RESIN COMPONENT.................................................................. 77

4.2.2.1.3 FINISHED SPECIMEN HANDLING AND CREATION OF THE TESTING GROUPS......... 80

4.2.2.1.4 THREE POINT BEND TEST OF THE BONDED SPECIMENS (IMMEDIATE GROUP) ... 88

4.2.2.2 DETERMINATION OF THE TESTING CONDITIONS …………………………..................... 89

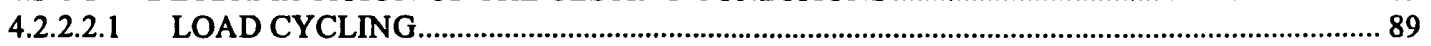

4.2.2.2.1.1 DETERMINATION OF THE NUMBER OF LOAD CYCLES ............................................. 89

4.2.2.2.1.2 DETERMINATION OF THE AMOUNT OF LOAD AND CYCLING RATE...................... 92

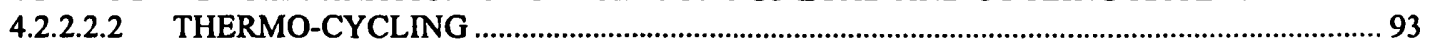

4.2.2.2.2.1 DETERMINATION OF THE THERMO-CYCLING CONDITIONS...................................93

4.2.2.3 SPECIMEN CONDITIONING ...........................................................................................94

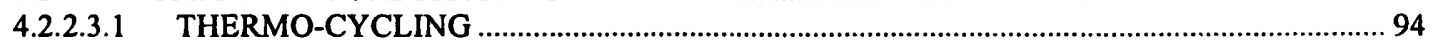

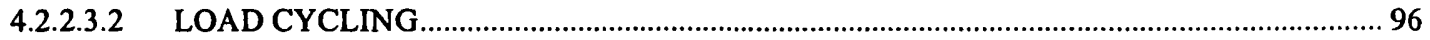

4.2.2.4 THREE POINT BEND TEST OF THE CONDITIONED SPECIMENS...................................... 96

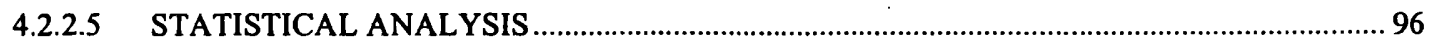

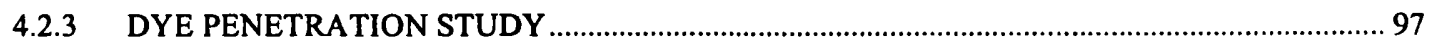

5. RESULTS

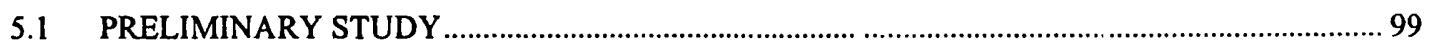

5.1.1 FLEXURAL TESTING TO DETERMINE THE MECHANICAL PROPERTIES OF THE

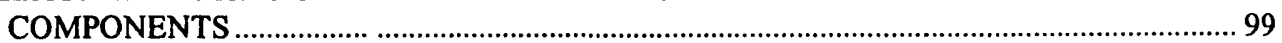

5.1.2 VARIATION IN THE MASS WEIGHING PROCEDURE ……………………….................... 100

5.1.3 WATER SORPTION AND SOLUBILITY OF THE RESIN COMPONENT …………………...... 101

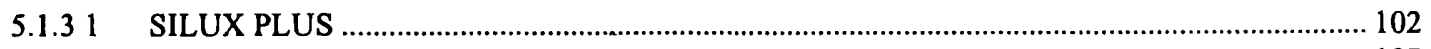

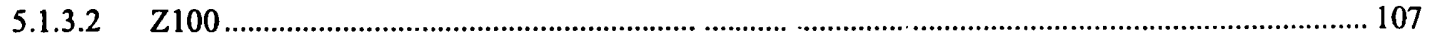

5.1.3.3 COMPARATIVE WATER SORPTION IN BETWEEN MATERIALS ...................................112

5.1.3.4 PH VALUES OF STORAGE WATER .............................................................................. 115 
5.2 MAIN LOAD CYCLING AND THERMO-CYCLING STUDY

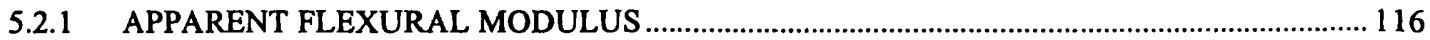

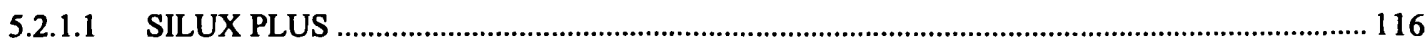

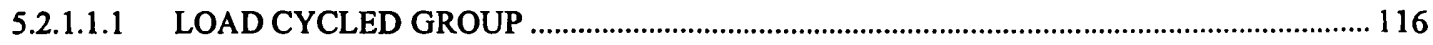

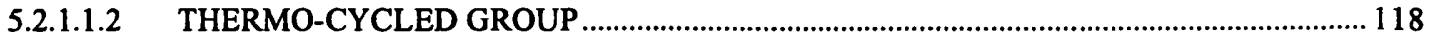

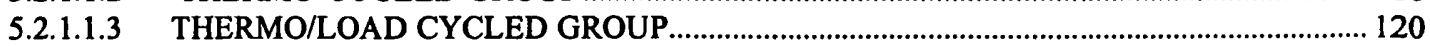

5.2.1.1.4 COMPARISON OF THE DIFFERENT SILUX PLUS CYCLING GROUPS ........................ 120

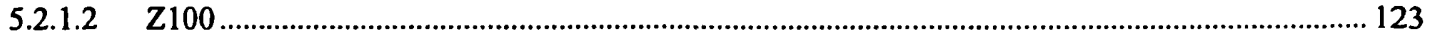

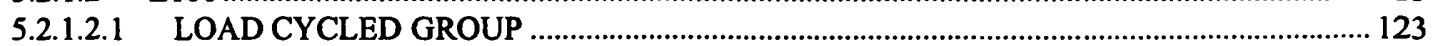

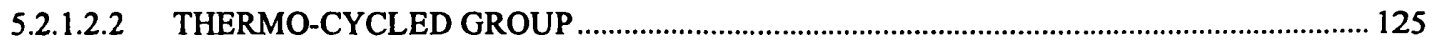

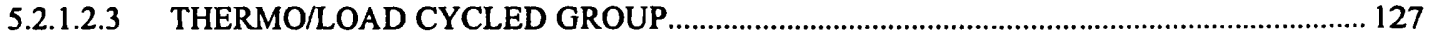

5.2.1.2.4 COMPARISON OF THE DIFFERENT Z100 CYCLING GROUPS.................................... 129

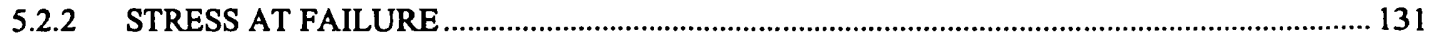

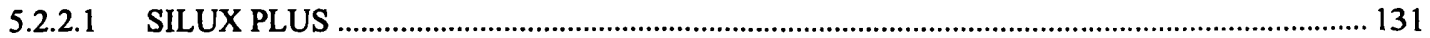

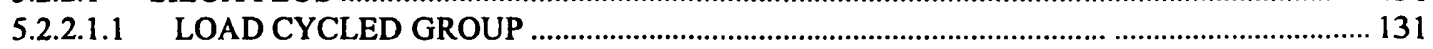

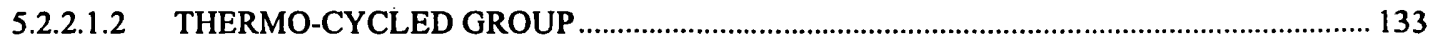

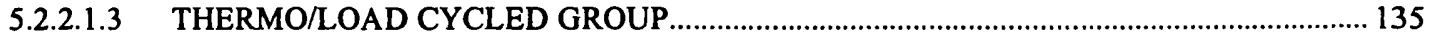

5.2.2.1.4 COMPARISON OF THE DIFFERENT SILUX PLUS CYCLING GROUPS .........................137

5.2.2.2 Z100

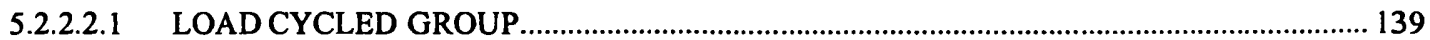

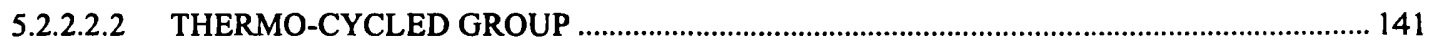

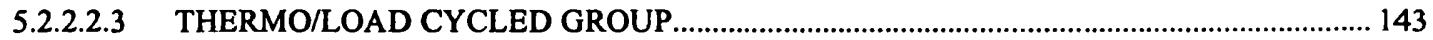

5.2.2.2.4 COMPARISON OF THE DIFFERENT Z100 CYCLING GROUPS..................................... 145

$5.2 .3 \quad$ EXTENSION AT FAILURE …................................................................................................ 147

5.2.4 STATISTICAL ANALYSIS TO VERIFY CHANGES WITHIN AND BETWEEN TESTING

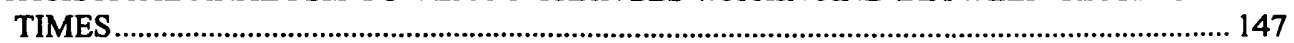

5.2.5 MODE OF FAILURE AND DYE PENETRATION TEST ….............................................. 151

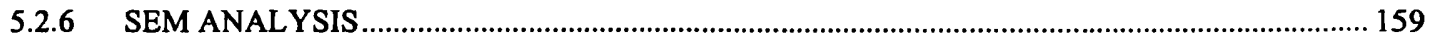

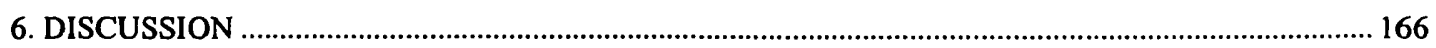

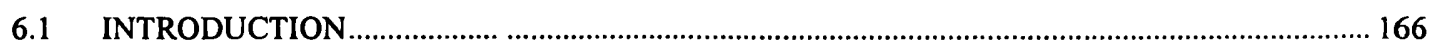

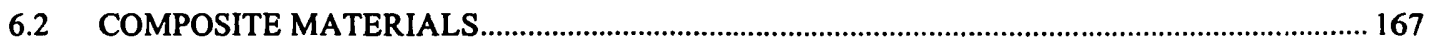

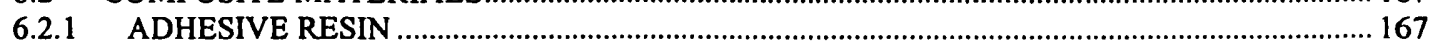

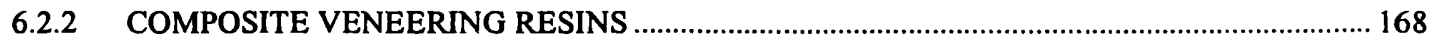

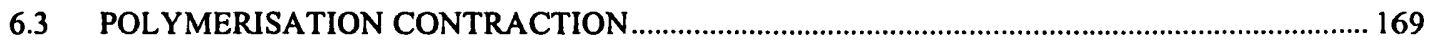

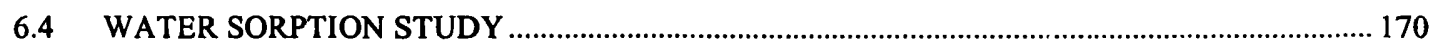

6.4.1 EFFECTS OF WATER SORPTION UPON THE SPECIMEN ................................................... 174

6.4.2 CONTROL OF EXPERIMENTAL VARIATION IN THE WATER SORPTION STUDY ........ 175

6.4.3 ANALYSIS OF THE EXPERIMENTAL FINDINGS OF THE WATER SORPTION

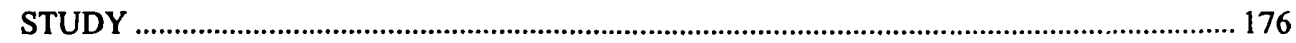

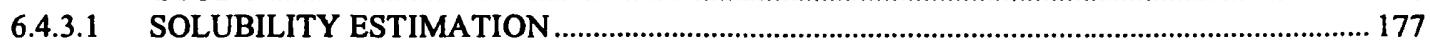

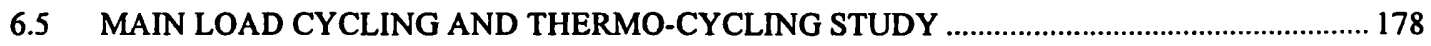

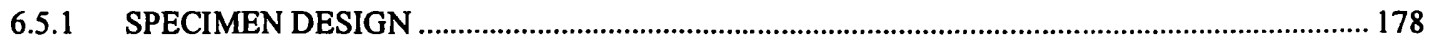

6.5.2 CONTROL OF EXPERIMENTAL VARIATION DURING SPECIMEN PREPARATION ....... 179

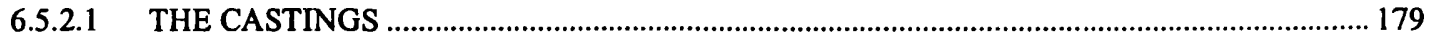

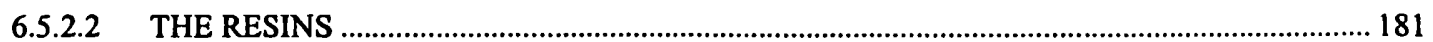

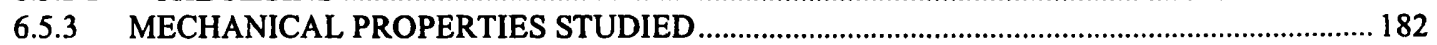

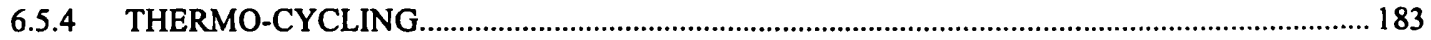

6.5.4.1 EFFECTS OF THERMO-CYCLING UPON THE SPECIMEN …...................................... 184

6.5.4.2 CONTROL OF EXPERIMENTAL VARIATION DURING THERMO-CYCLING ................ 185

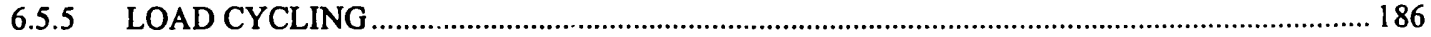

6.5.5.1 EFFECTS OF LOAD CYCLING UPON THE SPECIMEN …............................................... 189

6.5.5.2 CONTROL OF EXPERIMENTAL VARIATION DURING LOAD CYCLING ..................... 192

6.5.6 DISCUSSION OF THE EXPERIMENTAL FINDINGS OF THE MAIN LOAD CYCLING AND

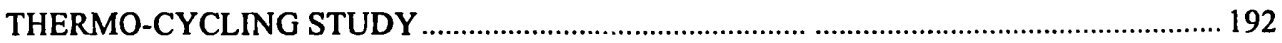

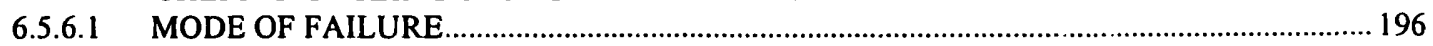

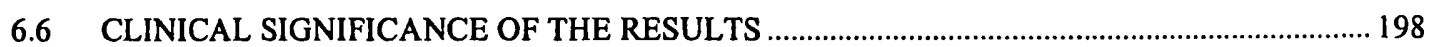


7. SUGGESTIONS FOR FURTHER RESEARCH .

.200

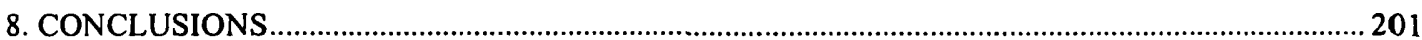

ACKNOWLEDGEMENTS

REFERENCES

APPENDIX I

APPENDIX II

APPENDIX III 


\section{LIST OF TABLES}

TABLE 2.1: COMPARATIVE VICKERS HARDNESS IN BETWEEN VARIOUS MATERIALS

TABLE 2.2 : COMPOSITION OF A TYPICAL CO/CR ALLOY (VITALLIUM) …………………... 9

TABLE 5.1 : CO/CR ALLOY AS PREPARED FOR LAMINATION (FLEXURAL TESTING) ... 99

TABLE 5.2 : $\quad$ SILUX PLUS RESIN SPINES (FLEXURAL TESTING) .............................................. 99

TABLE 5.3 : Z100 RESIN SPINES (FLEXURAL TESTING) ......................................................... 100

TABLE 5.4 : $\quad$ MASS OF METAL BLADE TO ESTABLISH VARIATION IN WEIGHING PROCEDURE ......................................................................................................... 101

TABLE 5.5 : $\quad$ SILUX PLUS WATER SORPTION $\left(1^{\text {ST }}\right.$ CYCLE MEAN \% VALUES $)$..................... 102

TABLE 5.6 : SILUX PLUS WATER SORPTION ( $2^{\text {ND }}$ CYCLE MEAN \% VALUES) .................... 104

TABLE 5.7: SOLUBILITY (SILUX PLUS MEAN \% VALUES).................................................. 106

TABLE 5.8 : Z100 WATER SORPTION ( ${ }^{\text {ST }}$ CYCLE MEAN \% VALUES).................................... 107

TABLE 5.9: $\quad$ Z100 WATER SORPTION (2 ${ }^{\text {ND }}$ CYCLE MEAN \% VALUES)............................... 110

TABLE 5.10 : SOLUBILITY (Z100 MEAN \% VALUES) ............................................................. 111

TABLE 5.11 : COMPARISON OF MEAN \% WATER SORPTION FOR SILUX PLUS AND Z100 IN BOTH SORPTION CYCLES ...................................................................................... 112

TABLE 5.12: MEAN POST TEST PH VALUES OF THE WATER IN WHICH THE SPECIMENS

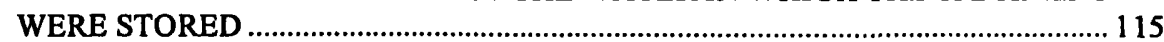

TABLE 5.13: APPARENT FLEXURAL MODULUS OF LOAD CYCLED SILUX PLUS …….....117

TABLE 5.14: STATISTICAL ANALYSIS OF APPARENT FLEXURAL MODULUS FOR LOAD

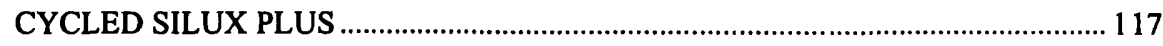

TABLE 5.15: $\quad$ APPARENT FLEXURAL MODULUS OF THERMO-CYCLED SILUX PLUS

TABLE 5.16: $\quad$ STATISTICAL ANALYSIS OF APPARENT FLEXURAL MODULUS FOR THERMO-CYCLED SILUX PLUS.

TABLE 5.17: APPARENT FLEXURAL MODULUS OF THERMO/LOAD CYCLED SILUX PLUS

TABLE 5.18 : STATISTICAL ANALYSIS OF APPARENT FLEXURAL MODULUS FOR THERMO/LOAD CYCLED SILUX PLUS.

TABLE 5.19: $\quad$ APPARENT FLEXURAL MODULUS OF LOAD CYCLED Z100 124

TABLE 5.20: STATISTICAL ANALYSIS OF APPARENT FLEXURAL MODULUS FOR LOAD

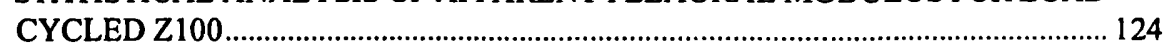

TABLE 5.21 : APPARENT FLEXURAL MODULUS OF THERMO-CYCLED Z100. 126

TABLE 5.22: $\quad$ STATISTICAL ANALYSIS OF APPARENT FLEXURAL MODULUS FOR THERMO-CYCLED Z100. 
TABLE 5.23 : $\quad$ APPARENT FLEXURAL MODULUS OF THERMO/LOAD CYCLED Z100 ....... 128

TABLE 5.24 : $\quad$ STATISTICAL ANALYSIS OF APPARENT FLEXURAL MODULUS FOR THERMO/LOAD CYCLED Z100

TABLE 5.25 : $\quad$ STATISTICAL ANALYSIS OF APPARENT FLEXURAL MODULUS FOR THE DIFFERENT Z100 TESTING GROUPS .................................................................. 129

TABLE 5.26 : $\quad$ STRESS AT FAILURE OF LOAD CYCLED SILUX PLUS .................................. 132

TABLE 5.27 : STATISTICAL ANALYSIS OF STRESS AT FAILURE FOR LOAD CYCLED SILUX PLUS

TABLE 5.28 : $\quad$ STRESS AT FAILURE OF THERMO-CYCLED SILUX PLUS. 134

TABLE 5.29 : $\quad$ STATISTICAL ANALYSIS OF STRESS AT FAILURE FOR THERMO-CYCLED SILUX PLUS

TABLE 5.30 : $\quad$ STRESS AT FAILURE OF THERMO/LOAD CYCLED SILUX PLUS 136

TABLE 5.31 : $\quad$ STATISTICAL ANALYSIS OF STRESS AT FAILURE FOR THERMO/LOAD CYCLED SILUX PLUS

TABLE 5.32: $\quad$ STATISTICAL ANALYSIS OF STRESS AT FAILURE FOR THE DIFFERENT SILUX PLUS TESTING GROUPS

TABLE 5.33 : $\quad$ STRESS AT FAILURE OF LOAD CYCLED Z100 …............................................ 140

TABLE 5.34 : $\quad$ STATISTICAL ANALYSIS OF STRESS AT FAILURE FOR LOAD CYCLED Z100

TABLE 5.35: $\quad$ STRESS AT FAILURE OF THERMO-CYCLED Z100.. 142

TABLE 5.36 : STATISTICAL ANALYSIS OF STRESS AT FAILURE FOR THERMO-CYCLED Z100

TABLE 5.37 : $\quad$ STRESS AT FAILURE OF THERMO/LOAD CYCLED Z100 144

TABLE 5.38 : STATISTICAL ANALYSIS OF STRESS AT FAILURE FOR THERMO/LOAD CYCLED Z100.

TABLE 5.39: STATISTICAL ANALYSIS OF STRESS AT FAILURE FOR THE DIFFERENT Z100 TESTING GROUPS.

TABLE 5.40 : STATISTICAL ANALYSIS OF APPARENT FLEXURAL MODULUS OF EACH WEEK SILUX PLUS POPULATION COMPARED TO THE REST OF POPULATIONS OF OTHER WEEKS

TABLE 5.41 : $\quad$ STATISTICAL ANALYSIS OF STRESS AT FAILURE OF EACH WEEK SILUX PLUS POPULATION COMPARED TO THE REST OF POPULATIONS OF OTHER WEEKS

TABLE 5.42: $\quad$ STATISTICAL ANALYSIS OF APPARENT FLEXURAL MODULUS OF EACH WEEK Z100 POPULATION COMPARED TO THE REST OF POPULATIONS OF EACH OTHER WEEK.

TABLE 5.43 : $\quad$ STATISTICAL ANALYSIS OF STRESS AT FAILURE OF EACH WEEK Z100 POPULATION COMPARED TO THE REST OF POPULATIONS OF OTHER WEEKS 
TABLE 5.44 : $\quad$ SILUX PLUS DYE PENETRATION MEASUREMENTS AND MODE OF FAILURE. 152

TABLE 5.45: Z100 DYE PENETRATION MEASUREMENTS AND MODE OF FAILURE ....... 153 TABLE 5.46 : SILUX PLUS MODE OF FAILURE ………............................................................... 154

TABLE 5.47 : Z100 MODE OF FAILURE................................................................................. 


\section{LIST OF FIGURES}

FIGURE 2.1 : SIMPLIFIED REPRESENTATION OF THE FORMATION OF A FREE RADICAL IN HEAT, COLD AND LIGHT CURED SYSTEMS.

FIGURE 2.2: SCHEMATIC ILLUSTRATION OF THE STRUCTURE OF A MICROFINE COMPOSITE CONTAINING PREPOLYMERISED PARTICLES.

FIGURE 2.3 : SCHEMATIC ILLUSTRATION OF THE STRUCTURE OF A HYBRID HEAVILY FILLED COMPOSITE

FIGURE 2.4: SIMPLIFIED SCHEMATIC REPRESENTATION OF SILANE BONDING BETWEEN THE FILLERS AND THE POLYMER MATRIX IN DENTAL COMPOSITES

FIGURE 2.5 : $\quad$ SIMPLIFIED SCHEMATIC REPRESENTATION OF THE 10METHACRYLOYLOXY-DECYL-DIHYDROGEN PHOSPHATE (10-MDP) LINK TO THE METAL SURFACE

FIGURE 4.1 : THE KULZER UNILUX AC LIGHT CURING VESSEL 69

FIGURE 4.2 : DIMENSIONS OF THE JIG USED TO TEST THE COMPOSITE RESIN SPINE SPECIMENS 70

FIGURE 4.3A : THE SPECIMEN MOULD FOR THE WATER SORPTION STUDY... 72

FIGURE 4.3B : THE DIMENSIONS OF THE SPECIMEN MOULD FOR THE WATER SORPTION STUDY

FIGURE 4.4A : A TYPICAL SPECIMEN FOR THE WATER SORPTION STUDY .. 73

FIGURE 4.4B : THE DIMENSIONS OF A TYPICAL SPECIMEN FOR THE WATER SORPTION STUDY

FIGURE 4.5 : DIAGRAM OF THE JG USED TO ABRADE THE METAL ALLOY SPECIMENS

FIGURE 4.6 : FLOW DIAGRAM OF THE PROCEDURES UNDERTAKEN TO MANUFACTURE THE RESIN BONDED TO METAL LAMINATES.

FIGURE 4.7A : THE SPECIMEN MOULD USED TO MANUFACTURE THE RESIN TO METAL BONDED SPECIMENS.

FIGURE 4.7B : A REPRESENTATION OF THE DIFFERENT LENGTHS OF THE VARIOUS COMPONENTS OF THE SPECIMEN MOULD USED TO FABRICATE THE BONDED RESIN TO METAL SPECIMENS

FIGURE 4.8A : A CROSS-SECTIONAL VIEW OF THE SPECIMEN MOULD USED TO FABRICATE THE BONDED RESIN TO METAL SPECIMENS.

FIGURE 4.8B : A CROSS-SECTIONAL REPRESENTATION OF THE DIFFERENT LAYERS OF THE SPECIMEN MOULD USED TO FABRICATE THE BONDED RESIN TO METAL SPECIMENS.

FIGURE 4.9A : A TYPICAL SPECIMEN 83

FIGURE 4.9B : DIMENSIONS OF A TYPICAL BONDED SPECIMEN 83

FIGURE 4.10: LAYERS OF MATERIALS USED FOR THE MANUFACTURE OF SPECIMENS 
FIGURE 4.11 : HANDLING OF SPECIMENS AFTER MANUFACTURE .......................................... 85

FIGURE 4.12 : WATER CONDITIONED SPECIMEN HANDLING ………………………................... 86

FIGURE 4.13A ： A MOUNTED SPECIMEN ON THE TESTING JIG ………………………………....90

FIGURE 4.13B : $\quad$ AREA OF THE SPECIMEN WHERE LOAD IS APPLIED ...........................................90

FIGURE 4.14A ： THE TESTING JIG.............................................................................................. 91

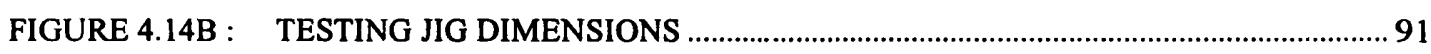

FIGURE 4.15A： THE THERMO-CYCLING MACHINE ………………………………………....... 95

FIGURE 4.15B : THE DIFFERENT WATER BATHS OF THE THERMO-CYCLING MACHINE..... 95

FIGURE 5.1 : SILUX PLUS: SCATTER DISTRIBUTION OF INDIVIDUAL SPECIMENS MASS

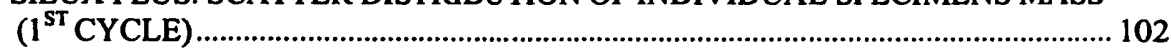

FIGURE 5.2: $\quad$ SILUX PLUS: MEAN WATER SORPTION ( ${ }^{\text {ST }}$ CYCLE) ....................................... 103

FIGURE 5.3 : $\quad$ SILUX PLUS: INCREASE IN MASS WITH RESPECT TO $\mathrm{T}^{1 / 2}\left(1^{\mathrm{ST}}\right.$ CYCLE) ........ 103

FIGURE 5.4 : $\quad$ SILUX PLUS: SCATTER DISTRIBUTION OF INDIVIDUAL SPECIMENS MASS

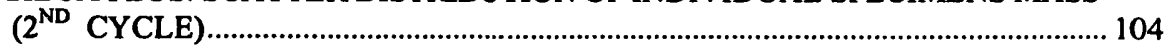

FIGURE 5.5 : $\quad$ SILUX PLUS: MEAN WATER SORPTION (2 ${ }^{\text {ND }}$ CYCLE) ..................................... 105

FIGURE 5.6 : SILUX PLUS: INCREASE IN MASS WITH RESPECT TO T ${ }^{1 / 2}\left(2^{\mathrm{ND}}\right.$ CYCLE) ........ 105

FIGURE 5.7 : Z100: SCATTER DISTRIBUTION OF INDIVIDUAL SPECIMENS MASS

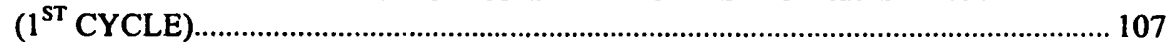

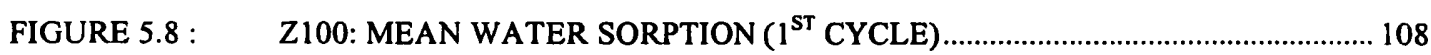

FIGURE 5.9 : Z100: INCREASE IN MASS WITH RESPECT TO $\mathrm{T}^{1 / 2}\left(1^{\mathrm{ST}}\right.$ CYCLE) ........................ 109

FIGURE 5.10: Z100: SCATTER DISTRIBUTION OF INDIVIDUAL SPECIMENS MASS

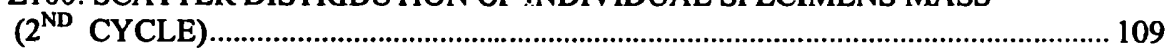

FIGURE 5.11： Z100: MEAN WATER SORPTION (2 $2^{\text {ND }}$ CYCLE) ..............................................

FIGURE 5.12: Z100: INCREASE IN MASS WITH RESPECT TO $\mathrm{T}^{1 / 2}\left(2^{\mathrm{ND}}\right.$ CYCLE) .....................111

FIGURE 5.13 : COMPARATIVE \% WATER SORPTION FOR SILUX PLUS AND Z100

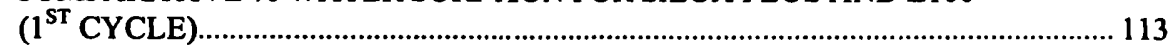

FIGURE 5.14 : COMPARATIVE \% WATER SORPTION FOR SILUX PLUS AND Z100

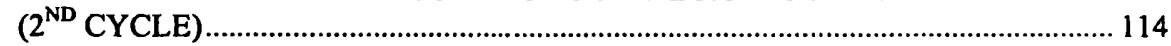

FIGURE 5.15 : APPARENT FLEXURAL MODULUS OF LOAD CYCLED SILUX PLUS............. 117

FIGURE 5.16: APPARENT FLEXURAL MODULUS OF THERMO-CYCLED SILUX PLUS...... 119

FIGURE 5.17: APPARENT FLEXURAL MODULUS OF THERMO/LOAD CYCLED SILUX PLUS.

FIGURE 5.18: $\quad$ APPARENT FLEXURAL MODULUS FOR THE DIFFERENT SILUX PLUS TESTING GROUPS .................................................................................................. 122

FIGURE 5.19 : APPARENT FLEXURAL MODULUS OF LOAD CYCLED Z100 …………………... 124 
FIGURE 5.20: $\quad$ APPARENT FLEXURAL MODULUS OF THERMO-CYCLED Z100 126

FIGURE 5.21 : APPARENT FLEXURAL MODULUS OF THERMO/LOAD CYCLED Z100........ 128

FIGURE 5.22: $\quad$ APPARENT FLEXURAL MODULUS FOR THE DIFFERENT Z100 TESTING

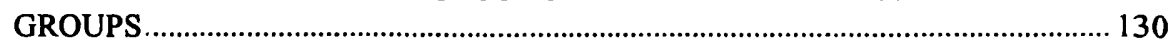

FIGURE 5.23 : $\quad$ STRESS AT FAILURE OF LOAD CYCLED SILUX PLUS …............................... 132

FIGURE 5.24 : $\quad$ STRESS AT FAILURE OF THERMO-CYCLED SILUX PLUS ............................. 134

FIGURE 5.25 : $\quad$ STRESS AT FAILURE OF THERMO/LOAD CYCLED SILUX PLUS ................... 136

FIGURE 5.26 : STRESS AT FAILURE FOR THE DIFFERENT SILUX PLUS TESTING

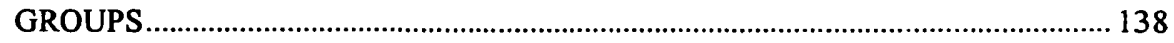

FIGURE 5.27 : $\quad$ STRESS AT FAILURE OF LOAD CYCLED Z100 ............................................... 140

FIGURE 5.28 : $\quad$ STRESS AT FAILURE OF THERMO-CYCLED Z100 ............................................ 142

FIGURE 5.29 : $\quad$ STRESS AT FAILURE OF THERMO/LOAD CYCLED Z100................................ 144

FIGURE 5.30 : $\quad$ STRESS AT FAILURE FOR THE DIFFERENT Z100 TESTING GROUPS............ 146

FIGURE 5.31 : $\quad$ EXTENSION AT FAILURE FOR THE DIFFERENT TESTING GROUPS............. 148

FIGURE 5.32A : A COHESIVE FAILURE WITH THE FRACTURE LINE DIVIDED ..................... 156

FIGURE 5.32B : A COHESIVE FAILURE WITH THE FRACTURE LINE DIVIDED ..................... 156

FIGURE 5.33A : $\quad$ A COHESIVE FAILURE WITH A SINGLE FRACTURE LINE............................. 157

FIGURE 5.33B : A COHESIVE FAILURE WITH A SINGLE FRACTURE LINE ............................ 157

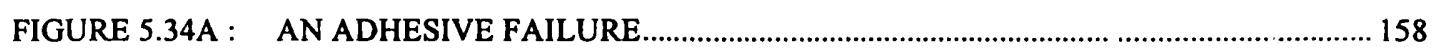

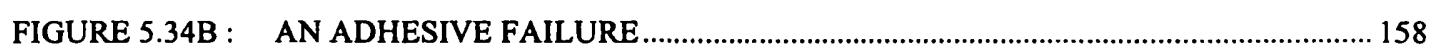

FIGURE 5.35A : $\quad$ SILUX PLUS 2 WEEKS THERMO-CYCLED GROUP (MICROGRAPH)........... 160

FIGURE 5.35B : SILUX PLUS 2 WEEKS THERMO-CYCLED GROUP (MICROGRAPH) .............. 160

FIGURE 5.36A : SILUX PLUS 2 WEEKS THERMO/LOAD CYCLED GROUP (MICROGRAPH) 161

FIGURE 5.36B : SILUX PLUS 2 WEEKS THERMO/LOAD CYCLED GROUP (MICROGRAPH). 161

FIGURE 5.37A : $\quad$ SILUX PLUS 20 WEEKS THERMO-CYCLED GROUP (MICROGRAPH)........... 162

FIGURE 5.37B : SILUX PLUS 20 WEEKS THERMO-CYCLED GROUP (MICROGRAPH) ........... 162

FIGURE 5.38A : $\quad$ Z100 2 WEEKS THERMO-CYCLED GROUP (MICROGRAPH) ........................... 163

FIGURE 5.38B : $\quad$ Z100 2 WEEKS THERMO-CYCLED GROUP (MICROGRAPH).......................... 163

FIGURE 5.39A : Z Z100 2 WEEKS THERMO/LOAD CYCLED GROUP (MICROGRAPH) ............... 164

FIGURE 5.39B : Z100 2 WEEKS THERMO/LOAD CYCLED GROUP (MICROGRAPH) ................ 164

FIGURE 5.40A : Z100 20 WEEKS THERMO/LOAD CYCLED GROUP (MICROGRAPH)............. 165

FIGURE 5.40B : Z100 20 WEEKS THERMO/LOAD CYCLED GROUP (MICROGRAPH) .............. 165 
FIGURE 6.1: $\quad$ REPRESENTATION OF THE STRESSES SET-UP AT THE INTERFACES OF THE LAMINATE WHEN A LOAD IS APPLIED . 


\section{GENERAL INTRODUCTION}

\subsection{THE NEED FOR OVERLAY REMOVABLE PARTIAL DENTURES (RPDS)}

Watson (1986), defined the overlay denture as "a removable appliance which covers the occlusal surfaces of the teeth and alters the contact with the natural teeth, often by increasing the vertical dimension". He suggested several reasons for providing an overlay denture. These are:

a) to achieve a stable, satisfactory horizontal and vertical relationship of the maxilla and mandible,

b) to prevent damage to the oral tissues arising from malocclusion,

c) to improve the appearance by altering the facial proportions or by modifying irregular dental arches,

d) to ameliorate symptoms of temporomandibular joint dysfunction, including those which have arisen from pathological changes in these structures.

Three groups of patients, in particular, frequently require provision of overlay dentures, those with hypodontia, malformed teeth or those with advanced tooth wear. The objective of treatment is to restore form and function of the missing tissues. The materials employed for the construction of an overlay denture should try to reproduce the properties of the tissues they replace. They should not interfere with normal oral function and not cause a toxic or allergic reaction. It is desirable that they should also be able to withstand the adverse conditions of the oral environment. Overlay dentures can be either acrylic resin or metal alloy based:

a) Acrylic resin based dentures are usually prescribed as provisional prostheses, to assess changes in the occlusal vertical dimension or appearance.

b) Metal alloy dentures veneered with polymer resin are generally needed for definitive treatment. 


\subsection{FAILURE MODES IN METAL BASED OVERLAY REMOVABLE PARTIAL DENTURES (RPDs)}

Hobkirk et al (1989) assessed the failure rates and modes of failure of overlay dentures used in the treatment of severe hypodontia. They found high failure rates in some components. They further reported that $30 \%$ of the acrylic resin onlays had been replaced after six to thirty months due to wear and $8 \%$ due to fracture. $16 \%$ of anterior teeth without backings broke off the dentures, many within the first year. Previously, Braem et al (1986a) when they examined the deterioration of posterior composite restorations suggested that these two failure modes are often related to areas of occlusal contact where wear is often accompanied by crack formation.

Wear of the veneering material and any adhesive or cohesive failure of the components of the RPD are crucial when considering long term economics, function and aesthetics of the prosthesis. Such failures have not only financial implications but may cause diminished chewing performance and changes in occlusion.

\subsection{PREDISPOSING FACTORS PRECIPITATING OVERLAY FAILURES}

These can either be related to the patient, the design of the denture or to the limitations of the materials being employed.

Patient related factors could be:

a) Biting forces, exhibited during normal functional or parafunctional activity.

b) External trauma, or the accidental damage of the prosthesis by the patient.

There are numerous reports in the dental literature that describe the measurement of biting forces on teeth. Because of their dynamic nature, the actual biting stresses during mastication are difficult to measure and test conditions are difficult to standardise 
(Fløystrand et al 1982, Boretti et al 1995, Paphangkorakit and Osborn 1998). Therefore the figures reported vary enormously. Also, the biting force varies markedly from one area of the mouth to another and from one individual to another (Tortopidis et al 1998). The maximal force also varies with the state of the dentition, dentate subjects being capable of achieving higher biting forces than edentulous subjects (Hellsing 1980).

In the natural dentition, the forces recorded in the incisal region vary from 13.2 to $23.1 \mathrm{~kg}$ (129 to $227 \mathrm{~N}$ ) (Bates et al 1975), while Clark and Carter (1985) though found that the maximum biting force in the molar region to be $36.1 \mathrm{~kg}(354 \mathrm{~N})$. In the light of these findings, it is always wise to overengineer the partial denture if there are symptoms or signs of parafunctional activity.

The design of the various components of a partial denture should follow the generally accepted prosthetic principles of retention, stability and support. Failure to adhere to these principles may cause failure of the prosthesis very often in the very short term. Failure of the partial denture may also be caused by poor, or inappropriate use of materials. Many of these failures are the result of the effects of fatigue (McCabe et al 1990, Braem et al $1994 a+b)$. Long term degradation due to water uptake and solubility will also lead to failure (Gale and Darvell 1999). It is particularly these failures concerned with combined environmental-mechanical behaviour which are of significance (Anusavice and de Rijk 1990). The properties of the available materials may not be suitable for long term use and this can lead to failure of the denture.

The severe conditions of the oral environment, make the correlation between in vitro data and clinical performance of a material very difficult (Braem et al 1994a+b). An analysis of the failure potential is often represented by measurement of (1) elastic or reversible 
deformation, that is, proportional limit, resilience, modulus of elasticity, (2) plastic or irreversible deformation, for example, percentage of elongation or (3) a combination of elastic and plastic deformation, such as toughness and failure strength. It is not uncommon that modulus of elasticity data is often used to characterise fatigue sensitive resin composites and their resistance to deformation. The modulus is relatively easy to determine, provides a convenient characterisation of an unknown material and is often used to explain the behaviour of resin composites under occlusal stress (Braem et al $1994 a+b)$.

An analysis of the failure potential of an overlay partial denture under applied forces, must be related to the mechanical properties of the combination of the constituent prosthetic materials. Trying to predict in vivo prosthesis behaviour with in vitro testing is a challenging task. Not only do testing conditions have to be carefully selected, but also the testing of specimens should be made with the same combination of constituent materials in a similar manner to the way they are employed intra-orally. 


\section{LITERATURE REVIEW}

\subsection{MATERIALS USED FOR THE MANUFACTURE OF OVERLAY REMOVABLE PARTIAL DENTURES (RPDs)}

The clinician designing an overlay RPD, tries to provide a prosthesis by replacing lost tissues in order to restore function and aesthetics for the patient. A successful design, should not only address the requirements of retention, support and stability for the prosthesis, but also replace any missing tissues with materials that have similar aesthetic characteristics. This could necessitate the covering of metal clasp arms or occlusal rests with resin polymers that look like the soft tissue or tooth.

While the design of an overlay RPD should be undertaken on a surveyed master model for the various aspects of the arch to be thoroughly examined, an estimation of the nature and value of the forces that the restoration would be subjected to is also of prime importance. This estimation together with the design findings would help determine the material used for various component parts like major and minor connectors, retentive clasps, flanges and artificial teeth. Ability to withstand, high cyclic load and thermal stresses without fracture, chemical and environmental degradation, abrasive forces without undergoing wear and without causing wear to antagonistic surfaces, could determine the type of material employed.

\subsubsection{CHOICE OF THE METAL ALLOY COMPONENT}

Some of the factors that may influence the choice of alloy, include the availability of space for the major connector, the amount and location of the undercut area on the proposed abutments and the complexity of the framework design. Further, the question of whether precision attachments are to be used and the availability of appropriate technical support and financial resources need to be addressed. The metal alloy component, can act 
as a framework and provide the rigidity needed to resist and distribute functional forces around the supporting teeth or act as a retentive element to help retain the prosthesis in function.

Currently, base metal alloys have largely replaced gold alloys in the fabrication of RPD frameworks (Lammie and Laird 1986). Since 1969, when the United States Government lifted its controls on the price of gold, allowing supply and demand to determine its value, the price has increased, causing a substantial rise in the price of all dental gold alloys. Although alloys containing higher percentages of noble metals are preferred for crown and bridge prostheses, many alloys containing a lower percentage of gold or even base metal alloys are used, because of their lower cost. Recently though, a significant drop in the price of gold has occurred. This might affect the choice of the metal alloy for prosthetic applications, in the immediate future. The type IV, or extra hard gold alloys are designed to have sufficient strength and adequate properties to be employed as cast RPDs.

Although, as previously explained, the popularity of the base metal alloys stems mainly from their lower cost, some of their physical properties also offer advantages in particular clinical conditions. Perhaps the most significant difference in the properties of the two alloys is the modulus of elasticity, with the base metal alloys possessing values much higher than gold alloys. Where there is limited space for major connectors and other components that require rigidity, the base metal alloys offer distinct advantages. The base metal alloys have significantly higher proportional limits or failure strengths when compared to the more ductile gold alloys. When flexibility is desirable, as in the retentive portion of a clasp, the gold alloys are superior. Gold alloys exhibit elongation values higher than base metals and generally are much softer allowing for easy polishing and burnishing. 
Another property to be considered is the effect of the restorative material wearing the opposing dentition. Studies have shown that gold alloys will cause little wear to opposing enamel indicating their suitability as occlusal surfaces, specially when parafunctional habits are present (Mahalick et al 1971, Jagger and Harrison 1995, Koczorowski and Wloch 1999). Different values of Vickers hardness for different materials compared to enamel and dentine can be seen in Table 2.1 .

Various non-noble metal alloys such as cobalt-chromium $(\mathrm{Co} / \mathrm{Cr})$, or nickel-chromium (Ni/Cr), have been employed for fabricating RPD frameworks, replacing gold alloys almost completely. Nickel, though, is one of the most common allergens and the most potent sensitiser of all the metals (Covington et al 1985, Jones et al 1986). Beryllium, a component of many alloys, would appear to be more hazardous to the dental technician as a potential carcinogen (Moffa et al 1973). As a result, beryllium and nickel free $\mathrm{Co} / \mathrm{Cr}$ alloys are the most commonly used based metal alloys for RPD construction. The composition of a typical $\mathrm{Co} / \mathrm{Cr}$ alloy is shown in Table 2.2 .

The fusion temperatures of the $\mathrm{Ni} / \mathrm{Cr}$ and $\mathrm{Co} / \mathrm{Cr}$ alloys vary between $1200-1500^{\circ} \mathrm{C}$ whereas those for casting gold alloys rarely have fusion temperatures above $950^{\circ} \mathrm{C}$. Melting of gold alloys can readily be achieved using a gas-air mixture whereas base metal alloys however require an acetylene-oxygen flame or an electrical induction furnace. The latter method is favoured since it operates under more controlled conditions.

When using oxyacetylene flames the ratio of oxygen to acetylene must be carefully controlled. Excess oxygen may cause oxidation of the alloy whilst an excess of acetylene produces an increase in the metal carbide content leading to embrittlement. 


\begin{tabular}{|c|c|}
\hline Material & $\begin{array}{c}\text { Vickers } \\
\text { Hardness } \\
\text { (kg/mm })^{2}\end{array}$ \\
\hline Heat cured PMMA & 23 \\
\hline $\begin{array}{c}\text { Microfine composite } \\
\text { (Silux Plus) }\end{array}$ & 59 \\
\hline $\begin{array}{c}\text { Hybrid composite } \\
\text { (Z100) }\end{array}$ & 120 \\
\hline Ni/Cr alloy & $270-395$ \\
\hline Co/Cr alloy & $350-390$ \\
\hline Type IV gold alloy & $160-250$ \\
\hline Feldspathic porcelain & 703 \\
\hline Dentine & $57-60$ \\
\hline Enamel & $294-408$ \\
\hline
\end{tabular}

Table 2.1 : Comparative Vickers hardness in between various materials $\left(\mathrm{kg} / \mathrm{mm}^{2}\right)$ (O'Brien 1997) 


\begin{tabular}{|c|c|}
\hline Element & $\%$ of reight \\
\hline Chromium & 30.0 \\
\hline Cobalt & Balance \\
\hline Molybdenum & 5.0 \\
\hline Iron & 1.0 \\
\hline Carbon & 0.5 \\
\hline Silicon & 0.6 \\
\hline Manganese & 0.5 \\
\hline
\end{tabular}

Table 2.2 : Composition of a typical $\mathrm{Co} / \mathrm{Cr}$ alloy (Vitallium) (Craig 1997a)

$\mathrm{Co} / \mathrm{Cr}$ alloy has high failure and tensile strengths and a high modulus of elasticity (Craig 1997b). This makes $\mathrm{Co} / \mathrm{Cr}$ alloy frameworks rigid in thin cross-sections, and able to withstand high occlusal forces. The hardness is about one third greater than Type IV gold alloys (Table 2.1). Its rigidity, precludes its manipulation with pliers due to excessive work hardening. Its use in areas of flexure as in the clasps of an RPD is limited. Osborne and Lammie (1953) stressed the importance of the clasp thickness and Bates (1963), suggested that a $\mathrm{Co} / \mathrm{Cr}$ clasp arm, bending through $180^{\circ}$, should be at least $15 \mathrm{~mm}$ long and engage an undercut of $0.25 \mathrm{~mm}$. 
In the "as cast" state, the alloy surface is normally quite rough, partially due to the coarse nature of the investing material. Base metal alloys and particularly the $\mathrm{Co} / \mathrm{Cr}$ type, are very hard and consequently difficult to polish. After casting it is usual to sandblast the metal to remove any surface roughness or adherent investment material as well as the green layer of oxide which coats the surface after casting. Final polishing can be carried out using a high speed polishing buffing mop.

\subsubsection{CHOICE OF THE VENEERING COMPONENT}

Wherever there are aesthetic demands necessitating the use of a tooth or soft tissue coloured material, porcelain, acrylic resin, or composite resin can be used.

\subsubsection{CERAMICS}

Feldspathic porcelain has been traditionally used as an aesthetic replacement of missing tooth substance. It is inherently brittle and must be used clinically in such a way as to minimise the effect of this property. Although traditional porcelain has a relatively high compressive strength (345 MPa), it possesses a low transverse strength (55-62 $\mathrm{MPa}$ ). Porcelain teeth for example should not be subjected to large tensile stresses to avoid catastrophic failure. Furthermore, the impact strength of this type of restorative is rather low.

To avoid fracture of porcelain under heavy occlusal forces, techniques that permitted veneering of porcelain to metallic substrates were introduced to enhance its strength, by fusing on to the surface of an appropriate alloy. Porcelain bonds to the surface of castings by mechanical and chemical interactions. Metallographic examination of the porcelainmetal interface reveals obvious mechanical interlocking in between the two surfaces. Further, tin and indium oxides are formed along grain boundaries into the underlying 
gold-based alloy, providing a mechanism that might explain the good adhesion seen with porcelain. These metal oxides are thought to dissolve into the glass of the opaque porcelain layer used to mask the metal substructure, and to create a strong chemical/micromechanical bond between the alloy and the ceramic (Peregrina et al 1992). The great advantage of porcelain occlusal surfaces is that they can maintain the designed occlusal scheme satisfactorily over the years and do not need regular refurbishments. Also, an excellent aesthetic result can be achieved that is stable over time. A precious metal alloy framework is however needed, in order for porcelain to be baked satisfactory.

Currently, the use of porcelain is limitedy for removable overlay prostheses. The time of treatment is prolonged and technical expertise is needed to reconstruct the occlusal surfaces. There is also considerable risk of abrasion to the opposing dentition, since ceramic in unglazed form is very rough. Any unglazed adjustments will cause considerable tooth tissue loss (Jagger and Harrison 1994).

In an attempt to reduce this problem, low fusing porcelains $\left(870^{\circ}-1065^{\circ}\right)$ have been developed. Apart from the low fusing products currently available, other all ceramic products have also been developed that do not need to be fused to a metal substructure for strength. These systems are widely used in fixed prosthodontics due to their superior aesthetic result. Although some all-ceramic products are less abrasive to antagonistic enamel compared to traditional feldspathic porcelain (Hacker et al 1996), others are still very abrasive to the antagonistic tooth (Rosenblum and Schulman 1997). There is also some difficulty in constructing the occlusal surface to conform with the antagonist teeth. 


\subsubsection{POLYMER VENEERING MATERIALS}

\subsection{ACRYLIC RESIN}

Acrylic resin is often used for the manufacture of artificial teeth or the reconstruction of soft tissues on an RPD due to its simplicity of use, ease of repair, relative low cost and good aesthetics.

Poly(methyl methacrylate) (PMMA) was developed by Imperial Chemical Industries (ICI) in 1931. Much of the early research work on the material was based around the construction of complete dentures. In 1934 ICI introduced Kallodent, the first injection moulded PMMA for dental application. Kulzer in 1938 patented the dough flask and pack technique which is still in use today for the manufacture of acrylic resin dentures. This involves the manipulation of a dough made from a PMMA/MMA powder/ liquid monomer system. The material is made by the process of free radical addition polymerisation of MMA to form PMMA with the use of a benzoyl peroxide catalyst and a tributyl amine as the activator.

PMMA is less rigid and brittle than porcelain and has much lower compressive and tensile strength/ On the other hand, it will not wear the opposing natural dentition due to its low hardness number (Table 2.1). Ease of fabrication, smooth surface texture, excellent aesthetics and quite acceptable mechanical properties including a relatively low modulus of elasticity, allowing for high displacement at failure during flexural deformation, are considered to be its main advantages. The main disadvantages of PMMA stem from its high polymerisation shrinkage and water sorption. As a veneering resin covering $\mathrm{Co} / \mathrm{Cr}$, its water uptake together with its low hardness, necessitate its frequent refurbishment due to occlusal wear, discolouration and marginal fracture in thin sections. Small defects and surface flaws caused by inadequate polymerisation or processing errors 
can weaken the acrylic resin structure especially when tensile stresses are encountered in the area of these flaws. Fracture toughness is a mechanical property that describes the resistance of materials to crack propagation under an applied stress. PMMA has low fracture toughness and if prostheses of complex geometry are manufactured, surface flaws or defects become more common and may lead to crack initiation.

In order to reinforce the denture base polymers, traditionally metal strengtheners were used in the form of wires or plates (Carroll and von Fraunhofer 1984, Ruffino 1985, Vallittu 1995). Nevertheless, the influence of wire reinforcement on the fatigue resistance of the polymers was minor (Vallittu 1996b). However, when metal strengtheners were surface treated by silicoating or sandblasting, the fracture resistance of the polymer/metal construction increased (Vallittu 1993b).

The reinforcement of PMMA denture bases to impact forces has been approached by the incorporation of a rubber phase. An interpenetrating network of styrene-butadiene and PMMA increases fracture resistance but its high cost limits the use to selected cases (Rodford 1986). It is relatively simple to increase the impact strength by rubber reinforcement but this does affect the elastic modulus. The higher the content of the low molecular weight polybutadiene based polymers, the higher the impact strength but the lower the elastic modulus (Rodford 1986). In the case of denture base materials, however, the latter must be maintained otherwise the appliance will be too flexible. The essential problem is to achieve a satisfactory compromise between impact strength and modulus.

To improve the strength and fatigue resistance of denture base polymers, reinforcing fibres were also used. Carbon fibres have proven clinically successful, (Schreiber 1971, Manley et al 1979, Bowman and Manley 1984, DeBoer et al 1984, Yazdanie and Mahood 
1985, Ekstrand et al 1987) except for reduced aesthetics. There was also a danger of them protruding from the denture.

Glass fibres on the other hand, had good aesthetic properties and were used in some studies to improve the mechanical properties of polymer materials (Vallittu 1993a, Vallittu et al 1994a, Vallittu et al 1994b, Vallittu et al 1995a, Vallittu and Narva 1997, Uzun et al 1999, Kanie et al 2000). Ideally, to strengthen polymer resins by including glass fibres, there should be good adhesion between the polymer matrix and the fibres. Untreated fibres act as inclusion bodies in the acrylic resin mixture and instead of strengthening, may actually weaken the resin (Jagger et al 1999). Treating the fibres with a silane compound can markedly increase the fracture resistance (Vallittu 1993a, Kanie et al 2000). Nevertheless, adequate impregnation of the reinforcing fibres with the resin has been difficult to achieve in all cases (Vallittu 1996a, Vallittu et al 1998). This led to increase in water sorption (Vallittu et al 1998), discolouration of the reinforcement due to the voids present, and decrease in the polymerisation within the composite due to entrapped oxygen (Miettinen and Vallittu 1997).

Ultra high molecular weight polyethylene fibres (UHMPE) were also tried, but showed mixed results (Gutteridge 1988, Gutteridge 1992, Ladizesky et al 1990, Dixon and Breeding 1992, Ladizesky et al 1992, Ladizesky et al 1993, Vallittu 1996a, Uzun et al 1999). One of the main problems was that these fibres did not bond well to the matrix (Takagi et al 1996). Ladizesky and Chow (1992) examined the mechanical properties of resins reinforced with continuous UHMPE fibres which were treated with plasma etching for enhanced adhesion. They demonstrated substantial improvements which were unaffected by water immersion and the presence of anatomical notches. 
Carlos and Harrison (1996) addressed the significance of the fibre orientation for them of being of benefit, i.e. running parallel to the surface of the denture. To date, the reinforcement of acrylic resin with UHMPE in the form of woven mat is a satisfactory way to improve the mechanical properties of acrylic resin. However, the technique is time consuming, prohibiting routine reinforcement of dentures (Jagger et al 1999).

Recently, a fibre reinforcement system (Stick ${ }^{*}$ and Stick-Net ${ }^{*}$ ), based on preimpregnation of reinforcing fibres with highly porous polymer has been introduced. Stick is made from continuous unidirectional glass fibres and Stick-Net is made from a woven glass fibre. They both allow good impregnation of fibres with polymer matrix, since the fibres are wetted with a mixture of polymer powder and monomer liquid or with monomers of light curing resin. Subsequently, the porous preimpregnation polymer can plasticise by dissolution.

Stick was found to increase the transverse strength and flexural modulus of both cold and heat cured denture base polymers (Vallittu 1999a). In the same study Stick-Net was found to increase the strain at fracture. These findings are very important from the clinical perspective since the reinforced polymers are stronger in thin sections. Apart from reinforcing denture bases, Stick has also been used as a fixed bridge framework (Vallittu 1999b). Nohrström et al (2000) found that the effectiveness of the reinforcement was more evident in long span bridges and that the orientation of the fibres influenced the final strength.

The reinforcement of PMMA has also been approached by self-reinforcement. Gilbert et al (1995) described the reinforcement of PMMA with chemically identical continuous

\footnotetext{
'Stick Tech Ltd., Turku, Finland
} 
PMMA fibres that were previously processed to impart large amounts of molecular orientation. That is, by drawing fibres of PMMA it is possible to align the molecular chains in the fibre direction for improved mechanical properties. They showed that the incorporation of these fibres to the bulk PMMA significantly improved its mechanical properties as well. On the other hand though, when these fibres were chopped and randomly oriented, they had no advantage over the unmodified polymer in terms of strength (Jagger and Harrison 1999).

Higher abrasion resistance of PMMA may be expected to be achieved with the addition of cross-linking agents like ethylene glycol dimethacrylate (EGDMA) or triethylene glycol dimethacrylate (TEGDMA) to the liquid part of the mix. Cross-linkage should result in an increase of the molecular weight of the material. However, Harrison et al (1978) found that the addition of a cross-linking agent had no significant effect on the properties of abrasion resistance and impact strength of PMMA. They attributed this finding to the geometrical limitations of the polymer network preventing $100 \%$ efficiency of crosslinking. The unreacted cross-linking agent in the form of a residual monomer or pendant chains can plastisise the polymer and affect its mechanical properties.

In all cases any resulting increased strength of reinforced PMMA may contribute to the resistance of the material to abrasion. The resultant increase in elastic modulus causes the material to deform little under occlusal stress, leading to localised destruction where an antagonistic contact exists. This localised destruction serves as an inherent crack arresting mechanism by retarding neighbouring cracks near the surface of the material being joined together (Braem et al 1994a). 
Acrylic resins appropriate for veneering are most commonly available in either heat or cold cured forms. In heat cured form, the curing cycle is designed to raise the temperature to a point at which sufficient free radicals are produced from benzoyl peroxide and polymer chains form by free radical polymerisation. In cold cure form a reducing agent is added to the monomer. This reducing agent is often a tertiary amine which reacts with the benzoyl peroxide initiator at room temperature to produce the free radical polymerisation of the monomer. The different components taking part for the formation of a free radical are shown in Figure 2.1.

Heat cured acrylic resins have a higher degree of conversion resulting in greater crosslinking than with cold cured resins (Ruyter and Øysæd 1982a). This is due to the fact that the degree of conversion caused by a chemical activator is not as high as that produced by heat activation (Baker et al 1988, Vallittu et al 1995b). The greater elastic modulus associated with heat cured resins, results in less deformation in function. They are generally more brittle. The residual monomer content of cold cured resins may be four times greater than that of correctly heat cured resins (McCabe and Basker 1976). Further, it has been suggested that the porosities in the structure of cold cured PMMA may affect the amount of water sorption and release of monomer by enhancing its diffusion between PMMA and water (Vallittu et al 1995b).

High residual levels of monomer are a potential danger to the patient as sensitisation may occur. The monomer can be absorbed by the tissue with which it is in contact. It will also affect the mechanical properties of the material by plasticising the polymer. The modulus of elasticity, hardness and strength at failure are all reduced since less cross-linking has taken place to form the polymer (Asmussen 1982b, Stafford and Brooks 1985). 

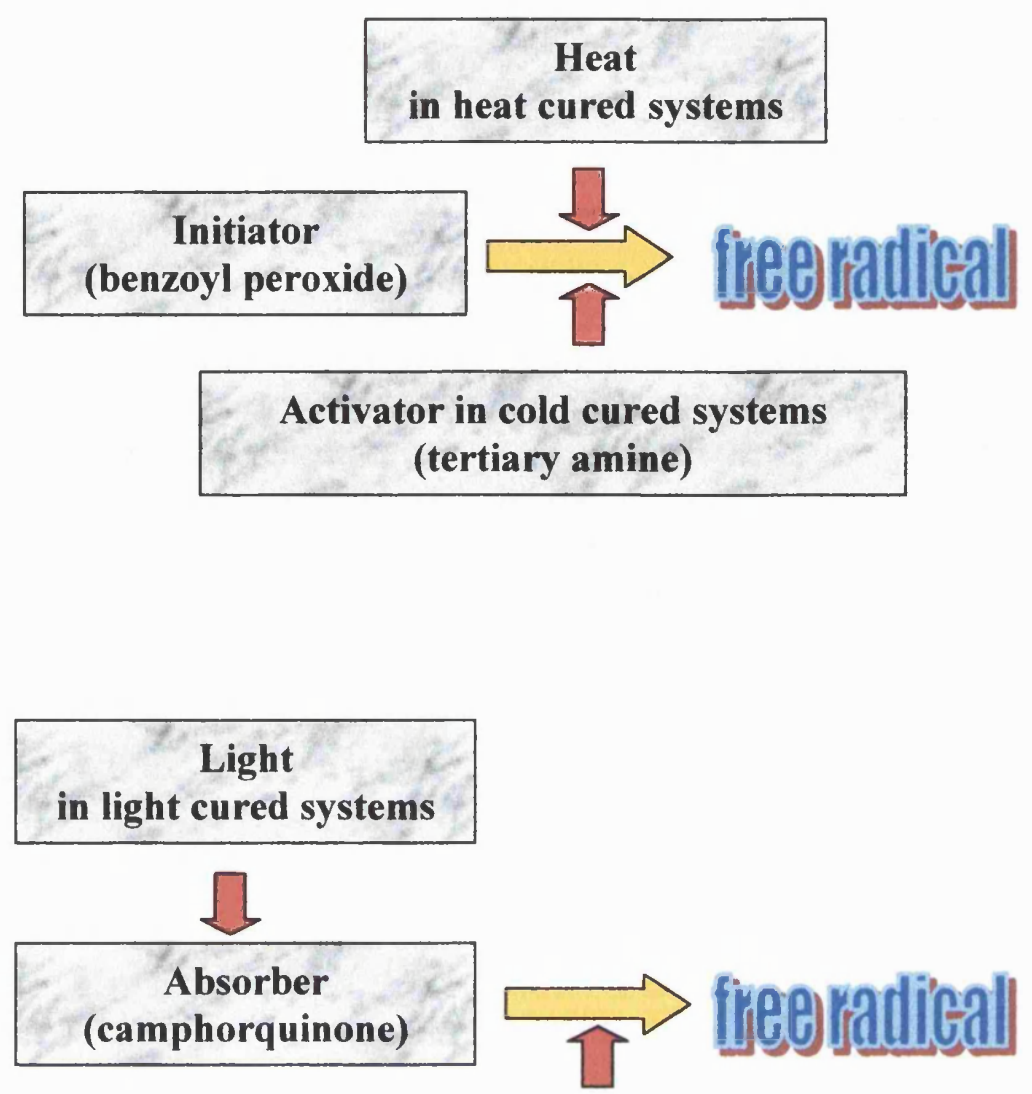

Activator in light cured systems

(tertiary amine)

Figure 2.1 : Simplified representation of the formation of a free radical in heat, cold and light cured systems

Acrylic resins are also available in light activated forms, or as preformed teeth. Light cured resins, show a high degree of conversion when compared to cold cured resins. This may be due to the fact that hand mixing may increase bulk porosity. This in turn may contribute to increased bulk inhibition of polymerisation and increased potential for monomer leaching (Eliades et al 1995). Also, hand mixing will increase the entrapped oxygen within the mix. Oxygen acts as an inhibitor in free radical addition polymerisation causing more residual monomer to be present and less conversion of double bonds (Vallittu 1999c). 
Triad VLC ${ }^{*}$ is a light cured denture material, which compares well with heat cured material in terms of impact strength and hardness. This is mainly due to the resin being a silica filled dimethacrylate based material. It has a higher elastic modulus (Smith et al 1992) and superior accuracy of fit (Smith and Powers 1992) but shows over three times more water sorption (Khan et al 1987). Its advantage lies in the decreased polymerisation shrinkage exhibited and in its ease of use.

Artificial teeth were originally made with PMMA. More recently new types have been produced using a range of dimethacrylate resins which appear to provide better properties than the traditional ones. Trubyte Bioform IPN*, with an unfilled, highly cross-linked, interpenetrating polymer network or SR-Orthosit-PE**, composed of $100 \%$ cross-linked urethane dimethacrylate and a small hydrophobic silicon dioxide microparticle filler are examples of the more recently developed types. These materials are significantly more wear resistant than conventional acrylic teeth and with improved mechanical properties (Ogle et al 1985, von Fraunhofer et al 1988, Whitman et al 1987). However, these highly cross-linked resins containing fillers, are very similar to highly filled composites in their behaviour and tend to show a brittle behaviour (Abe et al 1997).

\subsection{COMPOSITE RESIN}

Phillips (1981) defined a composite as a three dimensional combination of at least two chemically different materials with a distinct interface separating the components. Generally, compared to previous products of the 1980's, modern composites are heavily filled. They have lower polymerisation shrinkage, a lower coefficient of thermal expansion, higher compressive strength and stiffness. Depending on the filler materials

\footnotetext{
Dentsply Int. Inc., York, PA, USA

** Ivoclar, Schaan, Liechtenstein
} 
used, greater radiopacity than unreinforced resins can be achieved. Compared with PMMA, composites have a higher elastic modulus, lower solubility, higher tensile and compressive strength together with improved wear resistance. All these factors are a result of the inert filler to the resin. The filler is generally coated with a coupling agent.

The strength of the resin composites is dependent upon:

a) monomer composition,

b) filler content,

c) degree of conversion and cross-linking of the resin following polymerisation

(Carvalho et al 1996).

The handling characteristics of the composites are also advantageous since light curing permits extended manipulation time prior to polymerisation. Refurbishments and additions can be easily undertaken at the chair side. Some of the properties are derived from the resin matrix and others are related to the filler and the coupling agent. The resin matrix binds the filler particles into a cohesive mass. It also controls the colour of the composite due to inorganic oxide pigments. Darkening of cold cured composites is often observed when the tertiary amine activator oxidizes, producing coloured products. Similar discolouration may also occur with light activated materials. However, the amine concentration is optimised and nearly all is used up in the reaction. Strength, stiffness, abrasion resistance and coefficient of thermal expansion are controlled by the filler (Söderholm 1984a, Braem et al 1989, Talib 1993). Nevertheless these properties are also influenced by the various additives of the matrix that promote or inhibit polymerisation or help stabilise it. 


\subsection{THE ORGANIC PHASE (MATRIX)}

In the search for a composite resin matrix material, methyl methacrylate (MMA) was found to be unsuitable. As described previously, it had a high polymerisation shrinkage, setting exotherm and high coefficient of thermal expansion. It also caused/sensitivity t $^{a}$ reaction in some patients (Paffenbarger et al 1953). The shrinkage in a composite resin can be a problem with inherent stresses being set up between the matrix and filler particles and similarly between tooth and restoration. The higher thermal expansion can also result in appreciable marginal leakage (Combe et al 1999).

Bowen tried to develop materials without these drawbacks. In 1962, he synthesised an experimental dimethacrylate monomer by the reaction of bisphenol $\mathrm{A}$ and glycidyl methacrylate. The acronym given to the new monomer was bis-glycidyl methacrylate (Bis-GMA).

Bis-GMA has the reactivity and easily controlled setting properties of MMA and the strength and low shrinkage of epoxy resin. It contains an aromatic backbone with reactive methacrylate end groups. This bulky monomer is superior to methyl methacrylate because of its large molecular size and chemical structure, providing lower volatility, lower polymerisation shrinkage, more rapid hardening and the production of stronger and stiffer resins. Its setting reaction proceeds by addition polymerisation as with MMA (Craig 1981). Addition of a filler produced a composite with good mechanical properties. However, it had a very high viscosity and in order to obtain a reasonable consistency for clinical handling, it was diluted by addition of other methacrylate monomers of lower molecular weight and lower viscosity (Bowen 1963). 
Most resins will be cross-linked with EGDMA or TEGDMA (Yoshida et al 1992), to reduce their viscosity to an appropriate level to allow incorporation of filler particles (Asmussen and Peutzfeldt 1998). The addition of TEGDMA improves the mobility of monomer molecules and increases the degree of conversion (Tarumi et al 1999). However, dilution of the monomer particularly Bis-GMA, has negative effects such as increased polymerisation shrinkage. When TEGDMA is added at a low concentration substituting Bis-GMA in the monomer composition, the flexural strength also increases since the degree of conversion is increased. However, as the concentration of TEGDMA increases, so the flexural strength decreases since the TEGDMA monomer is more flexible compared to the Bis-GMA one (Asmussen and Peutzfeldt 1998).

During polymerisation, resin systems shrink mainly because of the formation of a macromolecular chain network from discrete monomer species involving conversion of intermolecular distances of 0.3-0.4 nm into primary, covalent bonds with lengths of about $0.15 \mathrm{~nm}$. The extent of polymerisation shrinkage depends, among other things, on the molecular weight and functionality of the monomers (Peutzfeldt 1997). Thus, comparing monomers of the same molecular weight, polymerisation shrinkage increases when functionality increases. Comparing monomers of the same functionality, polymerisation shrinkage increases when molecular weight decreases. Consequently, dilution of BisGMA increases the polymerisation shrinkage (Asmussen 1975).

A number of resin systems based on urethane dimethacrylate (UEDMA) have also been developed. This monomer has been used alone or in combination with other monomers e.g., Bis-GMA and TEGDMA (Vankerckhoven et al 1981, Ruyter and Øysæd 1987, Ruyter and Sjøvik 1987). The advantages of UEDMA resins have been reported to be lower viscosity and greater flexibility of the urethane linkage which may improve 
toughness (Indrani et al 1995, Peutzfeldt 1997). It seems that the urethane linkage is at the same time relatively flexible and strong (Asmussen and Peutzfeldt 1998). However, differences between resin composites based on UEDMA or other urethane dimethacrylates and conventional Bis-GMA-based resin composites are difficult to confirm from existing in vitro and in vivo studies (Raptis et al 1979, Knudsen and Jørgensen 1983, Freilich et al 1992, Mair 1995). One reason for this difficulty is that differences caused by dissimilar monomer systems are camouflaged by the fact that the materials differ in many other respects, e.g., type and amount of filler, type and amount of initiators, and quality of silanisation of the filler particles.

Copolymers of alicylic spiroorthocarbonates (SOCs) and epoxies that would form hard non-shrinking matrix resins suitable for formulating dental composites, have been developed (Eick et al 1993a+b). These non shrinking matrixes are still in the experimental phase, but may potentially result in set composites that are adapted better to a metal substrate when used for veneering since there would be no risk of contraction away from the metal surface during light curing. The expansion of SOCs on polymerisation was first developed by Bailey (1975), who demonstrated their usefulness for some high strength industrial composite resins. An alicylic SOC consists of a total of four rings, two on each side of the spiro carbon (Bailey 1990).

Veneering the different components of an RPD may demand resin composites of different mechanical properties. Undoubtedly, for some purposes the materials should be stiff and strong. In other situations though, flexibility is more important and strength is not a critical factor. Toughness or resilience may also be of interest. By judicious choice of the relative content of Bis-GMA, UEDMA, and TEGDMA and in conjunction with proper 
selection of filler as it is described below, resin composites may be designed to fulfil specific applications.

\subsection{THE DISPERSED PHASE (FILLER)}

By "filling" a resin, its physical and mechanical properties are markedly affected. There are several features of the filler, such as filler chemistry, quantity, particle size, shape and distribution that are important with respect to the mechanical properties of a composite (Willems et al 1993, Leinfelder 1995). Wear and polishability of composites in the oral environment are primarily related to filler particle quality, size and interparticle spacing (Jørgensen and Asmussen 1978, Bayne et al 1992, Ferracane 1995, Suzuki et al 1996). As the matrix is softer and less wear-resistant than the inorganic filler, it is preferentially abraded by both two or three body wear. When the polymer matrix wears down, it exposes the filler particles, allowing them to be plucked from the surrounding matrix during the next abrasion cycle.

Large hard particles transmit considerable stresses to the matrix, possibly resulting in microcracking and subsequent loss of material. In contrast, a reduced load per particle ratio results when a large number of small particles is present per unit volume. When these small particles are incorporated in large quantities so as the amount of filler particles per volume is increased, and are of a spherical shape, the inter particle distance is decreased (Kim et al 1994). Therefore, the use of a large volume of smaller particles minimises the extent of surface degradation during chewing, since there is a decreased amount of resin exposed to the environment, thus reducing the rate of abrasive wear (Bayne et al 1992, Craig 1997d, Mante et al 1993, Leinfelder 1995). 
A clinical study of five years has confirmed the success of many composites in small to moderate posterior occlusal cavities, showing wear rates of $10 \mu \mathrm{m}$ or less per year, when the average particle size is less than $1.0 \mu \mathrm{m}$ (Mazer and Leinfelder 1992).

Filler particles can degrade and debond more easily from the resin matrix after they have been saturated in water and cyclically stressed particularly if they are not silane treated (Söderholm 1981 and 1983). In an attempt to improve the mechanical properties and polishability of composite resins, microfilled composites were produced. In these materials, prepolymerised blocks of filled resin were added to the resin matrix of the composite (Lambrechts and Vanherle 1983). The procedure involves filler particles (size 40-400 nm) being added to a resin matrix which has been diluted with a volatile solvent. The solvent is evaporated and the remaining material is polymerised. After polymerisation, the set blocks of resin with seeds of silica are ground up and then added to the unpolymerised resin of the composite. In this procedure though, the large prepolymerised blocks are not silanated. The prepolymerised blocks are only partly bonded to the resin matrix and can debond and separate when subjected to the continuous application of localised stresses. These prepolymerised particles are partly held in the resin matrix by mechanical retention rather than by chemical means. Consequently, the interfacial bond is weak when exposed to occlusal loads (Suzuki and Leinfelder 1993). The resultant subsurface damage can then lead to bulk fractures (Kawai and Leinfelder 1995). This mode of failure is characteristic of most microfilled composites containing prepolymerised filler particles. More recently it has been considered that the resin matrix will cross-link with any residual monomer in the filler.

Within limits, the higher the volume fraction of filler is, the higher the mechanical properties become. Tensile strength, elastic modulus (Braem et al 1986b, Davis and 
Waters 1987), and hardness values (Williams and Johnson 1993, Willems et al 1993) are all increased. High filler loading also contributes to reduced polymerisation shrinkage, water sorption and a decreased coefficient of thermal expansion (Talib 1993, Carvalho et al 1996). The filler chemistry also contributes to the amount of water sorption, with composites containing quartz and barium particles showing less water uptake compared to the ones that contain silica (Calais and Söderholm 1988).

To achieve high filler fractions, the viscosity of the monomer has to be lowered and this is often accomplished by incorporating diluents into the monomer mix. A balance has to be struck in between diluting the monomer mix and maximising the filler fraction for optimum properties. Excess dilution will increase the polymerisation shrinkage of the composite. Overpacking it with fillers, might also result in a material containing only partially wetted filler particles that will show poor physical and mechanical properties and exhibit early failure (Talib 1993). It is essential that the material forms a cohesive mass. Failure to acknowledge this leads to rapid break up of the material.

Microfilled composites with lower filler content, have been traditionally precluded for stress-bearing applications, because they exhibit low elastic modulus and low resistance to deformation (Braem et al 1989). On the other hand though, when the elastic modulus of hybrid composites becomes too high, the delicate equilibrium between brittleness, tensile strength and compressive strength is disturbed. Brittle failure within the composite material can lead to the composite veneer being discoloured. Failure at the interface with the metal substrate of the partial denture, can lead to the veneer breaking off.

The fillers that have been used in dental materials are generally derived from quartz, aluminum silicate, borosilicate glass, barium glass and strontium glass. Depending/the 
type of filler particles, the wear properties of the composite can also be modified. Quartz filler particles with a size distribution skewed to smaller sizes and with a large proportion $1 \mu \mathrm{m}$ or less can be selected for good wear resistance. Composite resins filled with zirconium silica, as in the case of $\mathrm{Z} 100^{*}$, or quartz are considered to be more resistant to wear compared to composite resins containing microfilled, barium silica or colloidal silica as in the case of Silux Plus* (Suzuki et al 1996).

Changes in the filler component of commercial composites have prompted the periodic development of classification systems for dental composites based upon the filler size and volume fraction.

- In 1983, Lutz and Phillips published a system for characterising dental composites based on particle size. Their system included conventional or traditional composites (average particle size 1-15 $\mu \mathrm{m}$ ), hybrids containing a mixture of ground glass and microfill particles (average particle sizes $5 \mu \mathrm{m}$ and $0.04 \mu \mathrm{m}$ respectively), and microfills (average particle size $0.04-0.1 \mu \mathrm{m}$ ).

- Willems et al (1992) published a similar classification system. They emphasised that most popular composites have smaller mean particle sizes and fewer large particles than composites in the 80 's. They claimed that the term "hybrid" should no longer be used, since nearly all modern dental composites are "hybrids" of two size ranges. Instead, they classified composites as densified, microfine, traditional and fibre-reinforced. Densified composites were further classified as midwayfilled ( $<60$ vol\%) and compact filled ( $>60 \mathrm{vol} \%)$, with classifications of ultrafine

*3M Dental Products. St.Paul, MN, USA 
(average size $<3 \mu \mathrm{m}$ ) and fine (average size $>3 \mu \mathrm{m}$ ) within each category. Microfine composites were classified according to the type of prepolymerised filler particles that they contain.

- Another classification has been described by Bayne et al (1994) in which the composites are classified by the size of their largest fillers as microfills (average size $0.01-0.1 \mu \mathrm{m}$ ), minifills (average size $0.1-1.0 \mu \mathrm{m}$ ), midfills (average size 1.0$10.0 \mu \mathrm{m}$ ), macrofills (average size $10-100 \mu \mathrm{m}$ ) and megafills (average size $0.5-2$ $\mathrm{mm})$.

- A very simple classification has been adopted by Craig (1997c) in which all current composites can be classified as fine, microfine or hybrid. Fine composites have 0.5-3 $\mu \mathrm{m}$ particles, microfine have 0.04-0.2 $\mu \mathrm{m}$, and hybrids have a blend of both fine and microfine particles. This classification is routinely used nowadays due to its simplicity. According to this classification Silux Plus is considered a microfine (particle sizes $0.04-0.09 \mu \mathrm{m}$ as per manufacturer information) and Z100 is considered a hybrid material (particle sizes $0.01-3.5 \mu \mathrm{m}$ as per manufacturer information). A representation of the typical appearance of a microfine composite containing prepolymerised particles similar to Silux Plus and of a heavily filled hybrid composite similar to $\mathrm{Z100}$ can be seen in Figures 2.2 and 2.3 respectively. 


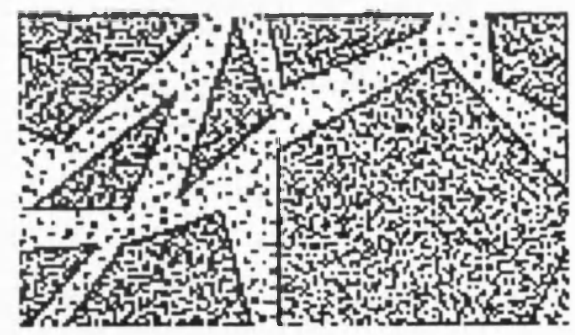

Figure 2.2 : Schematic illustration of the structure of a microfine composite containing prepolymerised particles (black dots illustrate colloidal silica particle

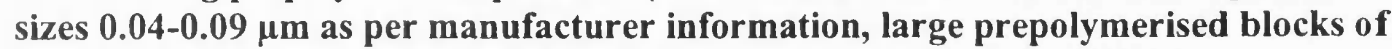
average $22 \mu \mathrm{m}$ (Willems et al 1993) are also illustrated )

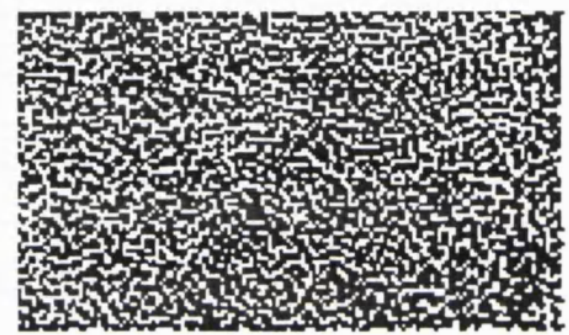

Figure 2.3 : Schematic illustration of the structure of a hybrid heavily filled composite (black dots illustrate zirconium silica particle sizes $0.01-3.5 \mu \mathrm{m}$ as per manufacturer information)

\subsection{THE INTERFACIAL PHASE (COUPLING AGENT)}

In order for a composite to have substantially improved mechanical properties, transfer of stress under loading from the high strength, dispersed, reinforcing filler through the more ductile polymer matrix should occur (Jones and Rizkalla 1996). For this transfer to be effective, a good bond must exist between the polymer matrix phase and the inorganic reinforcing phase. This requirement is accomplished by the use of a coupling agent that attaches to the inorganic reinforcing phase and reacts with the organic phase. The usual coupling agent for dental composites is a molecule which has silanol ( $\mathrm{Si}-\mathrm{OH})$ groups on one end and methacrylate groups (containing $\mathrm{C}=\mathrm{C}$ ) on the other end. These molecules are 
capable of forming bonds to both the silicon-based fillers and the methacrylate groups in the resin matrix. These bonds can be seen in Figure 2.4. They are commonly used in hybrid composites but not on the prepolymerised particles of microfine composites since some chemical reactions will occur between prepolymerised resin blocks and the monomer during polymerisation.
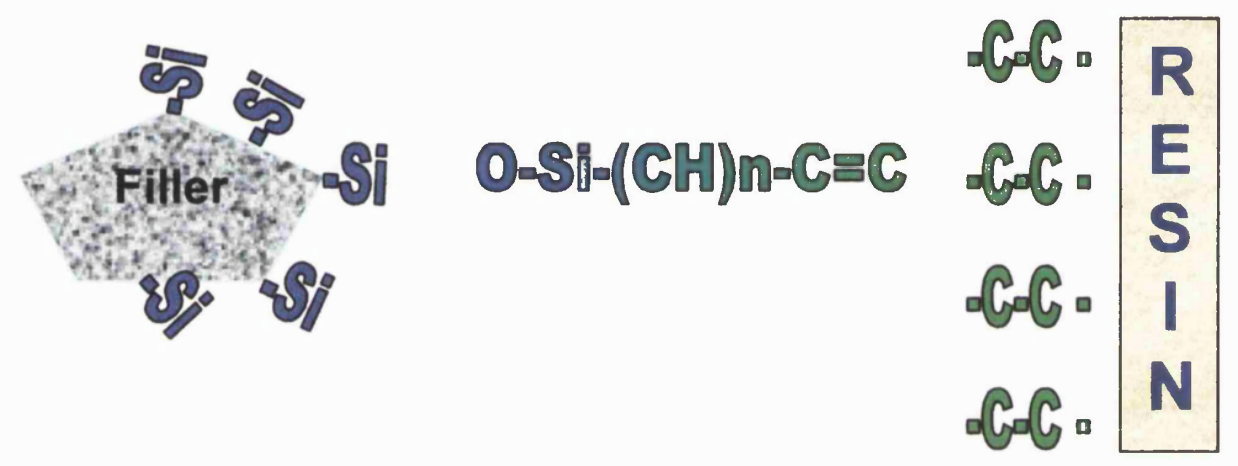

Figure 2.4 : Simplified schematic representation of silane bonding between the fillers and the polymer matrix in dental composites

Studies have verified the importance of this coupling in dictating the mechanical properties of the composite by influencing its tensile strength and water sorption (Nishiyama et al 1991, Mohsen and Craig 1995a+b). Most commercial composites contain silica-based fillers, and therefore use silanes with functional methacrylate groups as filler/matrix-coupling agents. The most common silane is 3-methacryloxypropyl trimethoxysilane (MAPM). Other agents have been evaluated, such as other silanes and various titanates and zirconates, but none has been as successful as MAPM (Mohsen and Craig 1995a+b). Further developments in fillers for composites may necessitate the continued development of coupling agents. Although the methods used by the manufacturers to silane the filler surface are trade secrets, further studies are needed of how to optimise the silane treatment procedures of dental composites in terms of optimal 
amounts and modes of coverage (Söderholm and Shang 1993). Frequently, only a proportion of the surface area of the filler is coated. Further the silane coupling agent can be hydrolysed with time causing degradation of the bond between resin and filler.

Other components of the matrix include polymerisation initiators, inhibitors and stabilisers:

\subsection{POLYMERISATION INITIATORS}

The polymerisation reaction can be chemically initiated with the reaction of a peroxide initiator and an amine activator (e.g. N,N- dihydroxyethyl-para-toluidine) or it can be initiated with a beam of light of appropriate wavelength in the presence of a light sensitive absorber and an activator (Figure 2.1).

Light-cured resin composites offer many clinical advantages over their chemically cured equivalents but their polymerisation depends completely on adequate delivery of light energy i.e the intensity of irradiation and its duration (Caughman et al 1995). Initially, UV light of $360 \mathrm{~nm}$ wavelength and different initiators were used. Concerns were expressed about the safety aspects of the use of high intensity UV light and avoidance of these problems has been facilitated by the introduction of visible light activated systems that emitted at wavelengths in between 400-515 nm (Shortall et al 1995, Shortall and Harrington 1996). There is still a need to be cautious in the use of these systems too as the use of the high-intensity light itself can harm the retina (Antonson and Benedetto 1986). An added advantage to the visible light activated composites is that the depth of cure achievable with UV light is considerably less than that obtained with visible light (Ruyter and Øysæd 1982b, Leinfelder 1993). 
When visible light became the preferred activation energy source, diketones and aromatic ketones that can absorb light in the blue range $(460 \mathrm{~nm}$ ) were employed (Dart and Nemeck 1978). A tertiary amine is also present as an activator. A common initiator for visible light curing is camphorquinone, with peak absorption between $460-470 \mathrm{~nm}$.

\subsection{POLYMERISATION INHIBITORS}

These additives are used to stabilise the composite until its polymerisation is desired. Hydroquinone and the methyl ether of hydroquinone are the most common examples of added inhibitors that ensure the material's shelf life, by preventing the monomer undergoing spontaneous polymerisation. They also ensure enough working time.

\subsection{STABILISERS}

These additives are used to diminish discolouration of the resin during subsequent aging.

The aging effect is dependänt on the amount of residual amine present in the resin. In order to reduce this yellowing effect that occurs slowly with time in composites, stabilisers such as benzophenones and benzotriazoles are used. Pigments are also used to produce veneering composite resins to match the colour of the dental tissues being restored. Iron oxides are preferred to the organic dyes for their superior colour stability.

\subsection{DEVELOPMENTS IN THE POLYMERISATION OF COMPOSITE RESINS}

The polymerisation of dimethacrylate Bis-GMA forms an organic matrix of a three dimensional cross-linked network containing residual methacrylate groups. In most light cured resin composites, not all of the methacrylate groups of the dimethacrylate monomers are reacted in the polymerisation process (Ruyter and Svendsen 1978); unreacted methacrylate groups can amount to $23-43 \%$ in the cross-linked polymer increasing the water sorption of the material (Asmussen 1982a). There is also good 
correlation between hardness and quantity of unreacted methacrylate groups remaining in the polymer (Asmussen 1982b). Modifying the curing process to extend the degree of polymerisation has led to resin polymers with a greater wear resistance, higher elastic modulus and less water sorption.

Air, because it contains oxygen, has a strong influence on the clinical behaviour of dental composite resins (Feilzer et al 1993). It is the atmospheric oxygen that partially interferes with the curing process. Small quantities of dispersed air in the unused composite resin are necessary to prevent partial curing during storage. It can however act as a means of weakening of the structure if incorporated into the resin phase during placement. Resin in contact with an air void will be inhibited from reaching its maximum curing potential (Feilzer et al 1993). Entrapped oxygen is also important as it relates to wear resistance (Leinfelder 1985). When air inhibited regions become exposed through the attrition or degradation process normally associated with masticatory forces, the material should wear away at a rate appreciably faster than that when air defects are absent, as its hardness value decreases (Asmussen 1982b). Air entrapment also affects the fatigue strength of the composite, since the presence of defects such as air bubbles may cause stress concentration and favour catastrophic failure (Braem et al 1994a). This rationale can also explain why the wear resistance of light cured systems is often superior to that of cold cured systems. For cold cured systems, the mixing of two relatively viscous pastes as a base and catalyst inevitably incorporates air into the material. On the other hand thbdgh, it has been suggested that air entrapment within the composite reduces the polymerisation stress due to the resultant oxygen inhibition or to the increased free surface area formed by the pores within the bulk of composite (Feilzer et al 1993). 
Oxygen also plays an important role in the apparent translucency or opacity of the cured resin restoration, too. Its presence, as an entrapment, in the final restoration, regardless of dimension, tends to break up or diffract the natural light as it reflects from the surface of the restoration. Removing all of the trapped air causes the restoration to become considerably more translucent.

The technique of curing the resins at an elevated temperature under pressure in the absence of oxygen, has been used primarily for the construction of inlays, onlays and crown restorations in conjunction with a metal substrate. For the reasons described, it was hoped that extraoral polymerisation, in the absence of oxygen would increase the longevity of the composite resin, and improve the mechanical properties and wear resistance by minimising the effects of polymerisation shrinkage, while maximising the degree of conversion. The inlay technique, which involves a post curing high temperature cycle, appeared to be an effective mechanism for increasing the degree of conversion by a second heat cured cycle (Asmussen and Peutzfeldt 1990, Ferracane et al 1995). However, it does not seem to have a significant effect on the clinical performance (McCabe and Kagi 1991). In fact, the material may exhibit brittle behaviour under flexural stress as more cross-linking can occur. The high degree of conversion achieved using "improved" polymerisation methods might actually hinder the clinical performance and durability of the composite veneer of an RPD specially if it is employed in components of the prosthesis that are prone to flexural stresses. i.e. flexible portions of the retaining clasps. 


\subsection{METAL-RESIN BONDING}

The polymer resin veneer of an RPD needs to be retained to the metal substructure. Metal-resin bonding, can be either classified as mechanical or chemical. It is used in removable prostheses to retain acrylic saddles or facings to metal substructures. This permits:

a) attachment of tooth coloured resins to metal supporting structures

b) reduced leakage and staining at the metal-resin junction

c) improved resin retention and improved fracture resistance.

Mechanical and chemical bonding may often be combined clinically for increased retention.

\subsubsection{MECHANICAL RETENTION}

Mechanical bonding, can be further subdivided into macromechanical retention which relies on visible undercuts and micromechanical retention which employs microscopic porosities in the metal/alloy.

\subsubsection{MACROMECHANICAL RETENTION}

Dunny and King (1975), examined the effect of different retention designs, on the strength of retention of acrylic resin. They concluded that the design of the framework retention form, is an important element in determining the strength of the attachment of acrylic resin to the metal denture base.

There are a number of different retentive elements that have been used over the years in RPDs including perforations, meshwork, (Shen and Torres 1985, Taleghani et al 1987) and protrusive elements such as nail heads and beads (Tanaka et al 1978, Shue et al 1987). Metal perforations had the advantages that they were simple to design and they could be used with any metal. 
The incorporation of beads is one of the oldest and simplest methods of achieving mechanical retention with any alloy. Nicholls and Shue (1986), showed that small beads result in higher tensile bond strengths and therefore are preferable to larger ones. Tanaka et al (1978) in comparing a range of beads, concluded that $0.18 \mathrm{~mm}$ diameter beads offered the greatest retention. Potentially though, the presence of beads may hinder the uniform application of resin to the metal surface and also add a considerable thickness to the prosthesis which is of significance in areas of limited interocclusal space. To compensate for this, the framework might need to be thinned, making it susceptible to cracking by metal fatigue.

In all the above methods there is no chemical union between the resin and the metal. When thermo-cycled the interface is continuously subjected to stress, resulting in the interface being opened and microleakage occuring (Tulunoğlu and Oktemer 1997). Apart from discolouration of the interface being caused, there is a risk of crack initiation and propagation due to the localised stress concentration at the interface.

\subsubsection{MICROMECHANICAL RETENTION}

Micromechanical retention is achieved by creating micro-roughness on the alloy surface, which increases the area for bonding with minimum invasion of the interarch space. It is now used routinely and provides more satisfactory bond strengths than those reported for macroscopic retentive designs (Leinfelder 1994).

\subsubsection{PAST TRENDS OF METAL SURFACE PREPARATION}

The electrolytic etching of base metal alloys, used to be one of the most popular methods of preparing the metal surface and was used routinely for resin retained restorations (Livaditis and Thompson 1982, Ekstrand and Ruyter 1987). However, the process 
requires sophisticated equipment and a technical procedure that demands careful control by the technician, with microscopic evaluation of the quality of the etch (Hussey et al 1989). Contamination of the etched metal surface, often resulted in an extra visit for the patient, since re-etching was required. Even handling the etched surface can cause damage.

Chemical etching was accomplished by immersing the casting in a freshly mixed chemical solution (Love and Breitman 1985). Different concentrations of different acids for different time intervals have been reported in the literature. Love and Breitman (1985) favoured the use of a mixture of nitric acid, methanol and hydrochloric acid. Etching regimes include either the application of an etching gel (Met-Etch gel ${ }^{*}$ ) for 3 minutes at $150^{\circ} \mathrm{F}$ and then reapplication for 7 to 10 minutes (Doukoudakis et al 1987) or application of the gel for 20 minutes at room temperature (Re et al 1988).

Overall, chemical etching provides more reliable results than electrolytic etching due to the simplicity of the procedure (Livaditis 1986). It can be carried out by less experienced personnel, the equipment is cheaper, readily available to laboratories and allows several restorations to be etched at the same time, unlike electrolytic etching, where only one restoration can be etched at a time. On the other hand though, the solutions used are dangerous and irritant in contact with the skin, therefore/care is needed handling them.

\subsubsection{CURRENT TRENDS OF METAL SURFACE PREPARATION}

Today, electrolytic and chemical etching have been largely superseded by sandblasting, used in conjunction with chemical bonding agents. A number of adhesive systems use a resin luting cement or a coupling agent applied to the oxide layer present on sandblasted

\footnotetext{
- Gresco Products, Inc., Stafford, Tex., USA
} 
metal surfaces. Sandblasting, as with etching, roughens the surface of most metals and increases the surface area available for bonding. It has been suggested, that when alumina is used for sandblasting, complex reactions occur on the alloy surface resulting in a highly activated surface, which is apparent from the increased wettability by luting material (Kern and Thompson 1993a, Chung and Hwang 1997). The exact process though, is poorly understood at present. Both mechanical and chemical factors might play a role (Coelho et al 1996). It seems, that although the sandblasted metal surfaces are ultrasonically cleaned prior to bonding to reduce the oxide thickness, some positively charged alumina remains on the surface of the metal and ionically bonds to the negatively charged active group of the resin adhesive. Kern and Thompson (1993a), suggested that the alumina content of base alloys increased $37 \% \mathrm{wt}$, after sandblasting and that ultrasonic cleaning removed only a small amount of the embedded alumina.

The preferred size of the alumina oxide particles used, differs from report to report. $50 \mu \mathrm{m}$ grit, which cleans the surface sufficiently and does not remove too much metal, something that can affect the fit of blasted restorations, is favoured by some investigators and has been recommended by most manufacturers (Tanaka et al 1986, Ferrari et al 1987, Pegoraro and Barrack 1987, Kountouras et al 1999). Others, though, have used $150 \mu \mathrm{m}$ grit or $250 \mu \mathrm{m}$ particle size (Wiltshire 1986, el-Sherif et al 1991, Barclay and Williams 1994). Barclay et al (1997) have reported though, that although the three dimensional surface of the metal is different with different grit sizes, the overall differences in the contact surface area are very small, with little if any significant differences on the bond strength values. 


\subsubsection{CHEMICAL BONDING}

Adhesion is the attachment of materials in contact that resists the forces of separation. Mechanical adhesion between resin and metal has already been discussed. Chemically, there are two major ways that a resin can bond to metal, either interfacially or adhesively. Interfacial bonding uses an intermediate interface, such as tin plating or ceramic coatings fused to the metal surface, such as Silicoating ${ }^{*}$ and Rocatec ${ }^{* *}$. Adhesive bonding employs chemically attached adhesive groups to the treated metal surface.

\subsubsection{INTERFACIAL BONDING}

\subsection{TIN PLATING}

Sandblasting by itself, results in lower bond strengths for noble alloys bonded to a resin material compared to base metal ones (McConnell et al 1989, Hofstede et al 1990). Tin plating is a conditioning procedure prior to using adhesive cements. Tin forms more organic complexes than any other metal and can result in an increase in bond strength when cements such as Panavia $\mathrm{X}^{* *}$ are used (Hofstede et al 1990, Gates et al 1993, Imbery and Davis 1993). Tin plating of noble alloys, after sandblasting, produces a roughened surface forming tiny crystals on the surface, which enhance the micromechanical bond achieved and retention of the luting cement. Chemical attraction has also been suggested to occur, through hydrogen bonding to the tin oxide formed (McCaughey 1993).

\footnotetext{
"Hereaus Kulzer, Wehrheim, Germany

"Espe, Seefeld, Germany

*** Kuraray Co Ltd., Osaka, Japan
} 


\subsection{POROUS METAL COATINGS}

Porous metal coatings, can also offer micromechanical retention to alloy surfaces (Hanson et al 1983). Some systems like Inzoma P900 $\mathrm{TM}^{*}$ fire a $20-30 \mu \mathrm{m}$ coating of porous metal onto a cast metal (Stokes and Tidmarsh 1987). This creates a porous outer layer on the metal surface which could engage resin bonding materials. Stokes and Tidmarsh (1987) have reported bond strengths of $15.4 \mathrm{MPa}$ for treated palladium rich alloys. However, this procedure is highly technique sensitive.

\subsection{SILICOATER PYROGENIC SILANIZATION}

In pyrogenic silanization, a thin bondable glass interface containing silica oxides is heat fused to any metal surface, including noble alloys. The metal surface is blasted first and then coated with a solution (Siliclean). Silica oxides are deposited in the metal surface by heating with a flame in a specially designed apparatus. A silane and a resin coat are then applied for protection during transportation (Laufer et al 1988, Caeg et al 1990). Silicoating provides intial bond strengths of resin to metal which is reported to be $25 \%$ higher than electrolytically etched alloys (Re et al 1988, Caeg et al 1990). It can be used to bond composite or acrylic resins to any metal surface.

Electrolytic etching of a metal surface prior to silica treatment, has been shown to produce a lower bond strength compared with silica treatment after sandblasting (Creugers et al 1988, Lüthy et al 1990). When etching was done after sandblasting prior silicoating, Caeg et al (1990) found bond strengths to/reduce. This reduction could be due to the removal of the high energy surface regions, produced by the sandblasting process, when etching was performed. Furthermore, the presence of deep microscopic crevices as a result of the etching procedure, may not allow adequate silica treatment.

\footnotetext{
- Ivoclar AG, Shann, Liechtenstein
} 
Smith et al (1993) showed that when non precious alloys were silica coated, a reduction in the bond strength values occurred after long term water storage and thermo-cycling. Nevertheless, Hansson (1990), when he compared the bond strengths of silicoated precious alloys to those of a $\mathrm{Co} / \mathrm{Cr}$ alloy, found out that silicoating showed a higher bond strength degree to the non precious alloy. Ishijima et al (1992) reported that this bond strength was more resistant to thermo-cycling compared to sandblasting alone. Variation in the experimental set-ups might account for the differences reported. However, when Pröbster and Henrich (1997) examined 11 year follow up data of resin bonded bridges, they concluded that compared to other means of retention, silicoated base metal alloys had the highest probability of survival. Disadvantages of using the silicoater method may include the uneven distribution of the flame over the casting and the thick coating of resin required. This cannot be altered. Also the procedure is costly and requires the need for highly trained personnel.

\subsection{SILICOATED MD SYSTEM}

This is a modification of the silicoater system from Kulzer, which required a special oven that burned a silica layer onto the surface. The difference between the techniques is that in the MD system, the metal surface is coated with silica oxides as a liquid and is fired at highly controlled and regulated temperatures. The system has the advantage of avoiding flame adjustment problems, eliminating the human factor. The principle of this technique is that chromium can form waterproof bonds with silicates. With the MD system a visible bonding layer is formed (Hansson and Moberg 1993).

\subsection{ROCATEC SYSTEM}

The system involves sandblasting with Rocatec-Plus, a special ceramic silicate particle material, which attachés silica particles to the metal surface by impact (tribochemical 
coating). The theoretic calculated speed of the Rocatec Plus particles hitting the alloy surface is $200 \mathrm{~m} / \mathrm{s}$, producing spot heating up to $1000^{\circ} \mathrm{C}$ as one form of energy is converted to another. A silane, Rocatec-Sil, is added to form a chemical bond between the embedded ceramic silicate layer and the resin. The Rocatec system is simple to use but expensive, it can be used for noble alloys, it does not require heating, but an increased volume of opacifying agents are needed, due to the darkening of the alloy surface (Leinfelder 1994, Özcan et al 1998).

The Rocatec system was also found to be superior in maintaining the bond strength values after water storage compared to Silicoater (MD) (Moulin et al 1999). Kourtis (1997) though, had completely opposite results. He found that Rocatec showed lower bond strengths compared to the rest of the systems after thermo-cycling. The extreme thermocycling conditions used in the latter study may account for the difference in the results reported.

\subsection{KEVLOC SYSTEM}

The Kevloc ${ }^{*}$ system, a relatively new system introduced in 1995 , is reported to offer a combination of mechanical and chemical bonding. Kevloc primer and Kevloc bond are applied to the blasted metal surface. The temperature needed for activation of the bonded layers is generated in the activation chamber of the Kevloc AC apparatus. It is proposed by the manufacturer that the silane which was previously used to chemically connect resin to metal is no longer necessary. This method though presented lower bond strengths when compared to Silicoating and Rocatec after short term thermo-cycling and water storage (Nergiz et al 1997). Vojvodic et al (1999) also found that bond strength values using the Kevloc method deteriorated after intense short term thermo-cycling as described

\footnotetext{
Hereaus Kulzer, Wehrheim, Germany
} 
by Hansson (1990) and Hansson and Moberg (1993). However the values reported were higher compared to silicoating, no marginal gaps were present at the interface and failure was of a cohesive nature within the opaque layers rather than adhesive.

\subsubsection{ADHESIVE BONDING}

Recently, adhesive systems have become very popular for bonding resins to metals. A major advantage of adhesives is that, many do not require special laboratory processes. They are quick and easy to use and they do not require etched metal. On the other hand, thought, they do not work well on all metals and the clinical research on the longevity of the bond is sparse.

Adhesive bonding resins, do not generally work well with gold alloys (Saito et al 1976), but with surface modifications such as tin plating, these adhesives are effective.

Most of the research done on the adhesive bond of resin to metal, has been directed toward the fixed cast restorations. Noble metals and $\mathrm{Ni} / \mathrm{Cr}$ alloys have been widely used for this research. In contrast to numerous studies on the resin bond strength to $\mathrm{Ni} / \mathrm{Cr}$ alloys, bond strength studies with adhesive systems on $\mathrm{Co} / \mathrm{Cr}$ alloys are limited (Kern and Thompson 1995). However, the structures of the $\mathrm{Co} / \mathrm{Cr}$ and $\mathrm{Ni} / \mathrm{Cr}$ alloys are very similar, resulting in them sharing similar surface characteristics and properties, as far as bonding to a resin material is concerned. Most of the present, commercially available, metal adhesive systems, have either a phosphate ester chemically active group, or a 4methacryloxyethyl trimellitate (4-META) group, which are chemically active within their composition. In removable prosthodontics, either chemically active denture base resin systems or inert veneering resins with chemically active bonding agents can be used to bond to a metal surface. 


\subsection{PHOSPHATE ESTER BASED SYSTEMS}

One of the most popular metal adhesive cements is Panavia $\mathrm{TM}^{*}$ which is frequently used to bond resin bonded bridges to teeth. Its been suggested by the manufacturer that the phosphate ester monomer 10-MDP (10-methacryloyloxy-decyl-dihydrogen phosphate) links to the resin though a long carbon chain, while also bonding to positively charged metallic ions on a metal alloy surface. The suggested form of bonding is shown in Figure 2.5. Sandblasting with aluminum oxide can produce the appropriate ions required for the bonding.

It has been suggested that phosphate ester bonding systems adhering to dentine hydrolyse after 6 months water conditioning (Huang and Söderholm 1989). Although the exact bonding mechanism of phosphate esters to enamel and dentine is not fully understood, it is thought that it is primarily micromechanical in nature. There may be a polar interaction between the negatively charged phosphate groups and positively charged calcium ions in enamel and dentine (Staninec and Holt 1988, Kubo et al 1991).

Since Panavia was introduced by Omura et al (1984), numerous studies showed that it is one of the most clinically successful resin materials to bond to metal. The cement is a modified phosphate ester of Bis-GMA, which provides adhesion to tooth substance, porcelain and various dental alloys. The hydrolysis noted when phosphate ester bonding systems were used on dentine is not usually seen when bonding to metal surfaces.

Kuraray Co Ltd, Osaka, Japan 
The phosphate ester group of monomer is reported to bond directly to metal oxides (Wada 1986). However, blasting dental alloys with alumina results in a significant increase of alumina in their surface compositions (Kern and Thompson 1993a) and 10-MDP containing composite exhibited a high and durable bond strength to glass-infiltrated alumina ceramic (Kern and Thompson 1993b). Therefore, it might be suggested that chemical bonding of 10-MDP containing resin to alumina blasted alloys is also promoted through bonds of 10-MDP to alumina.

Shear bond strengths for Panavia EX to sandblasted Ni/Cr alloy vary from $34.74 \mathrm{MPa}$ (Garcia-Godoy et al 1991) to $17.84 \mathrm{MPa}$ (Souza et al 1994). Difference in the testing conditions between these studies may account for the variation in the shear bond strengths noted. The use of Panavia EX with a sandblasted $\mathrm{Co} / \mathrm{Cr}$ alloy have been reported to give tensile bond strengths of $53.7 \mathrm{MPa}$ (Kern and Thompson 1995).

Panavia does not adhere well to untreated precious alloys or gold, but once these have been tin plated the bond strength is increased (Dixon et al 1994). Micromechanical retention together with chemical bonding, may take place when the gold alloy is tin plated (Matsumura et al 1986, Gates et al 1993). Panavia is shown to be able to maintain its bond strength after water conditioning and thermo-cycling (Thompson et al 1985, Smith et al 1993, Yoshida et al 1996). These results indicated the durability of the bond in vitro, but cannot as yet be directly correlated with clinical service.

Panavia has a unique anaerobic setting reaction. It will not set in contact with air. An $80 \mu \mathrm{m}$ cement film thickness when using Panavia as luting agent, will give the strongest tensile bond strengths (Diaz-Arnold et al 1991). 


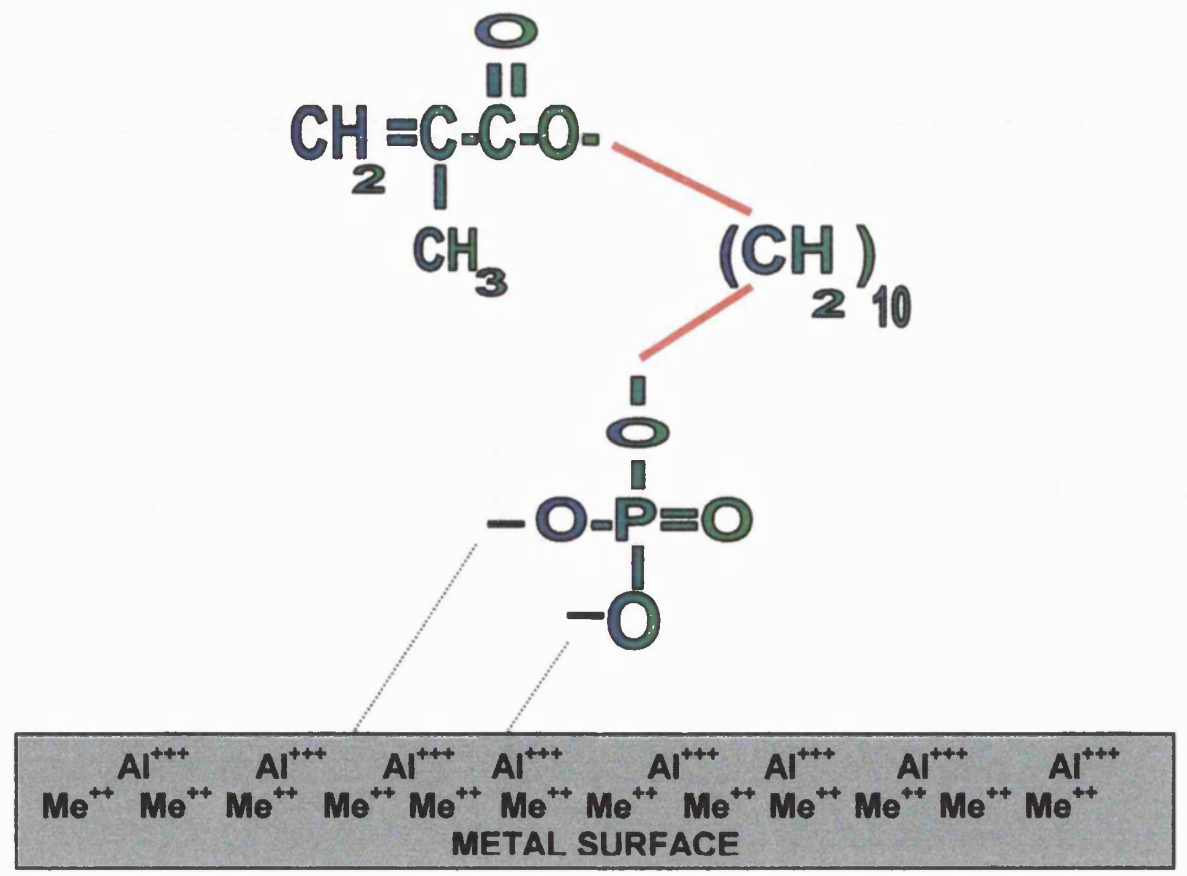

Figure 2.5 : Simplified schematic representation of the 10-methacryloyloxy-decyldihydrogen phosphate (10-MDP) link to the metal surface

Other materials which share the same chemistry to bond to metal include the Clearfil Photo Bond", Cesead opaque primer ${ }^{*}$ and Scotchbond DC ${ }^{* *}$. The Clearfil Photo Bond has been used for metal cementation and repairs (Bertolotti et al 1989, Bertolotti and Paganetti 1990). Scotchbond dual cure primer, without light curing, has provided bonding strengths of up to $30.9 \mathrm{MPa}$ with a sandblasted $\mathrm{Ni} / \mathrm{Cr}$ alloy (Aboush et al 1991). Cesead opaque primer has been shown to be very effective in bonding composite resin to a $\mathrm{Co} / \mathrm{Cr}$ alloy which had been sandblasted with $50 \mu \mathrm{m}$ aluminum oxide. Mean shear bond strength of $44 \mathrm{MPa}$ was reported (Yoshida et al 1993).

Similarly to Panavia, Cesead was resistant to moisture when bonded to non-precious alloys (Yoshida et al 1995 and 1996, Orchard et al 1997, Yoshida et al 1997, Kountouras et al 1999, Yoshida et al 1999).

\footnotetext{
"Kuraray Co Ltd, Osaka, Japan

** 3M Dental Products, St Paul, MN, USA
} 


\subsection{4-META SYSTEMS}

4-META is a synthesised adhesion promoting monomer developed by Tanaka et al (1981), to provide adhesion to non precious alloy and also to precious metals with surface treatments. The material has an affinity for metal oxides. 4-META based systems have shown high bond strengths to non precious alloys with surfaces that have been oxidised (Tanaka et al 1981, Tulunoğlu and Oktemer 1997). Tanaka et al (1986) found that the bond between 4-META and sandblasted $\mathrm{Co} / \mathrm{Cr}$ alloy resisted environmental stresses well. A concern with 4-META products is the early gelation time which occurs long before hardness, due to the dissolution of the polymer powder into the liquid (Uchiyama 1986). This affects their handling properties and may possibly affect their clinical performance.

Aluminum oxide blasting, is routinely being used to create surface oxides. Another method that has also been suggested is ion coating. Ion coating of a base alloy with copper oxide at $400^{\circ} \mathrm{C}$ was found to produce good shear bond strengths, compared with the ones created by aluminum oxide sandblasting, when using 4-META resins (Matsumura et al 1991). However, the disadvantages associated with ion coating is that the technique must be undertaken in the laboratory and is not applicable to denture repairs due to the high temperature involved (Ohno et al 1986). Some commercially available adhesive resins and bonding agents incorporating the 4-META system are, Metadent ${ }^{*}$ (heat cured acrylic resin), Metafast ${ }^{*}$ (cold cured acrylic resin) and Superbond $\mathrm{C}+\mathrm{B}^{*}$. The Super Bond bonding system comprises of a methyl methacrylate resin containing 4META together with a resin monomer. As with Panavia, Superbond bonds strongly to precious alloys and gold after these have been sandblasted and tin plated, or alternatively after they have been heated to over $650^{\circ} \mathrm{C}$. The heating process produces copper oxides

\footnotetext{
'Sun Medical Co Ltd, Kyoto, Japan
} 
on the surface of a type IV gold alloy and it is probably this oxide layer that increases the chemical adhesion to Superbond (Tanaka et al 1988).

Other adhesive resins containing 4-META also include Dynabond" (cold cured acrylic resin), Amalgambond ${ }^{* *}$, Amalgambond Plus ${ }^{* *}$, Cover Up $\mathrm{II}^{* *}$ and Metabond ${ }^{* *}$ bonding systems.

The Amalgambond and Amalgambond Plus systems incorporate a 4-META chemical system as well. The latter system has a new component HPA (high performance additive), which is essentially PMMA powder. This powder enhances the physical properties of the resin adhesive layer and slows polymerisation (Barkmeier et al 1994).

\subsection{BIPHENYLDIMETHACRYLATE SYSTEMS (BPDM)}

Systems containing biphenyl dimethacrylate (BPDM) (Allbond ${ }^{* * *}$, Allbond $2^{* * *}$ ), bond to positively charged metal ions on the alloy surface (Silva e Souza et al 1993). However, unlike 4-META their matrix is Bis-GMA related composite resin, rather than MMA which gives them improved durability.

They bond best to sandblasted metal surfaces. Shear bond strengths to a $\mathrm{Ni} / \mathrm{Cr} / \mathrm{Be}$ alloy after sandblasting are in the range of $21.8 \mathrm{MPa}$ to $27.01 \mathrm{MPa}$ (Barkmeier et al 1991, Kanca 1991, Silva e Souza et al 1993). Unlike phosphate ester based systems, BPDM containing systems do not require tin-plating to precious and semi-precious metal surfaces to obtain high in vitro bond strength (Kanca 1991).

\footnotetext{
"Unitec Corp., Monrovia, Calif., USA

** Parkell Farmingdale,NY, USA

*** Bisco Dental Products, Itasca, Ill., USA
} 


\subsection{PHYSICAL TESTING}

\subsubsection{EVALUATION OF DIFFERENT TESTING CONDITIONS}

During function, an RPD is subjected to a complex stress distribution. Combinations of compressive, tensile and shear stresses develop within the denture components. A shearing stress acts parallel to a plane, as distinguished from tensile and compressive stresses, which act perpendicular to a plane. In an attempt to evaluate the stresses developed within the denture components, various in vitro tests have been developed.

Tension, compression and shear tests usually refer to tests in which a prepared specimen is subjected to a gradually increasing load until failure occurs. These tests attempt to evaluate the mechanical properties of the materials tested, although it must be noted that they produce complex internal stress distributions within a sample, which may be a combination of compression, tension and shear. Thus, they do not provide an unambiguous picture of a material's properties (Darvell 1990).

For brittle dental materials like ceramics the most commonly used tests are the compressive and diametral tensile strength tests. A flexural test is used as an alternative to the other tests by bending a specimen shaped as a beam or disc, inducing compressive stresses over one surface of the specimen and tensile stresses on the opposite side. Therefore a combination of forces are created, and the stress distribution is not uniform across the specimen. It has been suggested that flexural strength testing can be more representative of the forces exerted on a restoration (Yap et al 1994). The main advantage of the flexural tests is that a state of pure tension can be established on one side of the specimen (Berenbaum and Brodie 1959). 
When a force is applied to the occlusal surface or to a clasp of an RPD, flexure of the denture components occurs, exerting tensile, compressive and shear stresses within the materials employed. The components that flex in function are usually supported by a major connector that remains relatively rigid. During uniaxial flexural testing, specimens in the shape of a beam are also rigidly supported and subjected to tensile, compressive and shear stresses.

One important problem in evaluating dental materials is the lack of a standardised test procedure. Devices applicable to fatigue testing are all capable of repeatedly placing a test sample under stress. Some machines attempt to reproduce the intraoral environment by adding moisture and a controlled temperature to the test conditions. However, they may differ considerably in the way they apply stress to the structure compared to each other and in vivo conditions (Wiskott et al 1995). Generally, different magnitudes of stress applied will induce microcracks of different dimensions with a subsequent effect on the stiffness of the material tested. The orientation of the stress applied will determine the orientation of crack development and therefore, depending on the structure of the material tested, will affect its fatigue life.

Generally it is not possible to compare data from one study to another due to the differences in methodology. Review articles by Phillips (1988) and Rueggeberg (1991) discuss numerous variables associated with adhesive testing that have either been shown or are felt to influence the data. These variables may include the test mode e.g. shear, tension or flexural, the design of the test apparatus as it relates to the alignment of specimens, the shape and dimensions of the specimens, the type, mode and point of the load application and the speed of testing. 
The stress at failure between the adhesive and the substrate is calculated as a simple average of the load at failure divided by the cross-sectional area of the bonded surface. Studies of the stress distribution at the adhesive interface for shear bond strength tests, have shown that the stresses at the interface between the adhesive and the substrate are anything but uniform and are highly dependent on the test geometry, loading configuration adopted, size of the adhesive interface and the density of the substrates (van Noort et al 1989, van Noort et al 1991, van Noort 1994, Phrukkanon et al 1998).

In a flexural test there is a difference in the results depending on manner in which the load is applied. A three point bend test will produce different results from a four point test or cantilever loading. The application of load can vary from a single point to an area causing a different dissipation of stresses within the material leading to variable results (Hargreaves 1983).

Finger (1988) concluded in his study that in vitro bond strength figures from different sources are not readily comparable and are only roughly discriminating parameters for the evaluation of an adhesive's efficacy. This does not mean that bond strength tests are of no value. They remain a means of trial for the materials tested. Materials can be ranked during testing by the same source and their properties compared to each other, although absolute values are not necessarily obtained.

Strength and elastic moduli are some of the mechanical properties assessed to evaluate clinical performance (Reeh et al 1989). However, intraoral forces are so complex in nature that none of the common bond strength tests can simulate them accurately (Barkmeier and Cooley 1992). Most failures in the oral environment are due to fatigue failure (McCabe et al 1990). Fatigue is a mode of fracture, whereby a structure eventually 
fails after being repeatedly subjected to loads within the elastic range of the material that one application apparently does nothing detrimental to the component (Nutt 1976). Today fatigue failure is explained by the development of microscopic cracks in areas of stress concentration. With continued loading, these cracks fuse to an ever-growing fissure that insidiously weakens the restoration. Catastrophic failure results from a final loading cycle that exceeds the mechanical capacity of the remaining sound portion of the material. Fatigue failure in polymers can occur by either true mechanical failure or thermal softening or a combination of the two effects.

\subsubsection{FATIGUE TESTING}

\subsubsection{USE OF LOAD CYCLING AS A MEANS OF FATIGUE}

\subsection{MECHANICAL EFFECTS}

It is found that the typical first cycle stress-strain response, at small strain limits, is predominately elastic with associated relaxation/recovery times approximately equal to the cycle time. However, the response to further cycling differs, due to the fact that the compressive stress of polymers is greater than the tensile stress for equal strains. This strength differential is characteristic of the uniform plastic deformation in polymers. It has been proposed that the cyclic softening occurs within the first few cycles and is retained irrespective of time. In composite polymers, this softened state is not truly stable and gradual softening occurs throughout the fatigue life after an initial macroscopic softening (Rabinowitz and Beardmore 1974).

\subsection{THERMAL EFFECTS}

Thermal effects are known to occur commonly in the cyclic testing of polymers and are always likely to be a factor unless special precautions are taken to obviate the effect. The low damping capacity of polymers combines with the low heat capacity and low thermal 
conductivity to promote heating effects. It is relatively easy to induce failure in a polymer by the thermal softening using the appropriate combination of load and frequency. This type of thermal failure is a fatigue failure mechanism directly attributable to the strength loss resulting from an increase in specimen temperature, making the imposed stresses too high to be sustained at this new elevated temperature (Beardmore 1979). On the other hand though, a small increase in temperature may also increase the rate of polymerisation causing an increase in the elastic modulus due to greater conversion (Kandil et al 1989).

Stinchcomb et al (1975) when they examined the effect of the frequency of fatigue loading of composites found that the heat emission depended on the frequency, but that relationship was not linear. Further, real-time surface temperature patterns showed great similarity to observed stress distributions during early testing and subsequently changed, in association with changes in the stress distribution following damage.

\subsubsection{CRACK FORMATION AND PROPAGATION}

Two main interrelated conditions for fracture are proposed. First Griffith's energy criterion (Griffith 1920) proposed that fracture occurs when sufficient energy is stored by the stress field to supply the energy requirements of the new fracture surfaces and thus allows crack growth. Second, the stress field around a sharp crack can be defined by a parameter known as the stress-intensity factor, $K$ (Irwin 1957). This factor defines the magnitude of the local stresses around the crack tip. It depends on loading, crack size, crack shape and geometry of the specimen. Fracture occurs when the value of $K$ exceeds the critical value for crack growth in the material, $K_{C}$ which is often referred to as the "fracture toughness" and is a property of the material. Composite material degradation, in the form of microcrack growth and subsequent crack propagation, is often well established before complete fracture occurs. 
In a composite material, the cracking of the matrix can be delayed by the constraint provided by the filler content. By increasing the filler content, the number of microcracks generated around a main crack is increased and their distribution is also widened, increasing the fracture resistance of the composite (Kim et al 1994). On the other hand though, Lloyd and Iannetta (1982) showed that increasing the filler content over a critical point will make $K_{C}$ decrease leading to a brittle behaviour for the material. It has also been suggested, that cracks may be propagated at low velocities $\left(10^{-7}\right.$ to $\left.10^{-5} \mathrm{~m} / \mathrm{s}\right)$ by the apparent failure of the filler/matrix interfacial bond. Absorbed water affected the strength or fracture resistance of the interface. At high crack velocities the properties of the composite depend on the properties of the polymeric matrix, the filler, and the filler volume fraction, but at low velocities the interface is the controlling factor in the durability of these composites exposed to an aqueous environment (Montes and Draughn 1987).

There is a misconception that there is a direct link between strength and fracture. Although the higher the strength of a material the less likely it is to fracture, this does not mean that high strength materials will not fail nor necessarily will low strength materials invariably fail. Composite veneers and saddles often have quite complex shapes and how these structures will perform under the application of an external load will depend not only on mechanical properties such as strength or stiffness of the materials used, but also on the design of the structure and the amount of load that the structure is expected to carry (Fowler et al 1992, Ritter 1995). 


\subsubsection{USE OF THERMO-CYCLING AND WATER SORPTION AS A MEANS}

\section{OF FATIGUE}

Thermo-cycling is one of the methods for evaluating adhesion durability in metal-bonded resin systems as is long-term water immersion. Thermo-cycling may be regarded as a form of fatigue test. The purpose of thermo-cycling is to induce thermal stressing of materials with different coefficients of thermal expansion. Thermo-cycling can weaken the structure across its thickness and can induce significant amounts of microleakage and fatigue of the bond at the interface of the constituent different materials (Diaz-Arnold and Aquilino 1989, Wictorin et al 1989). Endurance tests determine deterioration of the adhesive interface through a change in bond strength or the degree of dye penetration on exposure of the specimens to a dye solution subsequent to stressing.

The recent progress in the development of metal adhesive systems has considerably improved the durability of adhesion; thus a longer test period than ever is necessary for evaluation. Thermo-cycling has been widely used to simulate intraoral thermal fluctuations, however the reported temperatures used vary considerably and have rarely been substantiated with temperature measurements made in vivo (Gale and Darvell 1999). Nevertheless, with the use of thermo-cycling, testing time can be significantly shortened compared to water immersion alone (Tanaka et al 1995) since it can cause a greater impact on specimens due to increased microleakage. Water diffuses easier through spaces that open up within the material while thermo-cycled (Gale and Darvell 1999).

As described before, different materials have different coefficients of thermal expansion causing stresses to develop at their interface when thermo-cycled. Thus, it is possible that thermo-cycling a composite material can potentially induce stresses at the resin-filler interface causing it to fail. Silanes can initially resist microleakage at the resin filler 
interface, however they eventually hydrolyse since water can attack the Si-O-Si bonds. Similarly, the interface of a composite bonded to a metal restoration, when thermo-cycled will be subjected to similar stresses. The thermal diffusivity and conductivity of metal is higher than that of a resin, causing the temperature change due to thermo-cycling to reach the metal resin interface faster from the metal surface rather than the resin. This difference can influence the stresses set up at the interface.

The temperature chosen as the highest temperature when designing a thermo-cycling endurance test can influence the progress of polymerisation of the adhesive resin, change the material properties if it is near or above glass-transition temperatures and accelerate the water diffusion into the adhesion interface (Tanaka et al 1995). As mentioned before, the temperatures of the thermo-cycling baths reported in the literature vary widely, from $195.8^{\circ} \mathrm{C}$ (Ohno et al 1992 ) to $15^{\circ} \mathrm{C}$ (Peterson et al 1966, Guzman et al 1969) as the solution is cooled and $40^{\circ} \mathrm{C}$ (Ohno et al 1992) to $100^{\circ} \mathrm{C}$ (Hansson 1990) as the solution is warmed. However, cooled water at $4^{\circ} \mathrm{C}-5^{\circ} \mathrm{C}$ and warmed water at a range between $40^{\circ} \mathrm{C}$ and $60^{\circ} \mathrm{C}$ are typical temperatures adopted by most researchers, to simulate the thermal changes to which a restoration would be exposed through the consumption of food (Douglas et al 1989, Momoi et al 1990, Yoshida et al 1990, Staninec and Kawakami 1993). When Gale and Darvell (1999) reviewed various thermo-cycling regimes adopted and reported temperature measurements at the tooth surface in vivo, concluded that while extreme temperatures could conceivably be encountered, they cannot reasonably be taken as typical or representative of the intraoral environment. They suggested that temperatures of $15^{\circ} \mathrm{C}$ and $45^{\circ} \mathrm{C}$ with $35^{\circ} \mathrm{C}$ as the reference resting temperature being clinically relevant. 
When a light cured resin composite is immersed in water two phenomena occur. There is a rapid elution of any unpolymerised monomer or oligomers which appears to be completed within the first few days depending the thickness of the specimens and at the same time diffusion of water inside the resin matrix. Initially, there is no water within the resin material so the surrounding water diffuses inside the resin matrix in an attempt to reach equilibrium. Water swells the polymer in the region of $0.2 \%$ by occupying space between the main chains and cross-links, as well as filling microvoids created during the polymerisation process. Initially the surface of the newly water immersed polymer starts swelling. Owing to an uneven water distribution, that surface swelling will be restrained by the centrally unswollen part. Because of this restraint, the polymer is stressed since its surface will be in compression while the centre in tension (Söderholm 1984b). As time passes diluent resins and monomer components are being released from the resin opening up spaces for more water to diffuse in. Water can diffuse within the polymer mass and break hydrogen bonds between main chain atoms and form their own hydrogen bonds, thereby acting as a plasticiser. It can cause hydrolytic damage to both filler and silane (Papadogianis et al 1985, Beatty et al 1993). The elastic modulus of the matrix is reduced and the debonded fillers may act as stress concentrators, which significantly multiplies the number of potential crack growth sites (Papadogianis et al 1985, Braem et al 1994b).

Depending the thickness of the specimens, equilibrium can be achieved at various time intervals ranging from a few days to months. Ferracane and Condon (1990) for their specimens found that the rapid initial elution could take 7 days to stabilise. Obviously, thicker specimens will take more days for this process to complete. Pearson (1979) suggested that due to the slow nature of diffusion of chemicals into the cross-linked matrix complete saturation of the composite with solvent requires weeks or months to complete. 
This slow uptake of water correlates with the fact that for the majority of the composites, the properties were not reduced significantly until the polymer matrix was saturated with water. As described before, leachable components are removed from the composite during the first few days and water is absorbed to replace them. However once the majority of the elutable components are extracted, water uptake continues to swell and alter the polymer matrix. Hydrolysis of the filler/matrix interfaces and leaching of matrix components as described before, results in softening of the composite (Mante et al 1993) which would contribute to the reduction in its mechanical properties (Ferracane et al 1995, Indrani et al 1995). The colour stability also, can be significantly influenced by chemical corrosion or disintegration of the filler-resin interface and of the resin matrix itself. Leaching of inorganic ions can be detected, during the storage of composites in water. Silicon, boron, barium, strontium and lead which are present in glass fillers, are leached to various degrees $(6-19 \mathrm{mg} / \mathrm{ml})$ from the various resin-filler systems (Söderholm 1981 and 1983, Söderholm et al 1984).

Nevertheless, there is a dispute in the influence of water upon the fracture toughness $\left(\mathrm{K}_{\mathrm{C}}\right)$. Ferracane et al (1995) suggested that water reduces the $K_{c}$ by hydrolysing the bonds between the polymer matrix and inorganic filler particles. On the other hand, Indrani et al (1995) suggested that water uptake causes an increase in the $K_{c}$ by plastising the area in front of the propagating crack tip. Differences in the silane coupling used may account for the difference in the results reported (Indrani et al 1995).

Various chemicals found in the oral cavity can soften dental composites. Wu and McKinney (1982), investigated the softening of the resin matrix on exposure to foodsimulating substances. McKinney and Wu (1985) has described the organic compounds recommended by the Food and Drug Administration (FDA) for simulating foods in vitro. 
Of these compounds water has the highest solubility parameter of $4.8 \times 10^{-4} \mathrm{~J}^{1 / 2} \mathrm{~m}^{-3 / 2}$ and heptane has the lowest solubility parameter of $1.5 \times 10^{-4} \mathrm{~J}^{1 / 2} \mathrm{~m}^{-3 / 2}$. Solubility parameters between these extremes are obtained by mixing ethanol and water (McKinney and $\mathrm{Wu}$ 1985).

Water sorption and thermal changes weaken most metal-resin bonds (Brantley et al 1986). Chang et al (1993), have suggested that, the various bonding systems are influenced to a different extent by the two above parameters. However, Kern and Thompson (1995), found that the metal-resin bond strength in some systems initially increases, while they are stored. This could be explained by a possible optimisation of the chemical bonds taking place during the initial period of bond maturation (Kern and Thompson 1995).

\subsubsection{FLEXURAL TESTING}

Often, the flexural strength and the flexural modulus are evaluated from the three-point flexural test either for certification purposes or as a standard test method for dental composite materials (Vijayaraghavan and Hsiao 1994). While flexural strength can be used as a criterion for design, flexural modulus can predict softening associated with a given in vitro environment but does not provide information on the stress limit at which such form changes are non-recoverable or permanent. Any correlation to observable in vivo deterioration such as marginal deterioration, ditching, or surface microstructural changes associated with wear is also very difficult.

Since the maximum tensile strains are localised on the elongated side of the transverse bend specimen (tensile side), failure is expected to commence at the surface. High local strains can then lead to crack initiation. 
According to Leinfelder et al (1986) and Wassell et al (1992), there is a relationship in between wear and mechanical properties of the material, since wear is often associated with deformation and crack formation just below the surface of the material. However, Harrison and Draughn (1976) suggested that this relationship is not clear and that wear performance is best assessed from long term clinical trials (Peutzfeldt and Asmussen 1992).

\section{$\begin{array}{lllllll}2.5 & \text { EFFECT } & \text { OF LIGHT POLYMERISATION SOURCE AND }\end{array}$ POLYMERISATION SHRINKAGE}

Today visible light sources have replaced old UV sources due to concerns about the safety aspects of the use of high intensity UV light and that the depth of cure that can be achieved with UV light is considerably less than that obtained with visible light (Ruyter and Øysæd 1982b, Leinfelder 1993).

Light activation is accomplished with blue light of about $460 \mathrm{~nm}$, which is absorbed by an initiator, mostly camphoroquinone, causing free radicals to form. Curing lights with high intensity are recommended almost universally. A high light intensity benefits the mechanical properties and water solubility of the composite. Nevertheless, the polymerisation shrinkage also increases with detrimental effects on the marginal adaptation of the material (Lösche et al 1993, Unterbrink and Muessner 1995).

Light-activated resins rely on sufficient intensity of light to achieve adequate polymerisation. As light passes through the composite it is absorbed and scattered, attenuating the intensity, and reducing the effectiveness of the light for resin polymerisation as the depth increases (Rueggeberg et al 1993). Factors affecting the depth of cure may include the filler type, size and content. Hybrids with coarser particles scatter 
the light more than microfines and therefore polymerise more (Ruyter and Øysæd 1982b, Eliades et al 1987, Vargas et al 1998). This view though is open to dispute, since the prepolymerised blocks of microfine composites may often contain filler particles of similar dimensions or surface properties to the ones contained within hybrid ones. Other factors affecting the depth of cure include the thickness and shade of the composite resin with darker shades needing more polymerisation time, the exposure time, the distance from the light source and the light intensity (Cook 1980, Sakaguchi et al 1992). Based on the results of numerous studies, manufacturers currently recommend 40 secs of exposure to a visible light source for adequate polymerisation of resin restorative materials to a 3mm depth (Swartz et al 1983, Atmadja and Bryant 1990).

The final elastic modulus of light curing composites can be influenced by the intensity of the light used for polymerisation (Unterbrink and Muessner 1995, Baharav et al 1997). If a low intensity light is used, it will result in a cured composite with a low elastic modulus. A minimum output intensity of light of $280-300 \mathrm{~mW} \mathrm{~cm}^{-2}$ has been recommended (Shortall and Harrington 1996).

The resin component of the composites shrinks with polymerisation and large compressive stresses at the resin-filler interface in a radial direction and tensile stresses in the matrix oriented in a direction parallel to the filler interface can be induced (Söderholm 1984a). These stresses, especially when combined with stress-raising factors such as sharp filler edges, will facilitate crack growth and filler debonding. However, these stresses got reduced when the filler particles were not silane treated (Condon and Ferracane 1998). When used for veneering, polymerisation shrinkage may potentially result in a set composite that is not well adapted to a metal substrate since there could be 
contraction away from the metal during light curing depending the dimensions and configuration of the metal substrate surface.

\subsection{CURRENT STATUS OF COMPOSITE MATERIALS}

Composite materials have advanced to the point that they are highly filled and also have variable levels of modulus of elasticity. The filler/resin interface has been enhanced substantially too. Adhesive resins have been developed that bond composite resin materials to metal. It would therefore seem to be a good point to assess the use of two different types of reinforced resin as a means of aesthetic coverage of a removable partial overlay denture. The determination of water sorption and solubility after water conditioning and also stress at failure and flexural modulus after fatigue conditioning, help characterise these materials and also relate them to clinical performance. 


\section{STATEMENT OF THE PROBLEM}

\subsection{THE PROBLEM}

Composite resins may be used for different components of the RPD to satisfy the aesthetic needs of the patient. Often, composite resins need to be in contact with a metal alloy substructure. Not only should the composite resin be retained to the alloy initially, but also during the life of the prosthesis in function. When employed on occlusal surfaces, the composite resin is subjected to wear and little flexure, while on a clasp flexural forces dominate. During mastication of food, the components of the prosthesis are subjected to both thermal and loading stresses.

The elasticity of the constituent polymer and metal alloy materials of the prosthesis after simulated ageing has been examined in the past through in vitro shear, tensile and flexural tests. However, further in vitro investigations are needed, to clarify the effects that cyclical loading and thermal cycling have on the elasticity of components made of different composite resin materials bonded to a metal substructure, similar to the way they are employed in vivo.

\subsection{HYPOTHESIS}

The flexural modulus of a laminate made of a microfine composite bonded to a metal substructure will be less than that of a hybrid composite bonded to metal after being cyclically loaded and thermally stressed. 


\section{MATERIALS AND METHODS}

\subsection{EQUIPMENT AND MATERIALS USED}

Where possible, commercially available items of equipment were used in the preparation and testing of the experimental specimens, though, some items of equipment were of necessity purpose built.

The equipment used are listed in Appendix II.

The materials used are listed in Appendix III.

\subsubsection{THE CASTING ALLOY}

The casting alloy had a composition of $61 \%$ cobalt, $29 \%$ chromium and $5 \%$ molybdenum. The remaining $5 \%$ of the alloy contains manganese, silicon and carbon. This alloy melts at $1380^{\circ} \mathrm{C}$ and solidifies at $1200^{\circ} \mathrm{C}$.

\subsubsection{THE COMPOSITE RESINS}

The following composite resins were used in this study:

\subsubsection{SILUX PLUS}

Silux Plus is a visible light activated, microfill composite resin. It contains $40 \%$ by volume, $56 \%$ by weight, colloidal silica with an average particle size of $0.04 \mu \mathrm{m}$. The particle size ranges from 0.04 to $0.09 \mu \mathrm{m}$. The resin matrix contains bis-glycidyl methacrylate (Bis-GMA) and triethylene glycol dimethacrylate (TEGDMA) resins (Silux Plus technical profile). It contains prepolymerised heat cured resin fillers in an attempt to reduce the amount of unpolymerised resin in the cured composite. The mean particle size of these ground, prepolymerised particles is $22 \mu \mathrm{m}$ (Willems et al 1993). The manufacturing process involves the incorporation of fine colloidal silica into the resin in 
which a volatile solvent is added to decrease its viscosity. The solvent is evaporated and the resin is cured to produce a polymer block with filler incorporated into it. This block is then ground down and the fragments incorporated into the final resin. The incorporation of these prepolymerised particles reduces the polymerisation shrinkage. The surface is polymer rich leading to increased polishability. The material used was disposed in syringes.

\subsubsection{Z100}

$\mathrm{Z} 100$ is a visible-light activated, radiopaque, restorative composite resin. It is designed for use in both anterior and posterior regions of the mouth. The filler is zirconium/ $/ \mathrm{SiO}_{2}$. It is produced by a process in which precursors of the glasses, such as metal carboxylates and metal oxides, are mixed in a volatile solvent and then dehydrated to form filler particles. This is known as a sol/gel process. The fillers are ground in such a way to produce round particles (Ferracane 1995). The inorganic filler loading is $66 \%$ by volume, $84.5 \%$ by weight, with a particle size range of 3.5 to $0.01 \mu \mathrm{m}$. The resin matrix contains bis-glycidyl methacrylate (Bis-GMA) formulations which contain pendant hydroxyl groups on the backbone chain and triethylene glycol dimethacrylate (TEGDMA) resins as a diluent (Z100 product information). These pendant hydroxyl groups may influence the water sorption behaviour of the material as water can react with them. The material used was disposed in syringes.

\subsubsection{CESEAD}

Cesead is a three element composite resin system. It has an opaque primer, a body opaque resin, and a hybrid composite veneering resin. The opaque primer is an insoluble liquid with a specific gravity of 0.8 . It contains bisphenol A polyethoxy dimethacrylate (5-10\%), hydrophobic dimethacrylate (1-5\%), a metal-adhesive phosphate monomer 10 - 
methacryloyloxydecyl dihydrogen phosphate (10-MDP) $(0-1 \%)$ that bonds to the alloy surface, tert-butyl alcohol (60-100\%), methyl isobutyl ketone (10-30\%) and benzoyl peroxide $(0-1 \%)$. The body opaque resin is an insoluble coloured paste that bonds to the primer. It is a dual cured system, which is light activated. It will additionally cure chemically when in contact with the opaque primer. This allows polymerisation to proceed in the event that light is unable to penetrate through the entire thickness of the resin. It contains silanated silica $(10-30 \%)$, silanated titanium oxide $(5-10 \%)$, barium sulphate (5-10\%), a prepolymerised organic filler containing colloidal silica (10-30\%), bisphenol A polyethoxy dimethacrylate (30-60\%), bisphenol A diglycidilmethacrylate (5$10 \%)$, colloidal silica (1-5\%), DL-camphorquinone (0-1\%), N,N-diethanol-p-toluidine (01\%). The body opaque has an elastic modulus of $5.4 \mathrm{GPa}$ (Cesead product information).

A veneering resin is also provided. This is a light-cured hybrid composite with a filler content of $82 \%$ by weight. It contains silanated glass together with prepolymerised organic filler in its composition. Its resin matrix contains bisphenol A diglycidilmethacrylate, triethyleneglycol dimethacrylate, urethane tetramethacrylate (Cesead product information). It seems that the filler particles in this composite are a mixture of fillers commonly found in microfine composites and others that are commonly found in hybrids. For the purpose of this study only, the opaque primer and body opaque resin were used. 


\subsection{METHODS}

A preliminary study was undertaken, to assess the mechanical properties of the components of the specimens and also to evaluate the sorption behaviour of the resins when immersed in water.

\subsubsection{METHOD TO ASSESS THE MECHANICAL PROPERTIES OF THE COMPONENTS}

\subsubsection{MANUFACTURE OF THE METAL COMPONENT OF THE LAMINATE SPECIMENS}

Casting wax sheets were placed over graph paper and cut freehand using a scalpel with the aid of a metal ruler, to produce strips of wax $0.8 \mathrm{~mm}$ thick, $4 \mathrm{~mm}$ wide and $30 \mathrm{~mm}$ long. The cut wax patterns were attached to a warm investment base, so that they could stick firmly to it. They were invested in groups of five per investment ring, joined by a central sprue. The same diameter sprue wax was used for all the castings. Four ingots of $\mathrm{Co} / \mathrm{Cr}$ alloy were used per investment ring. The casting machine lacked the capacity to produce all the specimens at one time. To minimise procedure variation, each investment mould was placed in a furnace and held at $1000^{\circ} \mathrm{C}$ for 30 minutes before casting, according to the manufacturers instructions. The alloy was heated by an electrical induction element and the specimens cast by centrifugal force, as per the manufacturer's instructions. The advice to cool in air for 10 minutes after casting, then immerse in cold water, was followed closely. The castings were removed from the sprue, cleaned and deinvested using a mixture of coarse and extra-coarse sandblasting grit (Blast grain ${ }^{*}$ ). The abrasive particles were applied as long as it was necessary, to remove the investment residue. The castings were then trimmed to produce metal spines of similar dimensions to the prepared wax.

\footnotetext{
"Chapelin \& Jacobs LTD, Surrey, England
} 
After manufacture, the width and thickness of each metal component of the laminate specimens was measured using a digital micrometer at 4 sites along the specimen length. The mean value for each dimension was entered into a computer test programme controlling an Instron $4505^{*}$ test machine. Each metal specimen was mounted on the jig shown in Figures $4.14 a+b$. The load was applied in the centre of the metal specimen. The jig was placed into the testing machine and the crosshead moved until the load display indicated that minimum force of $0.5 \mathrm{~N}$ was applied to the specimen. The displacement of the crosshead was set at zero and a three point bend test was performed on each specimen up to a point of plastic deformation at a crosshead speed of $2 \mathrm{~mm} / \mathrm{min}$. A maximum extension of $10 \mathrm{~mm}$ was set at the computer program to mark the end of force application if fracture had not occurred previously. $10 \mathrm{~mm}$ limit of the extension was enough to ensure that the specimen would be subjected to plastic deformation by the end of the three point bend test, since trial metal specimens tested earlier, deformed plastically at a shorter extension than $10 \mathrm{~mm}$. The elastic modulus and stress at failure were obtained for each specimen via the computer's controlled printer and plotter linked to the universal load testing machine.

\subsubsection{MANUFACTURE OF THE RESIN SPINE SPECIMENS}

In addition to the preparation and testing of the metal component of the laminate, 10 spines made of $Z 100$ and 10 spines made of Silux Plus composite resins $(22 \times 4 \times 2 \mathrm{~mm})$ were prepared using the specimen mould shown in Figures $4.7 \mathrm{a}+\mathrm{b}$. In order for the resin spines of the above dimensions to be manufactured, a modification had to be carried out to the specimen mould. A solid brass plate $0.8 \mathrm{~mm}$ thick, without any openings, had to substitute the $0.8 \mathrm{~mm}$ thick silicon sheet with the 10 openings of $4 \times 30 \mathrm{~mm}$ shown in Figures $4.8 \mathrm{a}+\mathrm{b}$. The composite resin was applied in a single $2 \mathrm{~mm}$ thick layer and light

\footnotetext{
"Instron Corp., 100 Royal St.,Canton, Mass 02021, USA.
} 
cured initially with the visible light curing unit (Luxor ${ }^{*}$ ) for 40 seconds. The specimens were then removed from the mould and placed in the light curing vessel (Unilux $\mathrm{AC}^{* *}$ ) for a further 180 seconds. The light curing vessel is shown in Figure 4.1.

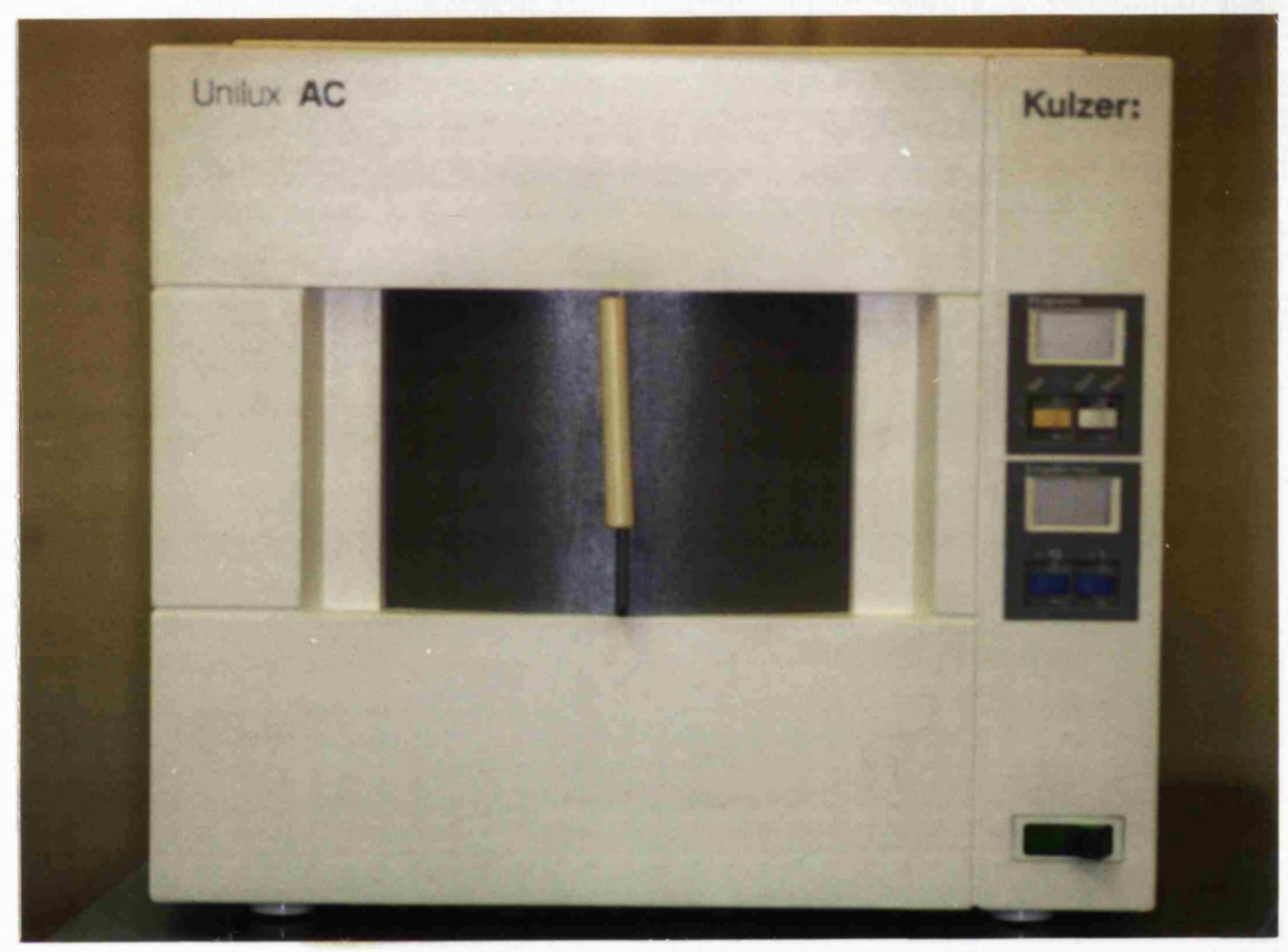

Figure 4.1 : The Kulzer Unilux AC light curing vessel

Two curing units were used in an attempt to ensure the maximum degree of conversion of the monomer in the specimens. Although the thickness of the composite resin spine of the specimens was only $2 \mathrm{~mm}$, the specimen mould did not allow all-around polymerisation of the resin since only the top surface of the composite resin spine could have been polymerised initially by the hand held light curing unit. The placement of each specimen in the light curing vessel ensured all-around polymerisation.

\footnotetext{
*ICI Pharmaceuticals Division, Macclesfield, England

** Kulzer GMBH,Bereich Dental, Phillip-Reiss, Germany.
} 
These specimens were loaded to failure immediately after manufacture in a three point bend test as used for the metal components of the laminate specimens. A diagrammatic representation of the jig used is shown in Figure 4.2. The same method of testing used for the metal components was also used for the testing of the composite resin spine specimens. Loading rate was maintained at $2 \mathrm{~mm} / \mathrm{min}$. The elastic modulus and stress at failure were obtained for each specimen via the computer controlled printer and plotter linked to the universal load testing machine. The point of failure was determined by the computer program. When the load decreased abruptly at any point during testing the computer program regarded it as failure, fracture having already occurred.

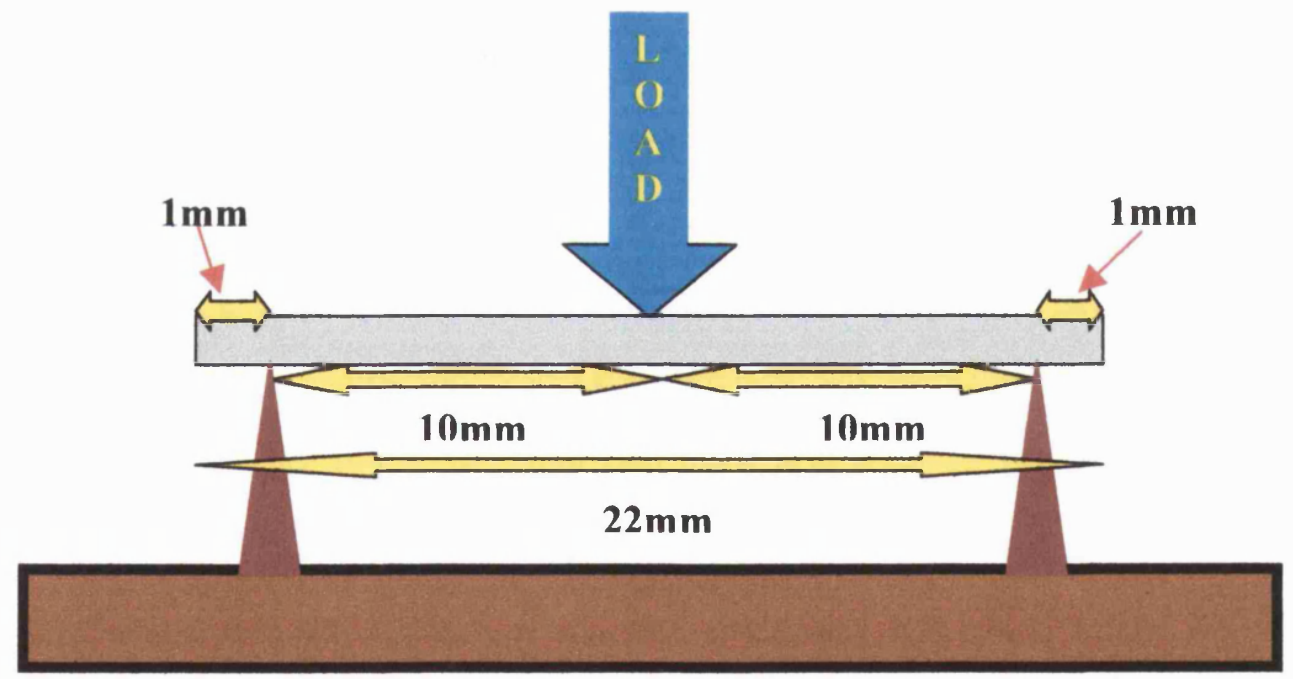

Figure 4.2 : Dimensions of the jig used to test the composite resin spine specimens 


\subsubsection{METHOD TO EVALUATE THE SORPTION BEHAVIOUR OF THE RESINS}

A study of the sorption of water by the two composite resins was undertaken. A perspex mould was manufactured to permit composite resin discs of dimensions of $10 \mathrm{~mm}$ diameter and $2 \mathrm{~mm}$ thickness to be prepared. The thickness was determined to be similar to that of the resin spines used in the mechanical component of the study.

10 disc specimens of Z100 and 10 disc specimens of Silux Plus were prepared by packing the composite into the mould and initially curing for 40 secs using the visible light curing unit (Luxor). Subsequently the discs were placed in the light curing vessel (Kulzer) for a further 180 seconds as described previously.

The specimen mould is illustrated in Figure 4.3a and its dimensions shown in Figure 4.3b. A typical specimen is shown in Figure 4.4a and its dimensions are shown in Figure 4.4b. 


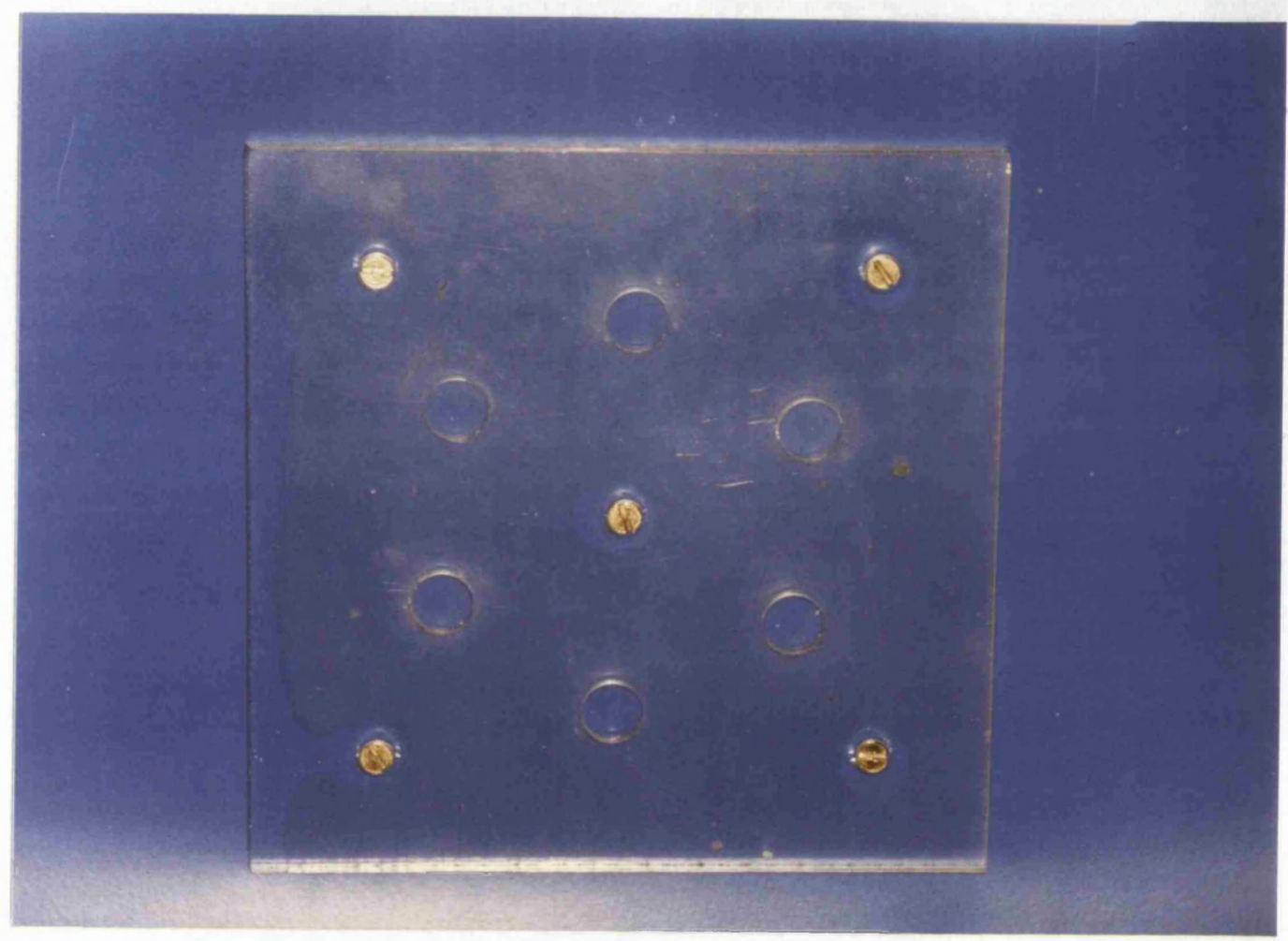

Figure 4.3a : The specimen mould for the water sorption study

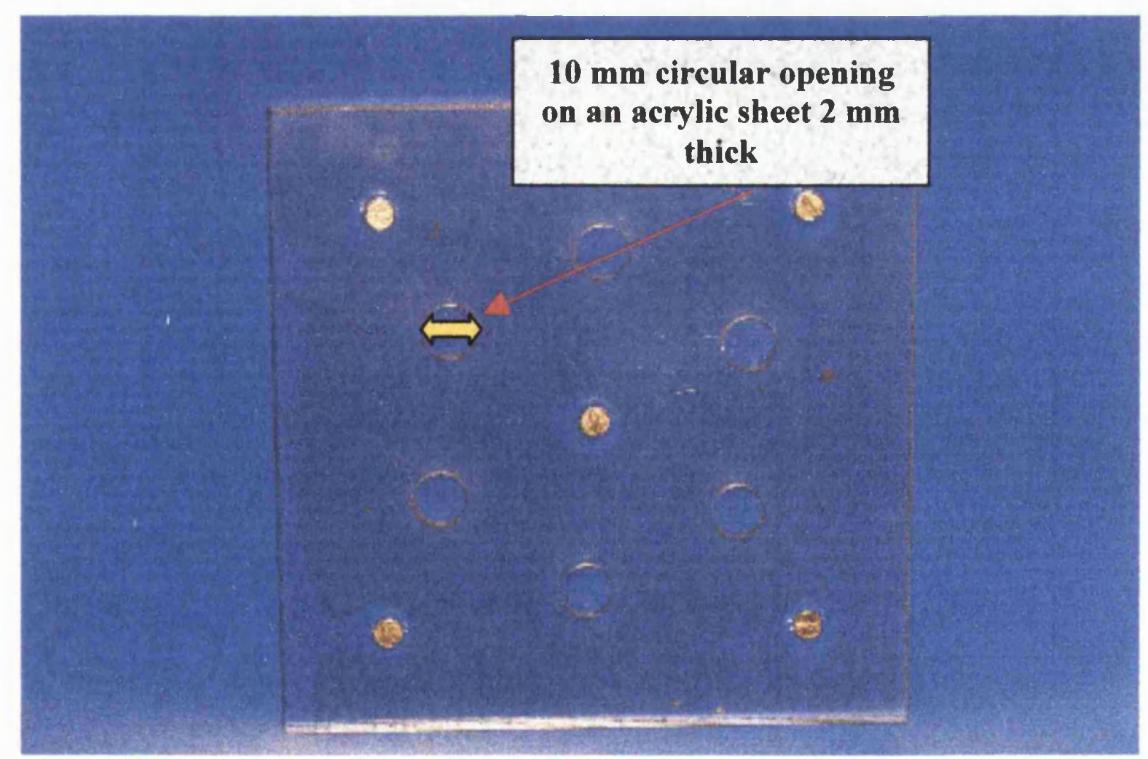

Figure 4.3b : The dimensions of the specimen mould for the water sorption study 


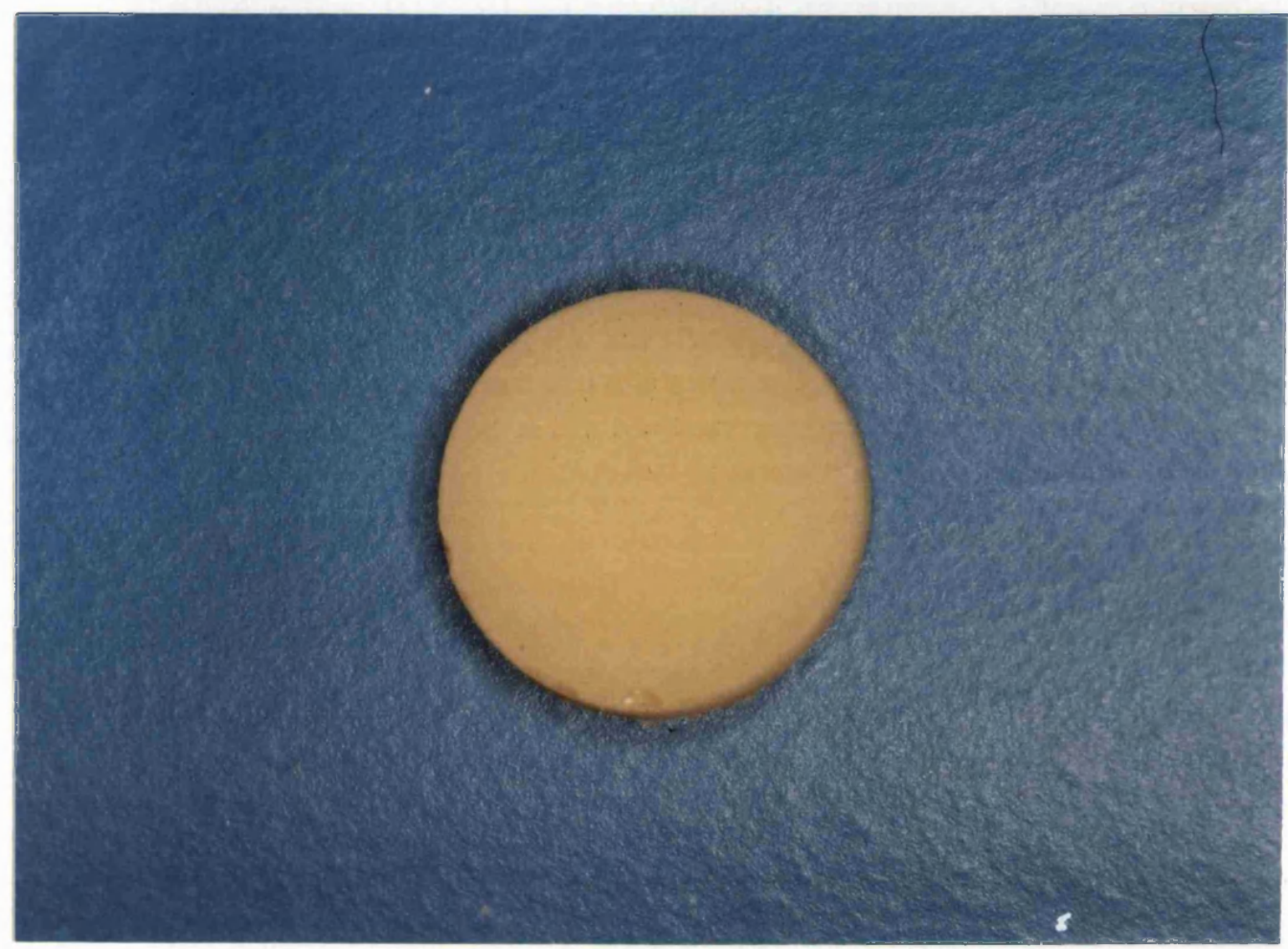

Figure 4.4a : A typical specimen for the water sorption study

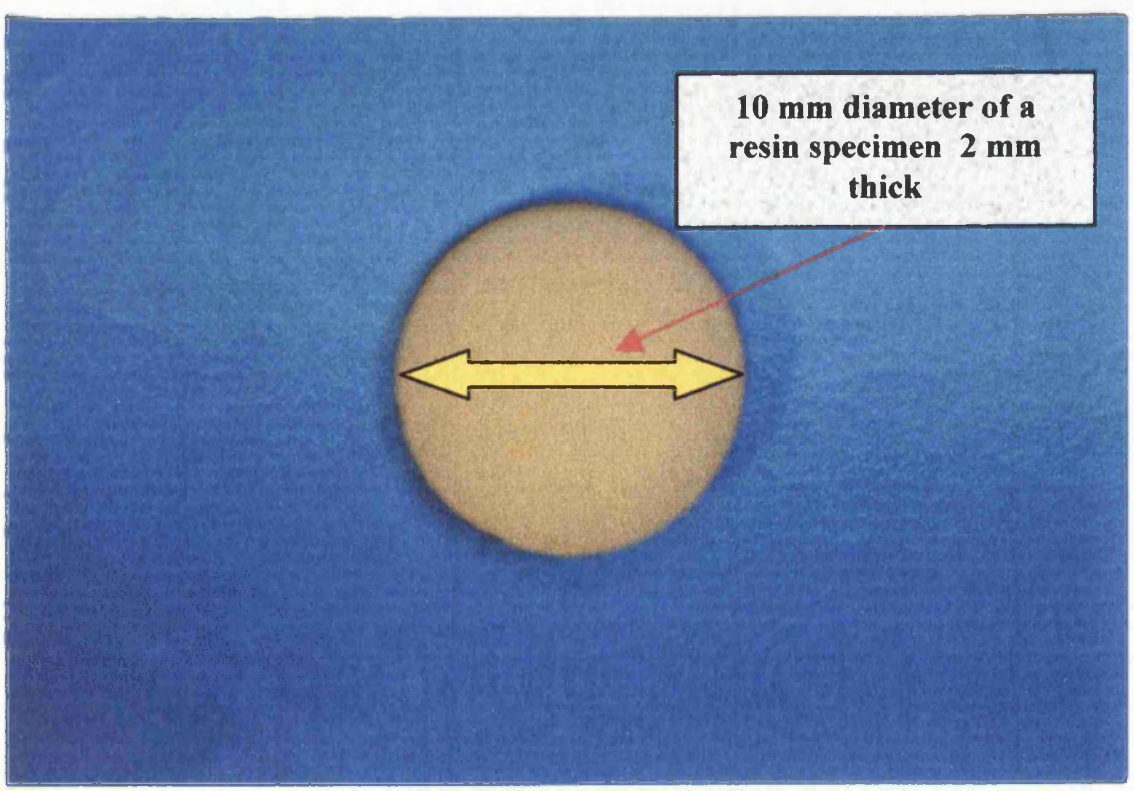

Figure 4.4b : The dimensions of a typical specimen for the water sorption study 


\subsection{MEASUREMENT OF VARIATION IN WEIGHING}

In order to determine the experimental variation which occurred during weighing, due to temperature and environmental changes around the balance, a metal surgical razor blade was weighed at regular intervals over a period of three days. In between measurements the blade was kept inside a desiccator containing silica gel to minimise the effects of humidity upon the metal.

\subsection{FIRST SORPTION CYCLE}

All the specimens were weighed to $\pm 0.0001 \mathrm{~g}$, using an analytical balance (PRECISA ${ }^{*}$ ). They were then placed in a desiccator containing silica gel, where they were retained until a constant mass was achieved. Then the discs were placed in $100 \mathrm{ml}$ of deionized water at $37^{\circ} \mathrm{C} \pm 1{ }^{\circ} \mathrm{C}$. The specimens were then reweighed in regular time intervals of $1,3,6,24$, $48 \& 72$ hours, $7 \& 14$ days, and at two week intervals thereafter until a constant mass was achieved. Each weighing was performed by removing the specimen from the water with tweezers, and blot drying it until free from visible moisture for a time less than 15 seconds. The specimen was then held in air for 5 seconds, prior to placement on the balance. All weighing was carried out within one minute of removal from the water.

Although the mass of each specimen was monitored for 12 weeks, after a period of 6-8 weeks, equilibrium appeared to have been achieved since mass changes were very small after this time. After 8 weeks the changes in mass were very similar to the experimental variation of the weighing procedure. The specimens were finally weighed in the end of the 12 week period and then replaced in the desiccator. They were then reweighed at various intervals until a constant desiccated mass was obtained.

\footnotetext{
- PAG Oerlicon, Zurich, Switzerland
} 
From the values obtained, the percentage of water sorption was calculated as follows:

Mass after Immersion - Conditioned Mass at the start of the $1^{\text {st }}$ cycle

Conditioned Mass at the start of the $1^{\text {st }}$ cycle

The mass gain for each of the specimens was plotted against the $t^{1 / 2}$. A scatter diagram of the mass values was also obtained at the time intervals specified.

\subsection{SECOND SORPTION CYCLE}

The specimens were then reimmersed in deionized water and the sorption cycle repeated. All weighing was performed at the same regular intervals as in the first sorption cycle up to week 12. Although equilibrium appeared to have been achieved for the second cycle after a period of 4-6 weeks, the specimens were kept immersed for 12 weeks similar to the first cycle. At the end of 12 weeks the mass of each specimen was noted and the specimens were again placed in the desiccator and weighed at regular intervals until a constant mass was maintained. At that point desiccation was considered complete. The specimen mass was then recorded and compared with the mass at the start of the second sorption cycle. The $\mathrm{pH}$ of the storage water was also monitored during the test period using a pH electrode (Orion 901 Ionalyser").

\footnotetext{
"Quadrachem Labs, East Sussex, England
} 
From the values obtained, the percentage of water sorption was calculated as follows:

Mass after Immersion - Conditioned Mass at the start of the $2^{\text {nd }}$ cycle x 100

Conditioned Mass at the start of the $2^{\text {nd }}$ cycle

The mass gain for each of the specimens was plotted against the $t^{1 / 2}$.

The solubility for both composites tested was also calculated from the following formula:

Conditioned Mass at the start of the $1^{\text {st }}$ cycle - Mass at the end of the $1^{\text {st }}$ cycle

Conditioned Mass at the start of the $1^{\text {st }}$ cycle

A scatter diagram of the mass values was also obtained at the time intervals specified.

\subsubsection{MAIN THERMAL AND FLEXURAL LOADING STUDY}

\subsubsection{SPECIMEN PREPARATION FOR THE MAIN THERMAL AND}

\section{FLEXURAL LOADING STUDY}

\subsection{PREPARATION OF THE METAL COMPONENT}

600 castings were prepared in a manner similar to the preliminary study. The surfaces of the castings were dried with oil-free compressed air and their dimensions were verified as before. Each casting was placed in a plastic jig, designed to permit the nozzle of an air abrasive gun (Sandstorm ${ }^{*}$ ) to be positioned $10 \mathrm{~mm}$ from the casting surface. This surface was then air abraded with $50 \mu \mathrm{m}$ aluminum oxide $\left(\right.$ Strahlmittel $\left.^{* *}\right)$ at $80 \mathrm{psi}$ for 30 seconds. The jig used to keep a constant distance in between the blaster tip and the metal specimen

\footnotetext{
"Chapelin and Jacobs Ltd, Surrey, England

${ }^{\text {"* }}$ Renfert GmbH, Hilzingen, Germany
} 
is drawn in Figure 4.5. The nozzle itself was moved freehand trying to keep a uniform speed of the jet as it passed along the specimen surface. The aim was to pass the jet three times over the entire length of the metal surface during the blasting period. Considering the length of the metal surface $(30 \mathrm{~mm})$, the times that the surface was blasted $(3 x)$ and the time of blasting (30secs) a speed of passage of the jet of approximately $3 \mathrm{~mm} / \mathrm{sec}$ was

calculated. The specimens were then cleaned ultrasonically in distilled water for 5 minutes and dried with oil-free compressed air.

\subsection{PREPARATION OF THE RESIN COMPONENT}

The following combinations of resin systems for application to the metalwork were prepared:

i) Z100 composite/CESEAD opaque primer and resin

ii) Silux Plus composite/CESEAD opaque primer and resin

The procedures undertaken to bond the resin on the metal surface are shown in Figure 4.6. 


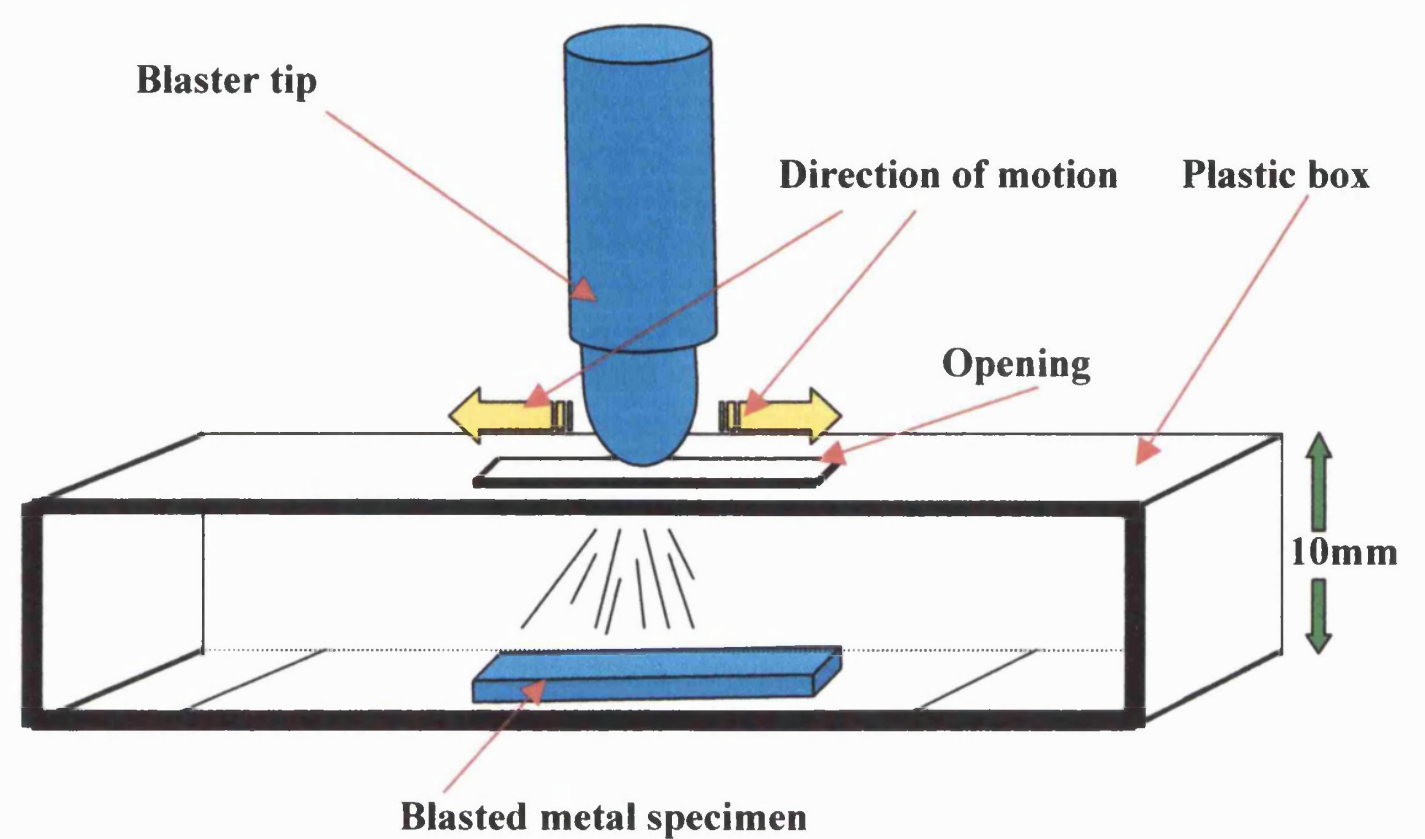

Figure 4.5 : Diagram of the jig used to abrade the metal alloy specimens

Following the preparation of the metal surface as described above, opaque CESEAD primer was applied to the tip of the applicator brush and then brushed thinly onto the metal surface with two brush strokes according to the manufacturer's instructions. To ensure a similar quality of the blasted surface in between castings, all metal specimens were monitored for a similar "light gray" colour of the blasted surface before primer application and the "spontaneous" spread of the primer on the surface when applied. The volatile organic solvents were evaporated by application of a gentle stream of oil-free compressed air for 3 seconds at a distance of $5 \mathrm{~cm}$. CESEAD opaque resin was brushed on in a thin layer according to the manufacturer's instructions and light cured for 180 seconds in the Kulzer light curing vessel. The thickness of the opaque layer was not measured for individual specimens. A standardised technique was used to deliver similar amounts of the material on the surface of the metal component. A similar small amount of 


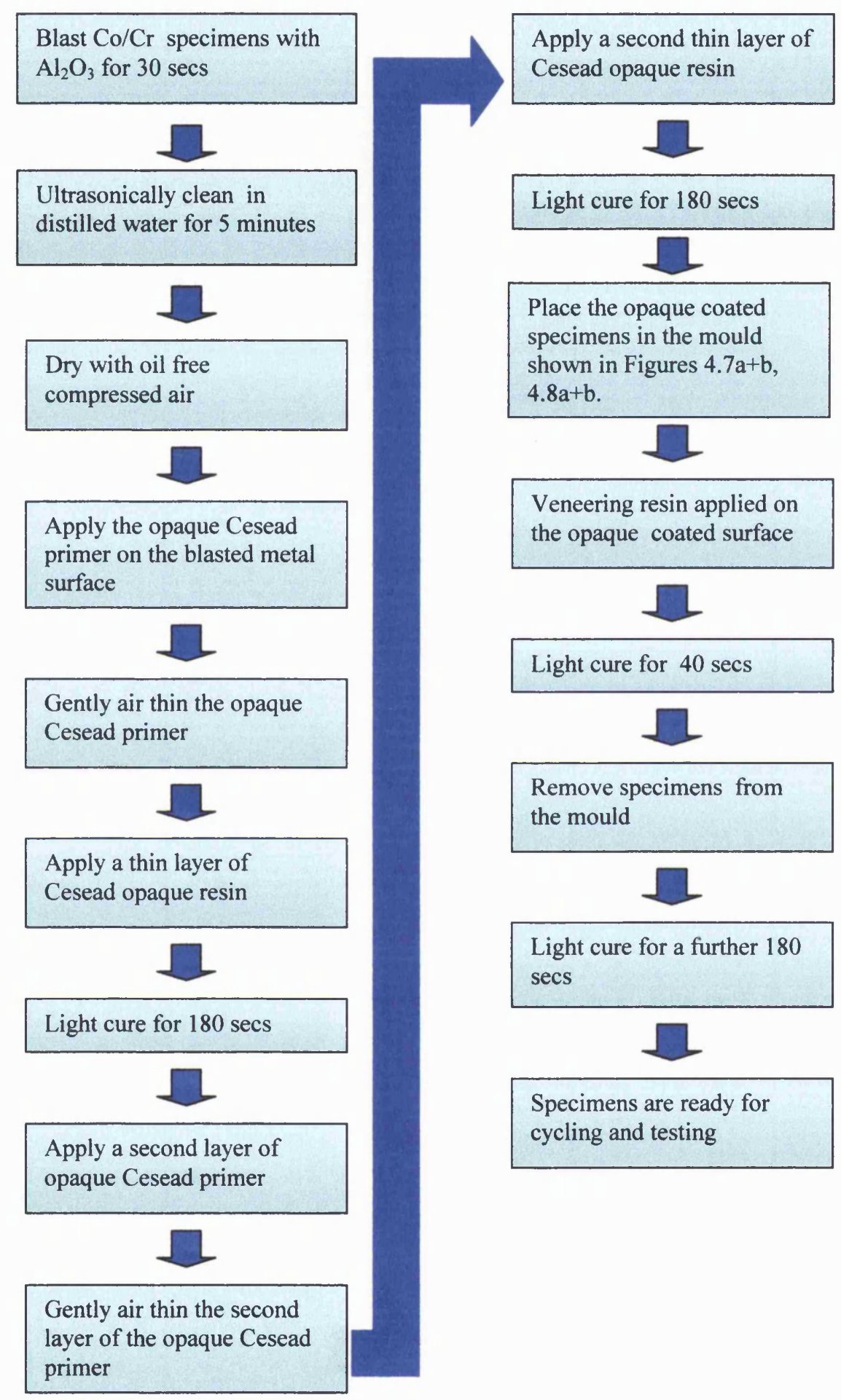

Figure 4.6 : Flow diagram of the procedures undertaken to manufacture the resin bonded to metal laminates 
opaque was dispensed from the syringe and applied with two single brush strokes along the length of the specimen. The manufacturer's instructions for application were followed closely during the procedure.

A second coat of primer was then applied and dried as for the first layer. This was followed by a second opaque resin also applied and cured in like manner to the first. The size of the chamber of the Kulzer light curing vessel allowed for the ten specimens of each of the groups to be cured together. The CESEAD coated specimens were then placed in the mould shown in Figures $4.7 \mathrm{a}+\mathrm{b}, 4.8 \mathrm{a}+\mathrm{b}$. This mould could accommodate 10 castings at any one time, and was sized to produce a resin spine of $22 \times 4 \times 2 \mathrm{~mm}$ on each prepared casting. The layers of material overlaying each casting are shown in Figure 4.10.

The resin was applied in a single $2 \mathrm{~mm}$ thick layer and light cured initially with the hand held light curing unit (Luxor) for 40 seconds. The specimens were then removed from the mould and placed in the light curing vessel (Kulzer) for a further 180 seconds. A typical specimen is illustrated in Figures $4.9 \mathrm{a}+\mathrm{b}$.

\subsection{FINISHED SPECIMEN HANDLING AND CREATION OF THE TESTING GROUPS}

The long edges of the bonded resin to metal specimens were trimmed using 320 grit silicon carbide abrasive paper discs used wet on a flatbed rotary abrader in order to trim any excess composite or opaque resin present in the side of the specimens. The specimens were then divided into different groups for further testing as seen in Figures 4.11 and 4.12. The 600 cast specimens, were split into two groups. 300 resin spine specimens were manufactured using Silux Plus as the veneering resin, and 300 using Z100. 


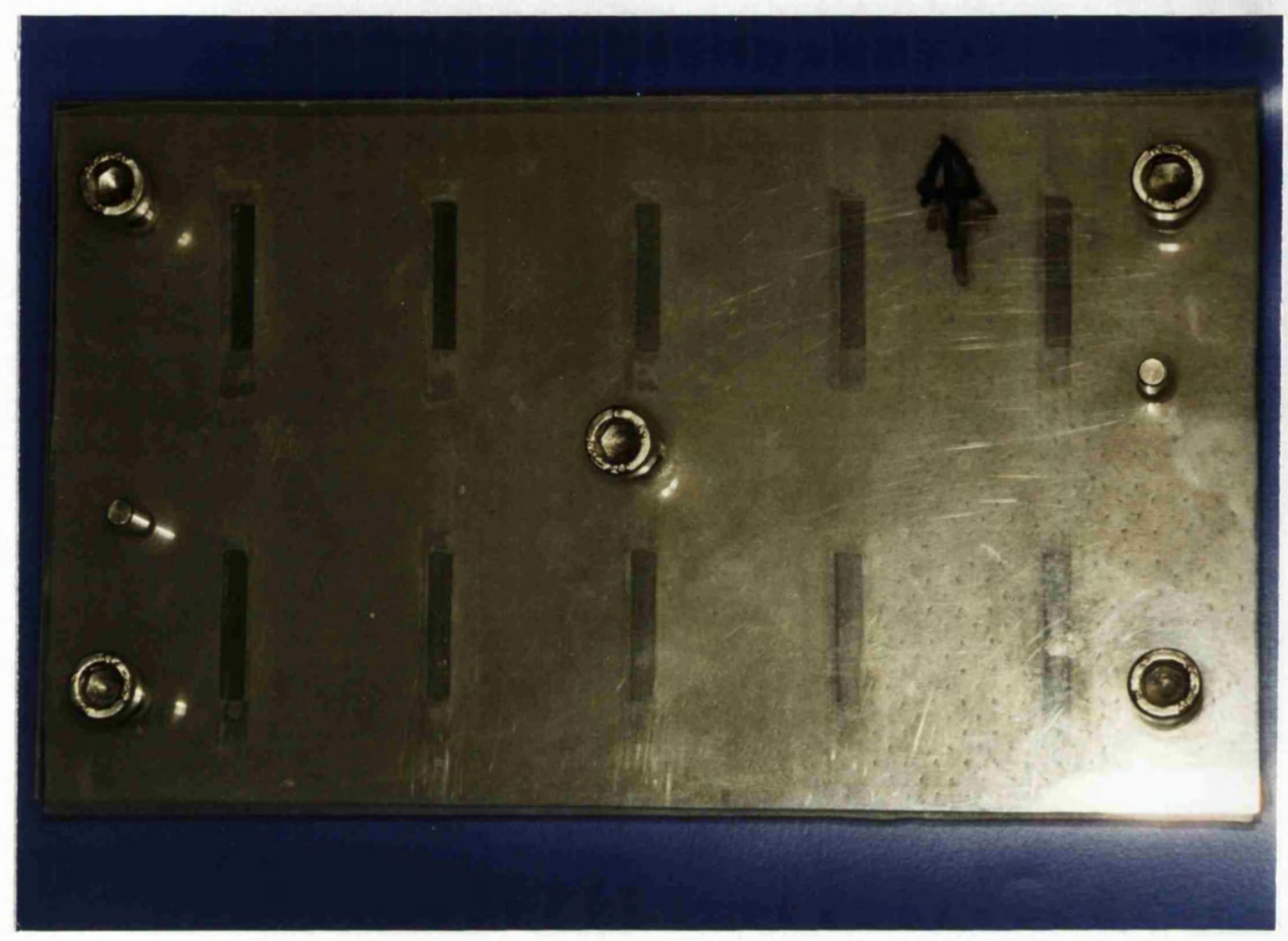

Figure 4.7a: The specimen mould used to manufacture the resin to metal bonded specimens

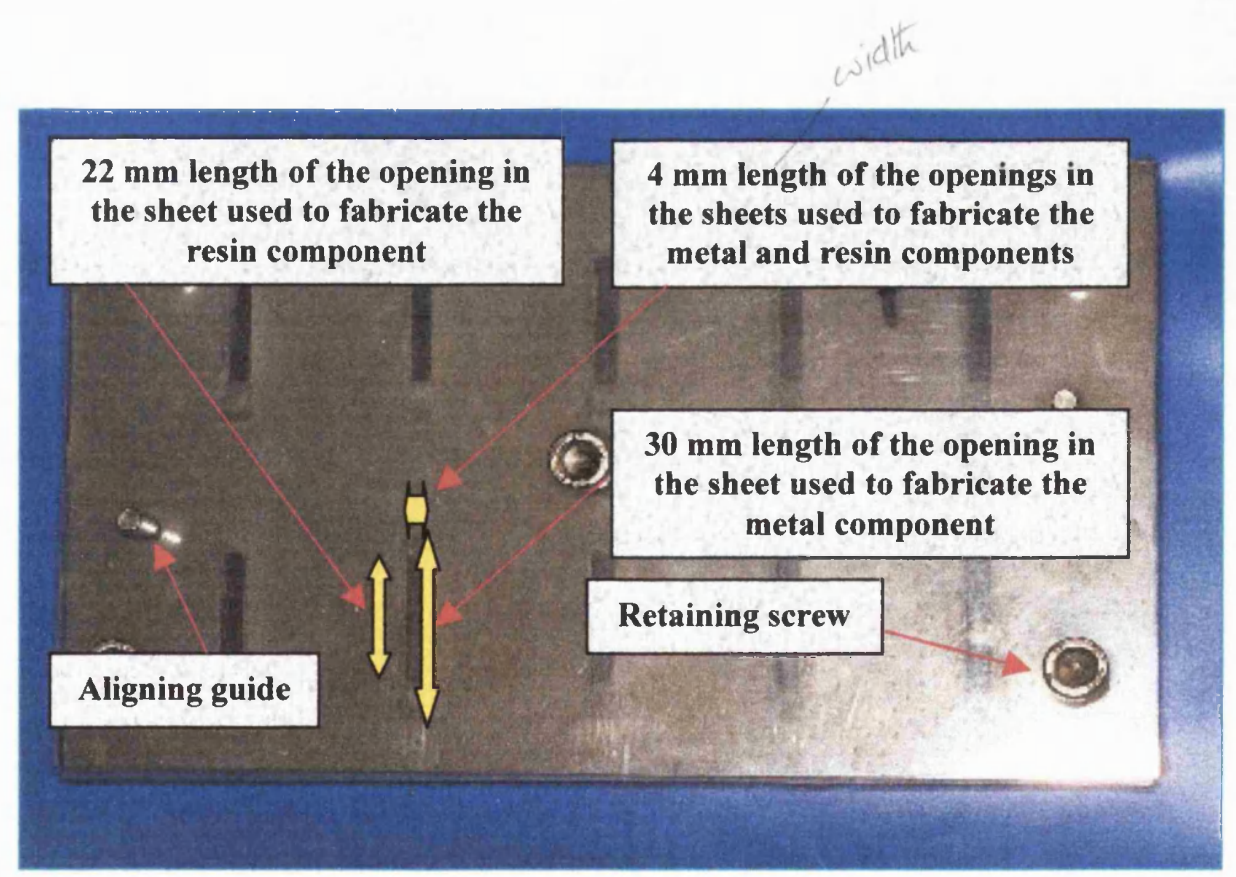

Figure 4.7b : A representation of the different lengths of the various components of the specimen mould used to fabricate the bonded resin to metal specimens 


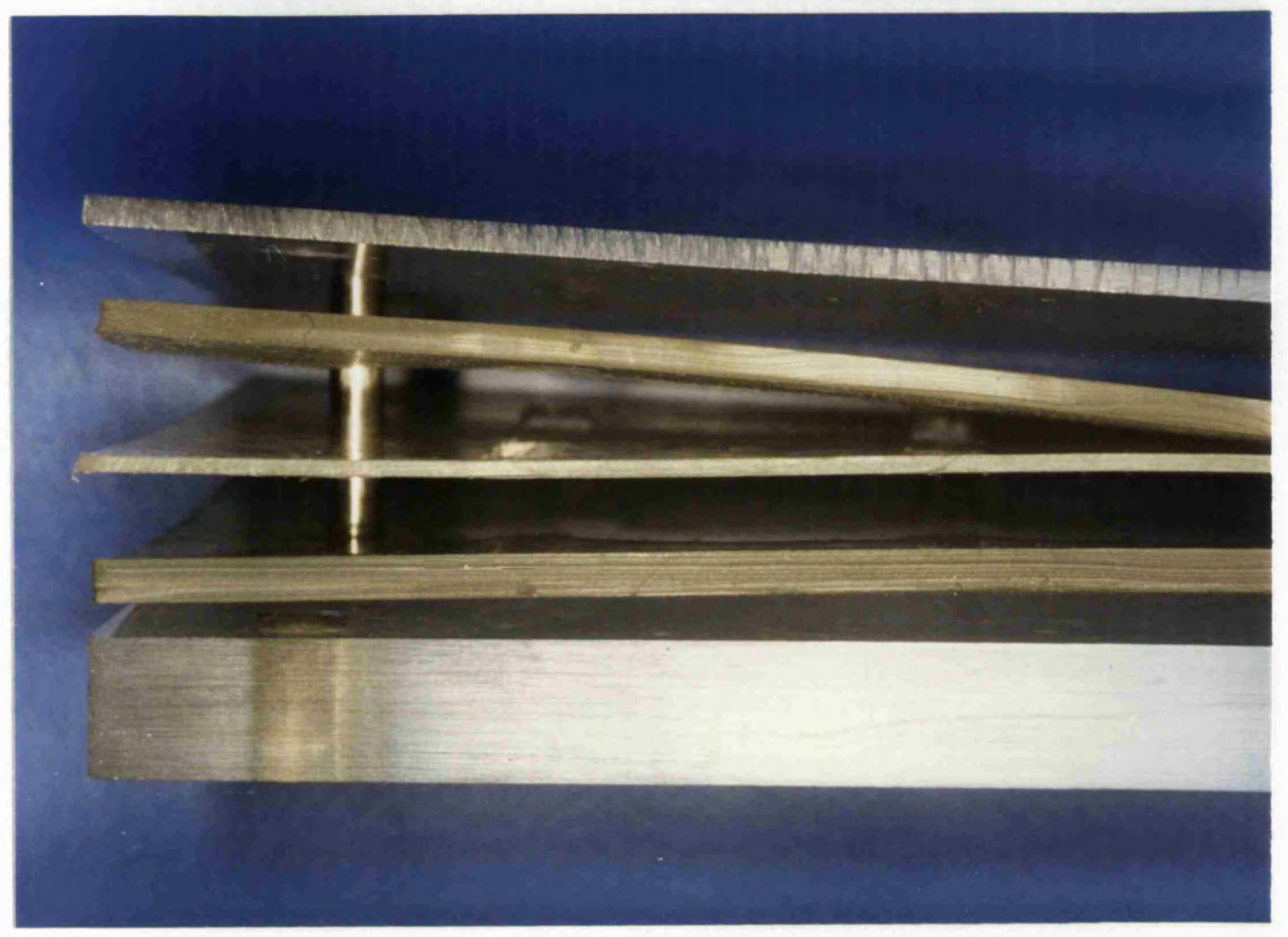

Figure 4.8a : A cross-sectional view of the specimen mould used to fabricate the bonded resin to metal specimens

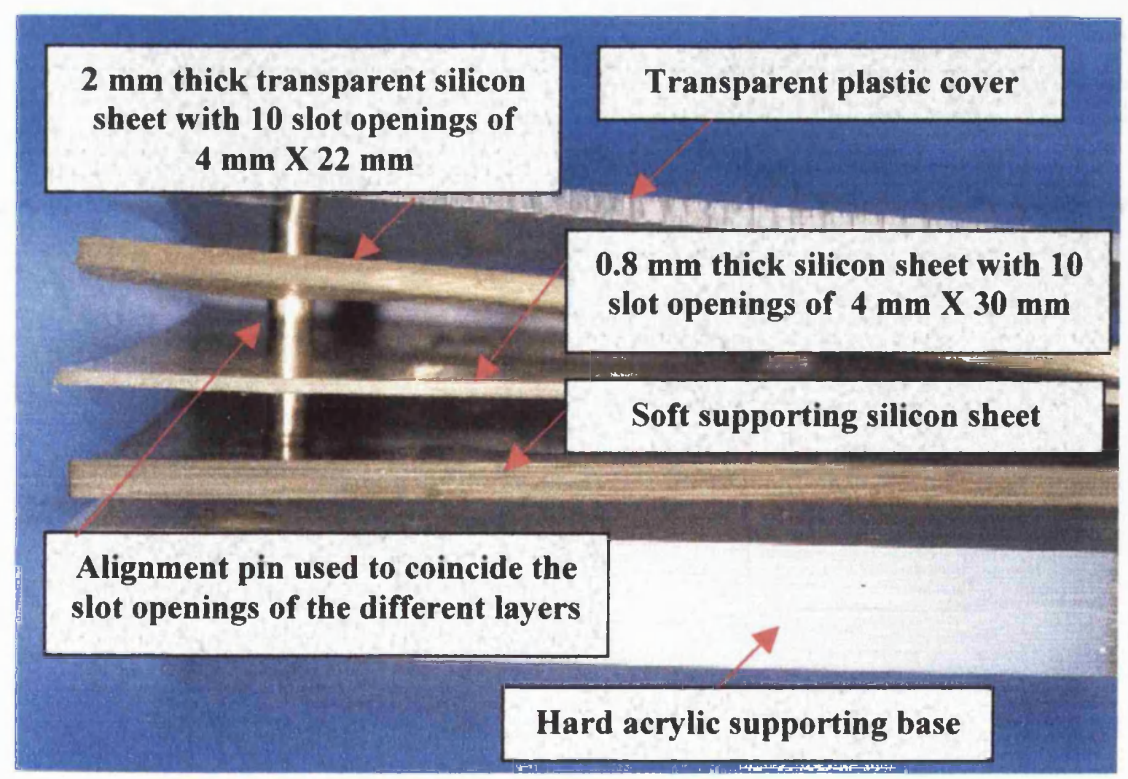

Figure 4.8b : A cross-sectional representation of the different layers of the specimen mould used to fabricate the bonded resin to metal specimens 


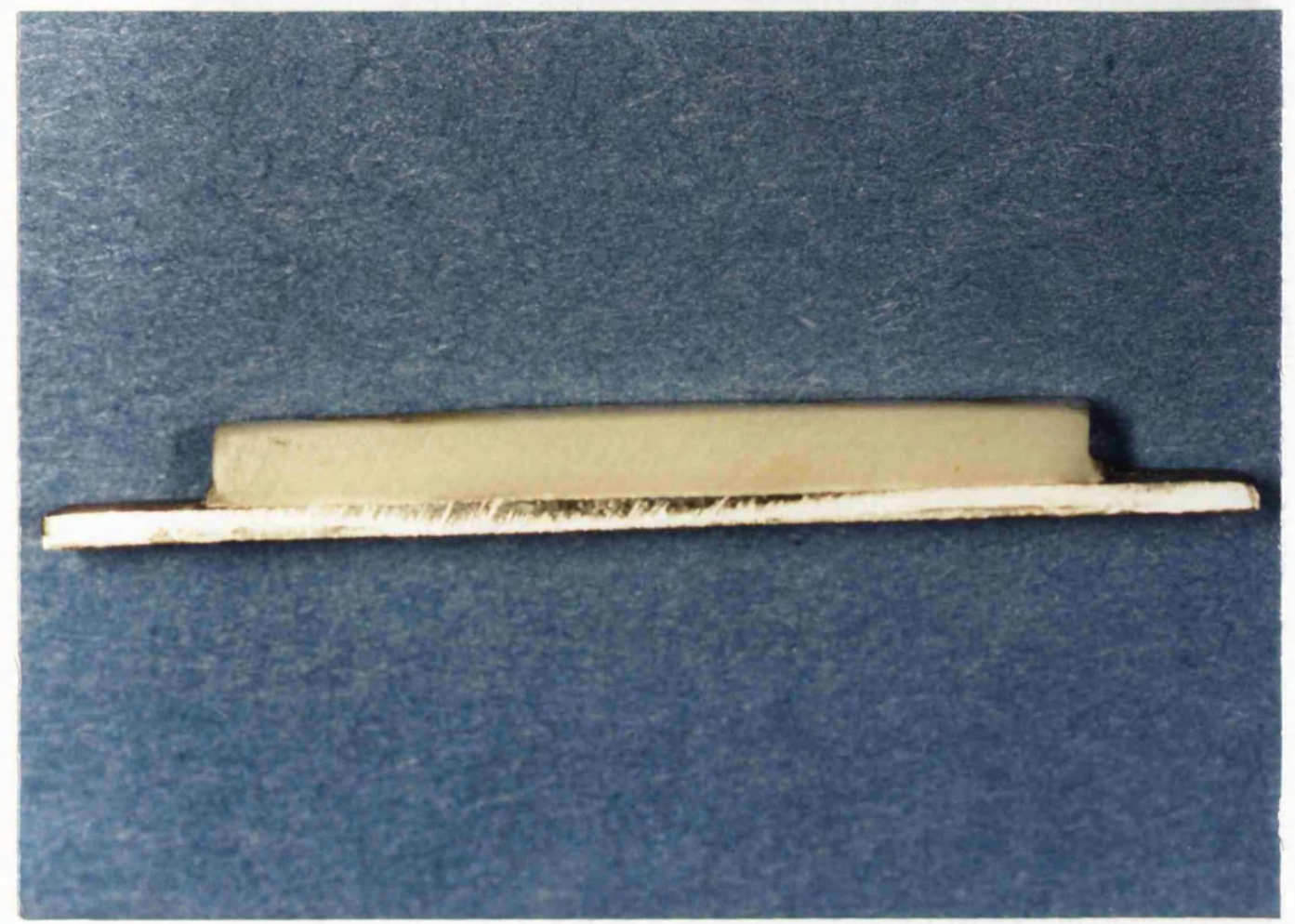

Figure 4.9a : A typical specimen

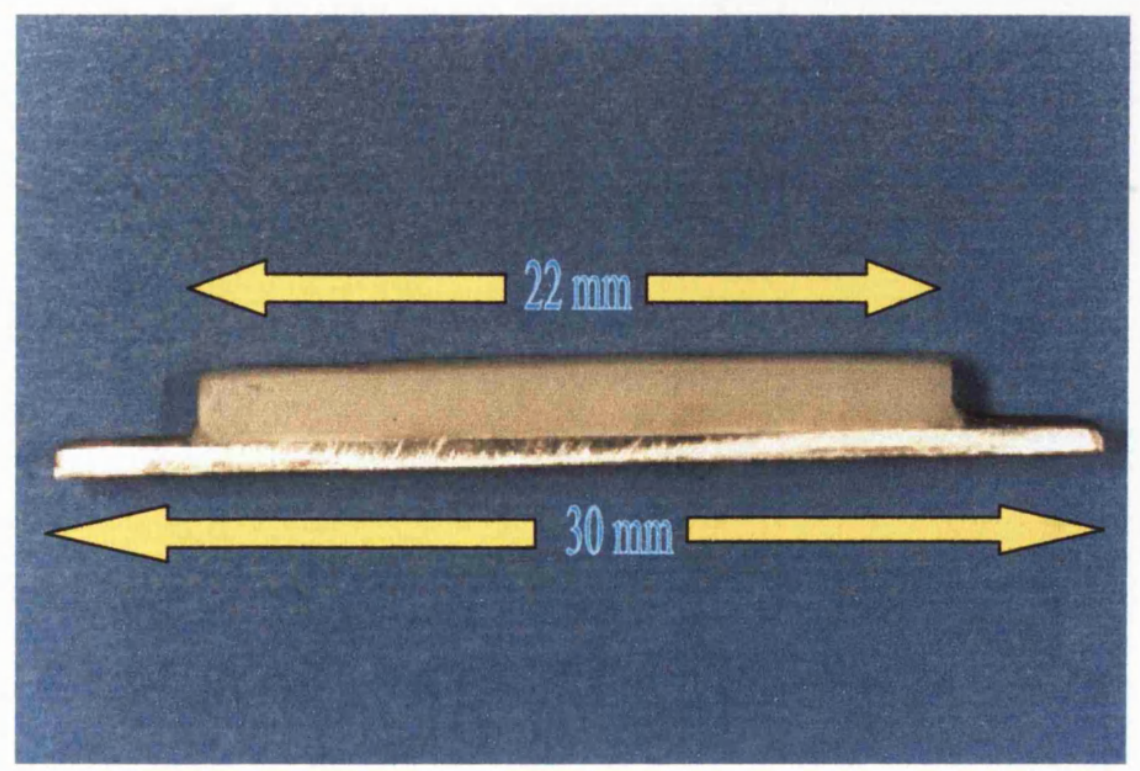

Figure 4.9b : Dimensions of a typical bonded specimen 


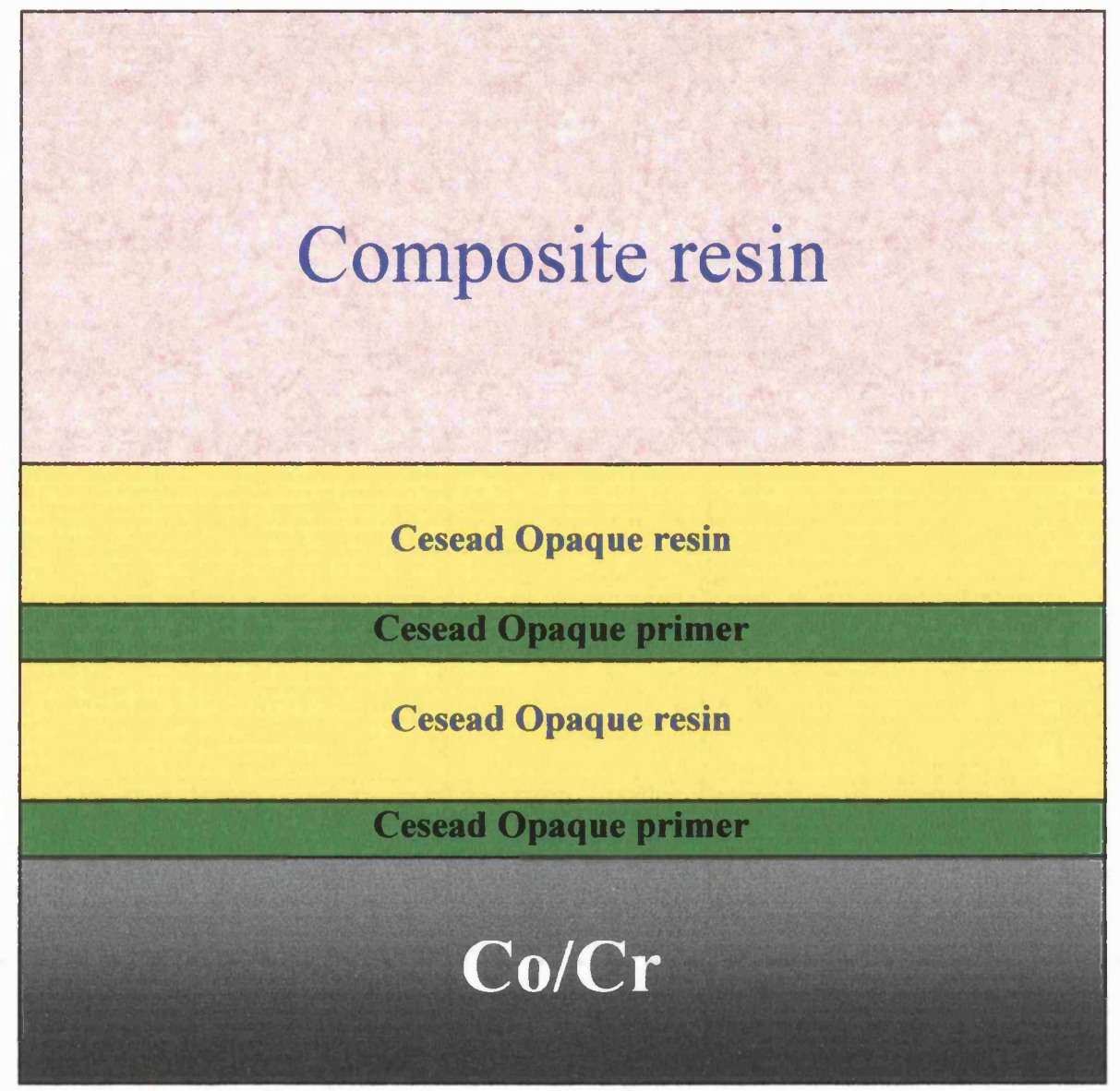

Figure 4.10 : Layers of materials used for the manufacture of specimens 


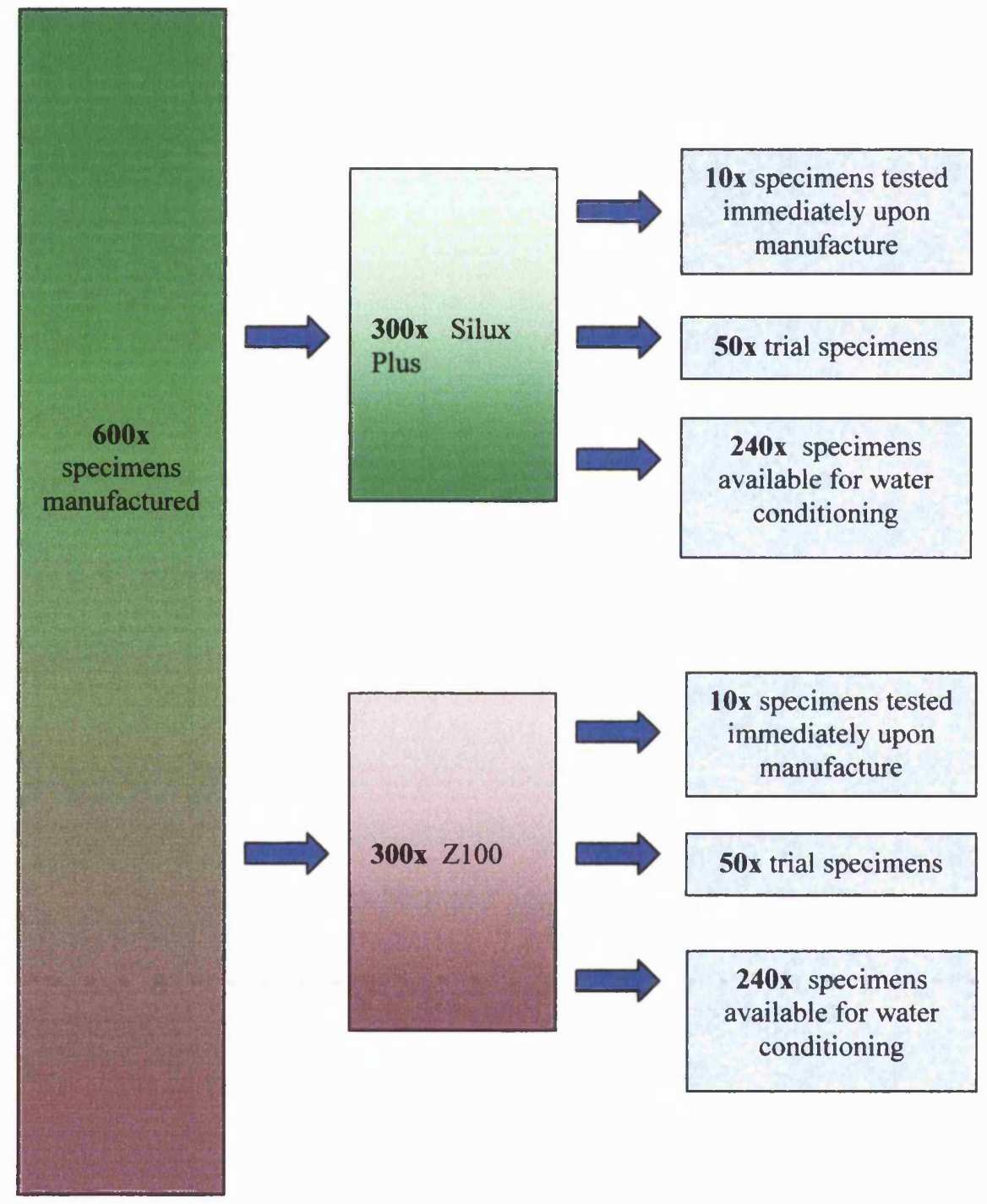

Figure 4.11 : Handling of specimens after manufacture 


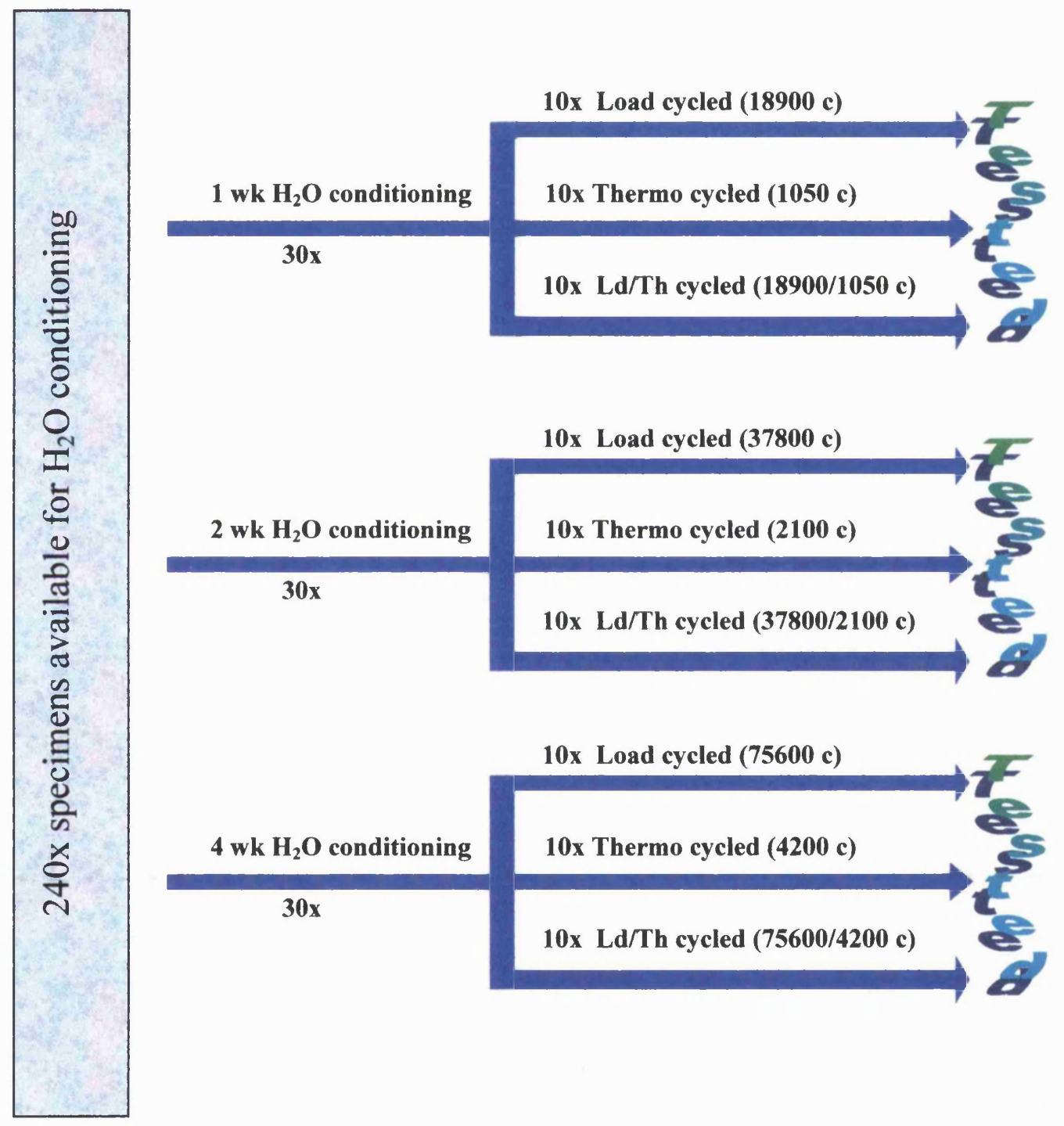

(c=cycles)

(Continued)

Figure 4.12 : Water conditioned specimen bandling 

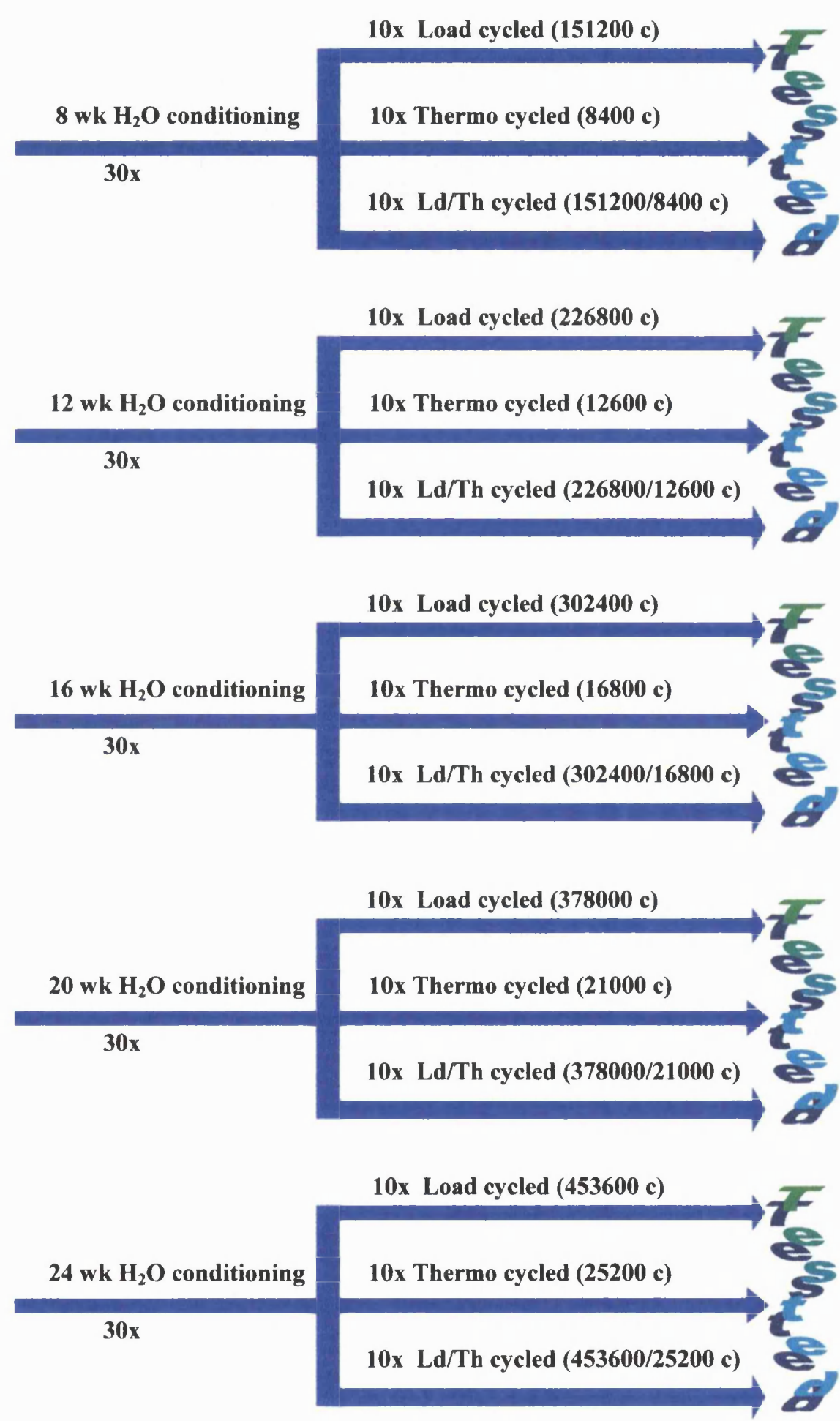


\subsection{THREE POINT BEND TEST OF THE BONDED SPECIMENS (IMMEDIATE GROUP)}

10 specimens of each group were tested to resin fracture in a three point bend test immediately after manufacture in the same universal load testing machine used before in the preliminary study. The crosshead speed was again $2 \mathrm{~mm} / \mathrm{min}$.

A jig was manufactured and set for a span of $25 \mathrm{~mm}$, able to accommodate one specimen at a time by supporting it on either end while the load was applied to the casting surface in the middle of the span (Figure 4.13a). The dimensions of the mounted specimen are indicated in Figure 4.13b. The jig alone is illustrated in Figure 4.14a, and its dimensions indicated in Figure 4.14b.

The width and thickness of the metal component of the laminate specimen were entered into the computer, the jig was placed into the testing machine and the crosshead moved until the load display indicated that minimum force of $0.5 \mathrm{~N}$ was applied to the specimen. The displacement of the crosshead was set at zero and a computer controlled three point bend test was performed on each specimen up to the point of resin failure. Failure was defined from the computer program when there was an abrupt reduction of the load recorded during three point bending. The modulus was calculated from the computer software between a programmed minimum load of $0.1 \mathrm{KN}$ and a maximum of $1 \mathrm{KN}$. Because the dimensions of the metal component only were entered into the software, the value obtained reflected an apparent modulus for the laminates tested since the resins themselves were not loaded directly, but just modified the properties of the metal component. At the point of failure, either a crack within the resin had reached the metal surface registering a cohesive failure, or the resin had debonded from the metal surface 
registering an adhesive failure. Not only was the crack visible, but the fracture or debonding was also heard.

The apparent flexural modulus and stress at failure together with a graphical display of the stress/strain curve were obtained for each specimen as had been obtained for the resin spine specimens. In addition, to the apparent modulus and stress, the extension at failure was also noted.

\subsubsection{DETERMINATION OF THE TESTING CONDITIONS}

\subsection{LOAD CYCLING}

\subsection{DETERMINATION OF THE NUMBER OF LOAD CYCLES}

Assuming 3 periods of 15 minutes of chewing per day, at a chewing rate of 60 cycles per minute $(1 \mathrm{~Hz})$, the average number of chews would be 2,700 times per day. This amounts to roughly 982,800 times per year. It can be argued that not every chewing cycle applies a maximum stress cycle to the structure, nevertheless even a minimum stress cycle will contribute to the fatigue of the prosthesis. 


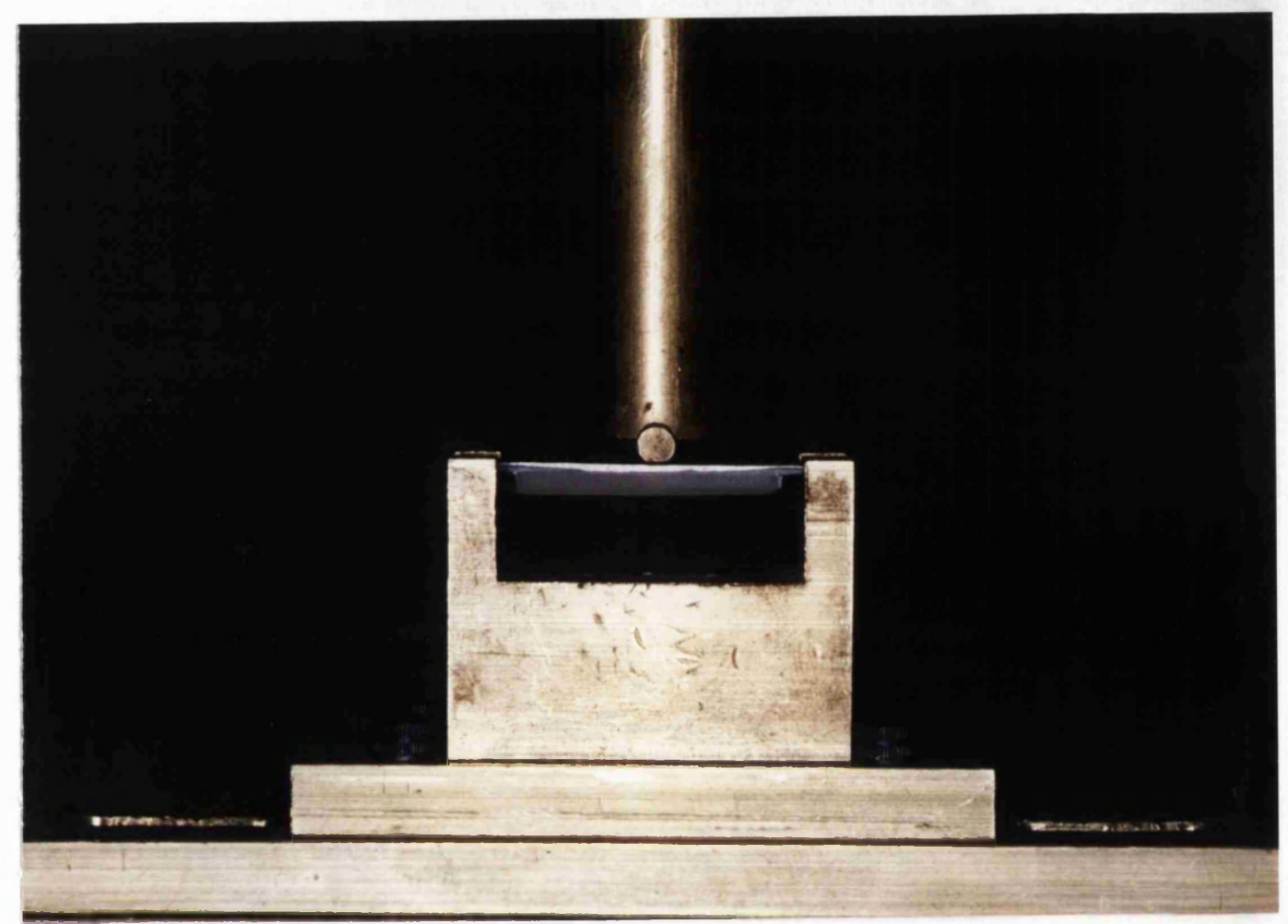

Figure 4.13a : A mounted specimen on the testing jig

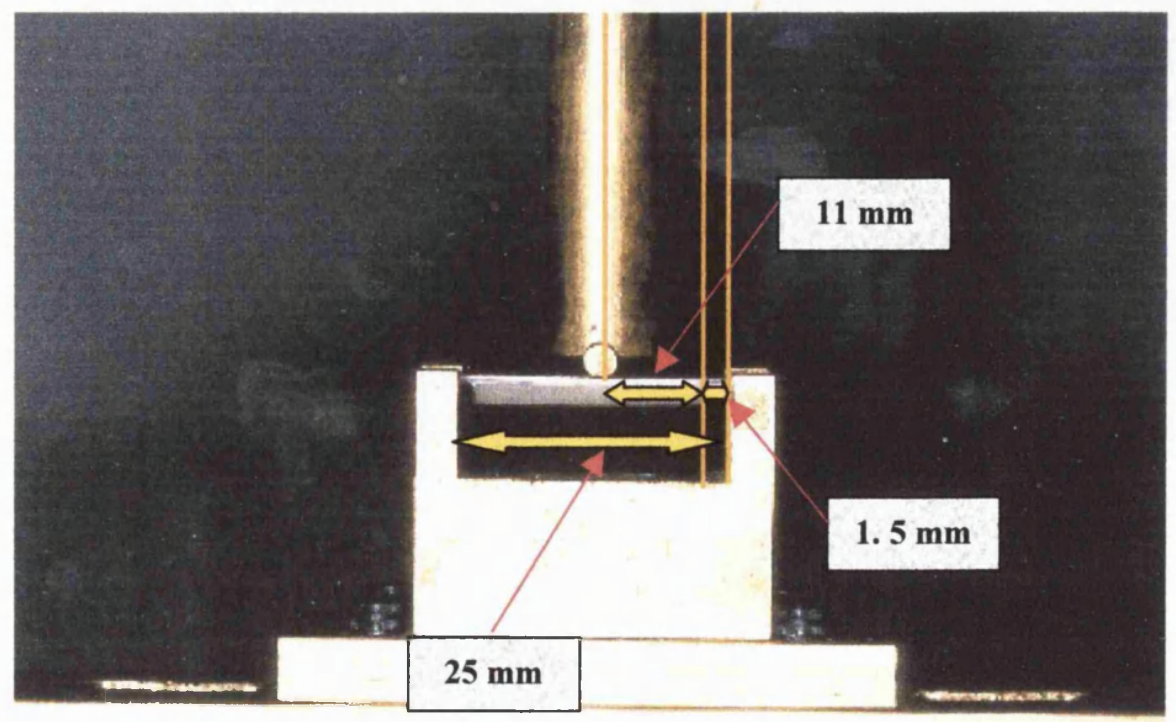

Figure 4.13b : Area of the specimen where load is applied 


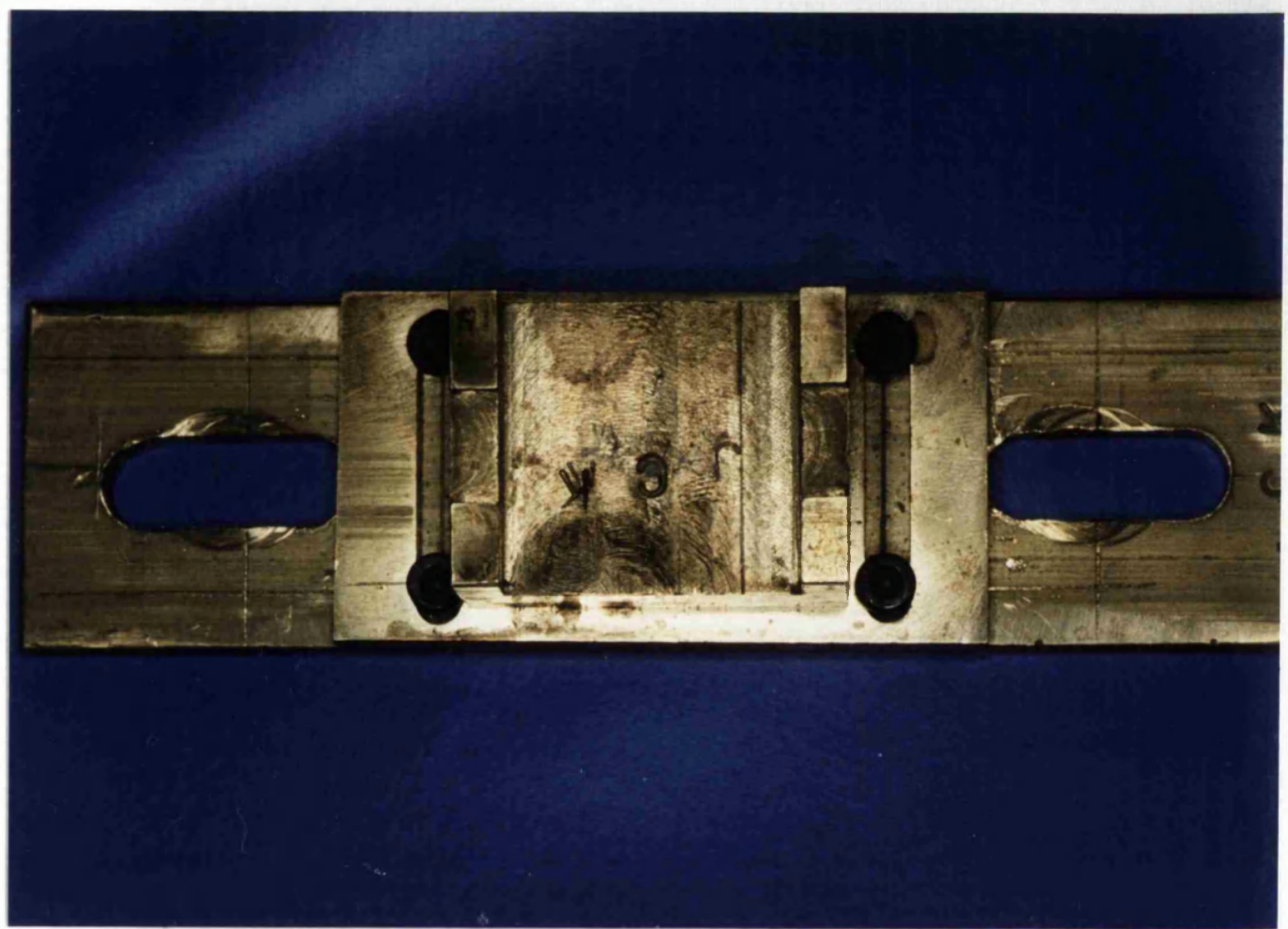

Figure 4.14a : The testing jig

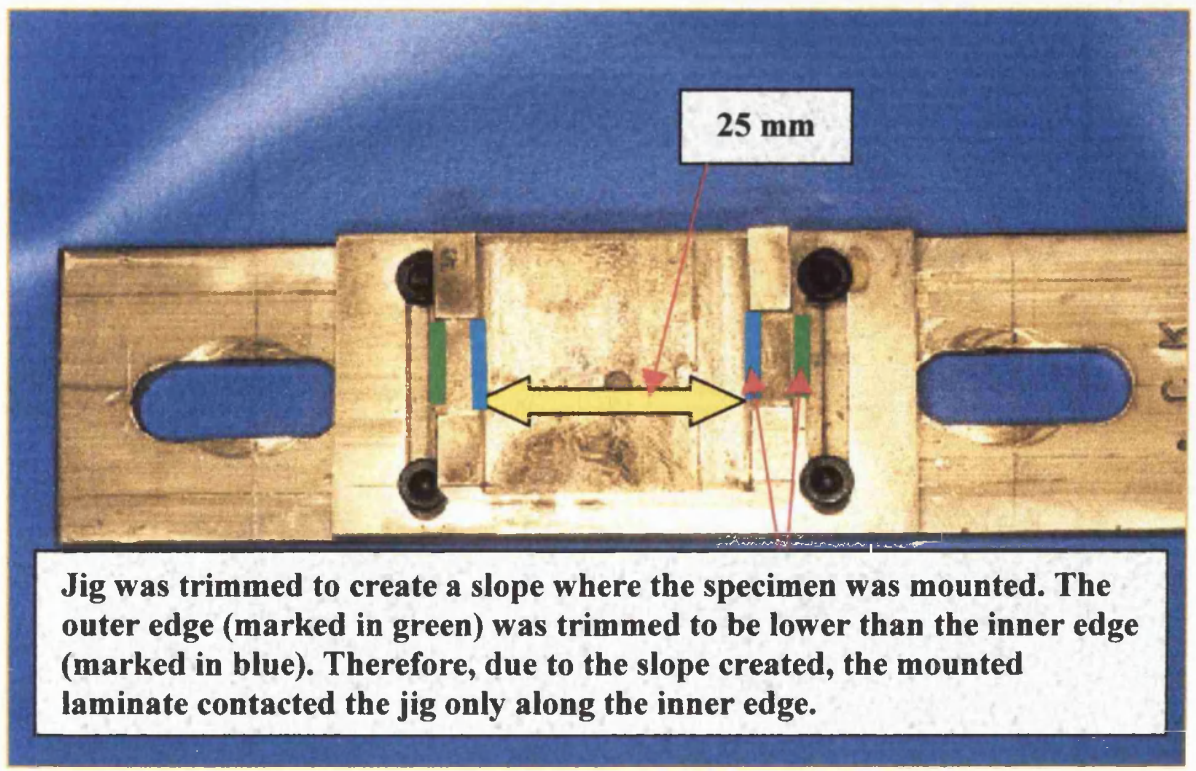

Figure 4.14b : Testing jig dimensions 


\subsection{DETERMINATION OF THE AMOUNT OF LOAD AND CYCLING}

\section{RATE}

Both types of the resin spine/metal specimens were tested in a load cycling regime. 50 Silux Plus/metal and $50 \mathrm{Z100/metal} \mathrm{specimens} \mathrm{forming} \mathrm{the} \mathrm{trial} \mathrm{groups} \mathrm{were} \mathrm{initially}$ evaluated to determine the appropriate load and cycle rate which could be used to test the remaining specimens (Figure 4.11). Both rate of cycling and load levels were determined, using as a reference the load at failure obtained from the three point bend test carried out on the specimens tested immediately after manufacture.

The objective was to determine the maximum possible load level and cycling frequency where fracture of three specimens of the trial group did not occur during 453600 cycles (equal to 24 weeks equivalent intraoral use (Figure 4.12)). Initially, 50\% of the load at failure of the immediate group and a cycling rate of $8 \mathrm{~Hz}$ were used, however all three specimens of each of the materials fractured, while cycling.

The cycle load level was reduced in $5 \%$ increments keeping the frequency stable, down to $20 \%$ of the load value, until fracture of the specimens while cycling was not apparent. If a specimen at any given rate fractured prematurely, then the rate was reduced, $1 \mathrm{~Hz}$ at a time and the same procedure was repeated until three consecutive specimens of the trial group could survive 453600 cycles without fracturing.

A level of $25 \%$ of the load at failure was chosen for both materials after three specimens of each of the materials were cycled for 453600 cycles without an evident fracture at 5 $\mathrm{Hz}$. The water conditioned specimens were divided to 8 groups, each group comprising of 30x Silux Plus specimens and 30x Z100 specimens. Each group was stored in distilled 
water at $37^{\circ} \mathrm{C}$ for a range of $1,2,4,8,12,16,20$ and 24 weeks prior to being subjected to either load cycling, thermo-cycling or load and thermo-cycling (Figure 4.12).

\subsection{THERMO-CYCLING}

\subsection{DETERMINATION OF THE THERMO-CYCLING CONDITIONS}

In order to estimate the amount of time that the specimens would be immersed in the thermo-cycling baths, and the number of cycles, a survey was carried out of the daily eating and drinking habits of 10 adults, during a one week period. From their eating and drinking habits, it was possible to estimate the number of cyclical changes in temperature which occurred during that weekly period. Every minute during a meal an average of 10 boluses of food or sips of drinks were consumed allowing for 6 seconds intraoral presence per bolus or sip. The average duration of a meal was about 20 minutes. The reported estimations of the time spent eating or drinking hot foods and drinks during a meal were 4-5 minutes on average, and that of the time spent eating or drinking cold foods and drinks a further 4-5 minutes. The rest of the time was spent either eating warm food or socializing. 3 meals were consumed during a typical day. It can be calculated, that the consumption of an average of 50 hot and 50 cold boluses or sips form a meal. This indicates that 1050 hot cycles and 1050 cold cycles are equivalent to one week intraorally, $4^{\circ} \mathrm{C}$ and $60^{\circ} \mathrm{C}$ were chosen as the temperatures of the cold and hot baths respectively since they represent typical extreme temperatures of cold and hot foods and drinks consumed routinely. 


\subsubsection{SPECIMEN CONDITIONING}

After the required storage time each group of specimens was subject to: thermo-cycling and/or load cycling.

\subsection{THERMO-CYCLING}

The thermocycler had three baths and a motorised carrier which transported a small specimen holder to the various baths in turn (Figure 4.15a). The temperature of the water was maintained using thermostatic controls (Figure $4.15 \mathrm{~b}$ ). The $37^{\circ} \mathrm{C}$ and $60^{\circ} \mathrm{C}$ baths had heating elements that were thermostatically controlled. To achieve a temperature in the range of $4^{\circ} \mathrm{C}$, this bath was insulated and a cooling element was placed inside the bath. A fine stream of air bubbles within the water was maintained to prevent it from freezing. Distilled water reservoirs were mounted on top of the baths to keep the water levels constant throughout testing. Temperature fluctuation was maintained within $\pm 1^{\circ} \mathrm{C}$ during cycling. Each specimen was thermo-cycled by sequential immersion in distilled water at $4^{\circ} \mathrm{C}$ for 6 seconds, $37^{\circ} \mathrm{C}$ for 6 seconds and $60^{\circ} \mathrm{C}$ for 6 seconds and $37^{\circ} \mathrm{C}$ for 6 seconds. Total cycle time was 85 seconds. This took account of the time needed for the specimen carrier to travel between the water baths. For the purpose of this study, each specimen of the thermo-cycled groups undertook 1050 cycles for each week of water storage time (Figure 4.12). 


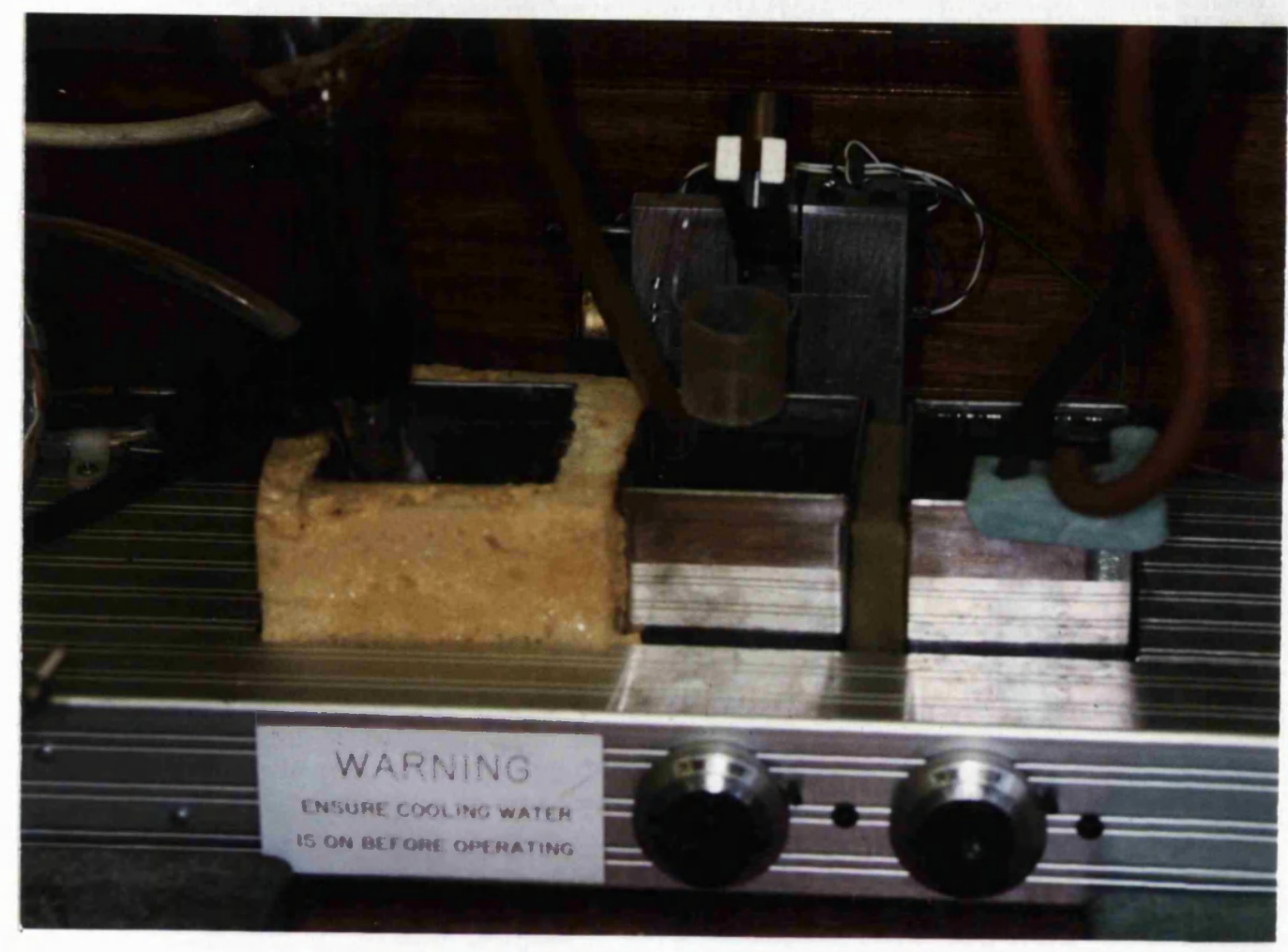

Figure 4.15a : The thermo-cycling machine

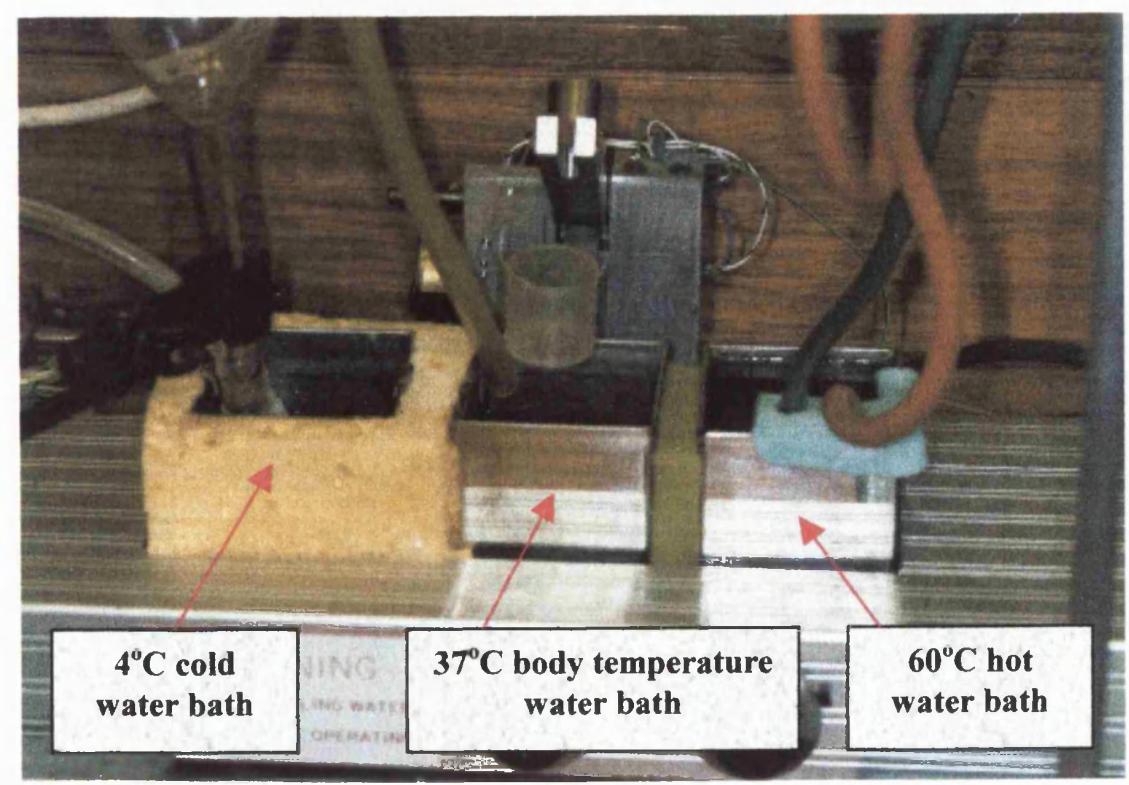

Figure 4.15b : The different water baths of the thermo-cycling machine 


\subsection{LOAD CYCLING}

Each specimen was load cycled for 18900 cycles for each week of water storage to which it was exposed (Figure 4.12).

The cyclical loading apparatus consisted of four components.

a) An oscillator (Bruel \& Kjoer $4812^{*}$ ) which included a permanent magnet together with an electromagnetic coil inside it.

b) A signal generator (Thandar TG502**) which fed an appropriate current into the electromagnetic coil, causing it to oscillate.

c) A static load cell(RDP $\left.41 / 8186-03^{* * *}\right)$ used to register the forces exerted onto the specimen.

d) A computer terminal running a customised software application used to monitor the amplitude and shape of the wave form of the load cycle.

The load cycling set-up used was able to maintain constant loading during cycling with a constant amplitude sinusoidal wave form on each cycle, at a frequency of $5 \mathrm{~Hz}$. During cyclical loading the specimens were mounted on the jig shown in Figures 4.13a and $4.13 \mathrm{~b}$, as described previously.

"Bruel \& Kjoer, Copenhagen, Denmark

** Thandar Electronics Ltd, Huntington, England

*** RDP Electronics,London, England. 


\subsubsection{THREE POINT BEND TEST OF THE CONDITIONED SPECIMENS}

Once load and thermo-cycling were completed, the specimens were tested to failure using the universal load testing machine (Instron) operated at a crosshead speed of $2 \mathrm{~mm} / \mathrm{min}$. All specimens were subjected to a three point bend test as described previously.

\subsubsection{STATISTICAL ANALYSIS}

The results obtained were analysed using descriptive statistical calculations. Mean, median and standard deviation values have been calculated for each material at every time group. In addition, analysis of the data for each group for each material in between the various time intervals and also analysis of the data of each group in relation to each other for both materials within the same week period using the Mann - Whitney test were also performed. Non parametric tests were used for the main statistical evaluation since the sample size for each experimental group was small and the results were potentially not normally distributed.

Further, in order to verify differences between groups associated with the different test durations, Mann-Whitney tests were performed between the total population of specimens (i.e. Load cycled + Thermo-cycled + Load/thermo-cycled) of each week. To investigate possible changes in the behaviour of one particular test group in relation to other specimens of the same week a similar statistical evaluation was done comparing the individual group eg. Load cycled, to the total specimen population of the same week.

\subsubsection{DYE PENETRATION STUDY}

The fractured specimens from the $1,2,16,20$ and 24 week groups were immersed in methylene blue dye to investigate dye penetration. They were stored in the solution for 24 hours. After this they were washed and dried prior to examination under an optical 
microscope in order to note the fracture lines and determine the mode of failure. Specimens were placed on the specimen stage of the light microscope (Wild M400*) and their position noted with the aid of the vernier scale on the stage. The extent of dye penetration was assessed at $32 \mathrm{x}$ magnification with the aid of the cross-wire of the eyepiece and the vernier controls on the stage.

In addition, a further 2 specimens from each of these test groups were also prepared for examination using a Scanning Electron Microscope. The specimens were evaluated after all dye was washed off. They were then desiccated and then sputter coated with gold palladium under vacuum (S150A Sputter Coater ${ }^{* *}$ ). They were examined in an SEM (Cambridge $90 \mathrm{~B}^{* * *}$ ) at an accelerating voltage of $15 \mathrm{kV}$.

\footnotetext{
"Wild Heerbrugg Ltd, CH-9435, Heerbrugg, Switzerland

** Edwards Instruments, Eastbourne, England

*** Cambridge Instruments Ltd, Clifton Road, Cambridge, CB1 3QH
} 


\section{RESULTS}

\subsection{PRELIMINARY STUDY}

\subsubsection{FLEXURAL TESTING TO DETERMINE THE MECHANICAL} PROPERTIES OF THE COMPONENTS

Prior to testing the combined metal/composite specimens, $10 \mathrm{Co} / \mathrm{Cr}$ metal components as prepared for lamination, 10 Silux Plus and $10 \mathrm{Z100}$ composite resin spines of the laminate specimens were tested individually in order to obtain the relevant mechanical properties of each component of the experimental system. These manufactured metal and resin components, had the same dimensions as of the components of the bonded laminate specimens. The results are shown in Tables 5.1, 5.2 and 5.3.

\begin{tabular}{|l|c|}
\hline & $\begin{array}{c}\text { Modulus } \\
\text { (GPa) }\end{array}$ \\
\hline Mean & 30.4 \\
S/d* & 3.2 \\
Median & 31.1 \\
\hline
\end{tabular}

Table $5.1: \mathrm{Co} / \mathrm{Cr}$ alloy as prepared for lamination (flexural testing)

\begin{tabular}{|l|c|c|}
\hline & $\begin{array}{c}\text { Modulus } \\
\text { (GPa) }\end{array}$ & $\begin{array}{c}\text { Stress at failure } \\
\text { (MPa) }\end{array}$ \\
\hline Mean & 2.6 & 41.5 \\
S/d & 0.3 & 9.5 \\
Median & 2.7 & 37.6 \\
\hline
\end{tabular}

Table 5.2 : Silux Plus resin spines (flexural testing)

$\mathrm{S} / \mathrm{d}=$ Standard deviation 


\begin{tabular}{|l|c|c|}
\hline & $\begin{array}{c}\text { Modulus } \\
\text { (GPa) }\end{array}$ & $\begin{array}{c}\text { Stress at failure } \\
\text { (MPa) }\end{array}$ \\
\hline Mean & 8.7 & 104.0 \\
S/d & 1.9 & 28.3 \\
Median & 8.9 & 109.7 \\
\hline
\end{tabular}

Table $5.3: \mathrm{Z100}$ resin spines (flexural testing)

\subsubsection{VARIATION IN THE MASS WEIGHING PROCEDURE}

A metal surgical razor blade was weighed at ten different times on three different days and the variation in the values recorded was calculated. During the interim period the razor was stored in a desiccator. The values recorded are shown in Table 5.4. The variation in the values indicated the effects of setting up the balance before each weighing and the environmental variations around the balance. It was determined that any mass changes in excess of 0.0009 grams were outside the limits of experimental variation. 


\begin{tabular}{|l|c|c|c|}
\hline & \multicolumn{3}{|c|}{ Mass in grams } \\
\hline & Day 1 & Day 2 & Day 3 \\
\cline { 2 - 4 } & 1.6217 & 1.6214 & 1.6230 \\
& 1.6207 & 1.6226 & 1.6208 \\
& 1.6218 & 1.6227 & 1.6228 \\
& 1.6210 & 1.6215 & 1.6210 \\
& 1.6209 & 1.6228 & 1.6226 \\
& 1.6223 & 1.6209 & 1.6209 \\
& 1.6202 & 1.6230 & 1.6229 \\
& 1.6228 & 1.6214 & 1.6203 \\
& 1.6205 & 1.6223 & 1.6230 \\
\cline { 1 - 3 } Mean & 1.6227 & 1.6222 & 1.6211 \\
\hline S/d & 1.6215 & 1.6221 & 1.6218 \\
\cline { 1 - 2 } Mean value of mean & 1.6218 & & 0.0011 \\
\hline Mean value of S/d & $\mathbf{0 . 0 0 0 9}$ & & \\
\hline
\end{tabular}

Table 5.4 : Mass of metal blade to establish variation in weighing procedure

\subsubsection{WATER SORPTION AND SOLUBILITY OF THE RESIN COMPONENT}

The water sorption and solubility study required the precise weighing of specimens after their immersion in water for specific time periods. The specimens underwent two sorption/desorption cycles. During the first cycle a combination of sorption and solubility would have occurred whereas it was assumed on the second cycle, sorption alone occurred as soluble material would have been lost during the first cycle. This may be verified at the end of the second cycle when starting and end mass should be equal, within the experimental variation of the weighing procedure. On both cycles, a scatter distribution of the masses, the percentage water sorption and the increase in the mass against the $t^{1 / 2}$ were evaluated. A value for solubility was also calculated at the end of the 
second cycle by comparing initial mass prior to cycle one and the final desiccated mass at the end of cycle two.

\subsubsection{SILUX PLUS}

A scatter plot of the mass of each Silux Plus specimen during the first cycle is shown in Figure 5.1.

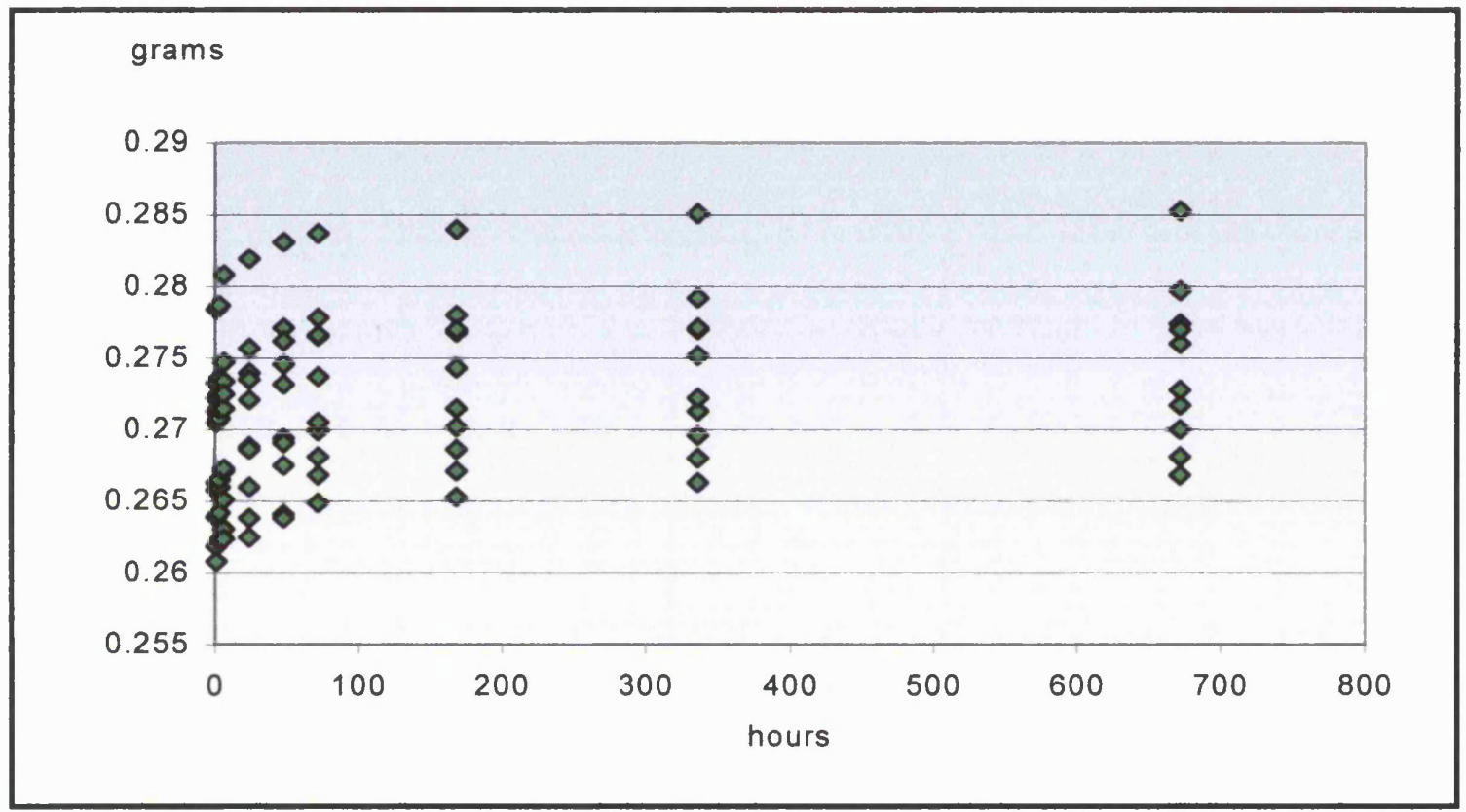

Figure 5.1 : Silux Plus: Scatter distribution of individual specimens mass $\left(1^{\text {st }}\right.$ cycle)

The results of the Silux Plus first sorption cycle are set out in Table 5.5 and Figure 5.2.

\begin{tabular}{|l|c|c|c|c|c|c|c|}
\hline Hours & $\mathbf{0}$ & $\mathbf{1}$ & $\mathbf{3}$ & $\mathbf{6}$ & $\mathbf{2 4}$ & $\mathbf{4 8}$ & $\mathbf{7 2}$ \\
\hline Mean \% ws & 0.00 & 0.20 & 0.35 & 0.70 & 1.04 & 1.45 & 1.84 \\
S/d & 0.00 & 0.07 & 0.14 & 0.12 & 0.22 & 0.25 & 0.26 \\
\hline Hours & $\mathbf{1 6 8}$ & $\mathbf{3 3 6}$ & $\mathbf{6 7 2}$ & $\mathbf{1 0 0 8}$ & $\mathbf{1 3 4 4}$ & $\mathbf{1 6 8 0}$ & $\mathbf{2 0 1 6}$ \\
\hline Mean \% ws & 2.00 & 2.31 & 2.45 & 2.58 & 2.65 & 2.68 & 2.69 \\
S/d & 0.25 & 0.22 & 0.17 & 0.16 & 0.18 & 0.15 & 0.16 \\
\hline
\end{tabular}

Table 5.5 : Silux Plus water sorption (ws)

$\left(1^{\text {st }}\right.$ cycle: Mean $\%$ values) 
$\%$ water sorption

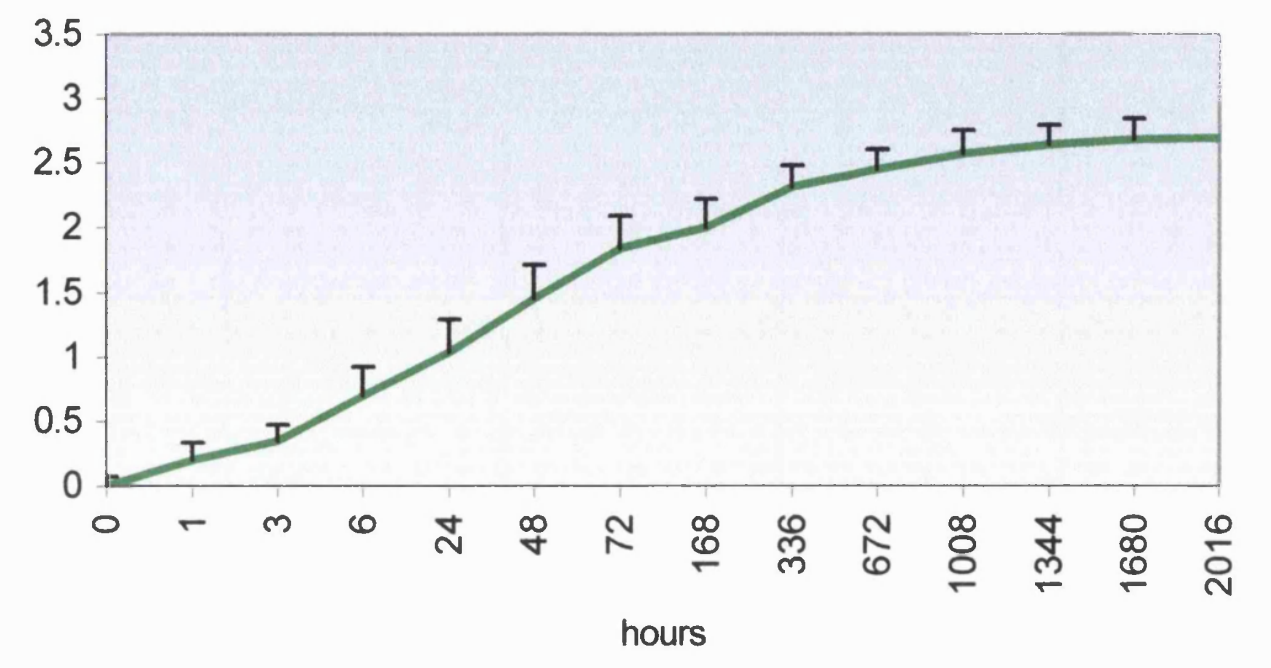

Figure 5.2 : Silux Plus: Mean water sorption $\left(1^{\text {st }}\right.$ cycle $)$

The water sorption was rapid, and by the end of one week nearly $75 \%$ of the total sorption in the cycle had occurred. Equilibrium was achieved at around 6 weeks (1008 hours). The total mean water uptake was $2.69 \%$. The increase in the mass of Silux Plus during the first cycle was plotted against $\mathrm{t}^{1 / 2}$ (Figure 5.3).

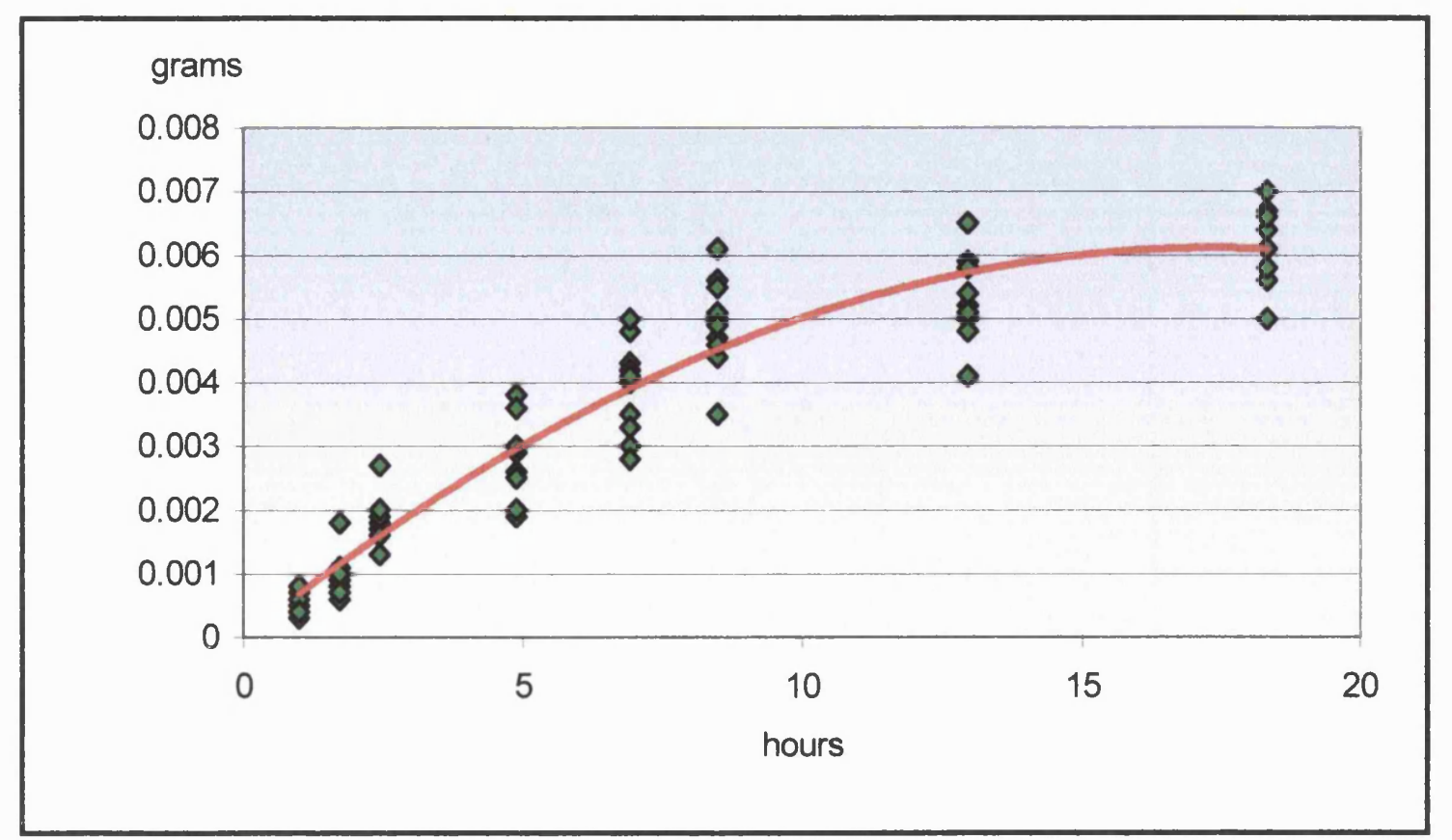

Figure 5.3 : Silux Plus: Increase in mass with respect to $t^{1 / 2}\left(1^{\text {st }}\right.$ cycle $)$ 
A scatter plot of the mass of each Silux Plus specimen during the second cycle is shown in Figure 5.4.

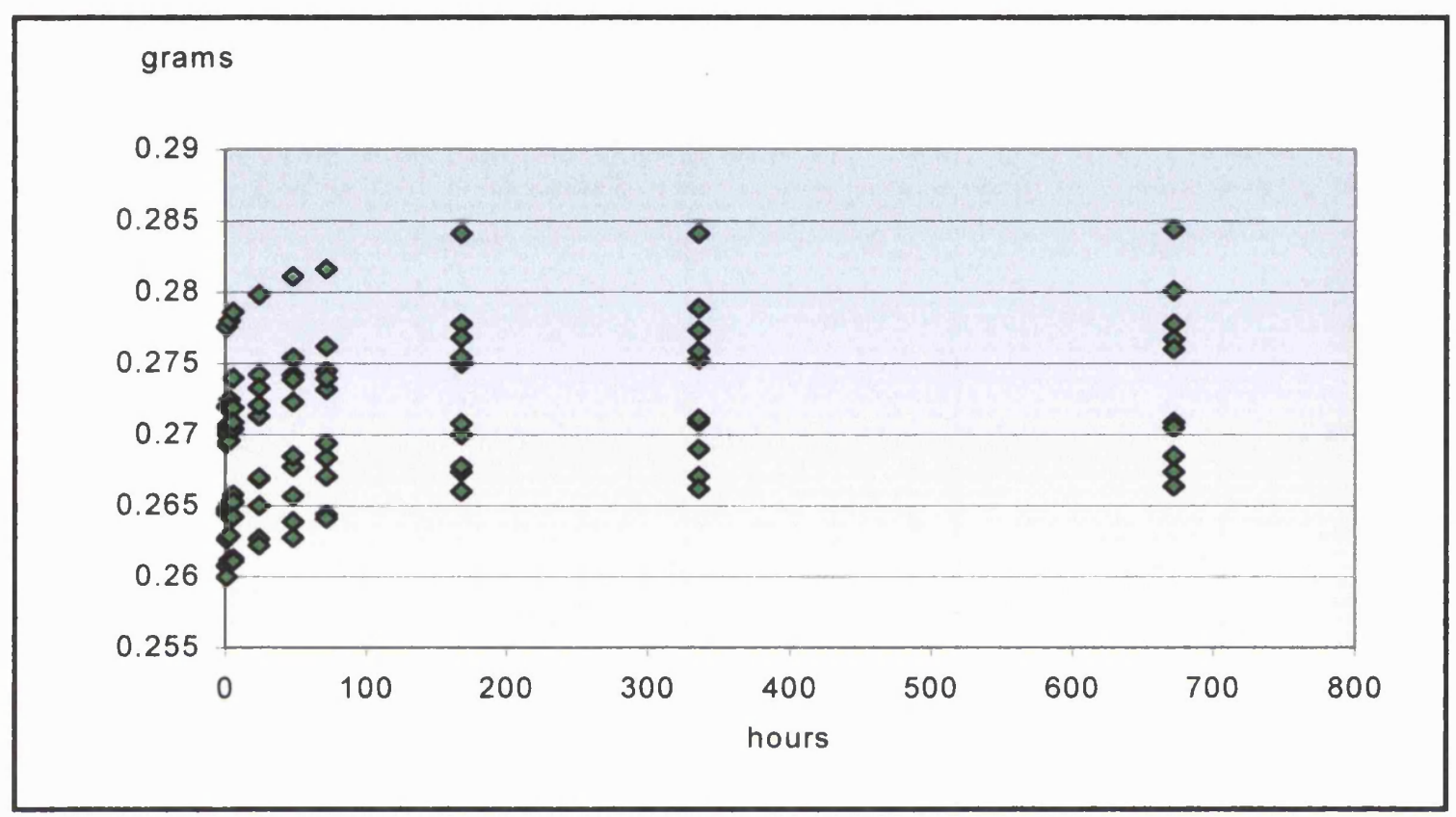

Figure 5.4 : Silux Plus: Scatter distribution of individual specimens mass $\left(2^{\text {nd }}\right.$ cycle)

Silux Plus during the second cycle showed a water uptake performance which is displayed in Table 5.6 and Figure 5.5.

\begin{tabular}{|l|c|c|c|c|c|c|c|}
\hline Hours & $\mathbf{0}$ & $\mathbf{1}$ & $\mathbf{3}$ & $\mathbf{6}$ & $\mathbf{2 4}$ & $\mathbf{4 8}$ & $\mathbf{7 2}$ \\
\hline Mean \%ws & 0.00 & 0.36 & 0.56 & 0.78 & 1.19 & 1.61 & 1.88 \\
S/d & 0.00 & 0.06 & 0.14 & 0.12 & 0.10 & 0.14 & 0.14 \\
\hline Hours & $\mathbf{1 6 8}$ & $\mathbf{3 3 6}$ & $\mathbf{6 7 2}$ & $\mathbf{1 0 0 8}$ & $\mathbf{1 3 4 4}$ & $\mathbf{1 6 8 0}$ & $\mathbf{2 0 1 6}$ \\
\hline Mean \%ws & 2.57 & 2.74 & 2.85 & 2.80 & 2.80 & 2.87 & 2.88 \\
S/d & 0.22 & 0.11 & 0.22 & 0.20 & 0.19 & 0.18 & 0.19 \\
\hline
\end{tabular}

Table 5.6 : Silux Plus water sorption (ws)

$\left(2^{\text {nd }}\right.$ cycle: Mean $\%$ values) 
$\%$ water sorption

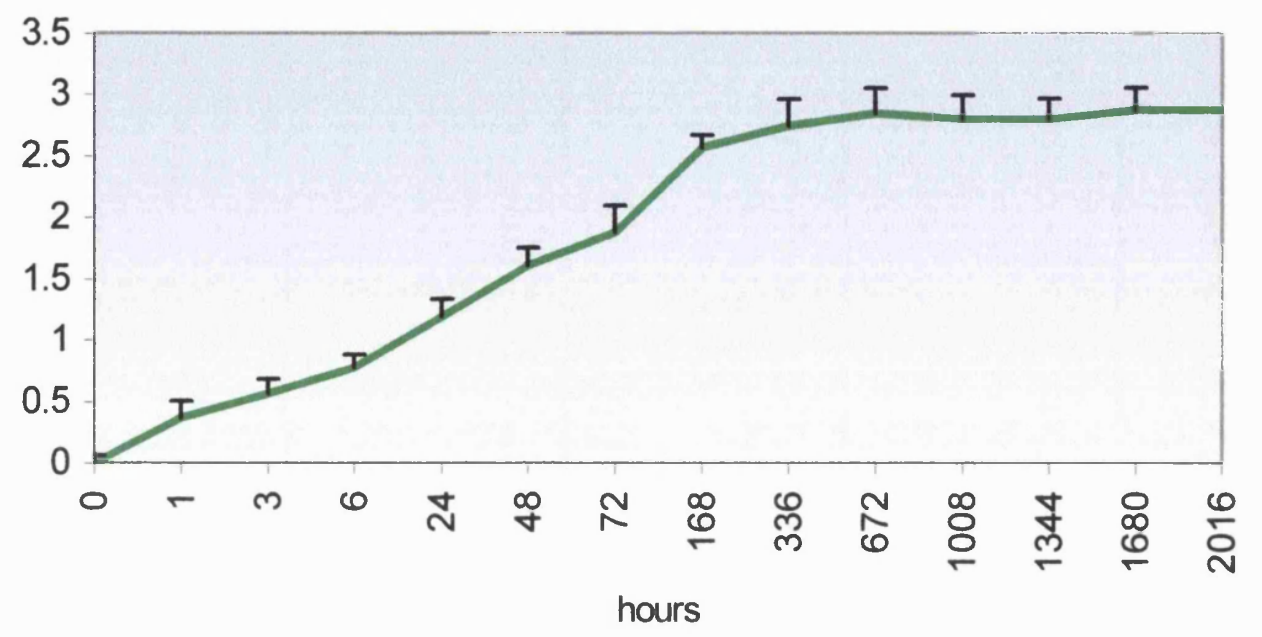

Figure 5.5 : Silux Plus: Mean water sorption $\left(2^{\text {nd }}\right.$ cycle $)$

The pattern observed in the second sorption cycle was slightly different. Water uptake was rapid and equilibrium was achieved, in this case, after four weeks (672 hours). Nearly $90 \%$ of the sorption had occurred by the end of week one (168 hours). The final value for sorption in this case was $2.88 \%$. The increase in the mass of Silux Plus during the second cycle was plotted against $t^{1 / 2}$ (Figure 5.6).

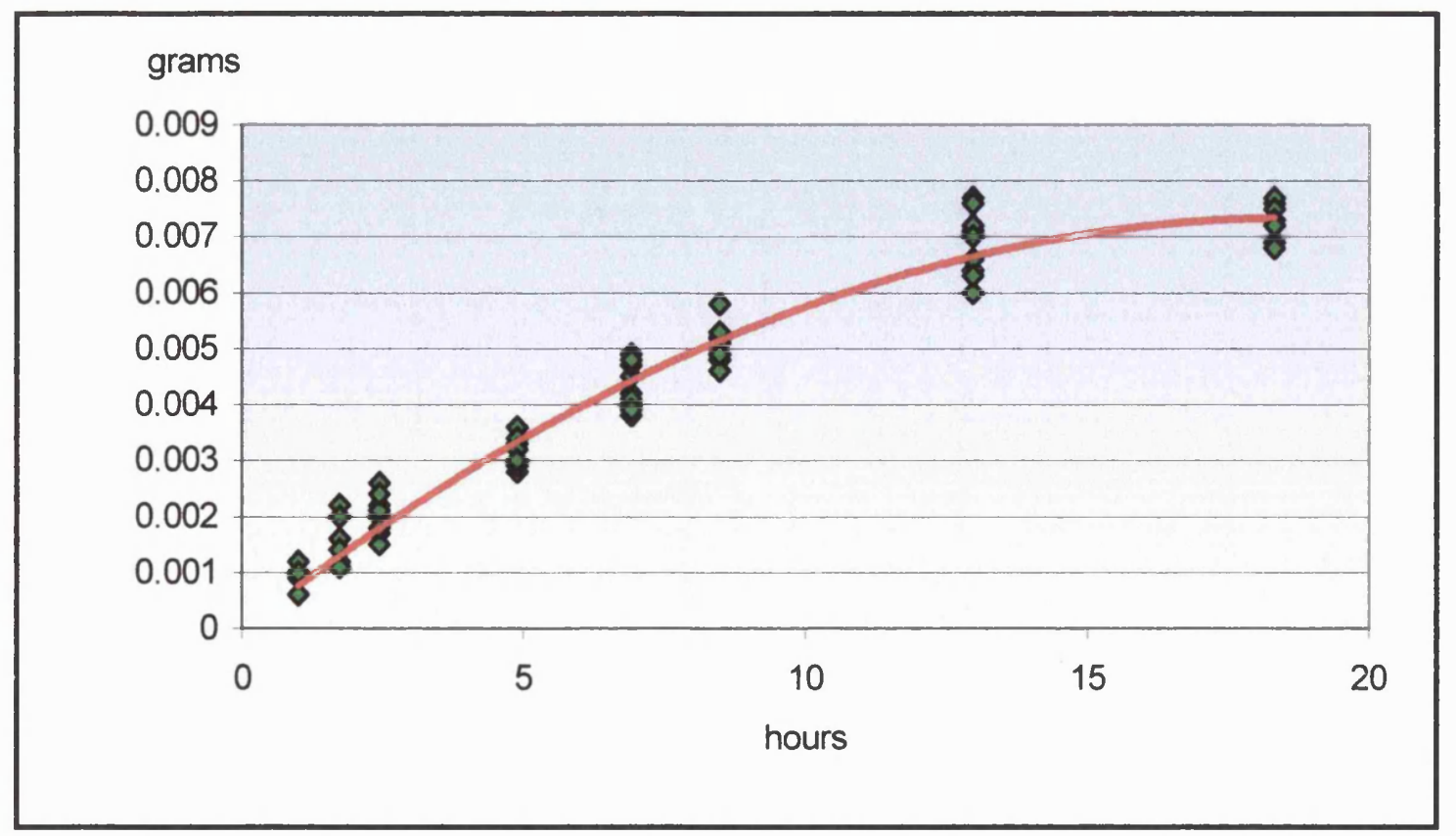

Figure 5.6 : Silux Plus: Increase in mass with respect to $t^{1 / 2}\left(2^{\text {nd }}\right.$ cycle $)$ 
The calculated solubility value and standard deviation for Silux Plus is shown in Table 5.7 .

\begin{tabular}{|l|c|}
\hline & Silux Plus \\
\hline Mean \% solubility & 0.61 \\
\hline S/d (\%) & 0.07 \\
\hline
\end{tabular}

Table 5.7 : Solubility (Silux Plus mean \% values)

There was a mean mass loss of $0.0163 \mathrm{~g}$ between the start and the end of the first cycle. No further loss was observed at the second cycle.

The specimen's mass changes were monitored, and compared to the experimental variation. After the maximum sorption was achieved for the second time, the specimens were again placed in the desiccator and kept until completely desiccated. The specimens were then weighed again and their changes were again compared to the experimental variation. The values obtained were similar (within the experimental variation) to those obtained at the start of the second sorption cycle. 


\subsubsection{Z100}

A scatter plot of the mass of each Z100 specimen during the first cycle is shown in Figure 5.7 .

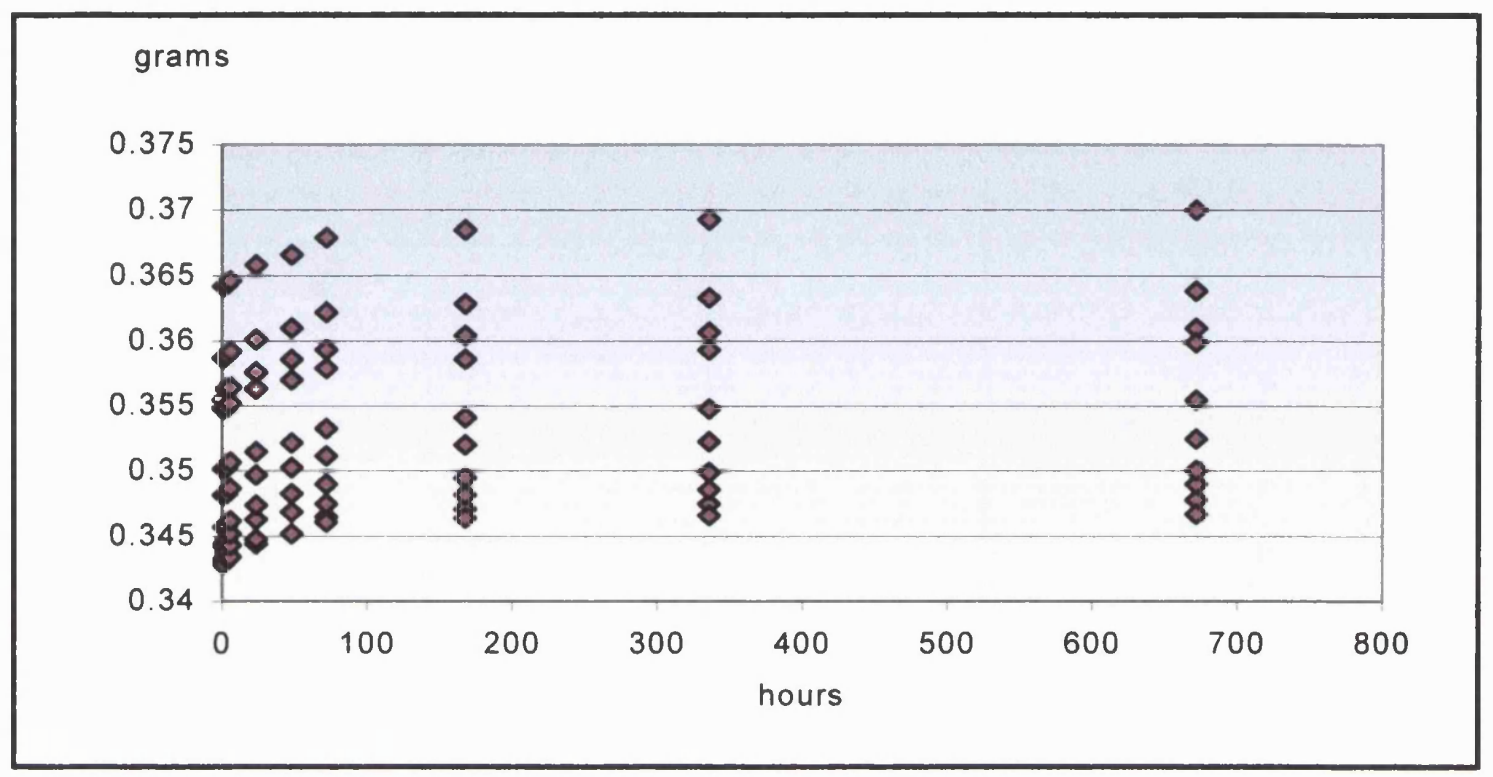

Figure 5.7 : Z100: Scatter distribution of individual specimens mass $\left(1^{\text {st }}\right.$ cycle $)$

The results of the Z100 first water sorption cycle are set out in Table 5.8 and Figure 5.8.

\begin{tabular}{|l|c|c|c|c|c|c|c|}
\hline Hours & $\mathbf{0}$ & $\mathbf{1}$ & $\mathbf{3}$ & $\mathbf{6}$ & $\mathbf{2 4}$ & $\mathbf{4 8}$ & $\mathbf{7 2}$ \\
\hline Mean \% ws & 0.00 & 0.07 & 0.16 & 0.25 & 0.54 & 0.75 & 1.02 \\
S/d & 0.00 & 0.03 & 0.11 & 0.13 & 0.10 & 0.10 & 0.08 \\
\hline Hours & $\mathbf{1 6 8}$ & $\mathbf{3 3 6}$ & $\mathbf{6 7 2}$ & $\mathbf{1 0 0 8}$ & $\mathbf{1 3 4 4}$ & $\mathbf{1 6 8 0}$ & $\mathbf{2 0 1 6}$ \\
\hline Mean \% ws & 1.21 & 1.35 & 1.46 & 1.53 & 1.59 & 1.61 & 1.64 \\
S/d & 0.10 & 0.10 & 0.14 & 0.16 & 0.18 & 0.19 & 0.19 \\
\hline
\end{tabular}

Table 5.8 : Z100 water sorption (ws) $\left(1^{\text {st }}\right.$ cycle: Mean $\%$ values $)$ 


\section{$\%$ water sorption}

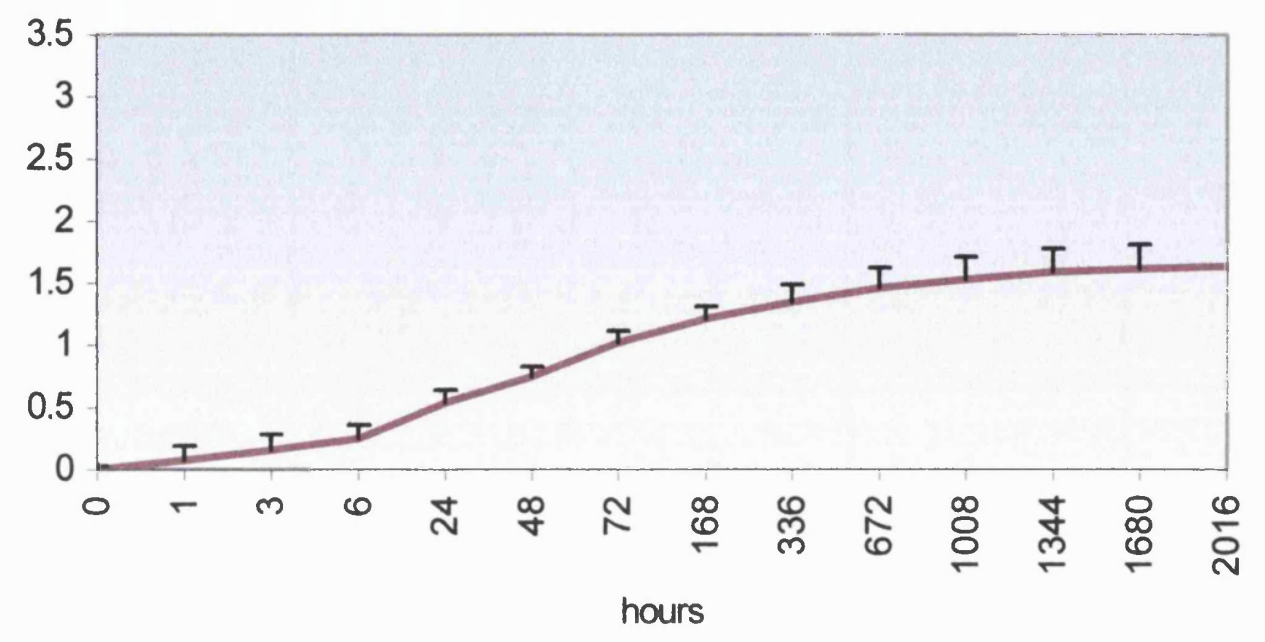

Figure 5.8 : Z100: Mean water sorption $\left(1^{\text {st }}\right.$ cycle $)$

The water sorption for Z100 during the first cycle was rapid and very similar to Silux Plus. By the end of one week nearly $75 \%$ of the total sorption had occurred. Equilibrium was achieved at around 6 weeks (1008 hours). This was similar to Silux Plus. The total water uptake was $1.64 \%$, much less than the $2.69 \%$ figure calculated for Silux Plus at the end of the first sorption cycle. The increase in the mass of $\mathrm{Z} 100$ during the first cycle was plotted against $\mathrm{t}^{1 / 2}$ (Figure 5.9). 


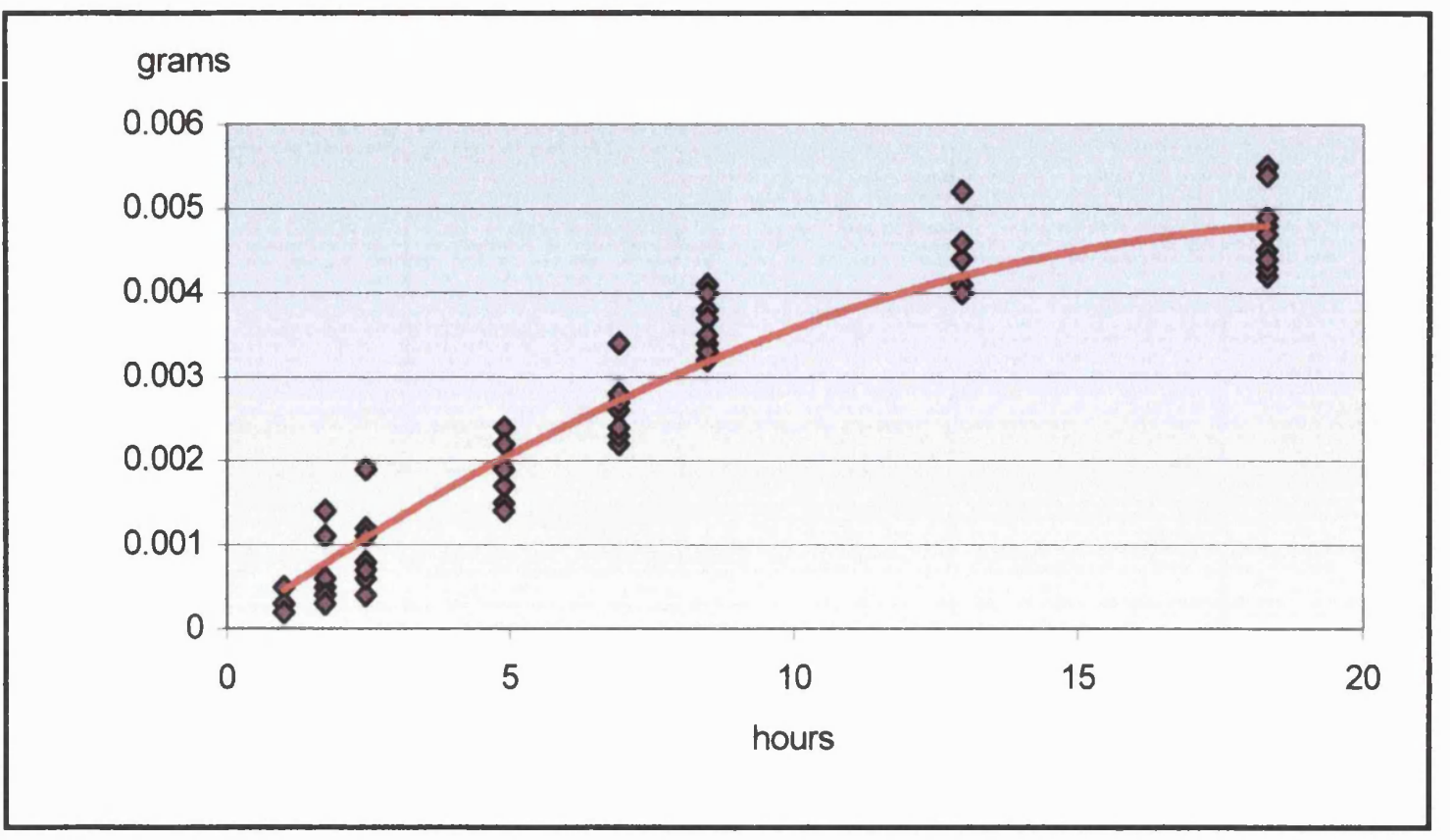

Figure $5.9: \mathrm{Z100}$ : Increase in mass with respect to $\mathrm{t}^{1 / 2}\left(1^{\text {st }}\right.$ cycle $)$

A scatter plot of the mass of each Z100 specimen during the second cycle is shown in Figure 5.10.

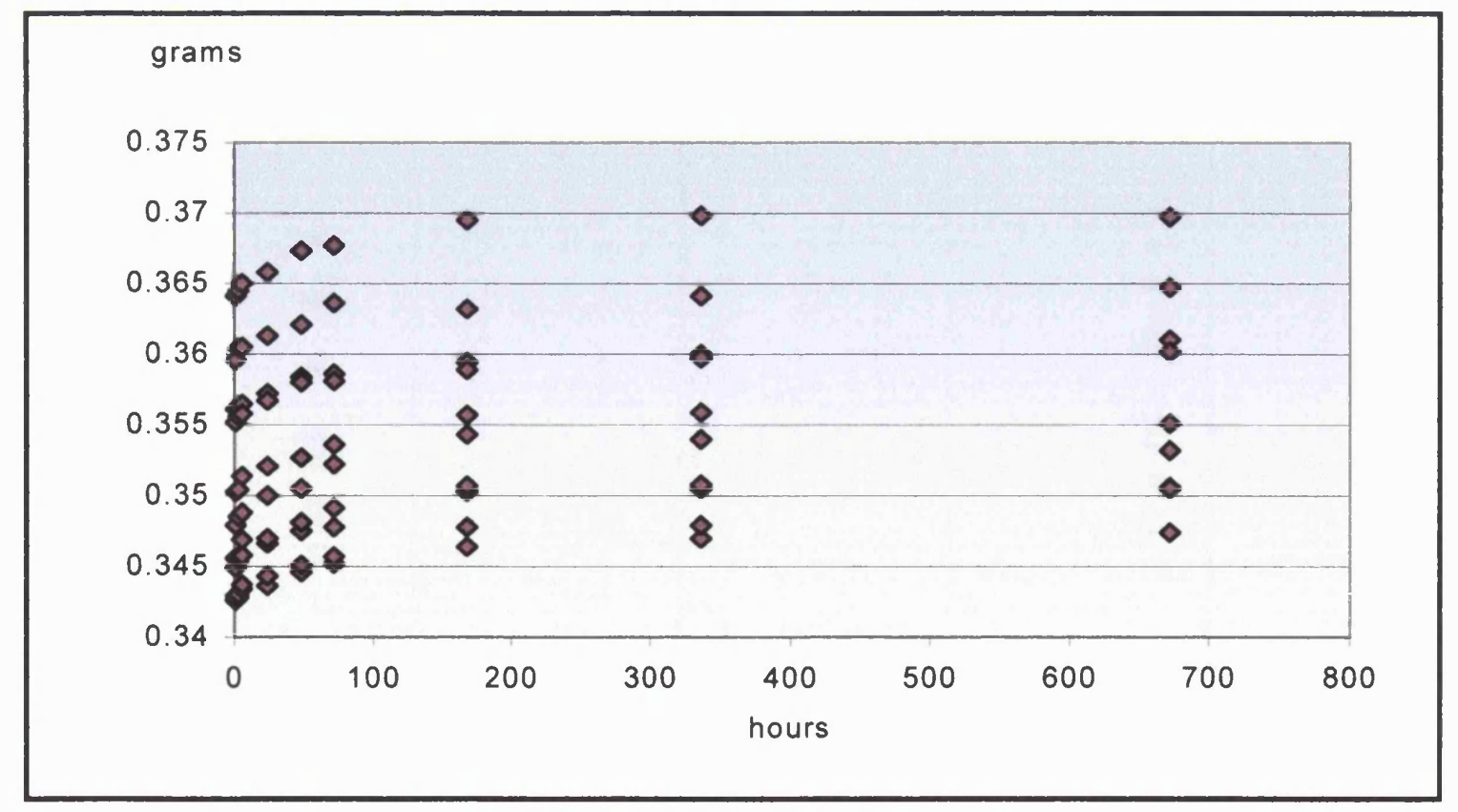

Figure 5.10 : Z100: Scatter distribution of individual specimens mass $\left(2^{\text {nd }}\right.$ cycle) 
The results for the second sorption cycle for Z100 are shown in Table 5.9 and Figure 5.11 .

\begin{tabular}{|l|c|c|c|c|c|c|c|}
\hline Hours & $\mathbf{0}$ & $\mathbf{1}$ & $\mathbf{3}$ & $\mathbf{6}$ & $\mathbf{2 4}$ & $\mathbf{4 8}$ & $\mathbf{7 2}$ \\
\hline Mean \%ws & 0 & 0.33 & 0.42 & 0.57 & 0.77 & 1.04 & 1.25 \\
S/d & 0.00 & 0.09 & 0.10 & 0.11 & 0.06 & 0.07 & 0.13 \\
\hline Hours & $\mathbf{1 6 8}$ & $\mathbf{3 3 6}$ & $\mathbf{6 7 2}$ & $\mathbf{1 0 0 8}$ & $\mathbf{1 3 4 4}$ & $\mathbf{1 6 8 0}$ & $\mathbf{2 0 1 6}$ \\
\hline Mean \%ws & 1.67 & 1.77 & 1.77 & 1.78 & 1.79 & 1.86 & 1.84 \\
S/d & 0.26 & 0.17 & 0.11 & 0.12 & 0.15 & 0.18 & 0.17 \\
\hline
\end{tabular}

Table 5.9 : Z100 water sorption (ws)

$\left(2^{\text {nd }}\right.$ cycle: Mean $\%$ values)

$\%$ water sorption

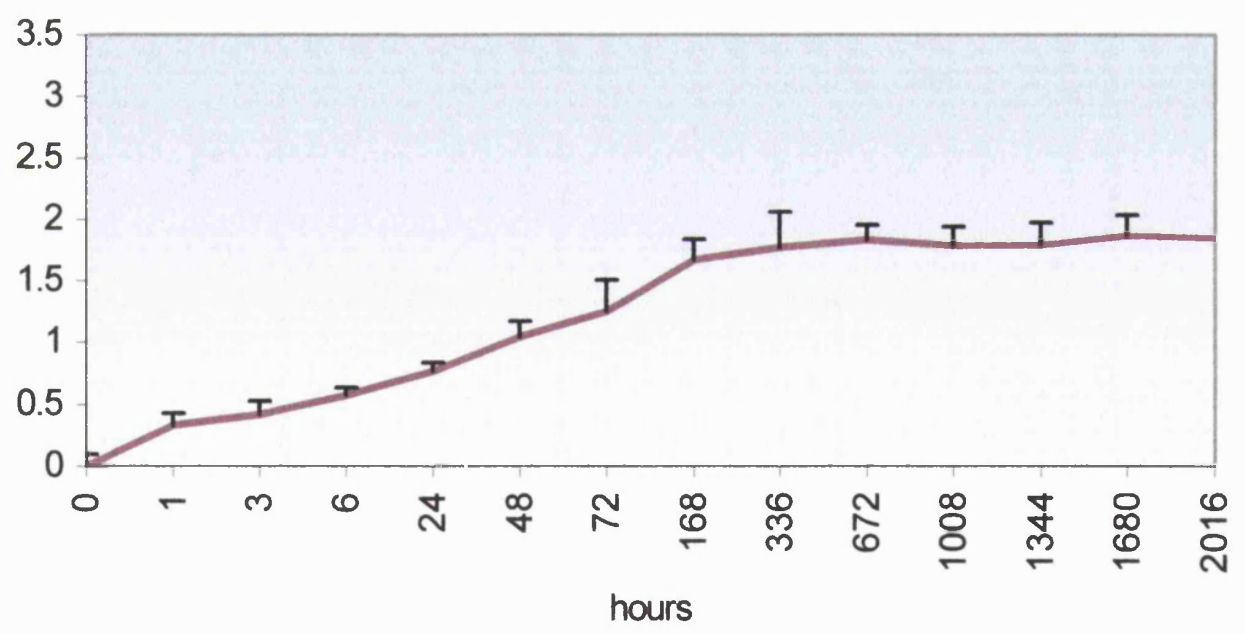

Figure $5.11:$ Z100: Mean water sorption $\left(2^{\text {nd }}\right.$ cycle $)$

The pattern observed in the second sorption cycle of Z100 was slightly different to the first cycle. Water sorption was rapid and equilibrium was achieved after the first four weeks ( 672 hours). Nearly $90 \%$ of the sorption, occurred by the end of the first week (168 hours). This was similar to Silux Plus.

Some variation was observed and the final value for sorption in this case was $1.84 \%$. 
However, the maximum sorption value of $\mathrm{Z} 100$ after the second cycle $(1.84 \%)$ is less than that observed for Silux Plus after its second sorption cycle $(2.88 \%)$. The increase in the mass of Z100 during the second cycle was plotted against $t^{1 / 2}$ (Figure 5.12).

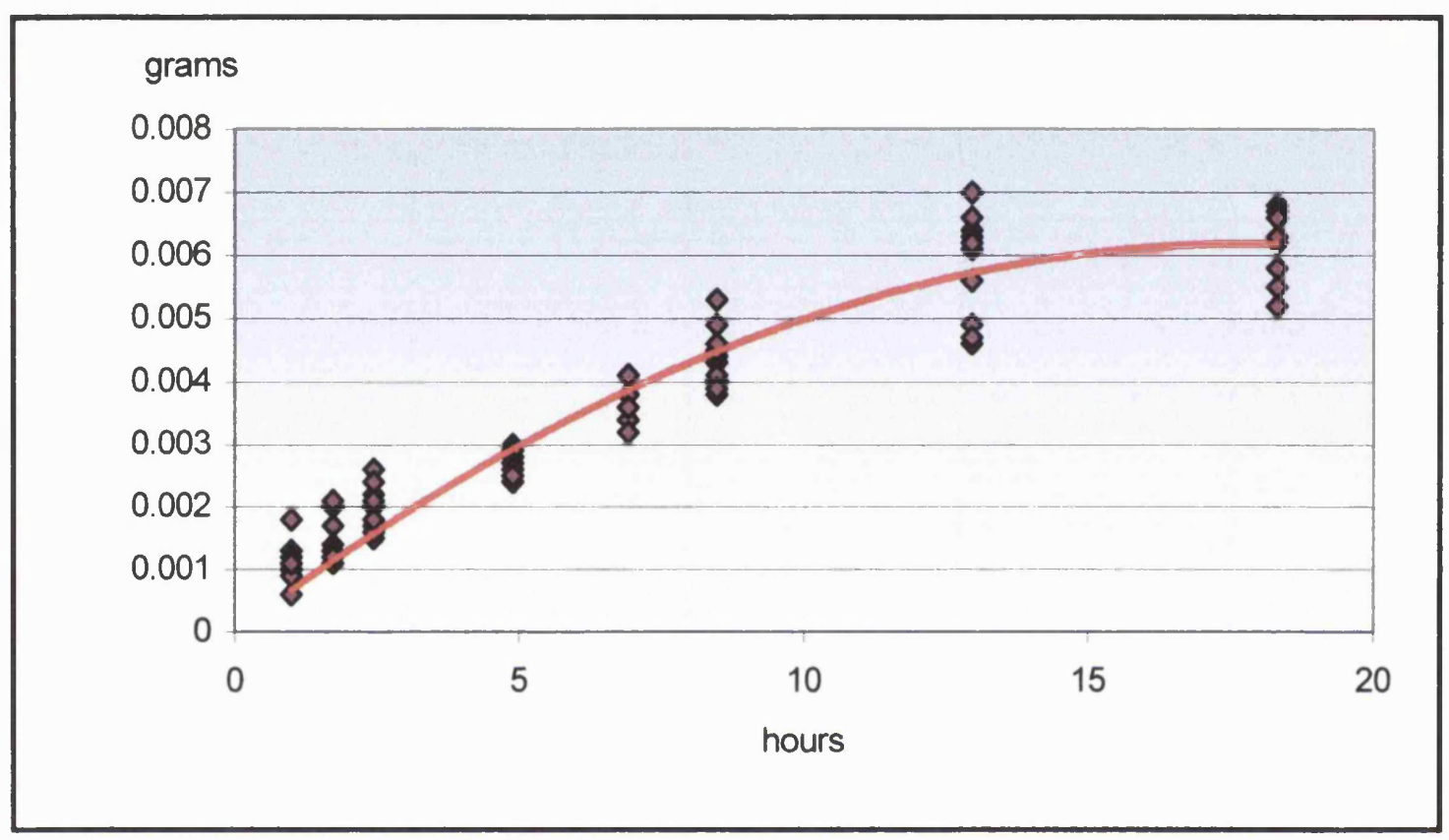

Figure $5.12: \mathrm{Z100:}$ Increase in mass with respect to $t^{1 / 2}\left(2^{\text {nd }}\right.$ cycle $)$

The calculated solubility value and standard deviation for Z100 is shown in Table 5.10.

\begin{tabular}{|l|c|}
\hline & Z100 \\
\hline Mean \% solubility & 0.19 \\
\hline S/d (\%) & 0.18 \\
\hline
\end{tabular}

Table 5.10 : Solubility (Z100 mean \% values)

There was a mean mass loss of $0.001 \mathrm{~g}$ between the start and the end of the first cycle. No further loss was observed at the second cycle. 


\subsubsection{COMPARATIVE WATER SORPTION IN BETWEEN MATERIALS}

The comparative figures of the percentage of water sorption between the two materials are listed in Table 5.11 and shown in Figures 5.13 and 5.14.

\begin{tabular}{|l|c|c|c|c|}
\hline Hours & $\begin{array}{c}\text { Silux Rlus } \\
\text { initial cycle } \\
\text { \% water } \\
\text { uptake }\end{array}$ & $\begin{array}{c}\text { Silux Plus } \\
\text { fimal cycle } \\
\text { \% water } \\
\text { uptake }\end{array}$ & $\begin{array}{c}\text { Z100 } \\
\text { initial cycle } \\
\text { \% water } \\
\text { uptake }\end{array}$ & $\begin{array}{c}\text { Z100 } \\
\text { final cycle } \\
\text { \% water } \\
\text { uptake }\end{array}$ \\
\hline 0 & 0 & 0 & 0 & 0 \\
1 & 0.20 & 0.36 & 0.07 & 0.33 \\
3 & 0.35 & 0.56 & 0.16 & 0.42 \\
6 & 0.70 & 0.78 & 0.25 & 0.57 \\
24 & 1.04 & 1.19 & 0.54 & 0.77 \\
48 & 1.45 & 1.61 & 0.75 & 1.04 \\
72 & 1.84 & 1.88 & 1.02 & 1.25 \\
168 & 2.00 & 2.57 & 1.21 & 1.67 \\
336 & 2.31 & 2.74 & 1.35 & 1.77 \\
672 & 2.45 & 2.85 & 1.46 & 1.83 \\
1008 & 2.58 & 2.80 & 1.53 & 1.78 \\
1344 & 2.65 & 2.80 & 1.59 & 1.79 \\
1680 & 2.68 & 2.87 & 1.61 & 1.86 \\
2016 & 2.69 & 2.88 & 1.64 & 1.84 \\
\hline
\end{tabular}

Table 5.11 : Comparison of mean \% water sorption for Silux Plus and Z100 in both sorption cycles 


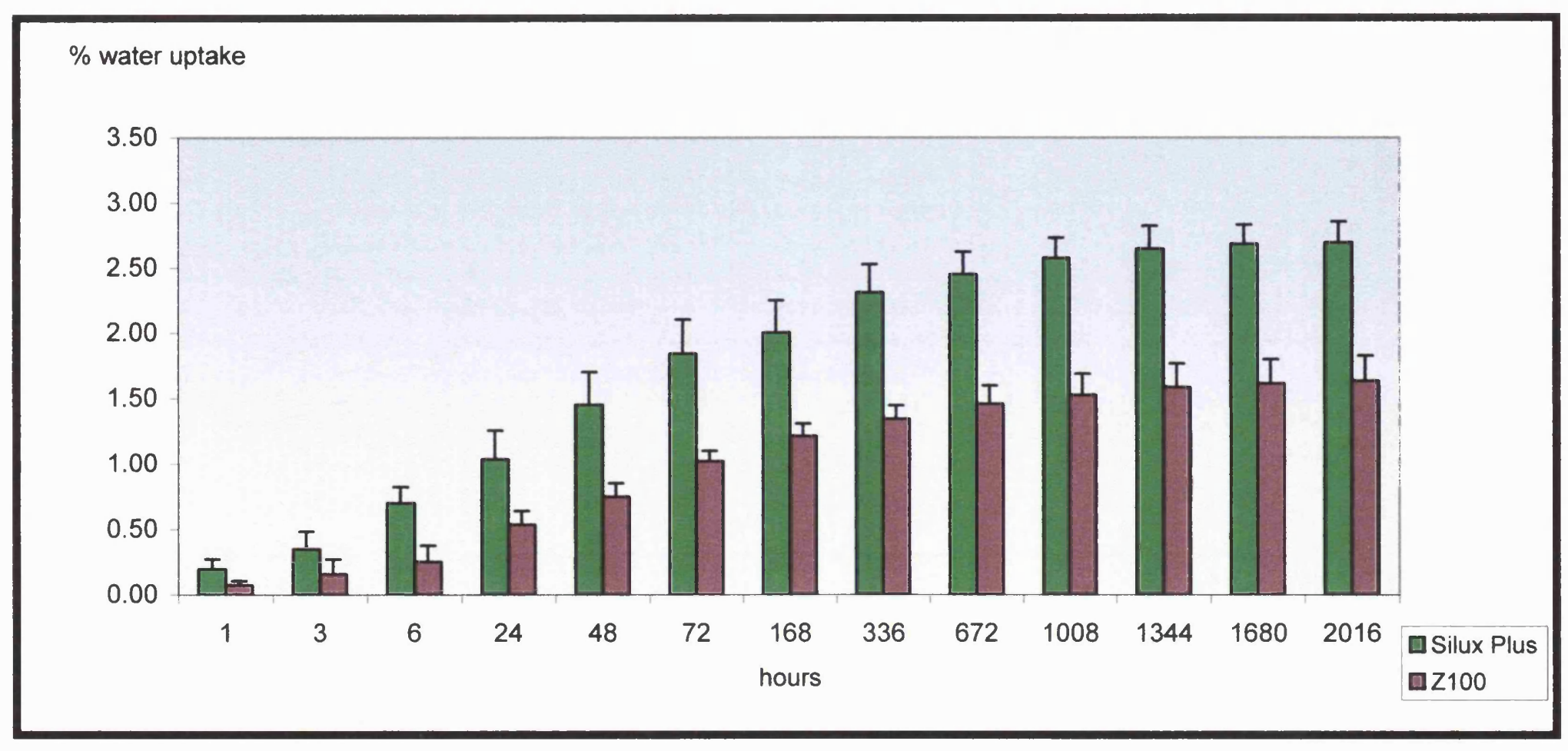

Figure 5.13 : Comparative \% water sorption for Silux Plus and Z100 ( $1^{\text {st }}$ cycle $)$ (Mean values) 


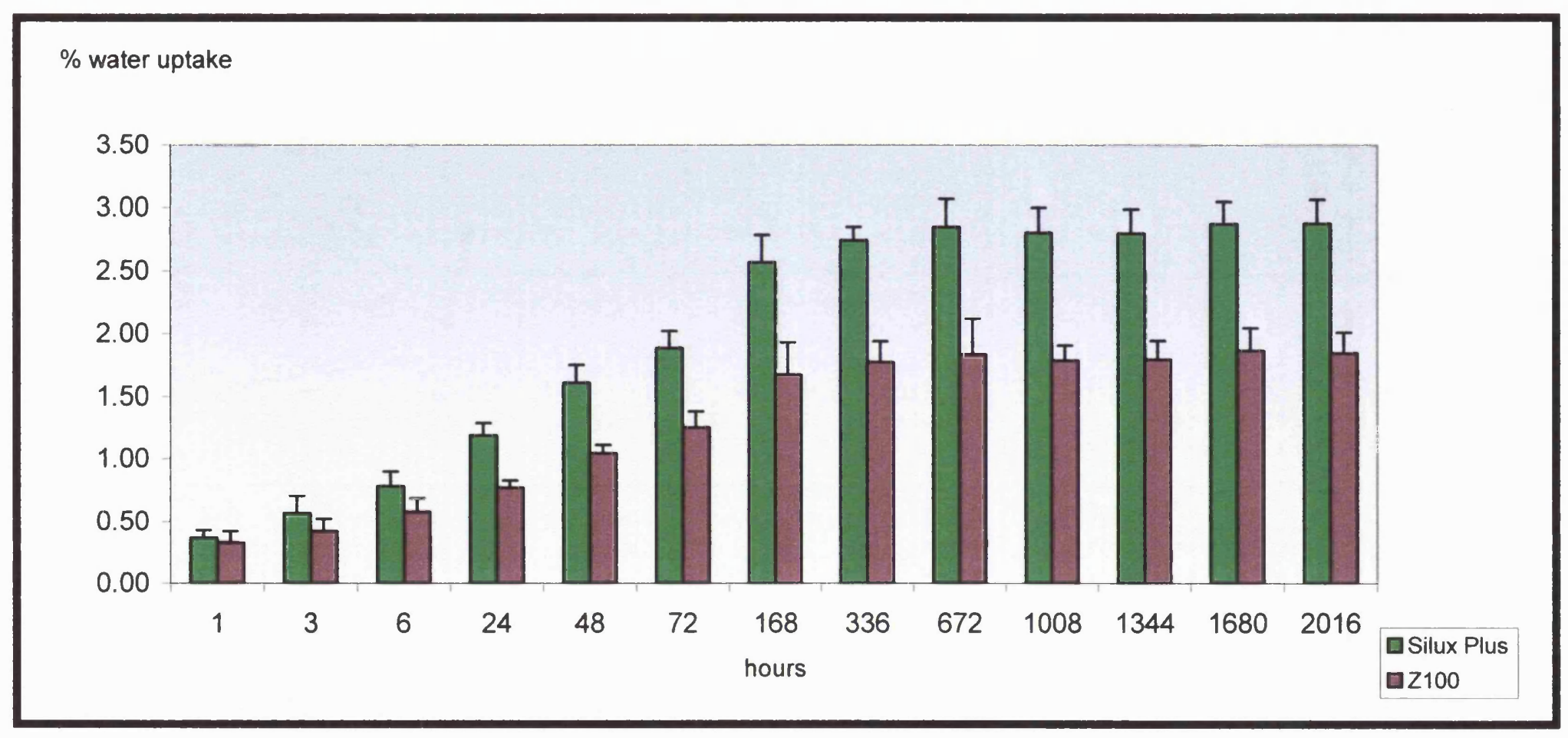

Figure 5.14 : Comparative \% water sorption for Silux Plus and Z100 ( $2^{\text {nd }}$ cycle) (Mean values) 


\subsubsection{4 pH VALUES OF STORAGE WATER}

$\mathrm{pH}$ values for the water prior to commencement and after immersion of the specimens for the test were also measured (Table 5.12).

\begin{tabular}{|l|c|}
\hline \multicolumn{1}{|c|}{ Material } & $\mathrm{pH}$ at 24 weeks \\
\hline Silux Plus & 6.98 \\
Z100 & 7.22 \\
Aged distilled $\mathrm{H}_{2} 0$ & 6.75 \\
\hline Fresh distilled $\mathrm{H}_{2} 0$ & 6.5 \\
\hline
\end{tabular}

Table 5.12 : Mean post test $\mathrm{pH}$ values of the water in which the specimens were stored 


\subsection{MAIN LOAD CYCLING AND THERMO-CYCLING STUDY}

\subsubsection{APPARENT FLEXURAL MODULUS}

The dimensions of the metal spine alone were entered into the software. The value obtained reflected an apparent flexural modulus for the metal/resin structure since the resins themselves were not loaded directly, but just modified the properties of the metal spine.

\subsubsection{SILUX PLUS}

\subsection{LOAD CYCLED GROUP}

The apparent mean flexural modulus for Silux Plus bonded resin to metal specimens tested immediately after preparation was $288.1( \pm 44.4) \mathrm{GPa}$. The apparent flexural moduli for the Silux Plus specimens subjected to load cycling alone at different time intervals are shown in Table 5.13 and Figure 5.15. Intergroup comparisons were carried out using the Mann-Whitney test and the statistically significant differences between groups are recorded in Table 5.14.

Specimens were load cycled for periods up to the equivalent of 24 weeks. Initially the values for the apparent modulus increased steadily up to week $8(353.5( \pm 43.4) \mathrm{GPa})$. Subsequently, the values began to decline. By week 12 , the value (292.7 ( \pm 55.7$) \mathrm{GPa})$ had fallen to $82 \%$ of that noted after 8 weeks load cycling. Thereafter, the values remained relatively unchanged up to week 20 but by week 24 a gradual increase in value was noted, the 24 week value being similar to that at 8 weeks. 


\begin{tabular}{|l|c|c|c|c|c|c|c|c|c|}
\hline Time (Weeks) & $\mathbf{0}$ & $\mathbf{1}$ & $\mathbf{2}$ & $\mathbf{4}$ & $\mathbf{8}$ & $\mathbf{1 2}$ & $\mathbf{1 6}$ & $\mathbf{2 0}$ & $\mathbf{2 4}$ \\
\hline Mean $(\mathrm{GPa})$ & 288.1 & 298.5 & 314.7 & 326.7 & 353.5 & 292.7 & 303.4 & 322.1 & 350.8 \\
S/d & 44.4 & 92.4 & 33.8 & 41.2 & 43.4 & 55.7 & 38.8 & 29.8 & 38 \\
Median (GPa) & 291 & 306.8 & 319.7 & 325.8 & 342.3 & 285.3 & 294.3 & 323.3 & 356.7 \\
\hline
\end{tabular}

Table 5.13 : Apparent Flexural Modulus of Load Cycled Silux Plus (GPa)

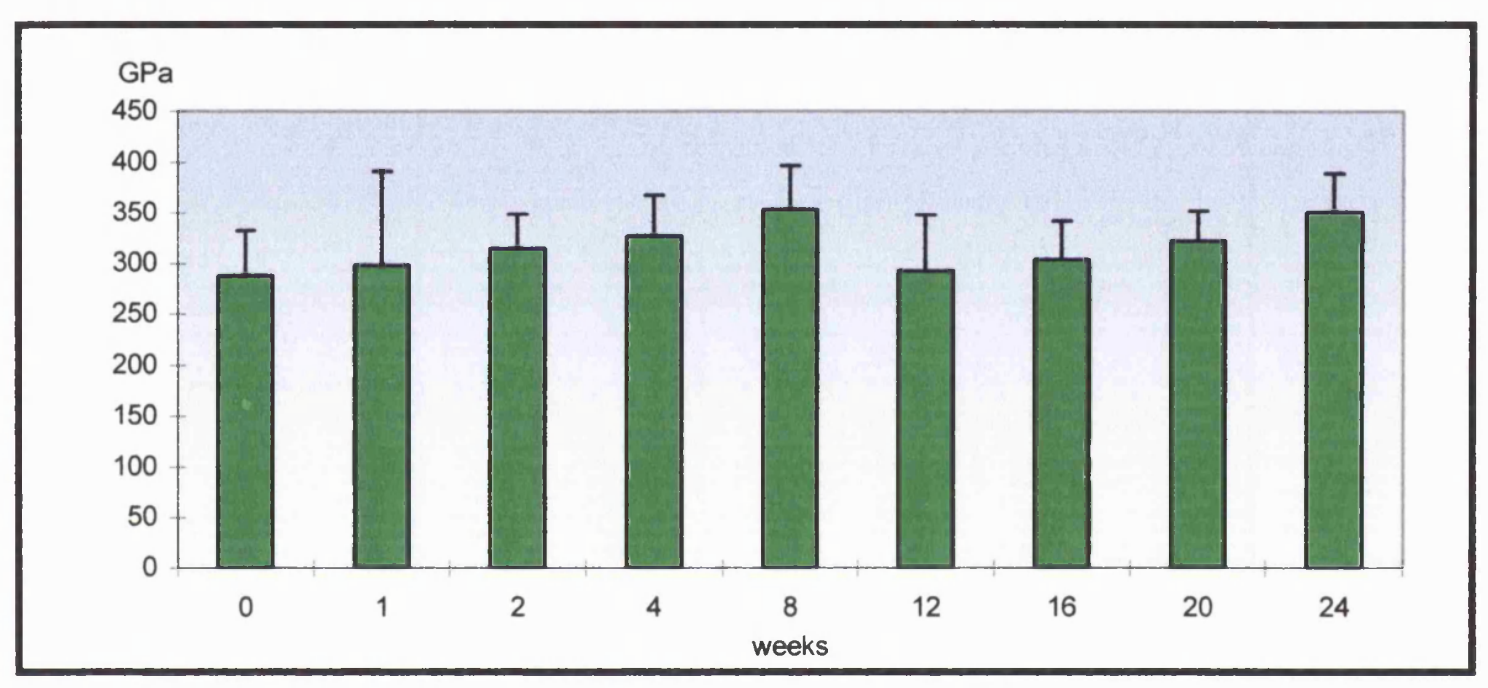

Figure 5.15 : Apparent Flexural Modulus of Load Cycled Silux Plus (GPa) (Mean values)

\begin{tabular}{|l|c|c|c|c|}
\hline Week & 0 & 8 & 12 & 16 \\
\hline 8 & H/S & & & \\
\hline 12 & & S & & \\
\hline 16 & & S & & \\
\hline 24 & H/S & & S & S \\
\hline
\end{tabular}

$\mathrm{H} / \mathrm{S}<0.01$

$\mathrm{S}<0.05$

Table 5.14 : Statistical Analysis of Apparent Flexural Modulus for Load Cycled Silux Plus 


\subsection{THERMO-CYCLED GROUP}

The apparent flexural modulus for the Silux Plus specimens subjected to thermo-cycling alone is shown in Table 5.15 and Figure 5.16. Intergroup comparisons were carried out using the Mann-Whitney test and the statistically significant differences between groups are recorded in Table 5.16.

There was no statistical difference between samples within the first two weeks. Thereafter, a significant increase in strength was observed. After 8 weeks, a $17 \%$ decline in the apparent modulus values was observed. By week 24 this decline had been reversed.

A comparison of the load cycled with the thermo-cycled groups showed there was no significant difference in the apparent modulus at week 8,12 and 24 , between the different treatments. 


\begin{tabular}{|l|c|c|c|c|c|c|c|c|c|}
\hline Time (Weeks) & $\mathbf{0}$ & $\mathbf{1}$ & $\mathbf{2}$ & $\mathbf{4}$ & $\mathbf{8}$ & $\mathbf{1 2}$ & $\mathbf{1 6}$ & $\mathbf{2 0}$ & $\mathbf{2 4}$ \\
\hline Mean (GPa) & 288.1 & 283.6 & 323.3 & 343.8 & 348.4 & 290.8 & 299 & 302.2 & 367 \\
S/d & 44.4 & 46.7 & 29.8 & 30.7 & 78.7 & 79.5 & 29.1 & 52.6 & 35.6 \\
Median (GPa) & 291 & 296.5 & 320 & 347.4 & 348.6 & 318.5 & 302.5 & 303.1 & 379.5 \\
\hline
\end{tabular}

Table 5.15 : Apparent Flexural Modulus of Thermo-Cycled Silux Plus (GPa)

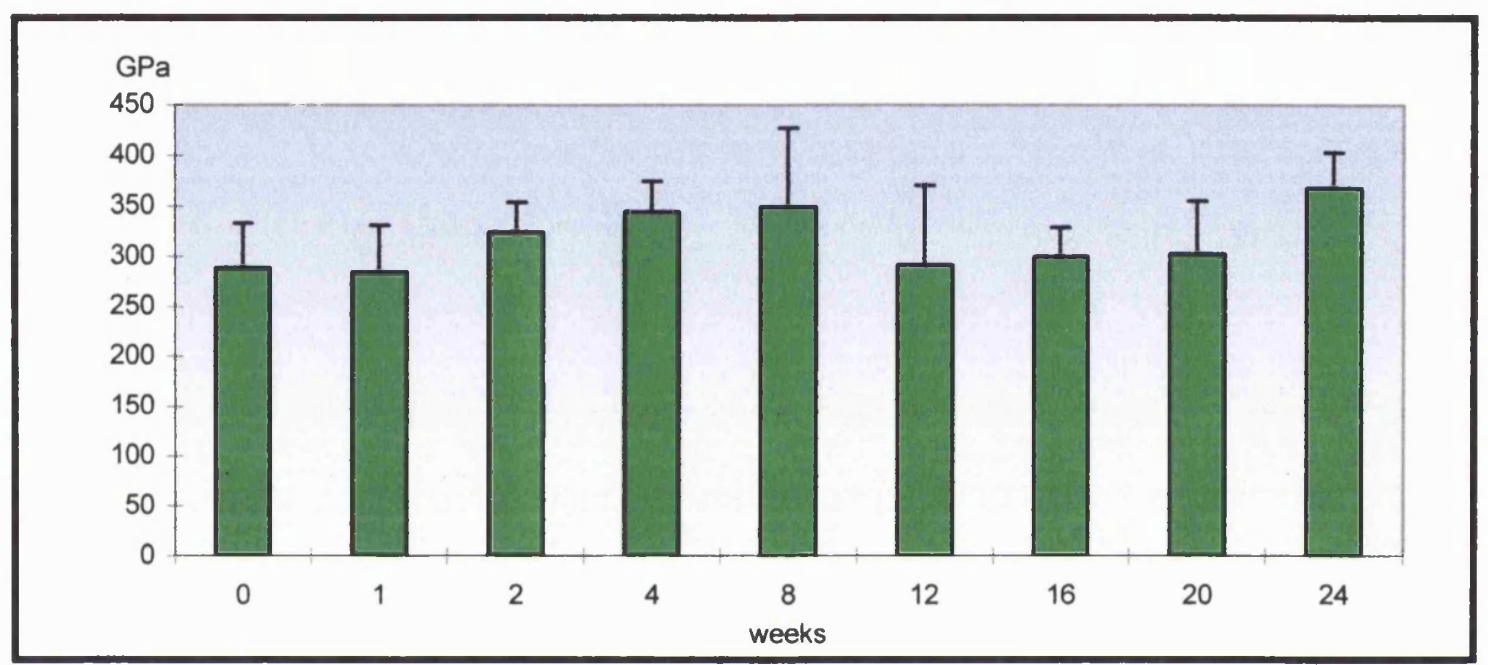

Figure 5.16 : Apparent Flexural Modulus of Thermo-Cycled Silux Plus (GPa) (Mean values)

\begin{tabular}{|l|c|c|c|c|c|c|c|}
\hline Week & 0 & 1 & 2 & 4 & 12 & 16 & 24 \\
\hline 4 & H/S & H/S & & & & & \\
\hline 16 & & & & H/S & & & \\
\hline 20 & & & & S & & & S \\
\hline 24 & H/S & H/S & H/S & S & H/S & H/S & \\
\hline
\end{tabular}

$$
\begin{array}{r}
\mathrm{H} / \mathrm{S}<0.01 \\
\mathrm{~S}<0.05
\end{array}
$$

Table 5.16 : Statistical Analysis of Apparent Flexural Modulus for Thermo-Cycled Silux Plus 


\subsection{THERMO/LOAD CYCLED GROUP}

The apparent flexural modulus for the Silux Plus specimens subjected to both thermo and load cycling is shown in Table 5.17 and Figure 5.17. Intergroup comparisons were carried out using the Mann-Whitney test and the statistically significant differences between groups are recorded in Table 5.18. In the combined thermo and load cycled specimens, a similar pattern was observed when compared to the previous results for the specimens subjected to thermo or load cycling alone. However, the increase in the apparent flexural modulus of the specimens was less apparent up to week 8. Similarly, the reduction in modulus between week 8 and week 12 was only $8 \%$. This is substantially less than the values observed in the specimens of the groups that they were either thermo or load cycled alone. By week 24 , the value for the apparent flexural modulus was $20 \%$ more than the value obtained immediately after manufacture.

\subsection{COMPARISON OF THE DIFFERENT SILUX PLUS CYCLING GROUPS}

Inter group comparison of the apparent flexural modulus results for Silux Plus is shown in Figure 5.18. Inter group comparisons in between the different treatments at similar time intervals showed no statistically significant differences. 


\begin{tabular}{|l|c|c|c|c|c|c|c|c|c|}
\hline Time (Weeks) & $\mathbf{0}$ & $\mathbf{1}$ & $\mathbf{2}$ & $\mathbf{4}$ & $\mathbf{8}$ & $\mathbf{1 2}$ & $\mathbf{1 6}$ & $\mathbf{2 0}$ & $\mathbf{2 4}$ \\
\hline Mean (GPa) & 288.1 & 255.4 & 309.9 & 321 & 332.6 & 308.2 & 314.5 & 303.8 & 353.7 \\
S/d & 44.4 & 93.6 & 44.5 & 43.4 & 54.2 & 19.7 & 29.1 & 22.4 & 47.5 \\
Median (GPa) & 291 & 274.1 & 322.3 & 316.1 & 333.6 & 306.2 & 315.5 & 304.4 & 345.4 \\
\hline
\end{tabular}

Table 5.17 : Apparent Flexural Modulus of Thermo/Load Cycled Silux Plus (GPa)

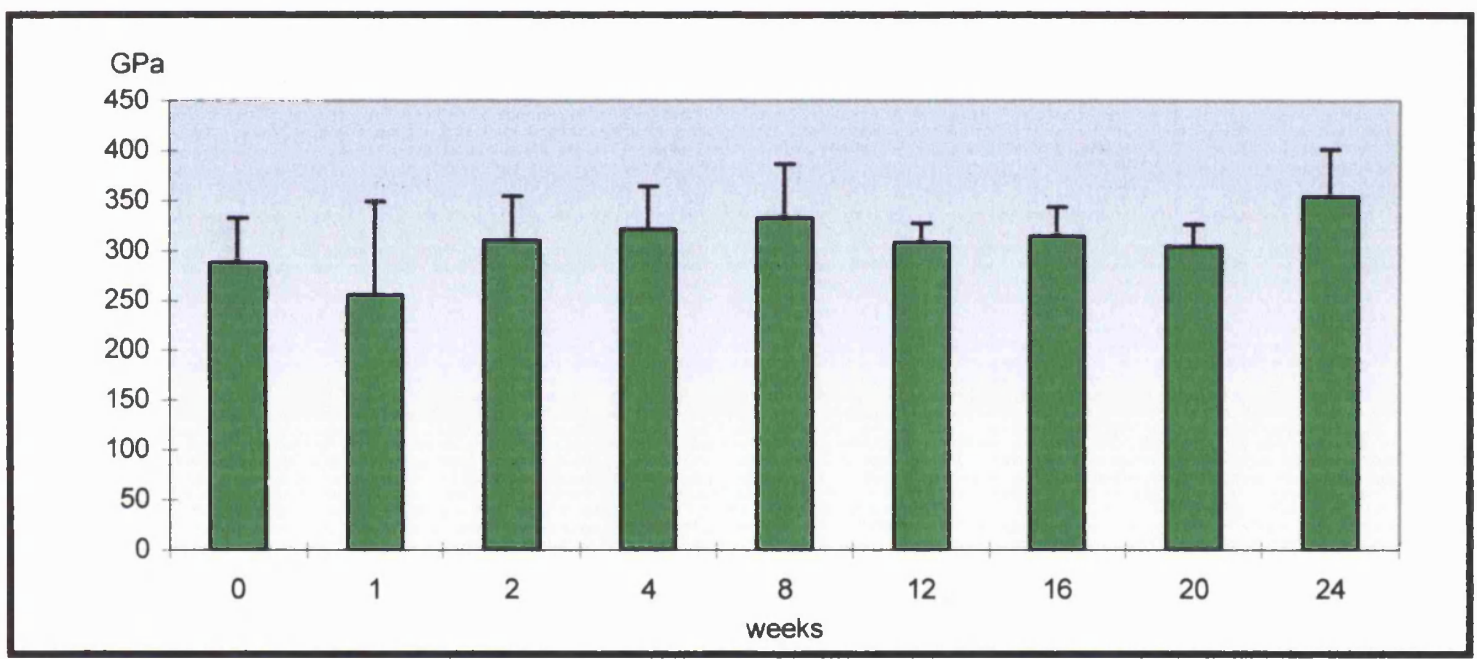

Figure 5.17 : Apparent Flexural Modulus of Thermo/Load Cycled Silux Plus (GPa) (Mean values)

\begin{tabular}{|l|c|c|c|c|}
\hline Week & 0 & 1 & 12 & 20 \\
\hline 24 & $\mathrm{~S}$ & $\mathrm{~S}$ & $\mathrm{~S}$ & $\mathrm{~S}$ \\
\hline
\end{tabular}

$\mathrm{H} / \mathrm{S}<0.01$

Table 5.18 : Statistical Analysis of Apparent Flexural Modulus for Thermo/Load Cycled Silux Plus 


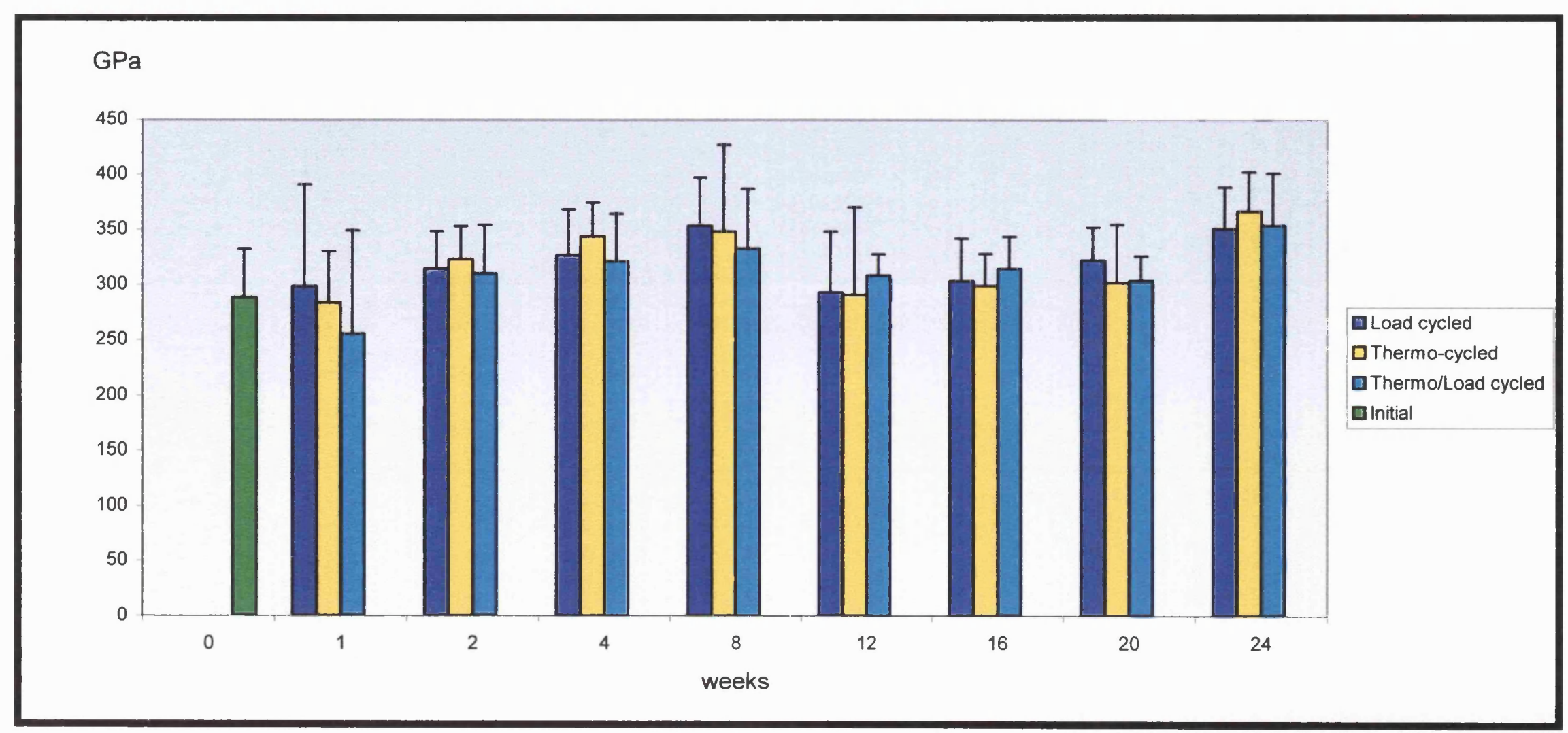

Figure 5.18 : Apparent Flexural Modulus for the different Silux Plus testing groups (GPa) (Mean values) 


\subsubsection{Z100}

\subsection{LOAD CYCLED GROUP}

The mean apparent flexural modulus for the bonded $\mathrm{Z} 100$ resin to metal specimens tested immediately after preparation was $482.3( \pm 69.1) \mathrm{GPa}$. The apparent flexural modulus for the Z100 specimens subjected to load cycling alone is shown in Table 5.19 and Figure 5.19. As before, intergroup comparisons were carried out using the Mann-Whitney test and the statistically significant differences between groups are recorded in Table 5.20. As the specimens were load cycled, the values for the apparent flexural modulus appeared to remain stable up to week $12(502.7( \pm 56.8) \mathrm{GPa})$. After this period the values fell significantly to $80 \%$ of the week 12 value $(393.5( \pm 125.4) \mathrm{GPa})$. The decrease is similar to that observed for Silux Plus, however Silux Plus exhibited a decline at an earlier stage. From week 16 to week 24 the modulus value increased by $35 \%$ for the Z100 load cycled specimens. At the end of week 24, the value of the apparent flexural modulus was $20 \%$ higher than the initial recorded value. 


\begin{tabular}{|l|c|c|c|c|c|c|c|c|c|}
\hline Time (Weeks) & $\mathbf{0}$ & $\mathbf{1}$ & $\mathbf{2}$ & $\mathbf{4}$ & $\mathbf{8}$ & $\mathbf{1 2}$ & $\mathbf{1 6}$ & $\mathbf{2 0}$ & $\mathbf{2 4}$ \\
\hline Mean (GPa) & 482.3 & 485.2 & 531.9 & 547.6 & 489 & 502.7 & 393.5 & 443.4 & 606.1 \\
S/d & 69.1 & 73.3 & 76.7 & 30.5 & 136.4 & 56.8 & 125.4 & 183.8 & 50.2 \\
Median (GPa) & 491 & 501.6 & 54.9 & 540.4 & 526.9 & 515.1 & 433.5 & 495.6 & 600.3 \\
\hline
\end{tabular}

Table 5.19 : Apparent Flexural Modulus of Load Cycled Z100 (GPa)

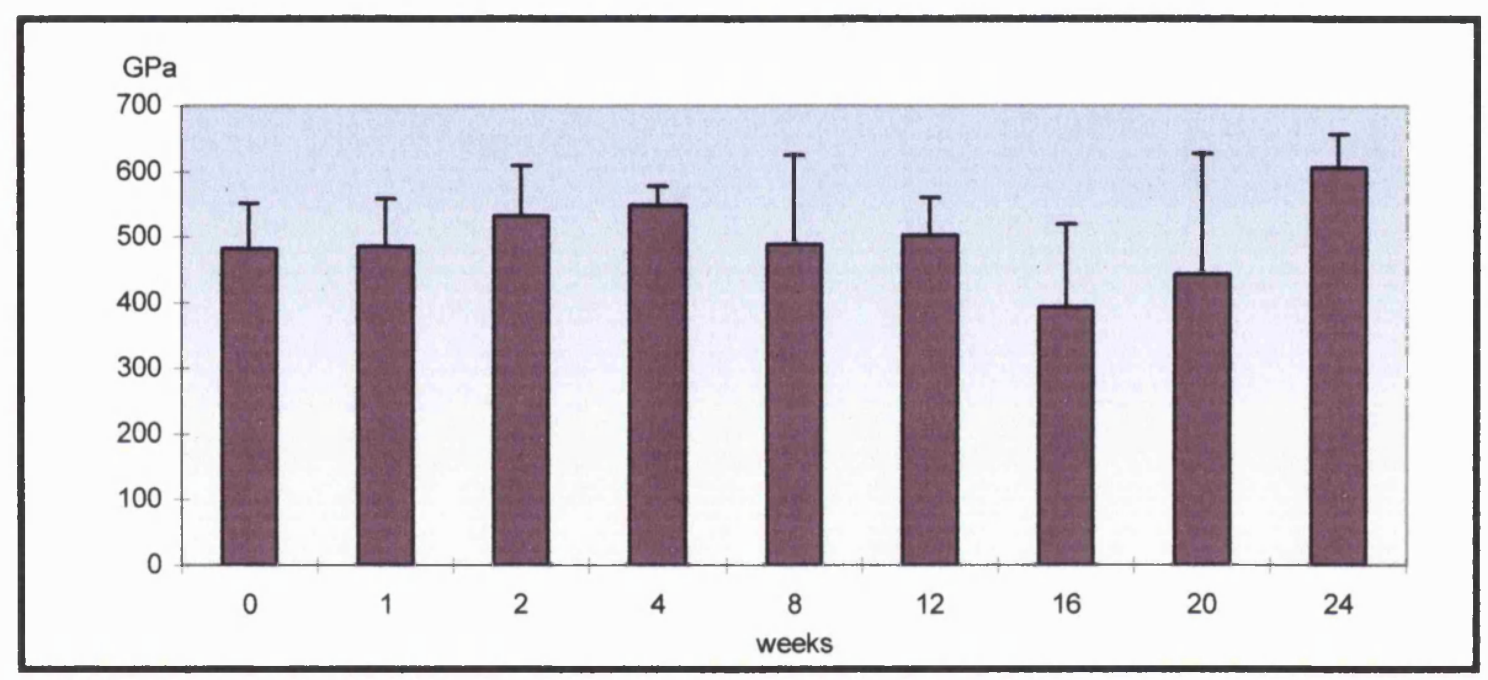

Figure 5.19 : Apparent Flexural Modulus of Load Cycled Z100 (GPa) (Mean values)

\begin{tabular}{|l|c|c|c|c|c|c|c|c|}
\hline Week & 1 & 2 & 4 & 8 & 12 & 16 & 20 & 24 \\
\hline 0 & & & & & & & & H/S \\
\hline 16 & & H/S & H/S & & S & & & \\
\hline 24 & H/S & S & S & S & H/S & H/S & H/S & \\
\hline
\end{tabular}

$$
\begin{array}{r}
\mathrm{H} / \mathrm{S}<0.01 \\
\mathrm{~S}<0.05
\end{array}
$$

Table 5.20 : Statistical Analysis of Apparent Flexural Modulus for Load Cycled Z100 


\subsection{THERMO-CYCLED GROUP}

The apparent flexural modulus for the Z100 specimens subjected to thermo-cycling alone is shown in Table 5.21 and Figure 5.20. Intergroup comparisons were carried out using the Mann-Whitney test and the statistically significant differences between groups are recorded in Table 5.22. With the specimens which were subjected to thermo-cycling alone, there was a statistically significant increase $(20 \%)$ in the value of the apparent flexural modulus during the first 2 weeks $(607.8( \pm 77.8) \mathrm{GPa})$, over the initial value recorded $(482.3( \pm 69.1) \mathrm{GPa})$. Thereafter, there was a trend to reduce the apparent flexural modulus up to week 24.

This was similar to the effect observed with load cycling alone. In the case of thermocycling there was a $17 \%$ reduction in the apparent flexural modulus after week 12 . Thereafter the values tended to increase until week 24 when they showed a $15 \%$ increase to the value of week 16 . This was still above the initial value obtained prior to cycling. 


\begin{tabular}{|l|c|c|c|c|c|c|c|c|c|}
\hline Time (Weeks) & $\mathbf{0}$ & $\mathbf{1}$ & $\mathbf{2}$ & $\mathbf{4}$ & $\mathbf{8}$ & $\mathbf{1 2}$ & $\mathbf{1 6}$ & $\mathbf{2 0}$ & $\mathbf{2 4}$ \\
\hline Mean (GPa) & 482.3 & 453.3 & 607.8 & 504.7 & 516.9 & 502.9 & 420.5 & 459.3 & 556.5 \\
S/d & 69.1 & 86.6 & 77.8 & 86.4 & 51.9 & 59.2 & 73.8 & 47.5 & 67.1 \\
Median (GPa) & 491 & 452.8 & 605.4 & 512.2 & 523.6 & 492.5 & 417 & 452 & 539.7 \\
\hline
\end{tabular}

Table 5.21 : Apparent Flexural Modulus of Thermo-Cycled Z100 (GPa)

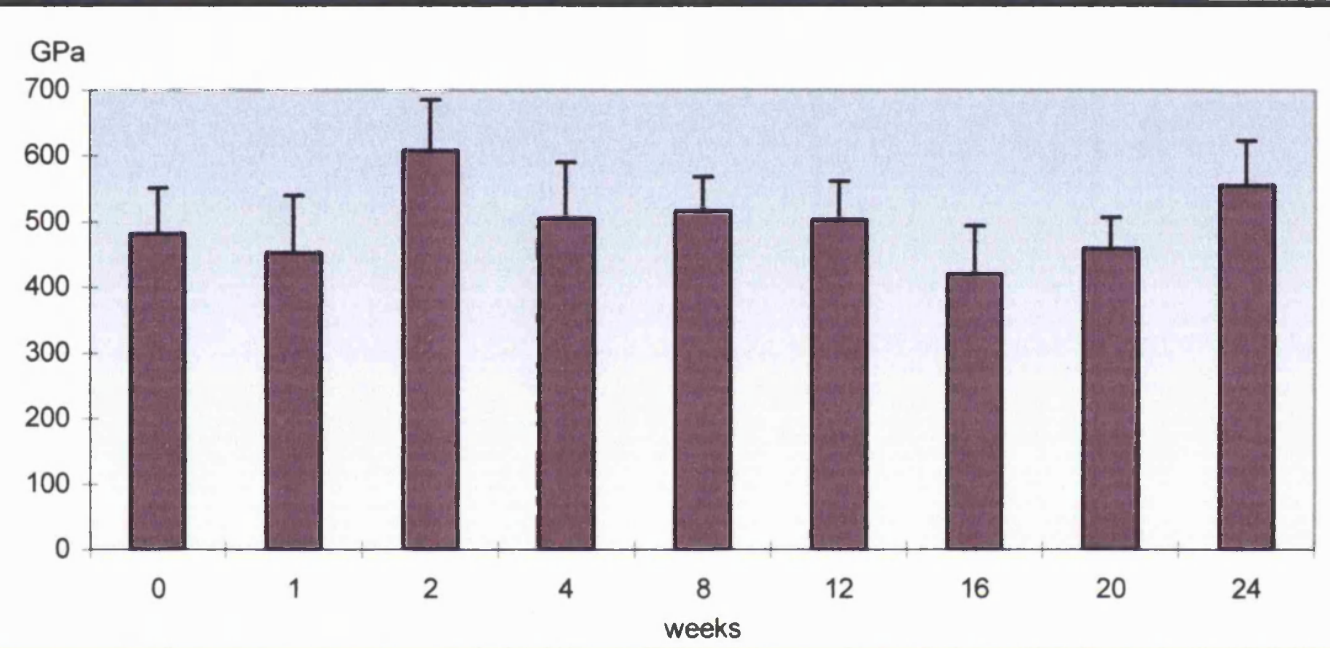

Figure 5.20 : Apparent Flexural Modulus of Thermo-Cycled Z100 (GPa) (Mean values)

\begin{tabular}{|l|c|c|c|c|c|c|c|c|}
\hline Week & 0 & 2 & 4 & 8 & 12 & 16 & 20 & 24 \\
\hline 1 & & H/S & & & & & & S \\
\hline 2 & $\mathrm{~S}$ & & $\mathrm{~S}$ & $\mathrm{~S}$ & $\mathrm{H} / \mathrm{S}$ & $\mathrm{H} / \mathrm{S}$ & $\mathrm{H} / \mathrm{S}$ & \\
\hline 4 & & & & & & $\mathrm{~S}$ & & \\
\hline 8 & & & & & & $\mathrm{H} / \mathrm{S}$ & $\mathrm{S}$ & \\
\hline 12 & & & & & & $\mathrm{H} / \mathrm{S}$ & & \\
\hline 16 & & & & & & & & $\mathrm{H} / \mathrm{S}$ \\
\hline 20 & & & & & & & & $\mathrm{H} / \mathrm{S}$ \\
\hline
\end{tabular}

$\mathrm{H} / \mathrm{S}<0.01$

$\mathrm{S}<0.05$

Table 5.22 : Statistical Analysis of Apparent Flexural Modulus for Thermo-Cycled Z100 


\subsection{THERMO/LOAD CYCLED GROUP}

The apparent flexural modulus for the Z100 specimens subjected to both thermo and load cycling is shown in Table 5.23 and Figure 5.21. Intergroup comparisons were carried out using the Mann-Whitney test and the statistically significant differences between groups are recorded in Table 5.24. A similar pattern was observed with the combined thermo and load cycled specimens to that noted for the specimens that they were subjected to either thermo or load cycling alone. In fact, the changes observed with the combined load and thermo-cycled group were less pronounced. This was similar to the results observed when Silux Plus was used as the composite spine material.

The reduction in the strength of the Z100 specimens between week 12 and week 16 was $16 \%$, similar to the corresponding percentage reduction noted for the individual groups. The dual cycling did not appear to produce a greater reduction when compared to either load or thermo-cycling. Z100 specimens showed an increase in value and by week 24 , there was a $33 \%$ increase in the value of the apparent flexural modulus. 


\begin{tabular}{|l|c|c|c|c|c|c|c|c|c|}
\hline Time (Weeks) & $\mathbf{0}$ & $\mathbf{1}$ & $\mathbf{2}$ & $\mathbf{4}$ & $\mathbf{8}$ & $\mathbf{1 2}$ & $\mathbf{1 6}$ & $\mathbf{2 0}$ & $\mathbf{2 4}$ \\
\hline Mean (GPa) & 482.3 & 537.9 & 498.6 & 494.1 & 421.8 & 434.4 & 367.3 & 416.3 & 544.7 \\
S/d & 69.1 & 96.3 & 59.5 & 112.3 & 170.7 & 163 & 188.1 & 159.3 & 70.3 \\
Median (GPa) & 491 & 550.6 & 480.5 & 509.6 & 486.2 & 439.2 & 389.9 & 450 & 544.9 \\
\hline
\end{tabular}

Table 5.23 : Apparent Flexural Modulus of Thermo/Load Cycled Z100 (GPa)

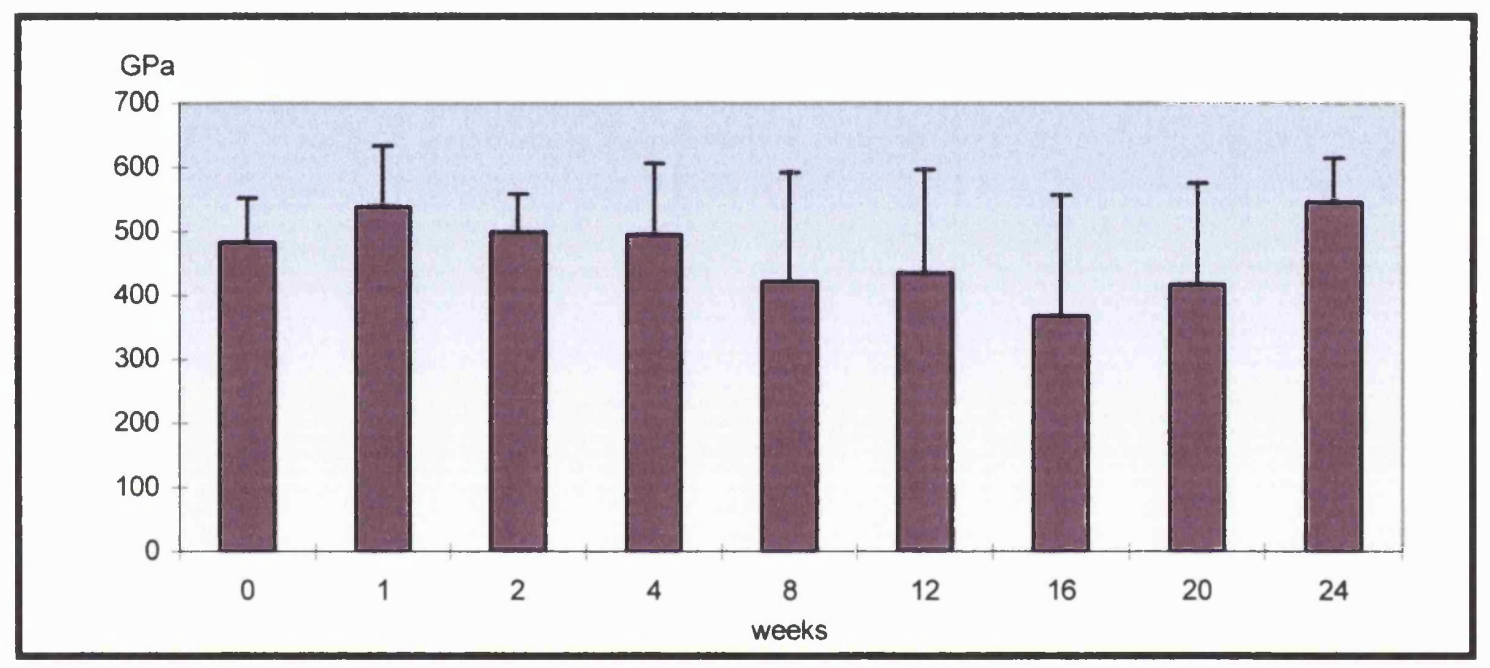

Figure 5.21 : Apparent Flexural Modulus of Thermo/Load Cycled Z100 (GPa) (Mean values)

\begin{tabular}{|l|c|}
\hline Week & 24 \\
\hline 16 & S \\
\hline 20 & S \\
\hline
\end{tabular}

$$
\begin{array}{r}
\mathrm{H} / \mathrm{S}<0.01 \\
\mathrm{~S}<0.05
\end{array}
$$

Table 5.24 : Statistical Analysis of Apparent Flexural Modulus for Thermo/Load Cycled Z100 


\subsection{COMPARISON OF THE DIFFERENT Z100 CYCLING GROUPS}

Inter group comparison of the apparent flexural modulus results for $\mathrm{Z} 100$ is shown in Figure 5.22. Intergroup comparisons were carried out between treatments at similar time intervals using the Mann-Whitney test and the statistically significant differences between groups are recorded in Table 5.25. Of these, there was a highly significantly difference in between week 2 thermo-cycled group when compared with the lower value of the combined thermo/load cycled group. Also, there was a significant difference in between week 24 load cycled group and the lower value of the combined thermo and load cycled group.

\begin{tabular}{|c|c|c|c|}
\hline Week & Group & $\begin{array}{c}\text { Thermo/Load } \\
\text { cycled }\end{array}$ & $\begin{array}{c}\text { Thermo/Load } \\
\text { cycled }\end{array}$ \\
\hline 2 & $\begin{array}{c}\text { Thermo- } \\
\text { cycled }\end{array}$ & H/S & \\
\hline 24 & $\begin{array}{c}\text { Load } \\
\text { cycled }\end{array}$ & $\mathbf{S}$ \\
\hline \multicolumn{3}{|c|}{$\begin{array}{c}\mathrm{H} / \mathrm{S}<0.01 \\
\mathrm{~S}<0.05\end{array}$} \\
\hline
\end{tabular}

Table 5.25 : Statistical Analysis of Apparent Flexural Modulus for the different Z100 testing groups 


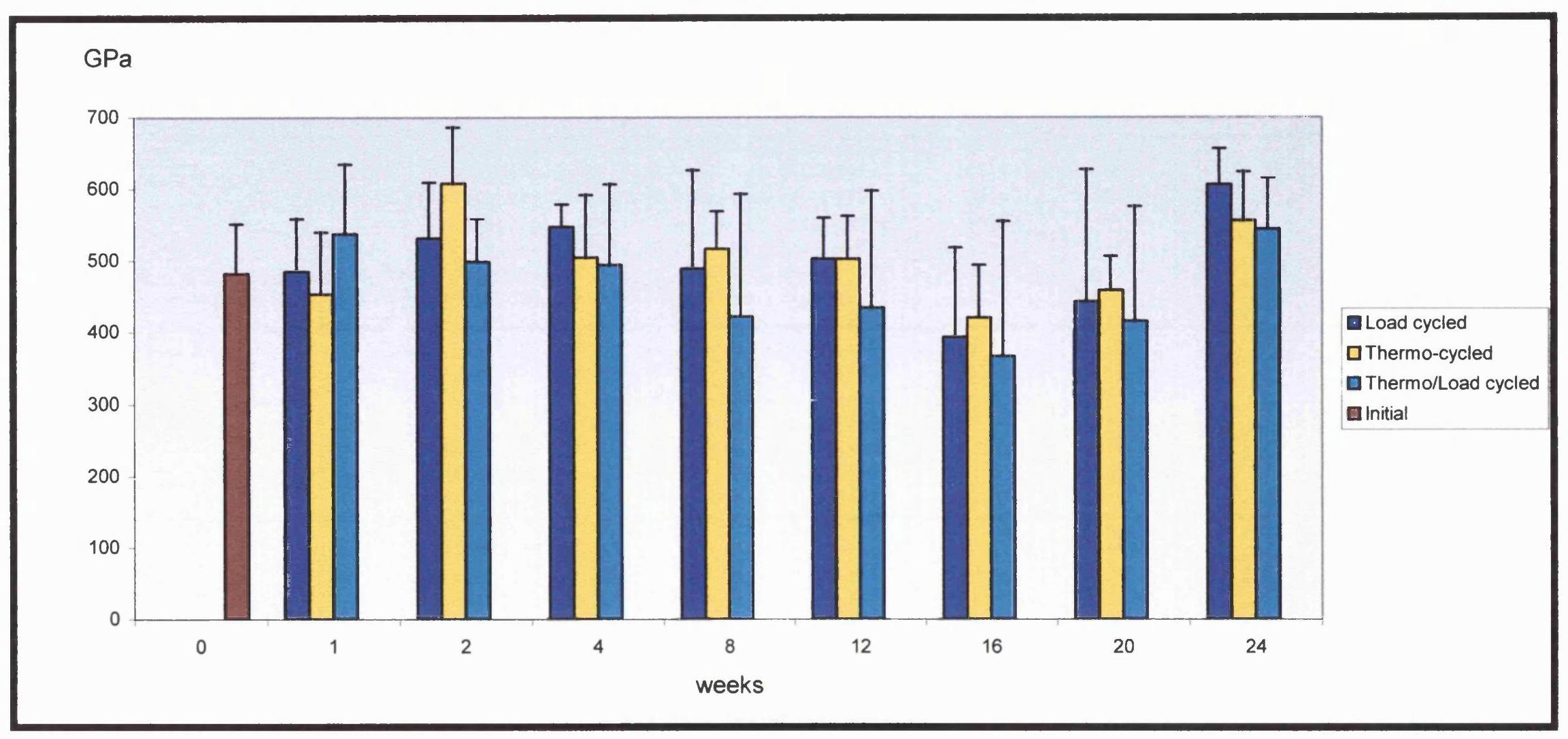

Figure 5.22 : Apparent Flexural Modulus for the different Z100 testing groups (GPa) (Mean values) 


\subsubsection{STRESS AT FAILURE}

This was defined at the point at which the resin spine failed. That point could be detected by the computer program controlling the universal testing machine. When the load decreased abruptly at any point during testing, the computer program regarded it as failure, fracture having already occurred.

\subsubsection{SILUX PLUS}

\subsection{LOAD CYCLED GROUP}

The mean stress at failure value for the bonded Silux Plus resin to metal specimens tested immediately after preparation was $1048.7 \mathrm{MPa}( \pm 238.1)$. The stress at failure results for the Silux Plus specimens subjected to load cycling alone is shown in Table 5.26 and Figure 5.23. Intergroup comparisons were carried out using the Mann-Whitney test and the statistically significant differences between groups are recorded in Table 5.27. During the first week, as the specimens were load cycled, the value obtained for the stress at failure fell significantly to $74 \%$ of its initial value recorded. Subsequently, the values remained relatively unchanged although there was a slight increase in values up to week 4 , followed by a decline up to week 12 when the lowest value measured was recorded. Between week 8 and week 12 there was a $15 \%$ reduction in the value of stress. This is very similar in percentage terms to the reduction of the apparent flexural modulus during the same week period for the load cycled Silux Plus specimens. At week 12 the value had fallen to $72 \%$ of the initial value recorded. Thereafter, there was a trend for the values to increase up to week 24 when the final results were $83 \%$ of the initial recorded value. 


\begin{tabular}{|l|c|c|c|c|c|c|c|c|c|}
\hline Time (Weeks) & $\mathbf{0}$ & $\mathbf{1}$ & $\mathbf{2}$ & $\mathbf{4}$ & $\mathbf{8}$ & $\mathbf{1 2}$ & $\mathbf{1 6}$ & $\mathbf{2 0}$ & $\mathbf{2 4}$ \\
\hline Mean (MPa) & 1048.7 & 780 & 876.2 & 954.3 & 890.7 & 762.9 & 841.6 & 931.3 & 879.1 \\
S/d & 238.1 & 227.4 & 209.1 & 175.2 & 148.4 & 220.1 & 221.5 & 215.6 & 97.5 \\
Median (MPa) & 1038 & 810.8 & 883.9 & 936 & 926.5 & 761.3 & 836.9 & 951.6 & 873.9 \\
\hline
\end{tabular}

Table 5.26 : Stress at failure of Load Cycled Silux Plus (MPa)

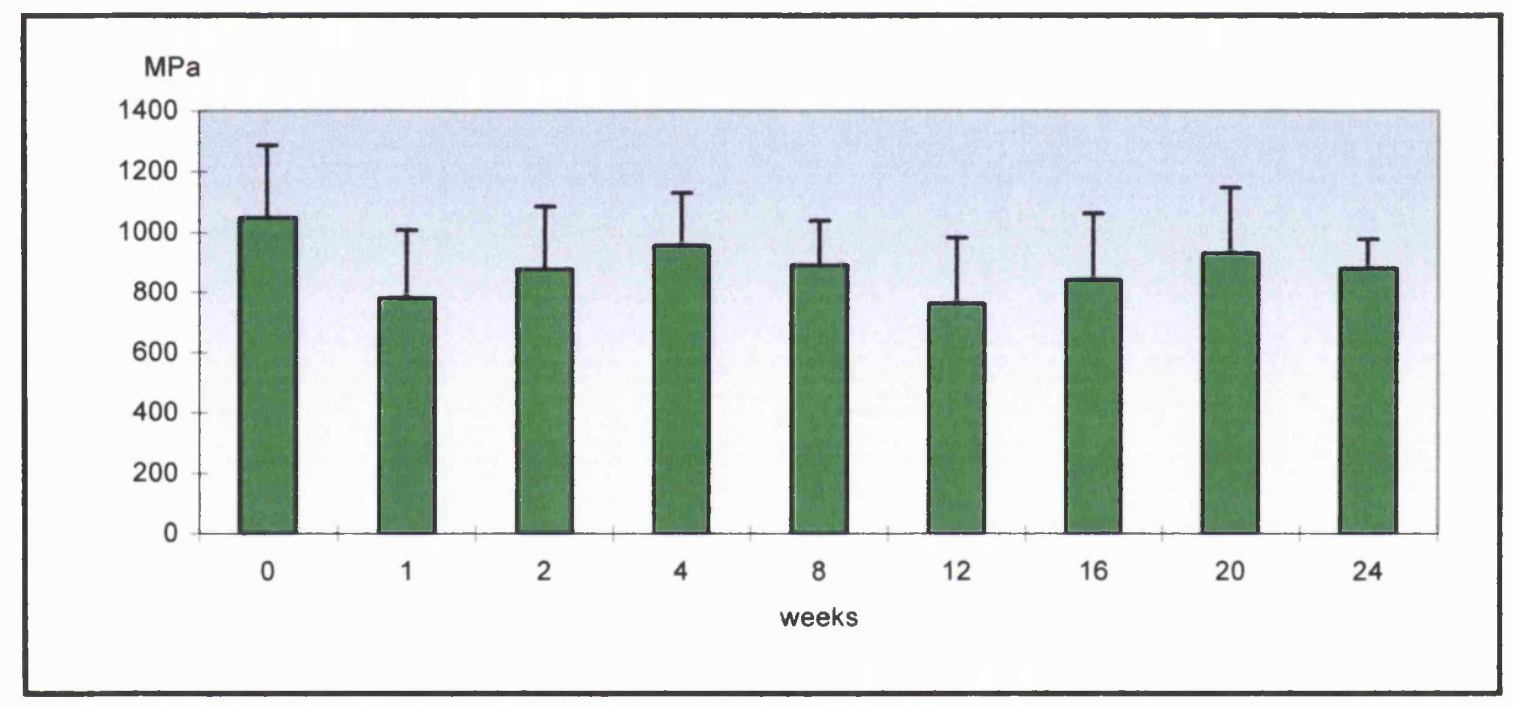

Figure 5.23 : Stress at failure of Load Cycled Silux Plus (MPa)

(Mean values)

\begin{tabular}{|l|l|l|}
\hline Week & 0 & 12 \\
\hline 1 & $S$ & \\
\hline 4 & & $S$ \\
\hline 12 & $S$ & \\
\hline 24 & $S$ & \\
\hline
\end{tabular}

$$
\begin{array}{r}
\mathrm{H} / \mathrm{S}<0.01 \\
\mathrm{~S}<0.05
\end{array}
$$

Table 5.27 : Statistical Analysis of Stress at failure for Load Cycled Silux Plus 


\subsection{THERMO-CYCLED GROUP}

The stress at failure for the Silux Plus specimens subjected to thermo-cycling alone is shown in Table 5.28 and Figure 5.24. Intergroup comparisons were carried out using the Mann-Whitney test and the statistically significant differences between groups are recorded in Table 5.29. A similar pattern was observed to that noted for the load cycled specimens. There was a significant decrease in the mean value of stress at failure over the first week, reaching $57 \%$ of the initial value recorded. Thereafter, a significant increase in the values was observed up to week 8 . At that point the values recorded were $91 \%$ of their initial value. This same trend was observed with the load cycled group. A $20 \%$ drop in the value was noted between weeks 8 and 12. Again, the fall is very similar to the reduction of the apparent flexural modulus over the same period for the thermo-cycled Silux Plus specimens. After week 12, the value begun to change for the thermo-cycled specimens, showing an increase, which reached $84 \%$ of the initial value recorded by the end of the experiment. 


\begin{tabular}{|l|c|c|c|c|c|c|c|c|c|}
\hline Time (Weeks) & $\mathbf{0}$ & $\mathbf{1}$ & $\mathbf{2}$ & $\mathbf{4}$ & $\mathbf{8}$ & $\mathbf{1 2}$ & $\mathbf{1 6}$ & $\mathbf{2 0}$ & $\mathbf{2 4}$ \\
\hline Mean (MPa) & 1048.7 & 601.7 & 771.4 & 918.5 & 957.2 & 781.4 & 845.1 & 994.1 & 881.3 \\
S/d & 238.1 & 229.5 & 128.0 & 121.3 & 362.8 & 287.1 & 90.1 & 344.8 & 110.8 \\
Median (MPa) & 1038 & 533.6 & 763.3 & 897 & 934.4 & 738 & 807.3 & 886.4 & 887.2 \\
\hline
\end{tabular}

Table 5.28 : Stress at failure of Thermo-Cycled Silux Plus (MPa)

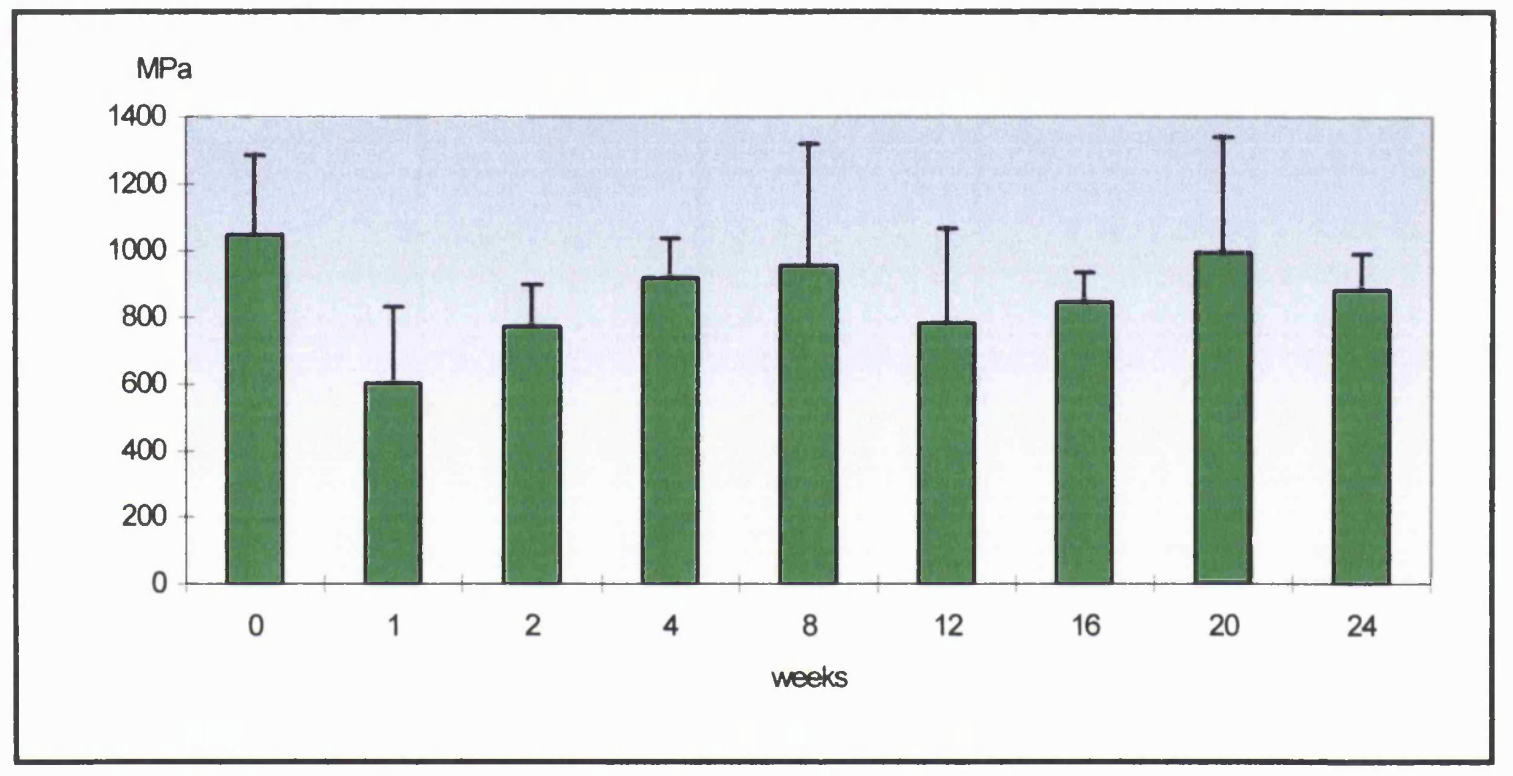

Figure 5.24 : Stress at failure of Thermo-Cycled Silux Plus (MPa) (Mean values)

\begin{tabular}{|l|c|c|c|c|c|}
\hline Week & 0 & 4 & 8 & 16 & 24 \\
\hline 1 & H/S & H/S & S & S & H/S \\
\hline 2 & H/S & S & & & \\
\hline 12 & S & & & & \\
\hline 16 & S & & & & \\
\hline 24 & S & & & & \\
\hline
\end{tabular}

$\mathrm{H} / \mathrm{S}<0.01$
$\mathrm{~S}<0.05$

Table 5.29 : Statistical Analysis of Stress at failure for Thermo-Cycled Silux Plus 


\subsection{THERMO/LOAD CYCLED GROUP}

The stress at failure for the Silux Plus specimens subjected to both thermo and load cycling is shown in Table 5.30 and Figure 5.25. Intergroup comparisons were carried out using the Mann-Whitney test and the statistically significant differences between groups are recorded in Table 5.31. The same pattern was observed with the specimens subjected to both thermo and load cycling as in the specimens when either thermo or load cycled was carried out alone. There was a significant reduction in the stress at failure compared to the one at the start of the experiment. The values obtained were only $55 \%$ of the initial value. The increase in the values found at subsequent time intervals was greater in the group subjected to both thermo and load cycling, when compared to the groups thermo or load cycled alone.

Values of the stress at failure for the thermo/load cycled group obtained at the end of week 2 , showed a $42 \%$ increase compared to week 1 . The variations observed previously in the groups that they were either thermo or load cycled alone were also noted with this group up to week 12 . Further, the variation in the values was very similar to the changes in the apparent flexural modulus over the same period for the combined thermo and load cycled Silux Plus specimens. By the end of the test period, the values were $78 \%$ of the initial values recorded. 


\begin{tabular}{|l|c|c|c|c|c|c|c|c|c|}
\hline Time (Weeks) & $\mathbf{0}$ & $\mathbf{1}$ & $\mathbf{2}$ & $\mathbf{4}$ & $\mathbf{8}$ & $\mathbf{1 2}$ & $\mathbf{1 6}$ & $\mathbf{2 0}$ & $\mathbf{2 4}$ \\
\hline Mean (MPa) & 1048.7 & 586.8 & 1011 & 778.8 & 894.8 & 805.2 & 824.1 & 896.3 & 828.4 \\
S/d & 238.1 & 343.2 & 308.8 & 145.2 & 179.8 & 139.4 & 89.5 & 139.7 & 122.1 \\
Median (MPa) & 1038 & 510.2 & 945.4 & 825.7 & 892.5 & 821.6 & 800.3 & 903.1 & 827.2 \\
\hline
\end{tabular}

Table 5.30 : Stress at failure of Thermo/Load Cycled Silux Plus (MPa)

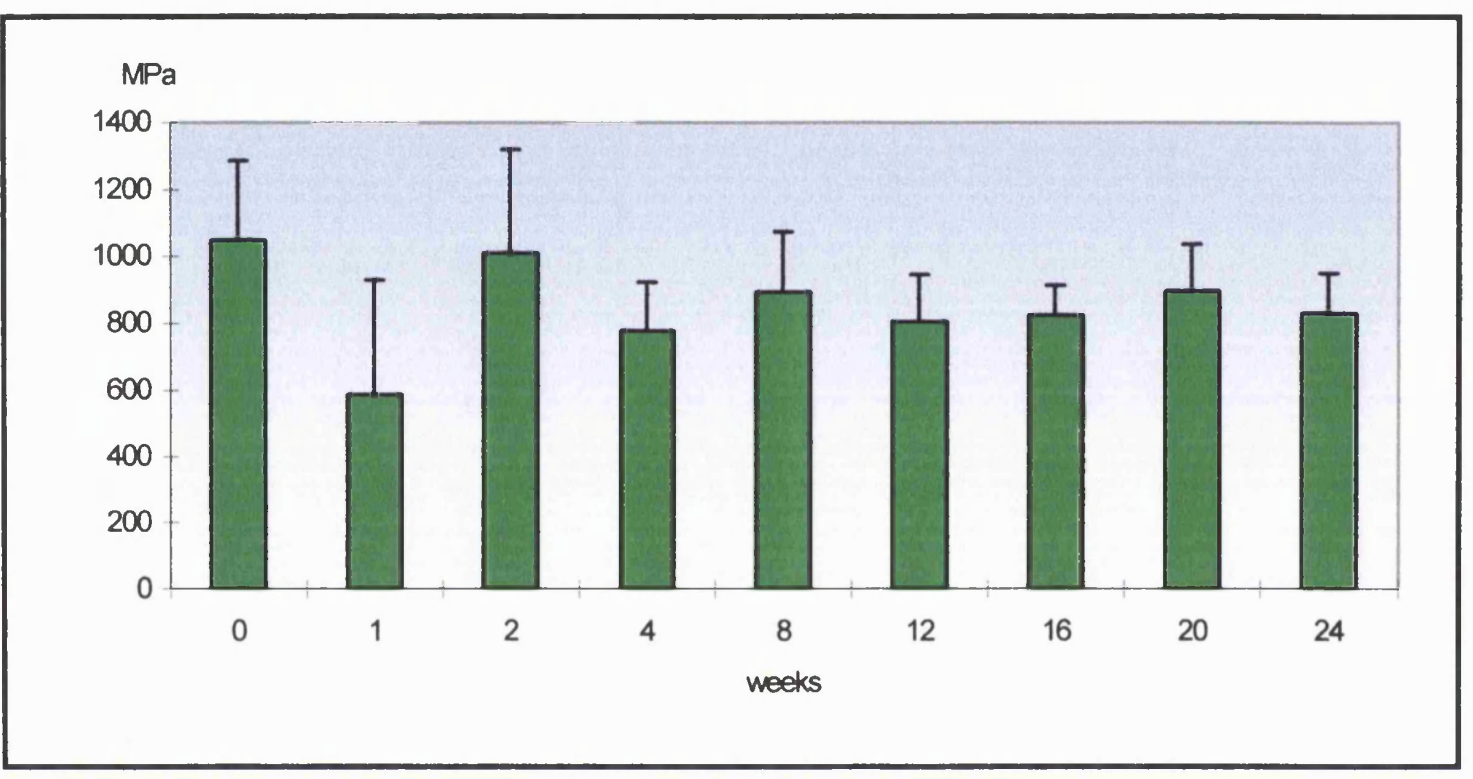

Figure 5.25 : Stress at failure of Thermo/Load Cycled Silux Plus (MPa) (Mean values)

\begin{tabular}{|l|c|c|c|c|c|c|c|c|}
\hline Week & 0 & 2 & 4 & 8 & 12 & 16 & 20 & 24 \\
\hline 1 & S & S & S & S & S & S & S & S \\
\hline 4 & H/S & & & & & & & \\
\hline 12 & S & & & & & & & \\
\hline 16 & H/S & & & & & & & \\
\hline 20 & S & & & & & & & \\
\hline 24 & S & & & & & & & \\
\hline
\end{tabular}

$\mathrm{H} / \mathrm{S}<0.01$

$\mathrm{S}<0.05$

Table 5.31 : Statistical Analysis of Stress at failure for Thermo/Load Cycled Silux Plus 


\subsection{COMPARISON OF THE DIFFERENT SILUX PLUS CYCLING}

\section{GROUPS}

Inter group comparison of the stress at failure results for Silux Plus is shown in Figure 5.26. Intergroup comparisons were carried out between treatments at similar time intervals using the Mann-Whitney test and the statistically significant differences between groups are recorded in Table 5.32. There was a statistical significant difference in between the week 4 load cycled group and the week 4 combined thermo and load cycled group. The lowest value showed was for the combined thermo and load cycled group.

\begin{tabular}{|c|c|c|}
\hline Week & & 4 \\
\hline & Group & $\begin{array}{c}\text { Thermo/Load } \\
\text { cycled }\end{array}$ \\
\hline 4 & $\begin{array}{c}\text { Load } \\
\text { cycled }\end{array}$ & $\mathrm{S}$ \\
\hline
\end{tabular}

$\mathrm{H} / \mathrm{S}<0.01$

$\mathrm{S}<0.05$

Table 5.32 : Statistical Analysis of Stress at failure for the different Silux Plus testing groups 


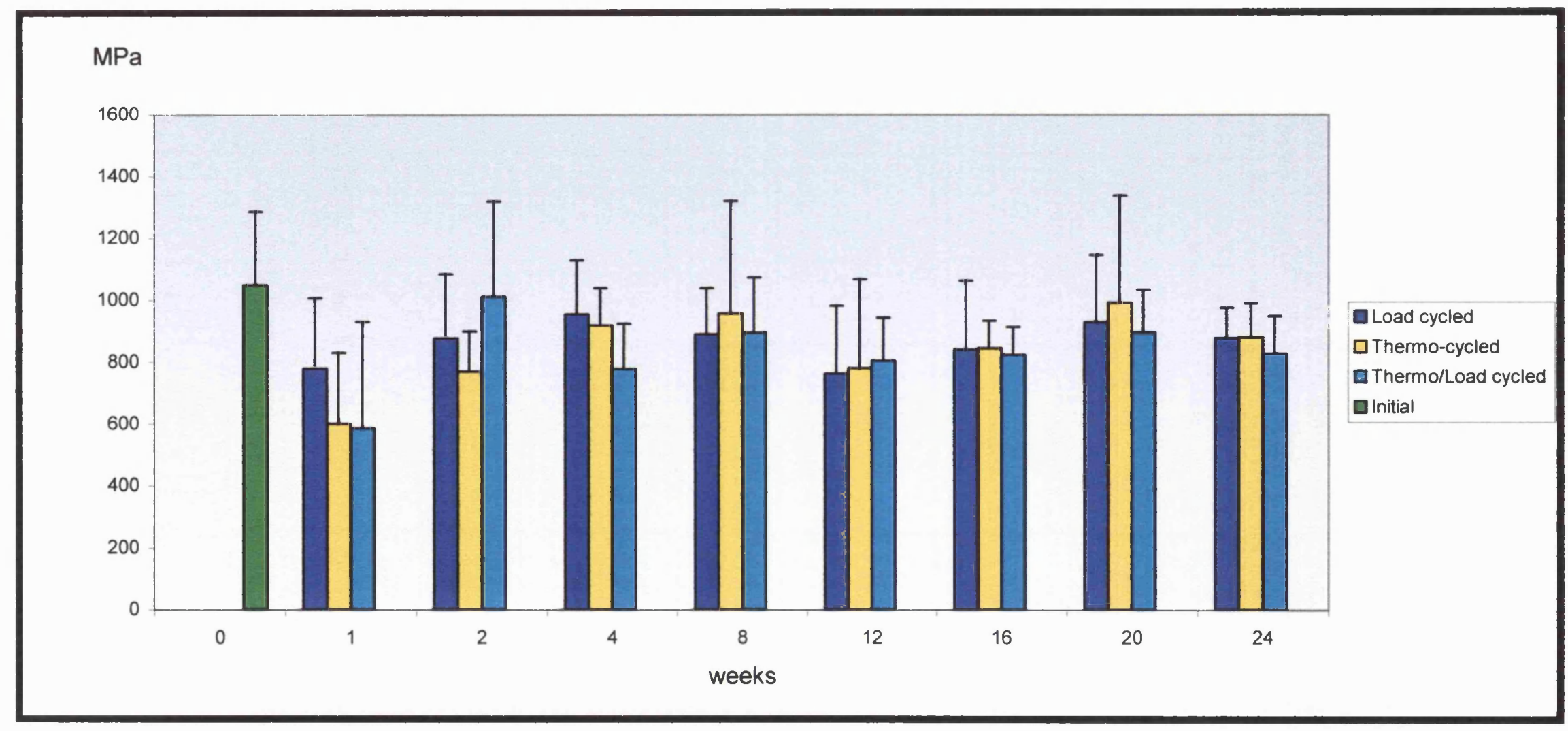

Figure 5.26 : Stress at failure for the different Silux Plus testing groups (MPa) (Mean values) 


\subsubsection{Z100}

\subsection{LOAD CYCLED GROUP}

The mean stress at failure for the bonded Z100 resin to metal specimens tested immediately after preparation was $1042 \mathrm{MPa}( \pm 151.7)$. The results for the Z100 specimens subjected to load cycling only are shown in Table 5.33 and Figure 5.27. Intergroup comparisons were carried out using the Mann-Whitney test and the statistically significant differences between groups are recorded in Table 5.34.

After the specimens were load cycled for one week, there was a $23 \%$ reduction in the value of the stress at failure. This percentage reduction was very similar to the reduction seen on the Silux Plus load cycled specimens.

At subsequent test intervals, the value of the stress at failure remained relatively unchanged although small cyclical changes were noted. At week 16 the value was the lowest observed being only $56 \%$ the initial value. This pattern was similar to that observed for Silux Plus however for Silux Plus the fall occurred over a shorter period of time (week 12). At week 16 the value for the stress at failure was $70 \%$ the value at 12 weeks. This reduction is very similar to that observed for the apparent flexural modulus during the same period for the same material under the same conditions.

During the final 8 weeks of the experiment the mean stress at failure value increased by $36 \%$. This is very similar in terms of percentage to the increase of the apparent flexural modulus for the $\mathrm{Z} 100$ load cycled specimens during the same week period. 


\begin{tabular}{|l|c|c|c|c|c|c|c|c|c|}
\hline Time (Weeks) & $\mathbf{0}$ & $\mathbf{1}$ & $\mathbf{2}$ & $\mathbf{4}$ & $\mathbf{8}$ & $\mathbf{1 2}$ & $\mathbf{1 6}$ & $\mathbf{2 0}$ & $\mathbf{2 4}$ \\
\hline Mean (MPa) & 1042 & 804.4 & 956.1 & 1043.3 & 912.8 & 832 & 590.1 & 620.4 & 919.9 \\
S/d & 151.7 & 233.4 & 308.8 & 363.2 & 355.0 & 221.9 & 173.3 & 227.2 & 108.6 \\
Median (MPa) & 1060 & 780.8 & 1032 & 1074 & 957.5 & 912.5 & 580.2 & 587.2 & 909 \\
\hline
\end{tabular}

Table 5.33 : Stress at failure of Load Cycled Z100 (MPa)

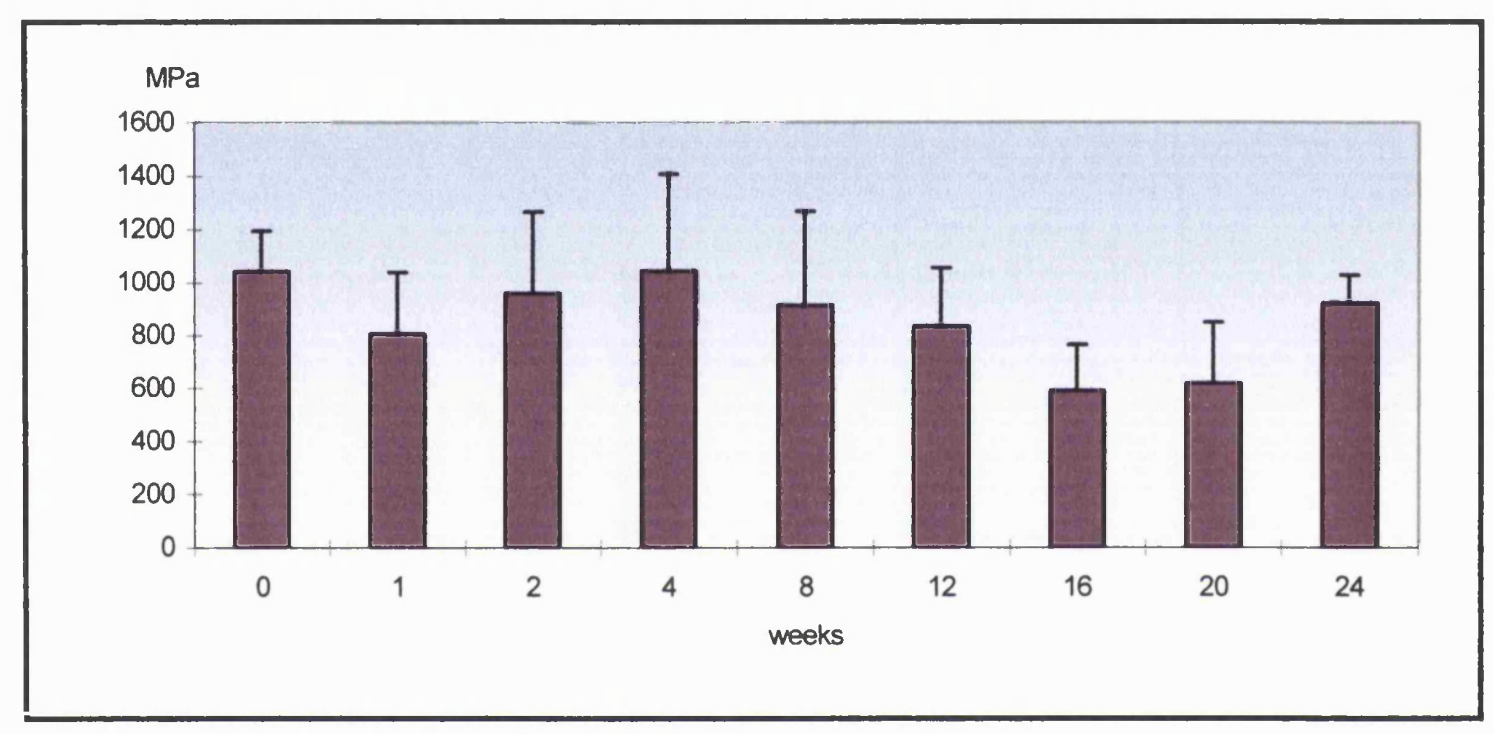

Figure 5.27 : Stress at failure of Load Cycled Z100 (MPa) (Mean values)

\begin{tabular}{|l|c|c|c|c|}
\hline Week & O & 16 & 20 & 24 \\
\hline 1 & S & S & & \\
\hline 2 & & S & & \\
\hline 4 & & H/S & & \\
\hline 12 & S & S & & \\
\hline 16 & H/S & & & H/S \\
\hline 20 & H/S & & & \\
\hline 24 & & & S & \\
\hline
\end{tabular}

$\mathrm{H} / \mathrm{S}<0.01$

$\mathrm{S}<0.05$

Table 5.34 : Statistical Analysis of Stress at failure for Load Cycled Z100 


\subsection{THERMO-CYCLED GROUP}

The stress at failure for the Z100 specimens subjected to thermo-cycling only is shown in Table 5.35 and Figure 5.28. Intergroup comparisons were carried out using the MannWhitney test and the statistically significant differences between groups are recorded in Table 5.36. The thermo-cycled specimens behaved in a similar way to the load cycled group. However, the fall in values occurred after week 2.

Between week 2 and week 4 a $17 \%$ reduction in the value can be seen. This reduction was less, compared to the load cycled group, but very similar to the reduction in the apparent flexural modulus during the same period for the same material under the same conditions. It was observed that the reduction in the stress value occurred sooner with the thermocycled specimens than with the load cycled ones.

From week 16 up to week 24 the value increased $25 \%$. This percentage was similar to the percentage increase of the apparent flexural modulus during the same period for the same material under the same conditions. However, the increase in the stress value for the thermo-cycled group was less than the increase in the stress value for the load cycled specimens between weeks 16 and 24 . 


\begin{tabular}{|l|c|c|c|c|c|c|c|c|c|}
\hline Time (Weeks) & $\mathbf{0}$ & $\mathbf{1}$ & $\mathbf{2}$ & $\mathbf{4}$ & $\mathbf{8}$ & $\mathbf{1 2}$ & $\mathbf{1 6}$ & $\mathbf{2 0}$ & $\mathbf{2 4}$ \\
\hline Mean (MPa) & 1042 & 949.1 & 1094 & 867.8 & 989.5 & 927.5 & 820.8 & 990.1 & 840 \\
S/d & 151.7 & 232.4 & 318.3 & 281.9 & 219.5 & 292.9 & 248.7 & 220.4 & 185.0 \\
Median (MPa) & 1060 & 979.2 & 1046 & 885.4 & 1032 & 989.5 & 797.1 & 1027 & 861 \\
\hline
\end{tabular}

Table 5.35 : Stress at failure of Thermo-Cycled Z100 (MPa)

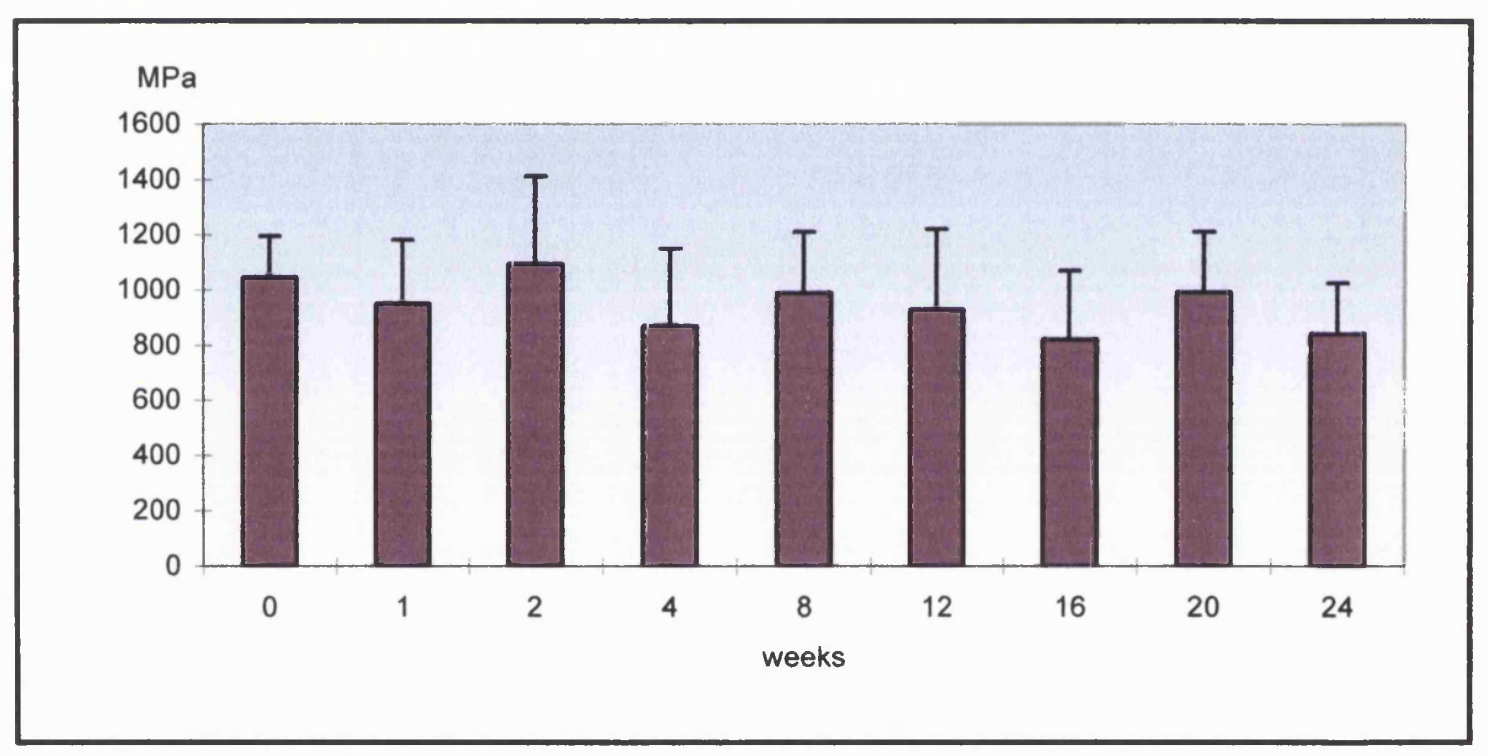

Figure 5.28 : Stress at failure of Thermo-Cycled Z100 (MPa)

(Mean values)

\begin{tabular}{|l|c|c|c|}
\hline Week & 0 & 16 & 24 \\
\hline 2 & & S & S \\
\hline 16 & S & & \\
\hline 24 & S & & \\
\hline
\end{tabular}

$\mathrm{H} / \mathrm{S}<0.01$

$\mathrm{S}<0.05$

Table 5.36 : Statistical Analysis of Stress at failure for Thermo-Cycled Z100 


\subsection{THERMO/LOAD CYCLED GROUP}

The stress at failure for the $\mathrm{Z} 100$ specimens subjected to both thermo and load cycling is shown in Table 5.37 and Figure 5.29. Intergroup comparisons were carried out using the Mann-Whitney test and the statistically significant differences between groups are recorded in Table 5.38. The mean stress at failure for the specimens that were subjected to both thermo and load cycling remained relatively unchanged up to week 2 . There was a $44 \%$ reduction in the stress at failure in between week 2 and week 4 . This reduction was higher in terms of percentage to that observed for the thermo-cycled group.

The stress values remained relatively unchanged up to week 24 when the value was $79 \%$ of the initial value recorded in the start of the experiment. 


\begin{tabular}{|l|c|c|c|c|c|c|c|c|c|}
\hline Time (Weeks) & $\mathbf{0}$ & $\mathbf{1}$ & $\mathbf{2}$ & $\mathbf{4}$ & $\mathbf{8}$ & $\mathbf{1 2}$ & $\mathbf{1 6}$ & $\mathbf{2 0}$ & $\mathbf{2 4}$ \\
\hline Mean (MPa) & 1042 & 941.3 & 1136.4 & 638.3 & 812.8 & 894.2 & 720 & 663 & 833.3 \\
S/d & 151.7 & 270.6 & 259.6 & 268.1 & 368.5 & 420.2 & 411 & 223.5 & 355.8 \\
Median (MPa) & 1060 & 919.4 & 1168.5 & 601 & 942.2 & 961.3 & 615.2 & 608.9 & 803.6 \\
\hline
\end{tabular}

Table 5.37 : Stress at failure of Thermo/Load Cycled Z100 (MPa)

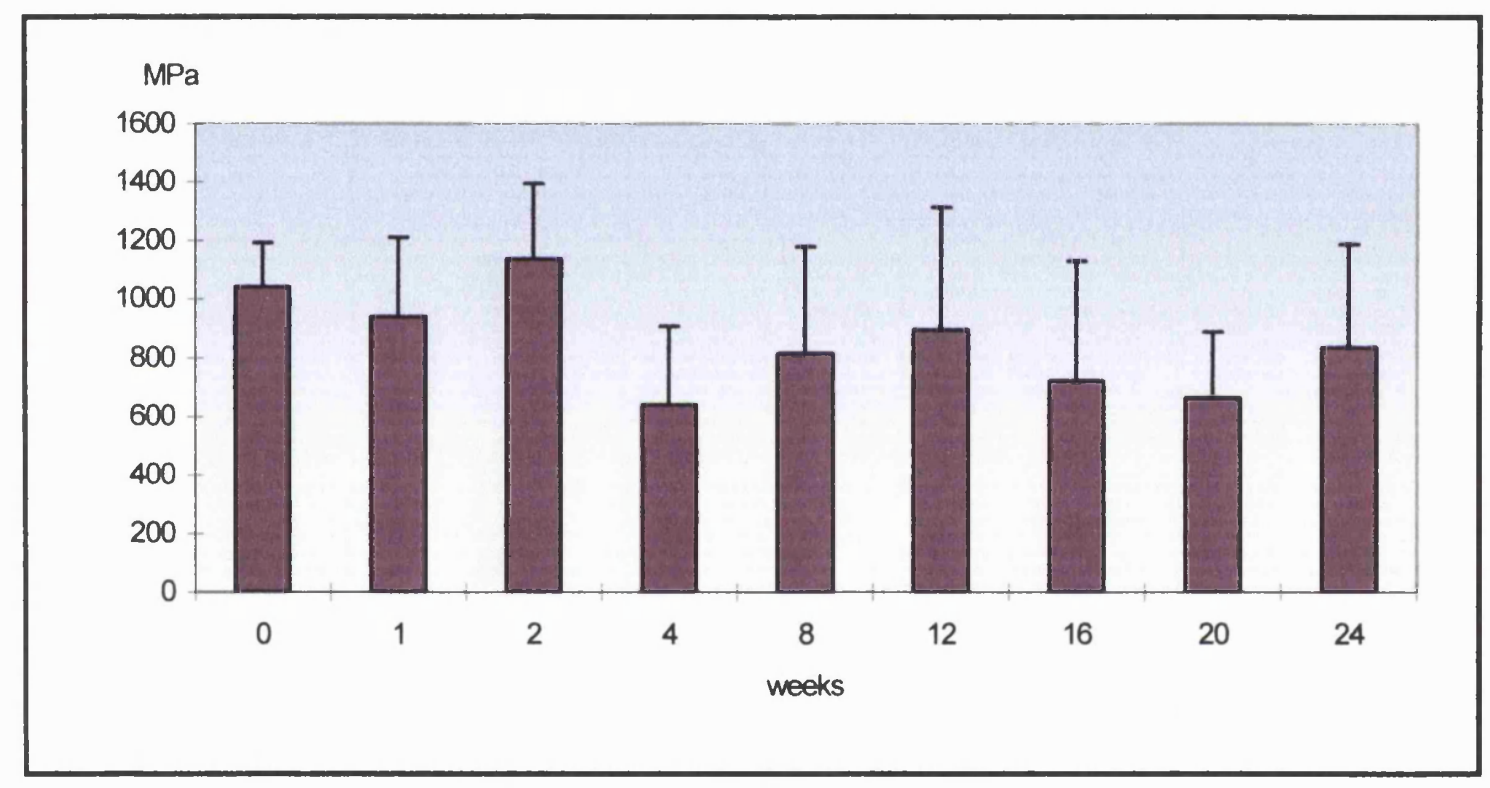

Figure 5.29 : Stress at failure of Thermo/Load Cycled Z100 (MPa) (Mean values)

\begin{tabular}{|l|c|c|c|c|c|}
\hline Week & 0 & 4 & 8 & 16 & 20 \\
\hline 1 & & S & & & \\
\hline 2 & & H/S & S & S & S \\
\hline 4 & H/S & & & & \\
\hline 20 & S & & & & \\
\hline
\end{tabular}

$\mathrm{H} / \mathrm{S}<0.01$

$\mathrm{S}<0.05$

Table 5.38 : Statistical Analysis of Stress at failure for Thermo/Load Cycled Z100 


\subsection{COMPARISON OF THE DIFFERENT Z100 CYCLING GROUPS}

Inter group comparison of the stress at failure results for $\mathrm{Z100}$ is shown in Figure 5.30. Intergroup comparisons were carried out between treatments at similar time intervals using the Mann-Whitney test and the statistically significant differences between groups are recorded in Table 5.39. There were significant differences between week 4 load cycled group and combined thermo/load cycled group, the latter group having the lower value. There were significant differences between the week 16 load cycled group and thermo-cycled group. There was also highly significant differences in between week 20 load cycled group and thermo-cycled group. The load cycled group on both occasions exhibited a lower peak value.

\begin{tabular}{|c|c|c|c|c|}
\hline Week & & 4 & 16 & 20 \\
\hline & Group & $\begin{array}{c}\text { Thermo/Load } \\
\text { cycled }\end{array}$ & $\begin{array}{l}\text { Thermo- } \\
\text { cycled }\end{array}$ & $\begin{array}{l}\text { Thermo- } \\
\text { cycled }\end{array}$ \\
\hline 4 & $\begin{array}{l}\text { Load } \\
\text { cycled }\end{array}$ & $\mathbf{S}$ & & \\
\hline 16 & $\begin{array}{l}\text { Load } \\
\text { cycled }\end{array}$ & & $\mathbf{S}$ & \\
\hline 20 & $\begin{array}{l}\text { Load } \\
\text { cycled }\end{array}$ & & & $\mathbf{H} / \mathbf{S}$ \\
\hline
\end{tabular}

$\mathrm{H} / \mathrm{S}<0.01$

$\mathrm{S}<0.05$

Table 5.39 : Statistical Analysis of Stress at failure for the different Z100 testing groups 


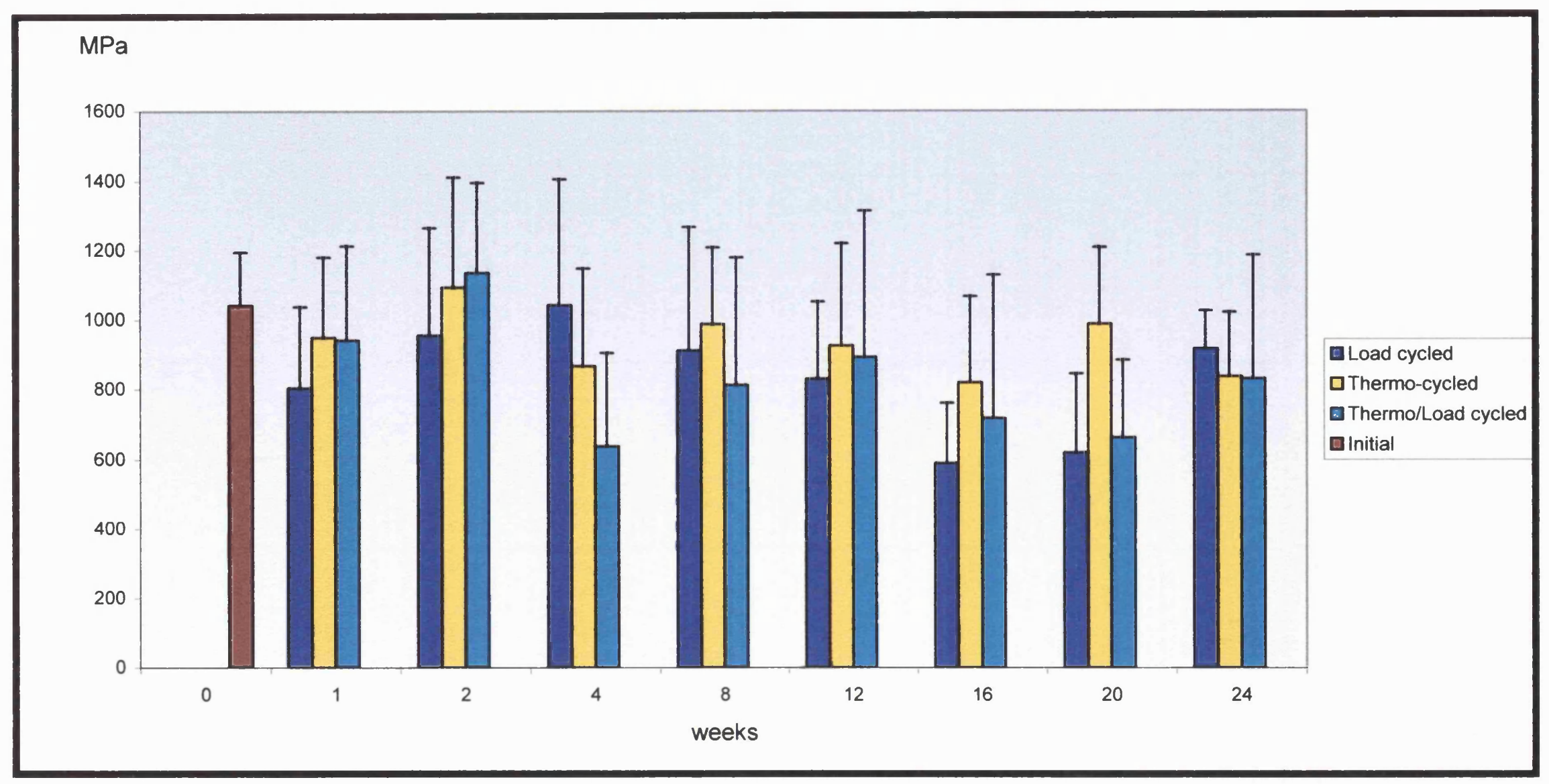

Figure 5.30 : Stress at failure for the different Z100 testing groups (MPa) (Mean values) 


\subsubsection{EXTENSION AT FAILURE}

When the specimens were tested to fracture, the extension at failure was also noted. The mean extension at failure of all specimens (Load cycled + Thermo-cycled + Load/Thermo-cycled) for each material at every week period was calculated. Figure 5.31 summarises the comparative mean values between materials, of all specimens at every week period. As it can be appreciated from Figure 5.31, Z100 tends to show a lower extension at failure compared to Silux Plus throughout testing.

\subsubsection{STATISTICAL ANALYSIS TO VERIFY CHANGES WITHIN AND BETWEEN TESTING TIMES}

To verify differences between groups associated with the different durations of testing, Mann-Whitney tests were performed between the total population of specimens (i.e. Load cycled + Thermo-cycled + Load/thermo-cycled) of each week. The statistical results for Silux Plus are shown in Table 5.40 (apparent flexural modulus) and 5.41(stress at failure). ? they

For Z100 are shown in Table 5.42 (apparent flexural modulus) and 5.43 (stress at failure). When possible changes in the behaviour of a particular test group in relation to other specimens of the same week were investigated, no significant differences were observed apart from the Z100 week 20 thermo-cycled group compared to the total population of the $\mathrm{Z} 100$ at week 20 . Here a significant difference was observed. 


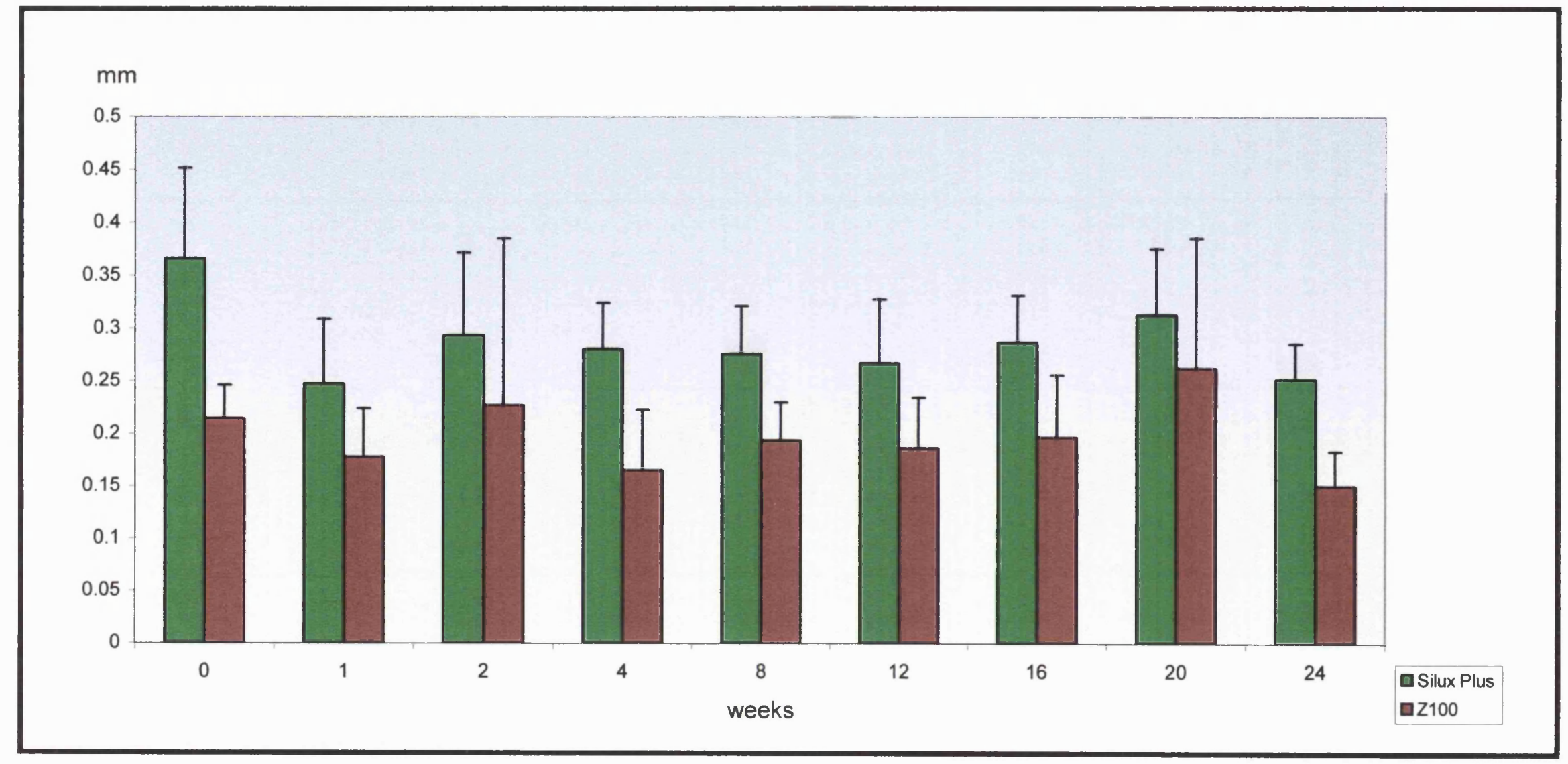

Figure 5.31 : Extension at failure for the different testing groups $(\mathrm{mm})$ (Mean values) 


\begin{tabular}{|l|c|c|c|c|c|c|c|}
\hline Week & 1 & 2 & 4 & 8 & 12 & 16 & 20 \\
\hline 4 & $\mathrm{H} / \mathrm{S}$ & & & & $\mathrm{S}$ & $\mathrm{S}$ & $\mathrm{S}$ \\
\hline 8 & $\mathrm{~S}$ & $\mathrm{~S}$ & & & $\mathrm{~S}$ & $\mathrm{H} / \mathrm{S}$ & \\
\hline 20 & & & & $\mathrm{H} / \mathrm{S}$ & & & \\
\hline 24 & $\mathrm{H} / \mathrm{S}$ & $\mathrm{H} / \mathrm{S}$ & $\mathrm{H} / \mathrm{S}$ & & $\mathrm{H} / \mathrm{S}$ & $\mathrm{H} / \mathrm{S}$ & $\mathrm{H} / \mathrm{S}$ \\
\hline
\end{tabular}

$\mathrm{H} / \mathrm{S}<0.01$

$\mathrm{S}<0.05$

Table 5.40 : Statistical analysis of Apparent Flexural Modulus of each week Silux Plus population compared to the rest of populations of other weeks

\begin{tabular}{|l|c|c|c|c|c|c|}
\hline Week & 1 & 2 & 4 & 8 & 12 & 24 \\
\hline 2 & $\mathrm{~S}$ & & $\mathrm{~S}$ & & $\mathrm{~S}$ & \\
\hline 16 & $\mathrm{H} / \mathrm{S}$ & $\mathrm{H} / \mathrm{S}$ & $\mathrm{S}$ & $\mathrm{H} / \mathrm{S}$ & $\mathrm{H} / \mathrm{S}$ & $\mathrm{H} / \mathrm{S}$ \\
\hline 20 & $\mathrm{~S}$ & $\mathrm{H} / \mathrm{S}$ & & $\mathrm{S}$ & & \\
\hline \multicolumn{6}{c|}{$\begin{array}{c}\mathrm{H} / \mathrm{S}<0.01 \\
\mathrm{~S}<0.05\end{array}$} \\
\hline
\end{tabular}

Table 5.41 : Statistical analysis of Stress at Failure of each week Silux Plus population compared to the rest of populations of other weeks 


\begin{tabular}{|l|c|c|c|c|c|c|c|}
\hline Week & 1 & 2 & 4 & 8 & 12 & 16 & 20 \\
\hline 2 & $\mathrm{~S}$ & & & & $\mathrm{~S}$ & & \\
\hline 16 & $\mathrm{H} / \mathrm{S}$ & $\mathrm{H} / \mathrm{S}$ & $\mathrm{H} / \mathrm{S}$ & $\mathrm{H} / \mathrm{S}$ & $\mathrm{H} / \mathrm{S}$ & & \\
\hline 20 & $\mathrm{H} / \mathrm{S}$ & $\mathrm{H} / \mathrm{S}$ & $\mathrm{H} / \mathrm{S}$ & $\mathrm{H} / \mathrm{S}$ & & & \\
\hline 24 & $\mathrm{H} / \mathrm{S}$ & & $\mathrm{S}$ & $\mathrm{H} / \mathrm{S}$ & $\mathrm{H} / \mathrm{S}$ & $\mathrm{H} / \mathrm{S}$ & $\mathrm{H} / \mathrm{S}$ \\
\hline
\end{tabular}

$\mathrm{H} / \mathrm{S}<0.01$

$\mathrm{S}<0.05$

Table 5.42 : Statistical analysis of Apparent Flexural Modulus of each week Z100 population compared to the rest of populations of other weeks

\begin{tabular}{|c|c|c|c|c|c|c|}
\hline Week & 1 & 2 & 4 & 8 & 12 & 24 \\
\hline 2 & $\mathrm{~S}$ & & $\mathrm{~S}$ & & $\mathrm{~S}$ & \\
\hline 16 & $\mathrm{H} / \mathrm{S}$ & $\mathrm{H} / \mathrm{S}$ & $\mathrm{S}$ & $\mathrm{H} / \mathrm{S}$ & $\mathrm{H} / \mathrm{S}$ & $\mathrm{H} / \mathrm{S}$ \\
\hline 20 & $\mathrm{~S}$ & $\mathrm{H} / \mathrm{S}$ & & $\mathrm{S}$ & & \\
\hline
\end{tabular}

$\mathrm{H} / \mathrm{S}<0.01$

$\mathrm{S}<0.05$

Table 5.43 : Statistical analysis of Stress at Failure of each week Z100 population compared to the rest of populations of other weeks 


\subsubsection{MODE OF FAILURE AND DYE PENETRATION TEST}

All specimens of the 1 week, 2 weeks, 16 weeks, 20 weeks and 24 weeks groups were selected to undergo a dye penetration test and to determine their mode of failure. This permitted comparison of the results for the conditioning process. During the dye penetration study, it was noted that in some cohesive failures, the fracture line went in both directions, on either side of the original fracture line approaching the metal surface. This is shown in Figures $5.32 \mathrm{a}+\mathrm{b}$. Cohesive failure where the fracture line was not divided is shown in Figures $5.33 \mathrm{a}+\mathrm{b}$. A typical adhesive failure is shown in Figures $5.34 \mathrm{a}+\mathrm{b}$. The results of the dye penetration measurements for Silux Plus are shown in Table 5.44 and for $\mathrm{Z} 100$ in Table 5.45. In the cases of cohesive failure that the fracture line was divided the distances on either side of the central crack were recorded. The mode of failure for Silux Plus is also shown in Table 5.46 and for Z100 in Table 5.47. 


\begin{tabular}{|c|c|c|c|c|}
\hline 1 week & 2 weeks & 16 weeks & 20 weeks & 24 weeks \\
\hline Thermo-cycled & Thermo-cycled & Thermo-cycled & Thermo-cycled & Thermo-cycled \\
\hline \begin{tabular}{|c|}
7.5 \\
8.6 \\
9.7 \\
10.2 \\
10.8 \\
11.1 \\
11.4 \\
12.7 \\
13.4 \\
15.2
\end{tabular} & $\begin{array}{c}3.4 \\
4.4 \\
4.5 \\
4.5 \\
4.5 \\
6.2 \\
1.4+3.5 \\
2.0+3.4 \\
3.0+5.5 \\
6.0+3.2\end{array}$ & $\begin{array}{c}4.5 \\
4.7 \\
5.2 \\
7.3 \\
7.4 \\
8.2 \\
9.3 \\
4.1+3.7 \\
4.1+7.3 \\
4.3+3.9\end{array}$ & $\begin{array}{c}2.0 \\
2.7 \\
3.6 \\
5.0 \\
5.3 \\
5.8 \\
6.1 \\
2.3+3.0 \\
3.2+1.1 \\
3.3+2.1\end{array}$ & $\begin{array}{c}6.7 \\
13.6 \\
16.3 \\
1.6+1.1 \\
8.7 \\
9.2 \\
11.2 \\
11.4 \\
12.7 \\
15.0\end{array}$ \\
\hline Load cycled & Load cycled & Load cycled & Load cycled & Load cycled \\
\hline \begin{tabular}{|c|}
4.3 \\
5.3 \\
7.3 \\
9.3 \\
10.4 \\
12.3 \\
12.4 \\
12.5 \\
16.5 \\
17.0
\end{tabular} & $\begin{array}{c}3.4 \\
3.9 \\
5.9 \\
6.2 \\
6.5 \\
11.4 \\
3.9+4.5 \\
4.3+1.9 \\
4.8+3.9 \\
20.3\end{array}$ & $\begin{array}{c}3.6 \\
4.9 \\
5.0 \\
7.2 \\
2.3+3.3 \\
2.5+1.7 \\
3.4+2.0 \\
4.3+2.6 \\
4.5+2.8 \\
5.3+1.2\end{array}$ & $\begin{array}{c}3.3 \\
4.3 \\
5.2 \\
5.4 \\
2.3+4.5 \\
2.4+1.7 \\
3.2+2.8 \\
4.4+3.7 \\
4.5+3.9 \\
10.6\end{array}$ & $\begin{array}{c}3.7 \\
3.9 \\
6.2 \\
7.3 \\
1.5+2.3 \\
4.3+3.6 \\
6.4+1.6 \\
10.0+4.5 \\
11.0 \\
15.2\end{array}$ \\
\hline $\begin{array}{c}\text { Thermo/Load } \\
\text { cycled }\end{array}$ & $\begin{array}{c}\text { Thermo/Load } \\
\text { cycled }\end{array}$ & $\begin{array}{l}\text { Thermo/Load } \\
\text { cycled }\end{array}$ & $\begin{array}{l}\text { Thermo/Load } \\
\text { cycled }\end{array}$ & $\begin{array}{c}\text { Thermo/Load } \\
\text { Cycled }\end{array}$ \\
\hline \begin{tabular}{|c|}
7.3 \\
7.7 \\
7.8 \\
8.3 \\
8.4 \\
9.4 \\
12.3 \\
17.0 \\
17.0 \\
17.3
\end{tabular} & $\begin{array}{c}2.3 \\
3.5 \\
4.0 \\
5.0 \\
6.4 \\
1+3.3 \\
1.5+3.3 \\
2.3+3.4 \\
3.1+2.1 \\
7.4+3.5\end{array}$ & $\begin{array}{c}2.4 \\
3.0 \\
4.3 \\
5.3 \\
6.2 \\
2.0+3.1 \\
2.8+3.5 \\
3.5+5.0 \\
4.0+3.5 \\
4.4+3.8\end{array}$ & $\begin{array}{c}4.3 \\
4.5 \\
4.6 \\
5.0 \\
5.5 \\
6.0 \\
6.2 \\
2.4+3.2 \\
3.2+1.2 \\
5.3+2.0\end{array}$ & \begin{tabular}{|c|}
1.85 \\
6.3 \\
8.2 \\
8.3 \\
8.4 \\
10.2 \\
11.4 \\
14.4 \\
14.8 \\
16.2
\end{tabular} \\
\hline
\end{tabular}

Cohesive failure: ${ }^{\bullet}$ Adhesive failure: ${ }^{\bullet}$

Table 5.44 : Silux Plus dye penetration measurements $(\mathrm{mm})$ and mode of failure 


\begin{tabular}{|c|c|c|c|c|}
\hline 1 week & 2 weeks & 16 weeks & 20 weeks & 24 weeks \\
\hline Thermo-cycled & Thermo-cycled & Thermo-cycled & Thermo-cycled & Thermo-cycled \\
\hline \begin{tabular}{|c|}
3.4 \\
3.9 \\
4.6 \\
7.4 \\
8.5 \\
7.34 \\
9.4 \\
10.2 \\
17.2 \\
17.5
\end{tabular} & $\begin{array}{c}6.4 \\
6.7 \\
9.9 \\
10.9 \\
3.0+4.2 \\
4.3+2.8 \\
15.4 \\
17.0 \\
17.3 \\
19.0\end{array}$ & $\begin{array}{c}5.1 \\
5.5 \\
6.2 \\
6.3 \\
6.35 \\
\mathbf{3 . 3}+2.4 \\
\mathbf{3 . 4}+\mathbf{2 . 4} \\
\mathbf{4 . 4}+\mathbf{2 . 0} \\
\mathbf{5 . 2}+2.1 \\
\mathbf{5 . 8}+\mathbf{3 . 4}\end{array}$ & $\begin{array}{c}2.1 \\
3.3 \\
3.4 \\
3.4 \\
3.4 \\
4.3 \\
4.4 \\
2.0+3.0 \\
3.4+1.5 \\
3.4+2.5\end{array}$ & $\begin{array}{c}5.2 \\
6.1 \\
8.3 \\
8.7 \\
9.2 \\
4.2+3.2 \\
10.2 \\
12.1 \\
12.7 \\
15.2\end{array}$ \\
\hline Load cycled & Load cycled & Load cycled & Load cycled & Load cycled \\
\hline \begin{tabular}{|c|}
3.0 \\
3.8 \\
4.5 \\
8.3 \\
10.2 \\
7.3 \\
10.1 \\
11.8 \\
12.9 \\
16.0
\end{tabular} & $\begin{array}{c}6.7 \\
7.2 \\
4.3+1.0 \\
4.3+2.0 \\
6.1+4.3 \\
8.4+2.0 \\
9.3 \\
9.4 \\
11.0 \\
12.3\end{array}$ & $\begin{array}{c}3.7 \\
4.5 \\
5.1 \\
4.2+2.3 \\
4.3+3.1 \\
4.6+2.0 \\
5.0 \\
6.0 \\
6.3 \\
7.3\end{array}$ & $\begin{array}{c}2.3 \\
2.3 \\
3.2 \\
3.8 \\
4.3 \\
4.4 \\
4.5 \\
5.4 \\
5.4 \\
3.4+2.1\end{array}$ & \begin{tabular}{|c|}
8.4 \\
9.5 \\
9.8 \\
9.8 \\
10.0 \\
11.0 \\
11.4 \\
12.2 \\
12.3 \\
12.8
\end{tabular} \\
\hline $\begin{array}{l}\text { Thermo/Load } \\
\text { cycled }\end{array}$ & $\begin{array}{l}\text { Thermo/Load } \\
\text { cycled }\end{array}$ & $\begin{array}{c}\text { Thermo/Load } \\
\text { cycled }\end{array}$ & $\begin{array}{c}\text { Thermo/Load } \\
\text { cycled }\end{array}$ & $\begin{array}{c}\text { Thermo/Load } \\
\text { cycled }\end{array}$ \\
\hline $\begin{array}{c}4.3+2.1 \\
4.3+2.3 \\
5.4 \\
6.4 \\
8.3 \\
9.9 \\
10.1 \\
10.2 \\
10.3 \\
12.1\end{array}$ & $\begin{array}{c}5.5 \\
9.8 \\
10.1 \\
12.8 \\
3.9+5.8 \\
5.0+5.5 \\
13.4 \\
17.0 \\
18.0 \\
19.0\end{array}$ & $\begin{array}{c}3.4 \\
5.0 \\
5.1 \\
5.8 \\
6.3 \\
8.2 \\
3.2+2.1 \\
4.3+5.0 \\
7.3+2.3 \\
9.4\end{array}$ & $\begin{array}{c}2.0 \\
4.9 \\
5.4 \\
6.3 \\
3.2+1.1 \\
3.8+2.1 \\
4.3+2.7 \\
4.3+3.1 \\
4.5+2.0 \\
5.1+2.0\end{array}$ & $\begin{array}{l}4.4 \\
5.7 \\
6.7 \\
6.9 \\
8.7 \\
9.4 \\
9.6 \\
9.9 \\
11.5 \\
16.8\end{array}$ \\
\hline
\end{tabular}

Cohesive failure: ${ }^{\bullet} \quad$ Adhesive failure: $\bullet$

Table 5.45 : $\mathrm{Z100}$ dye penetration measurements $(\mathrm{mm})$ and mode of failure 


\begin{tabular}{|c|c|c|c|}
\hline \multirow{2}{*}{ Week } & Specimens & \multicolumn{2}{|c|}{ Failures } \\
\cline { 2 - 4 } & Adhesive & Cohesive \\
\hline \multirow{3}{*}{1} & Thermo-cycled & 10 & 0 \\
& Load cycled & 10 & 0 \\
& Thermo/Load cycled & 10 & 0 \\
\hline \multirow{2}{*}{2} & Thermo-cycled & 0 & 10 \\
& Load cycled & 1 & 9 \\
& Thermo/Load cycled & 0 & 10 \\
\hline \multirow{2}{*}{16} & Thermo-cycled & 0 & 10 \\
& Load cycled & 0 & 10 \\
& Thermo/Load cycled & 0 & 10 \\
\hline \multirow{2}{*}{20} & Thermo-cycled & 0 & 10 \\
& Load cycled & 1 & 9 \\
& Thermo/Load cycled & 0 & 10 \\
& Thermo-cycled & 6 & 4 \\
& Load cycled & 2 & 6 \\
\hline & Thead cycled & 4 & 0 \\
\hline
\end{tabular}

Table 5.46 : Silux Plus mode of failure 


\begin{tabular}{|c|c|c|c|}
\hline \multirow{2}{*}{ Week } & \multirow{2}{*}{ Specimens } & \multicolumn{2}{|c|}{ Failures } \\
\hline & & Adhesive & Cohesive \\
\hline \multirow{3}{*}{1} & Thermo-cycled & 5 & 5 \\
\hline & Load cycled & 5 & 5 \\
\hline & Thermo/Load cycled & 8 & 2 \\
\hline \multirow{3}{*}{2} & Thermo-cycled & 4 & 6 \\
\hline & Load cycled & 4 & 6 \\
\hline & Thermo/Load cycled & 4 & 6 \\
\hline \multirow{3}{*}{16} & Thermo-cycled & $\mathbf{0}$ & 10 \\
\hline & Load cycled & 4 & 6 \\
\hline & Thermo/Load cycled & 1 & 9 \\
\hline \multirow{3}{*}{20} & Thermo-cycled & 0 & 10 \\
\hline & Load cycled & 0 & 10 \\
\hline & Thermo/Load cycled & 0 & 10 \\
\hline \multirow{3}{*}{24} & Thermo-cycled & 4 & 6 \\
\hline & Load cycled & 10 & $\mathbf{0}$ \\
\hline & Thermo/Load cycled & 7 & 3 \\
\hline
\end{tabular}

Table 5.47 : Z100 mode of failure 


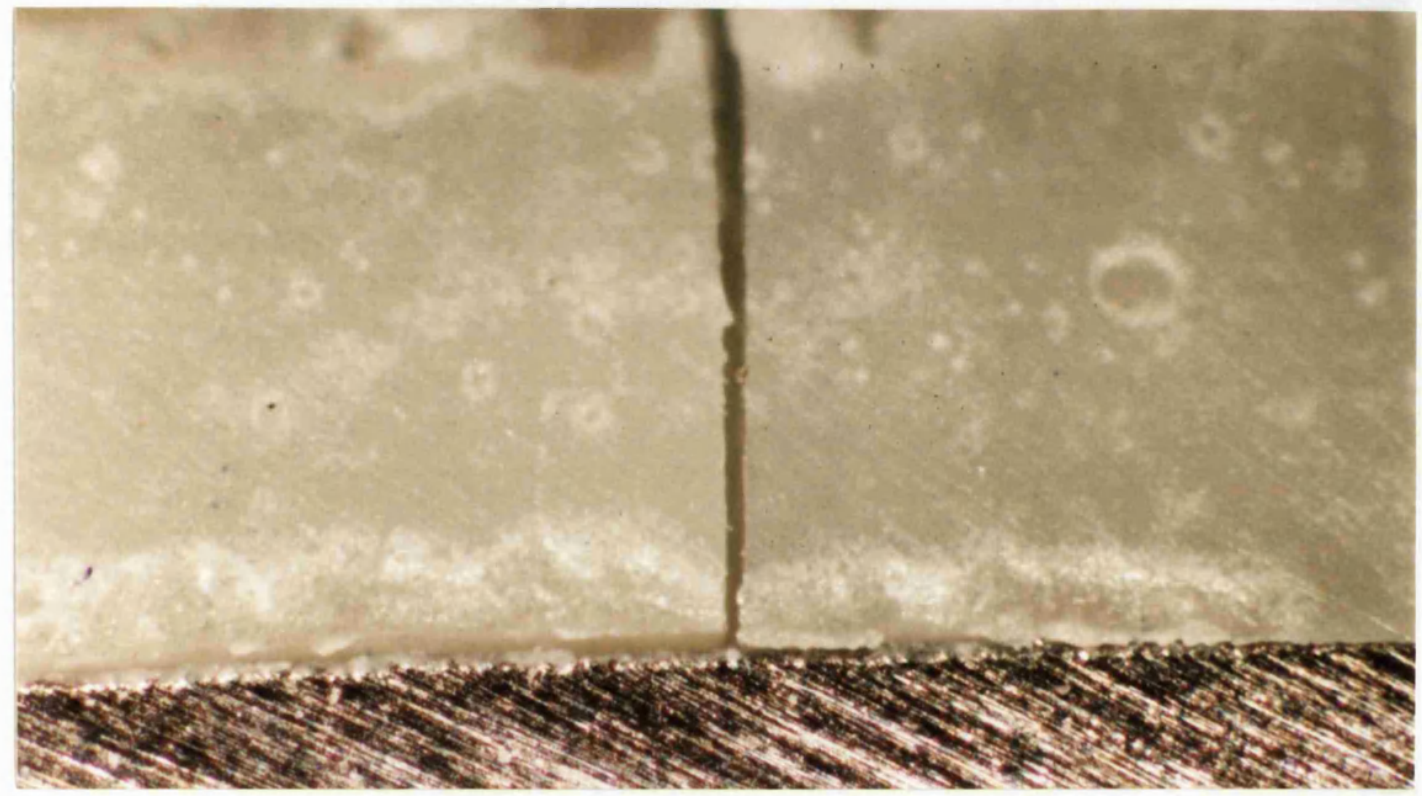

Figure 5.32a : A cohesive failure with the fracture line divided

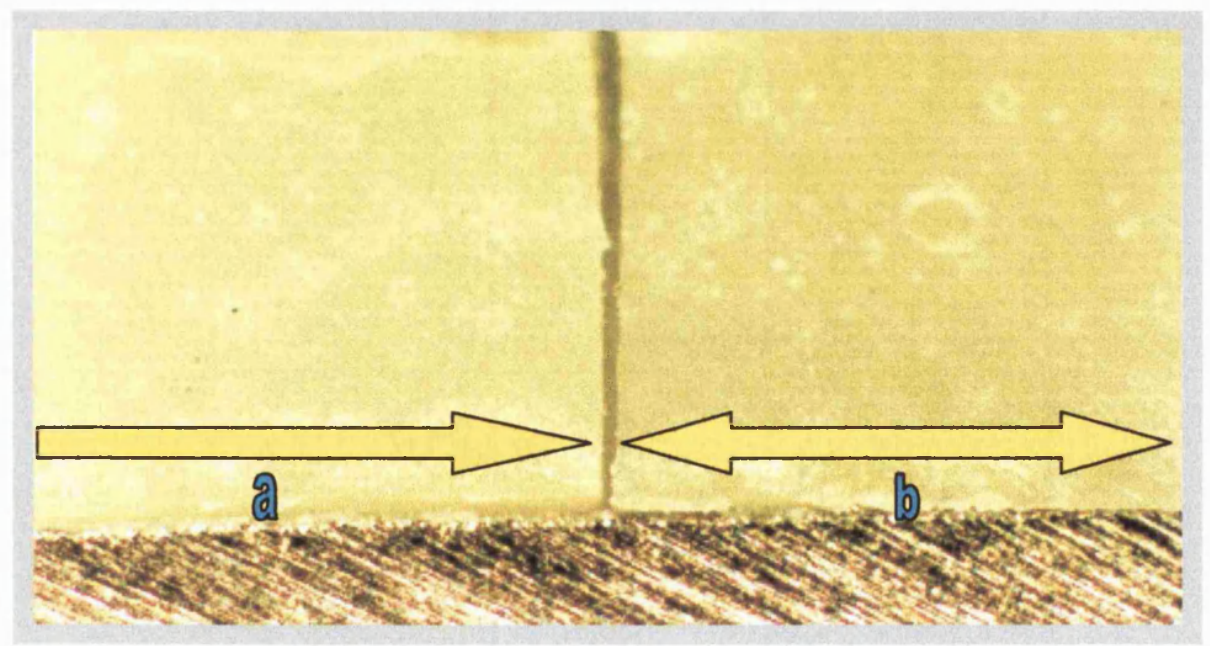

Figure 5.32b : A cohesive failure with the fracture line divided

( $\mathrm{a}$ and $\mathrm{b}$ both represent the lengths of the fracture lines from start to finish as determined when the specimens were examined under a $32 x$ magnification. In this case a extends longer than the margins of the print) 


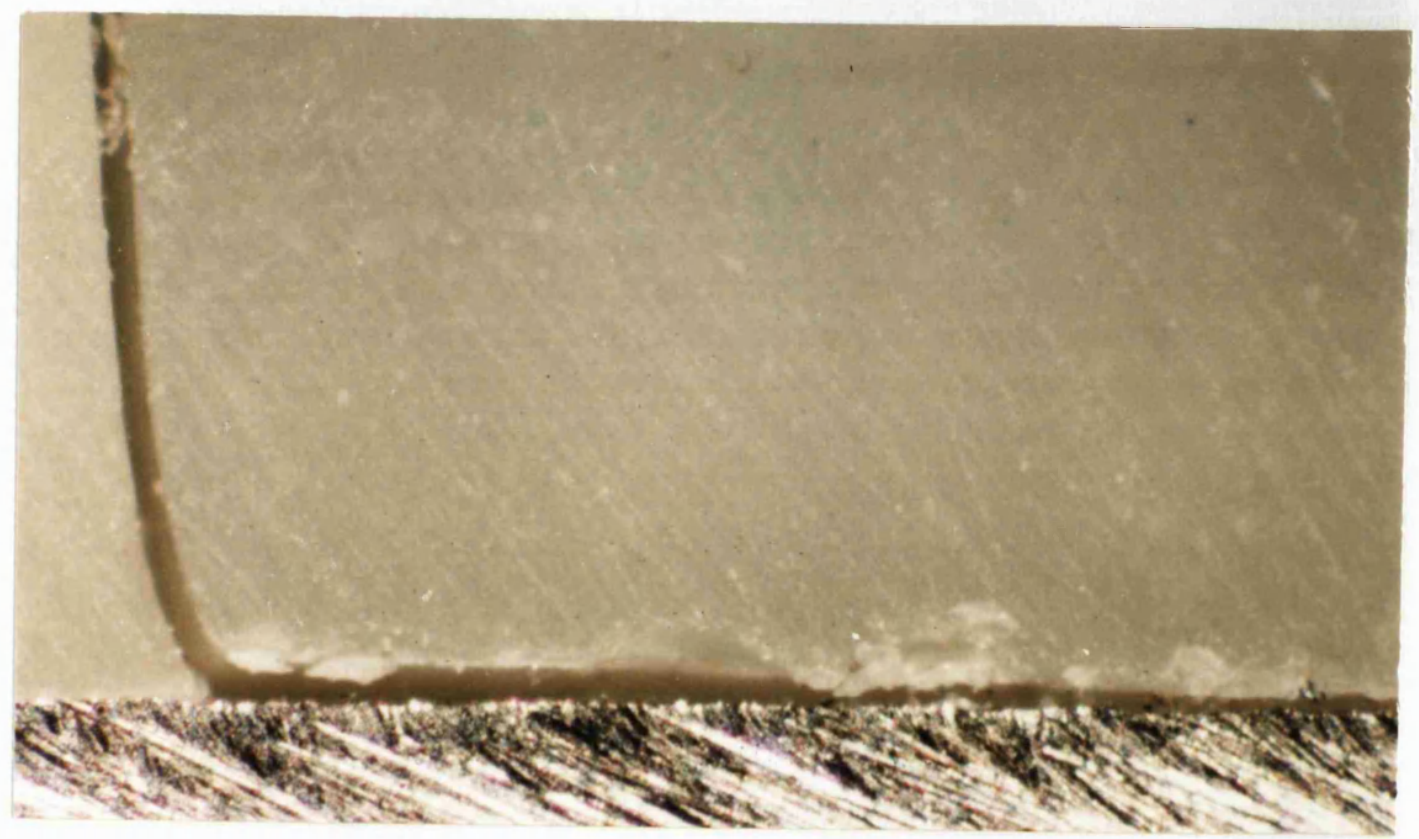

Figure 5.33a : A cohesive failure with a single fracture line

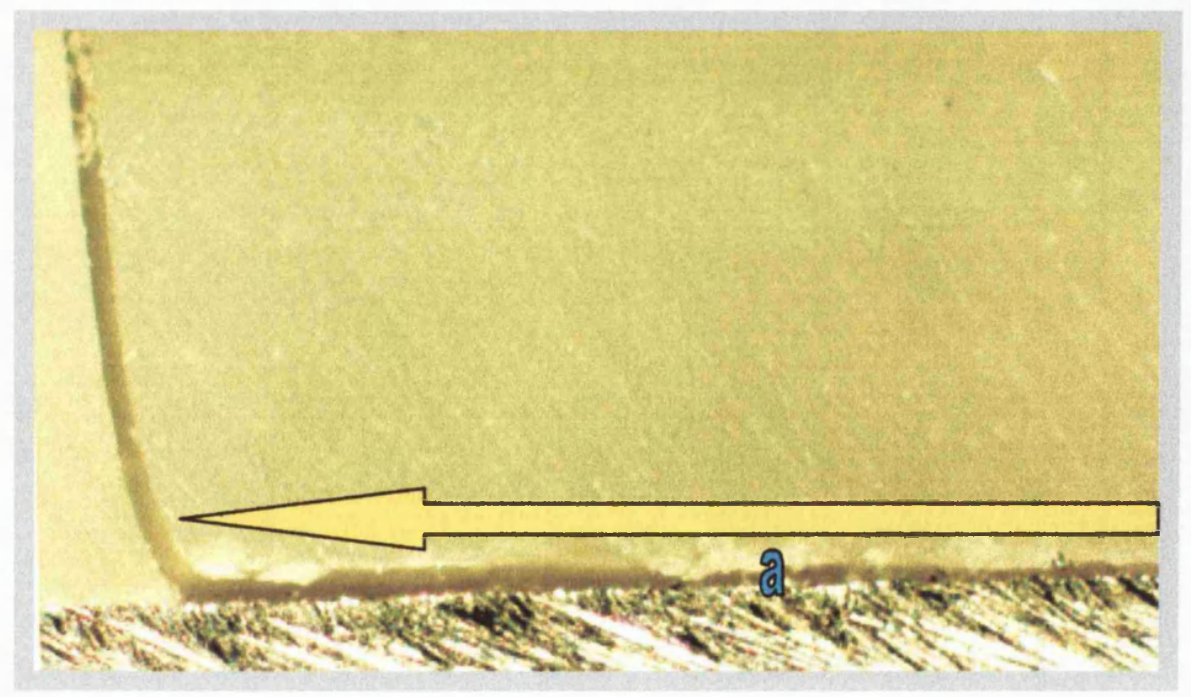

Figure 5.33b : A cohesive failure with a single fracture line

(a represents the length of the fracture line from start to finish as determined when the specimens were examined under a $32 \mathrm{x}$ magnification. In this case a extends longer than the margins of the print) 


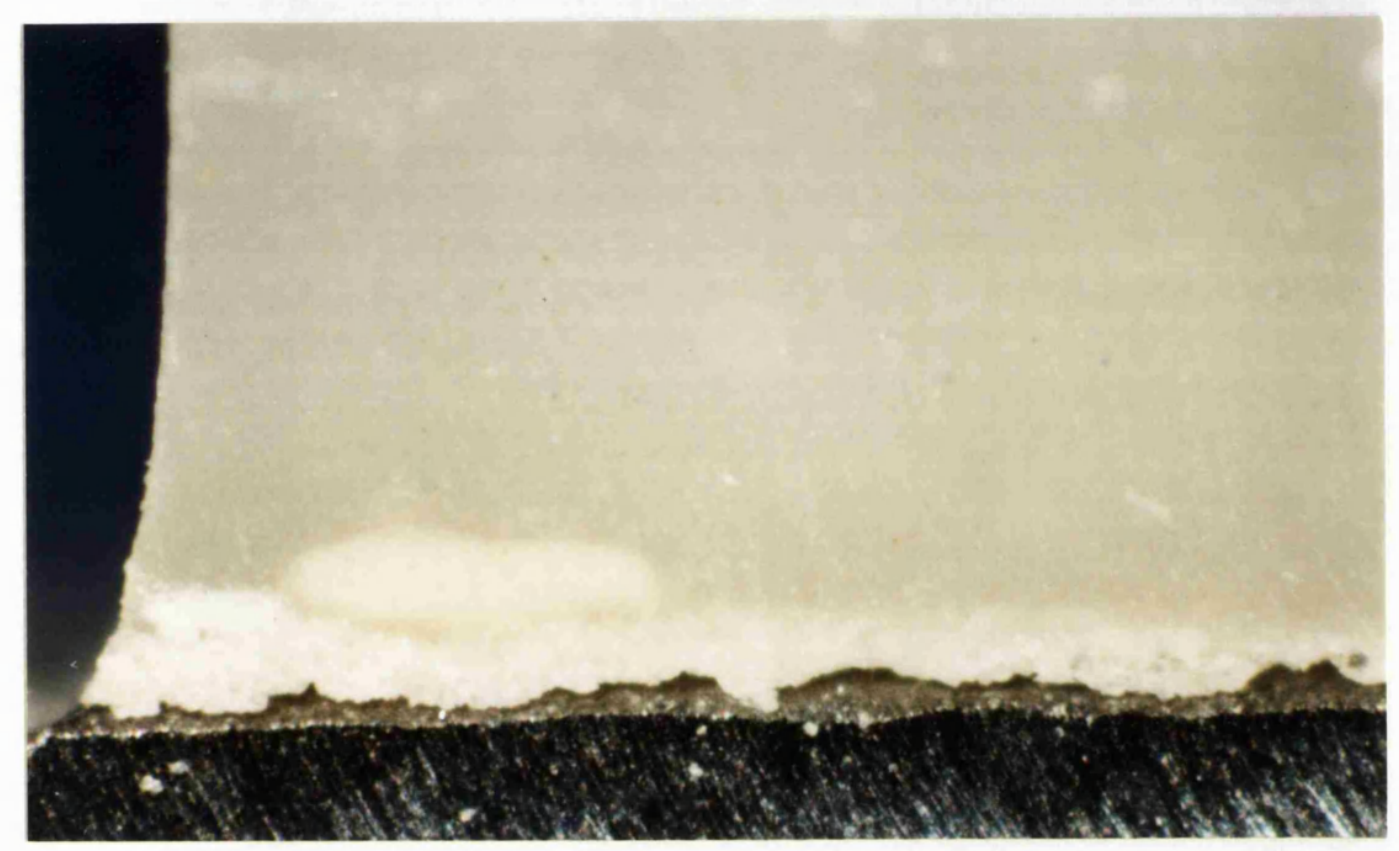

Figure 5.34a : An adhesive failure

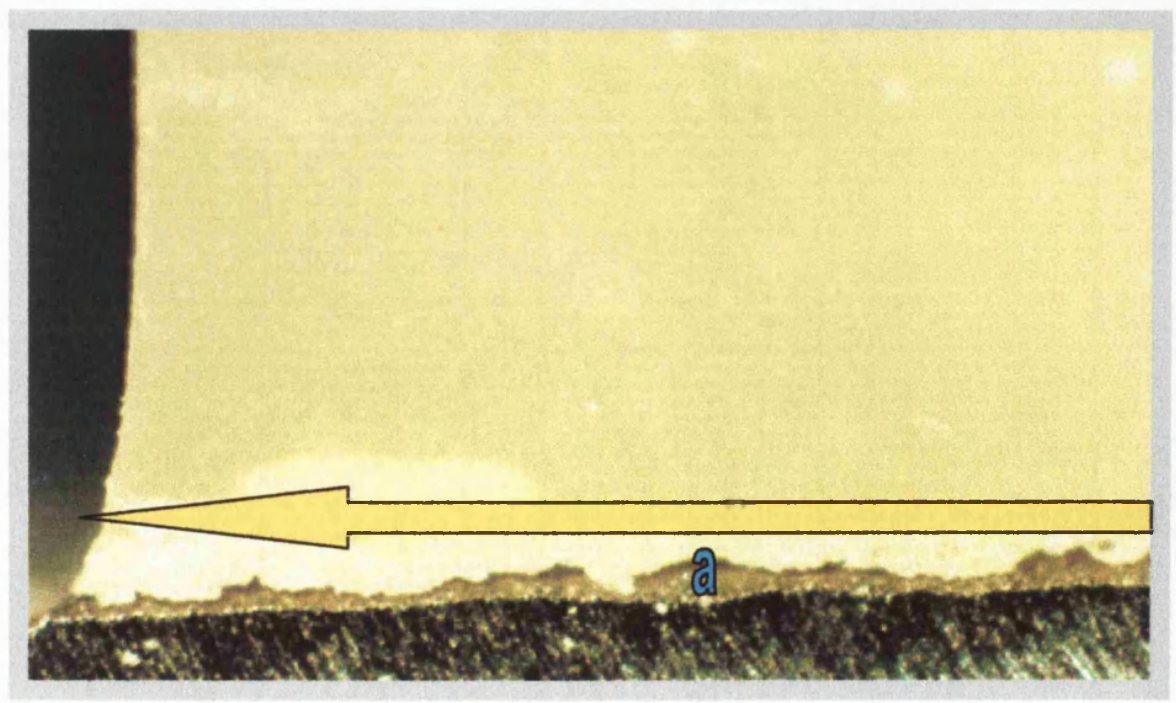

Figure 5.34b : An adhesive failure

(a represents the extent of the fracture line from start to finish as determined when the specimens were examined under a $32 \mathrm{x}$ magnification. In this case a extends longer than the margins of the print) 


\subsubsection{SEM ANALYSIS}

The scanning electron micrographs showed two distinctive appearances for the two materials. The microfine material (Silux Plus) showed large particles of some approximating $20-30 \mu \mathrm{m}$ and fractures appeared to go along the line of the prepolymerised blocks. The hybrid (Z100) had much smaller filler particles and appeared much more amorphous in character. At some specimens some porosity was observed. Over the duration of the experiment there was little general change in the samples of Silux Plus. In all cases the fracture appeared to follow the line of the surface of the prepolymerised blocks. As the duration of the experiment was extended, there was some indication of prepolymerised blocks becoming separated from the resin matrix. The Z100 specimens showed little change but at the later stages there was some early indication of lamination as seen in Figures $5.40 \mathrm{a}+\mathrm{b}$. 


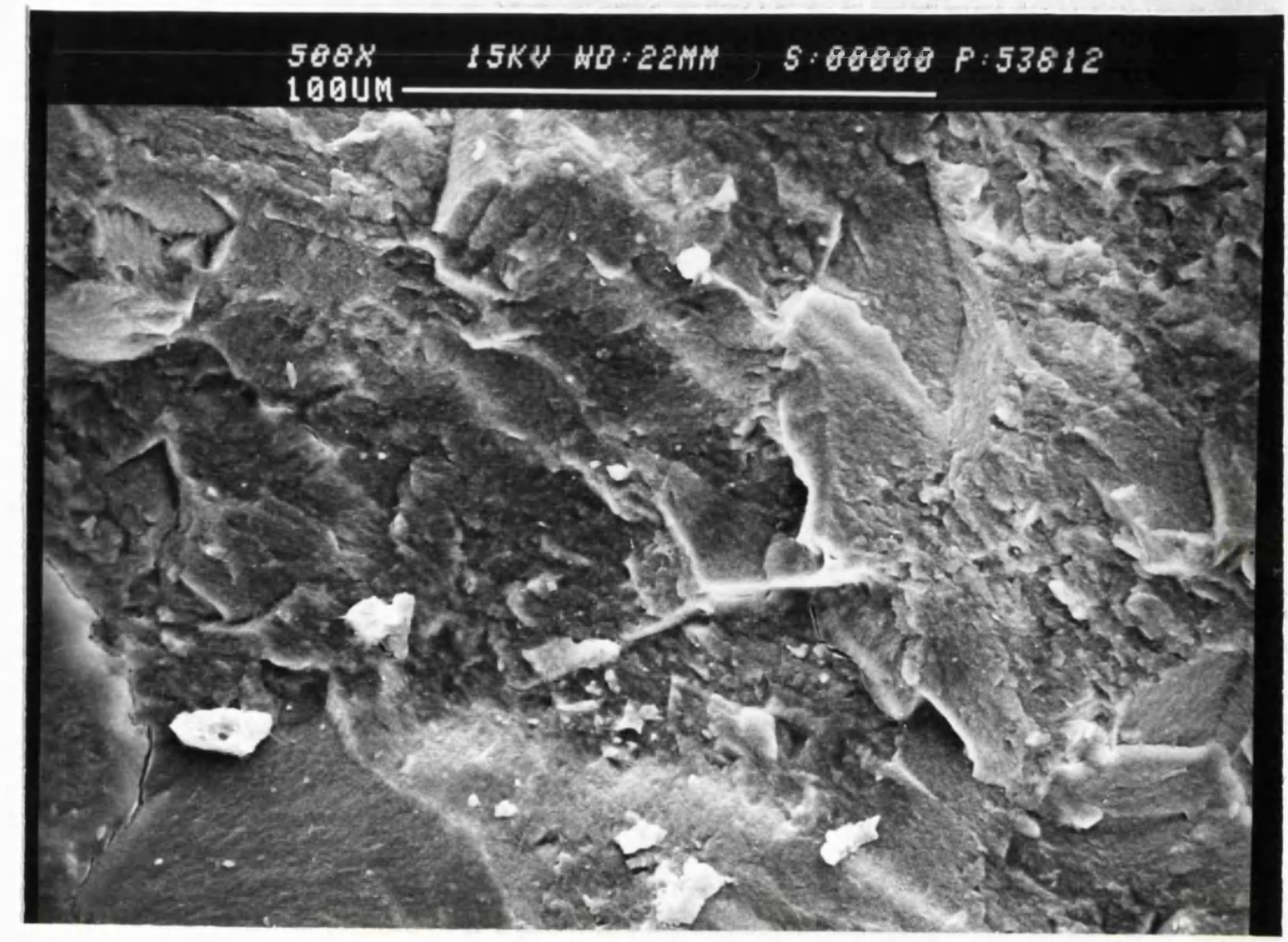

Figure 5.35a : Silux Plus 2 weeks thermo-cycled group

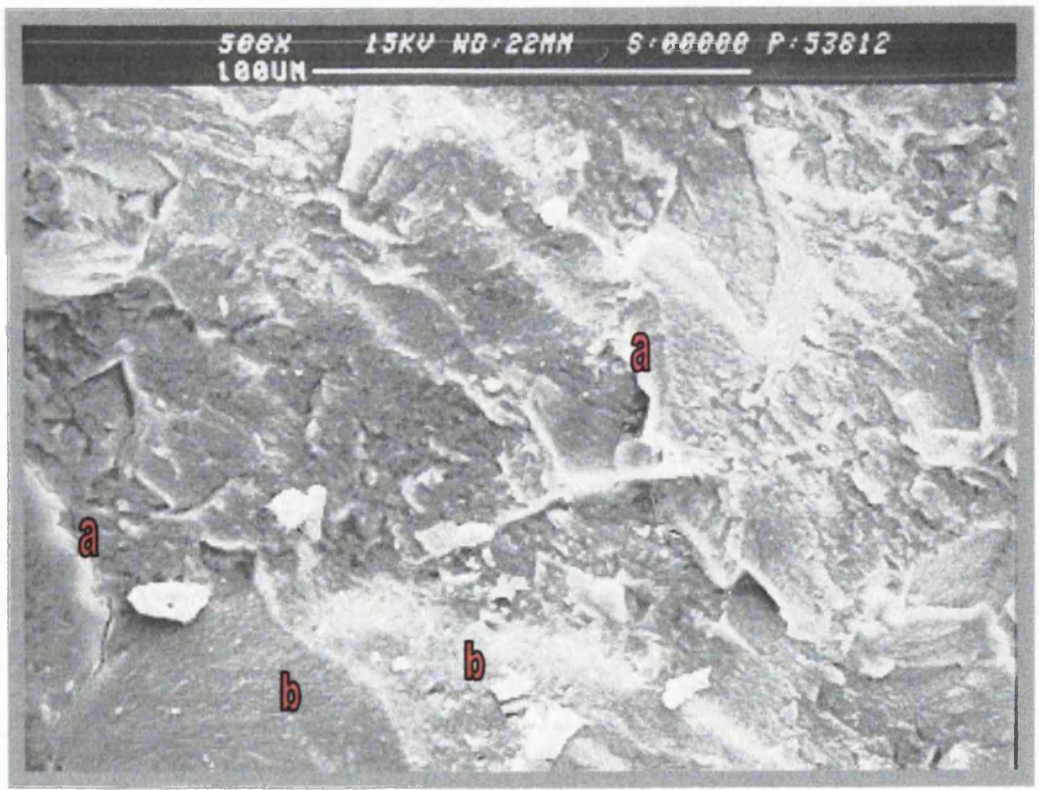

Figure 5.35b : Silux Plus 2 weeks thermo-cycled group

(Field of view: $275 \mu \mathrm{m}$. Magnification: 508x. This micrograph shows the fracture surface of Silux Plus at 2 weeks: thermo-cycled group. The surface is irregular as there are a number of interfaces (a) with clearly defined margins. These are likely to be the prepolymerised blocks $(b))$. 


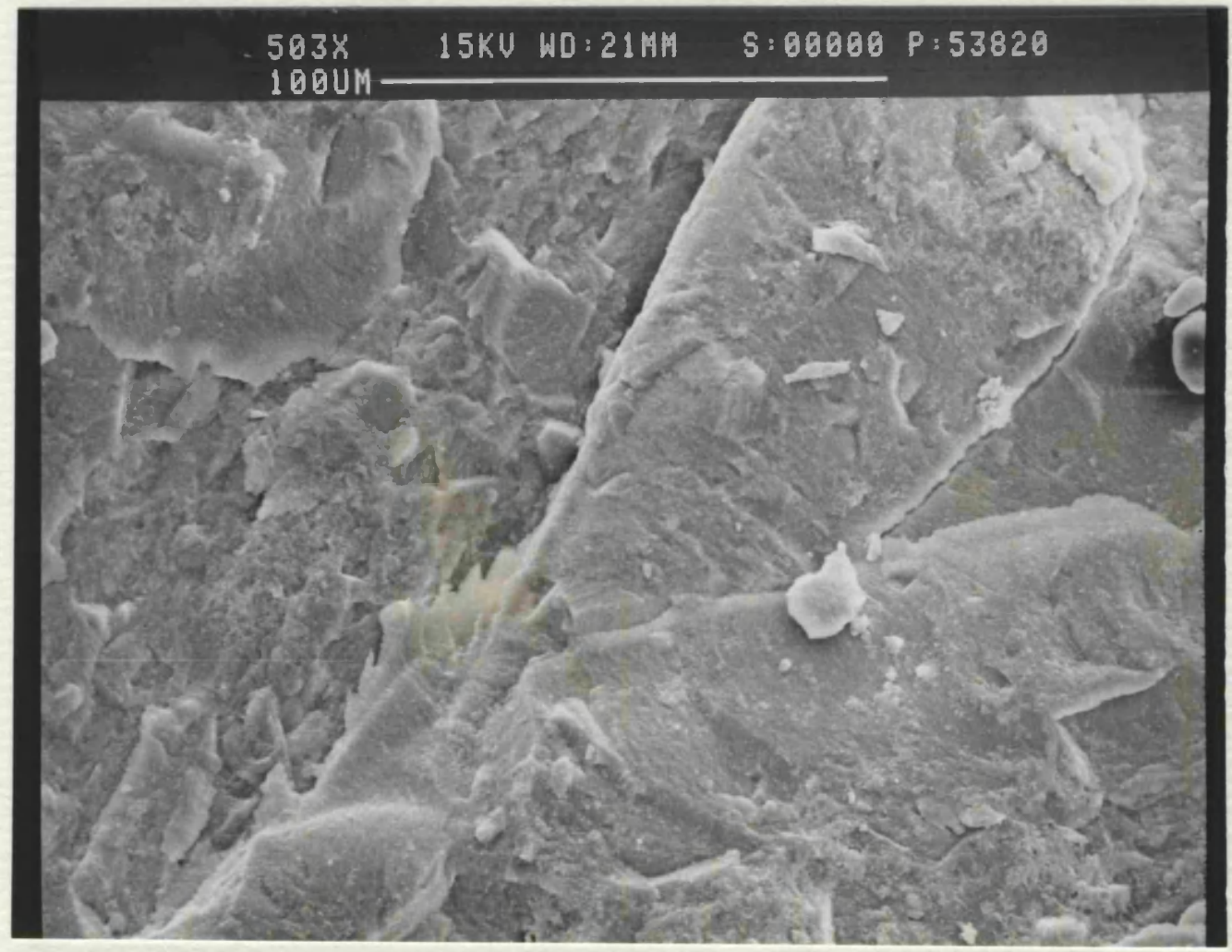

Figure 5.36a : Silux Plus 2 weeks thermo/load cycled group

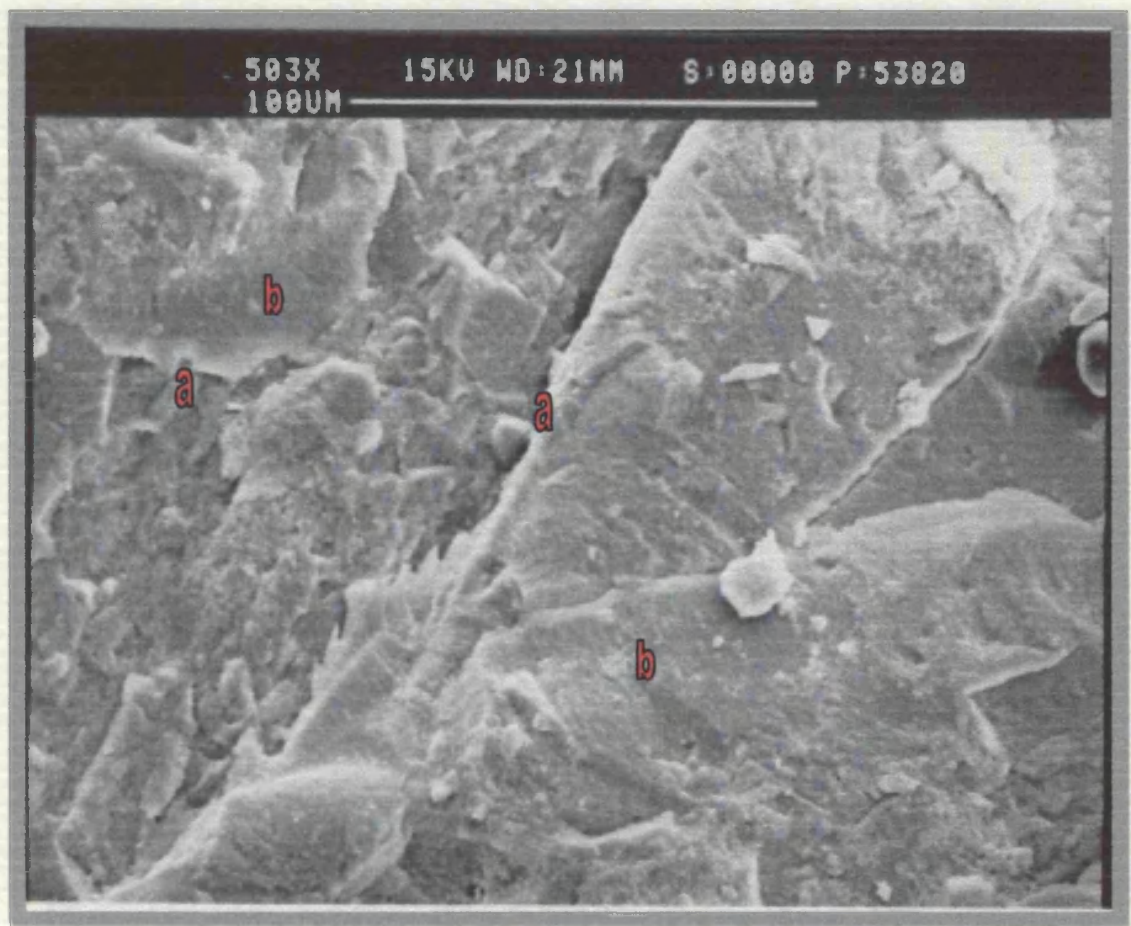

Figure 5.36b : Silux Plus 2 weeks thermo/load cycled group

(Field of view: $226 \mu \mathrm{m}$. Magnification: 503x. This micrograph shows the fracture surface of Silux Plus at 2 weeks: thermo/load cycled group. Similarly to the 2 weeks thermo-cycled group, the surface is irregular as there are a number of interfaces (a) with clearly defined margins. These are likely to be the prepolymerised blocks (b)). 


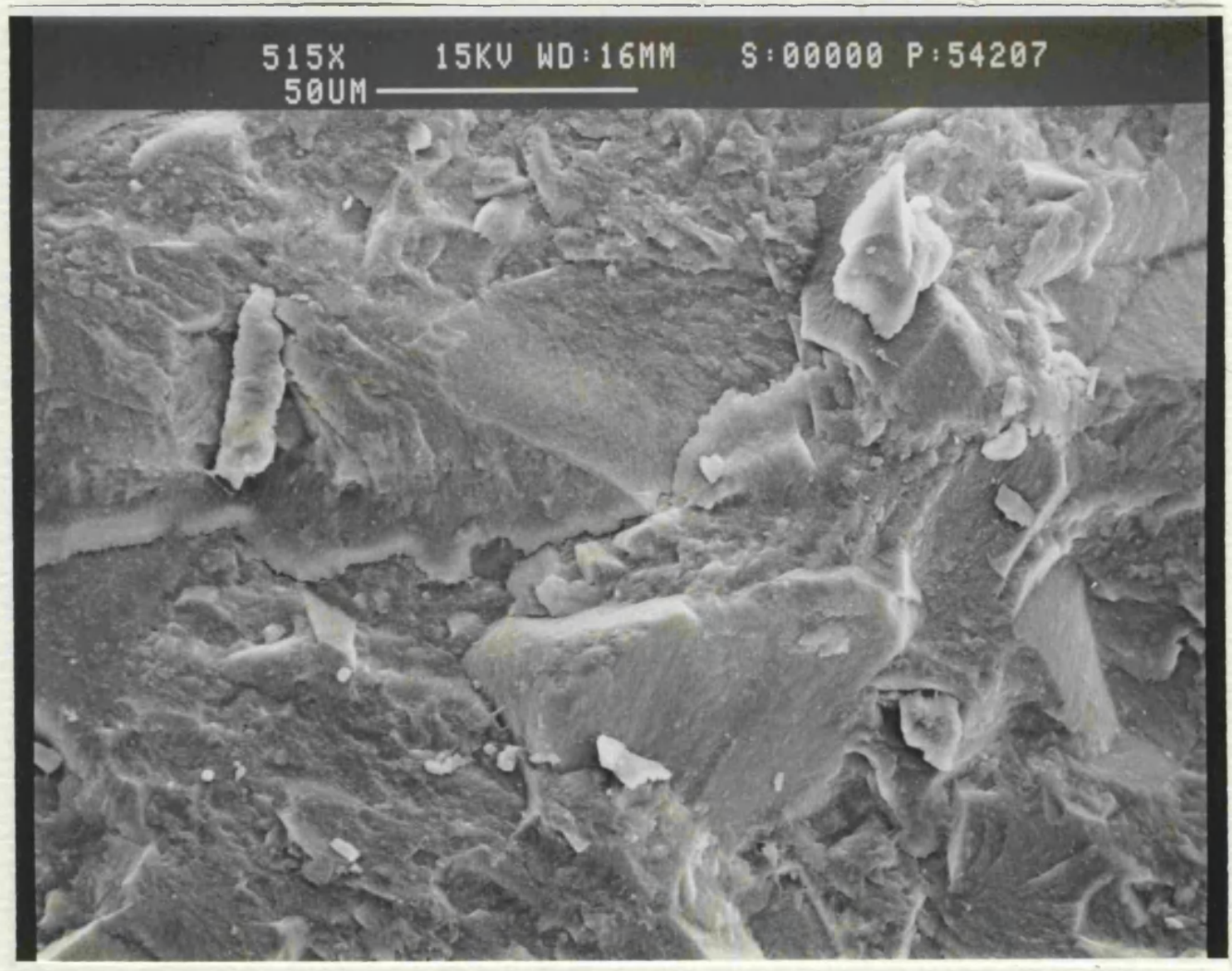

Figure 5.37a : Silux Plus 20 weeks thermo-cycled group

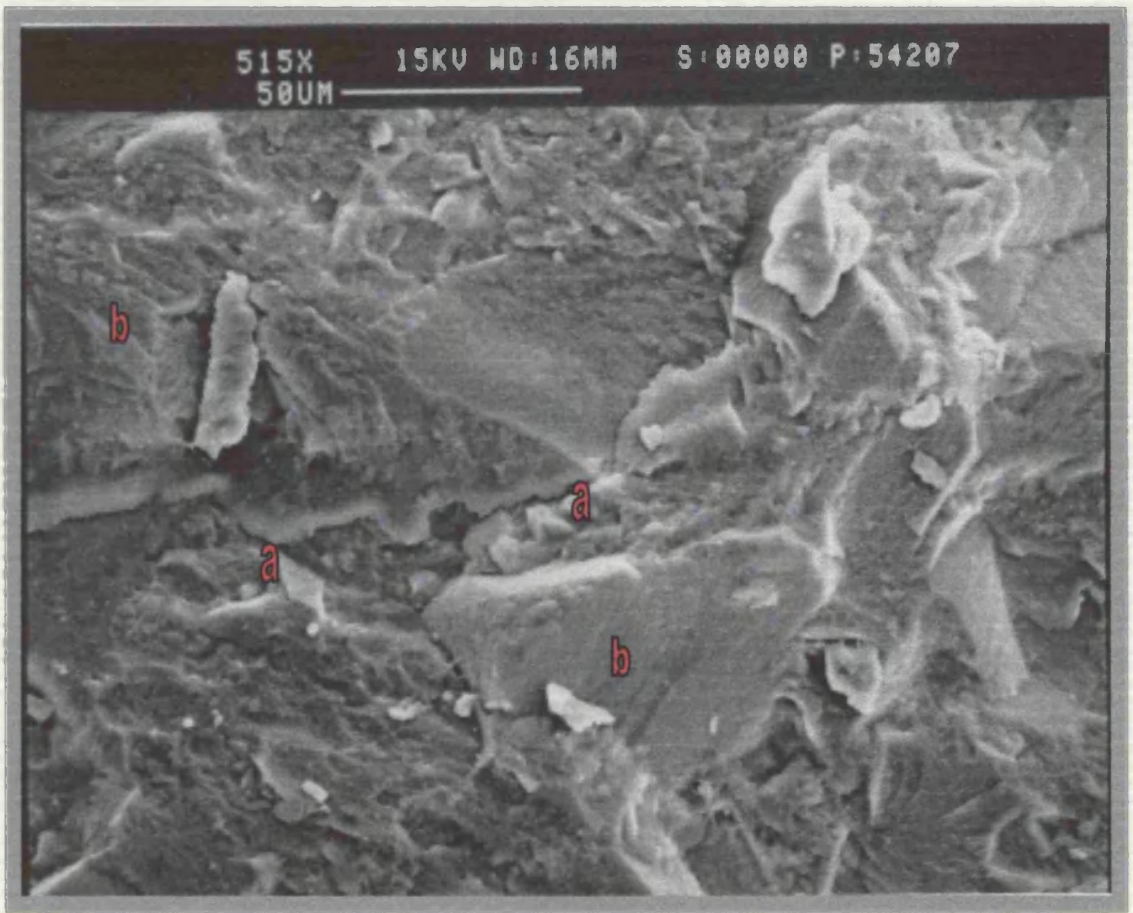

Figure 5.37b : Silux Plus 20 weeks thermo-cycled group

(Field of view: $439 \mu \mathrm{m}$. Magnification: $515 \mathrm{x}$. This micrograph shows the fracture surface of Silux Plus at 20 weeks: thermo-cycled group. The surface is very irregular as there are a number of interfaces (a) with clearly defined margins. These are likely to be the prepolymerised blocks (b). Some separation of the prepolymerised blocks is noted). 


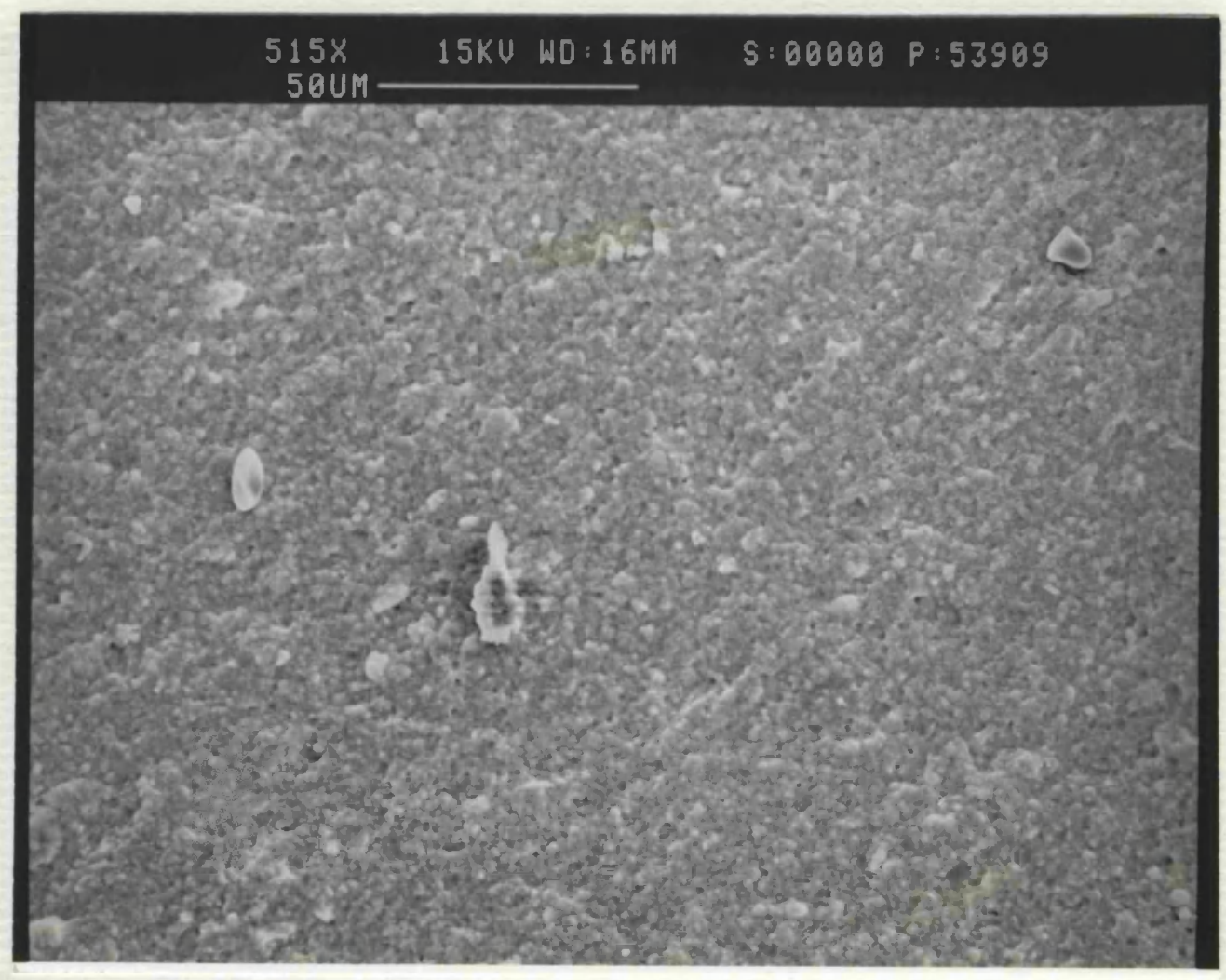

Figure 5.38a : Z100 2 weeks thermo-cycled group

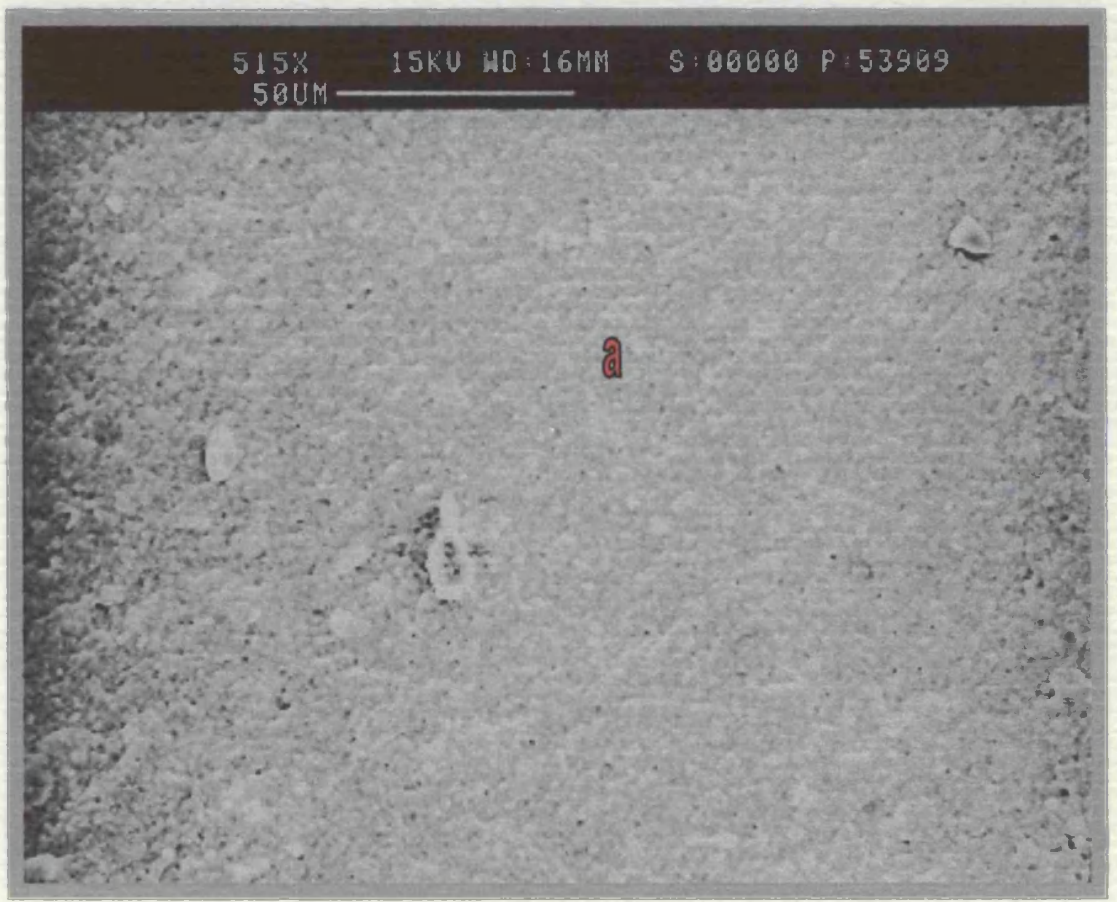

Figure 5.38b : Z100 2 weeks thermo-cycled group

(Field of view: $219 \mu \mathrm{m}$. Magnification: 515x. This micrograph shows the fracture surface of $\mathrm{Z100}$ at 2 weeks: thermo-cycled group. There is generally an amorphous structure (a). There is little evidence of fracture pattern). 


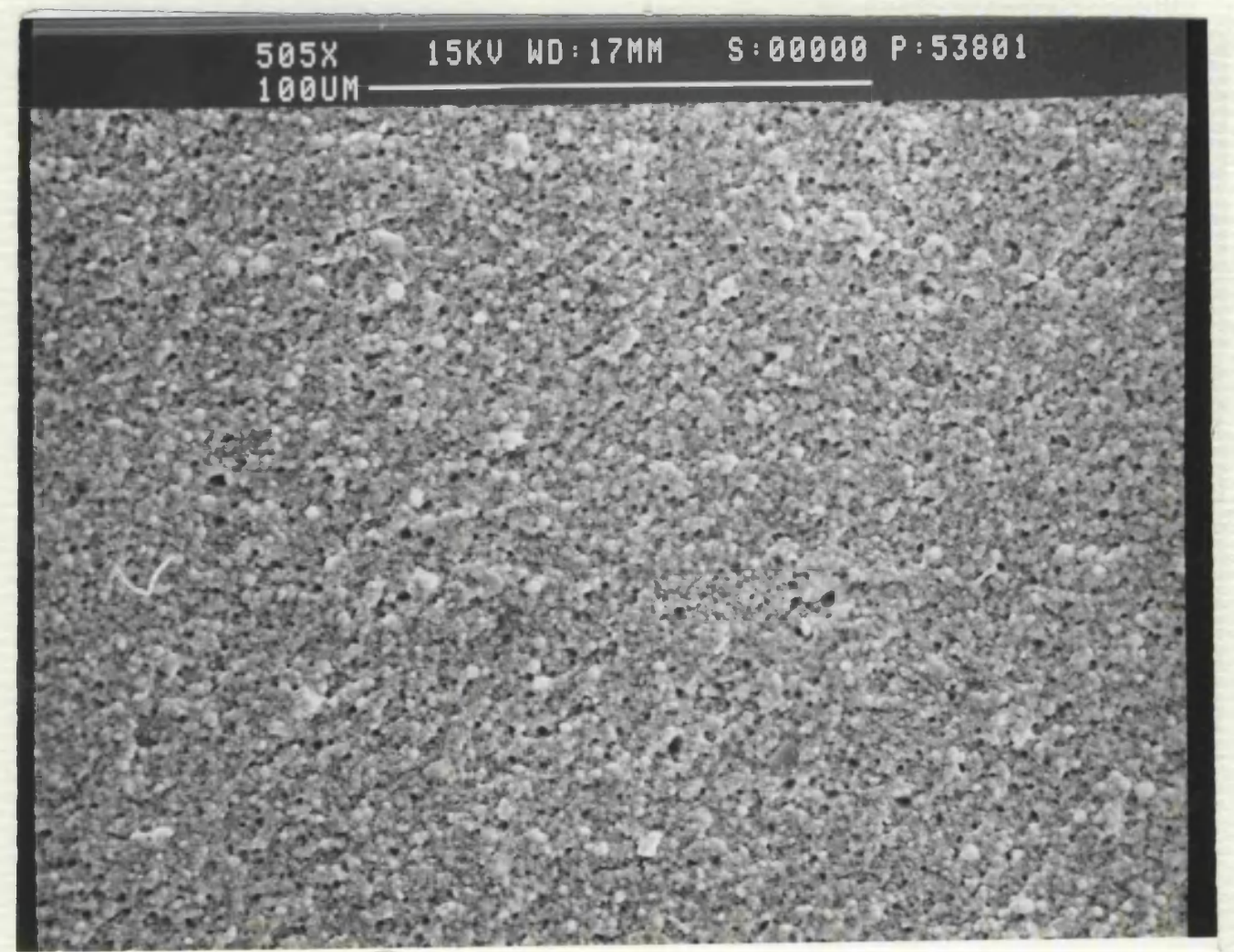

Figure 5.39a : Z100 2 weeks thermo/load cycled group

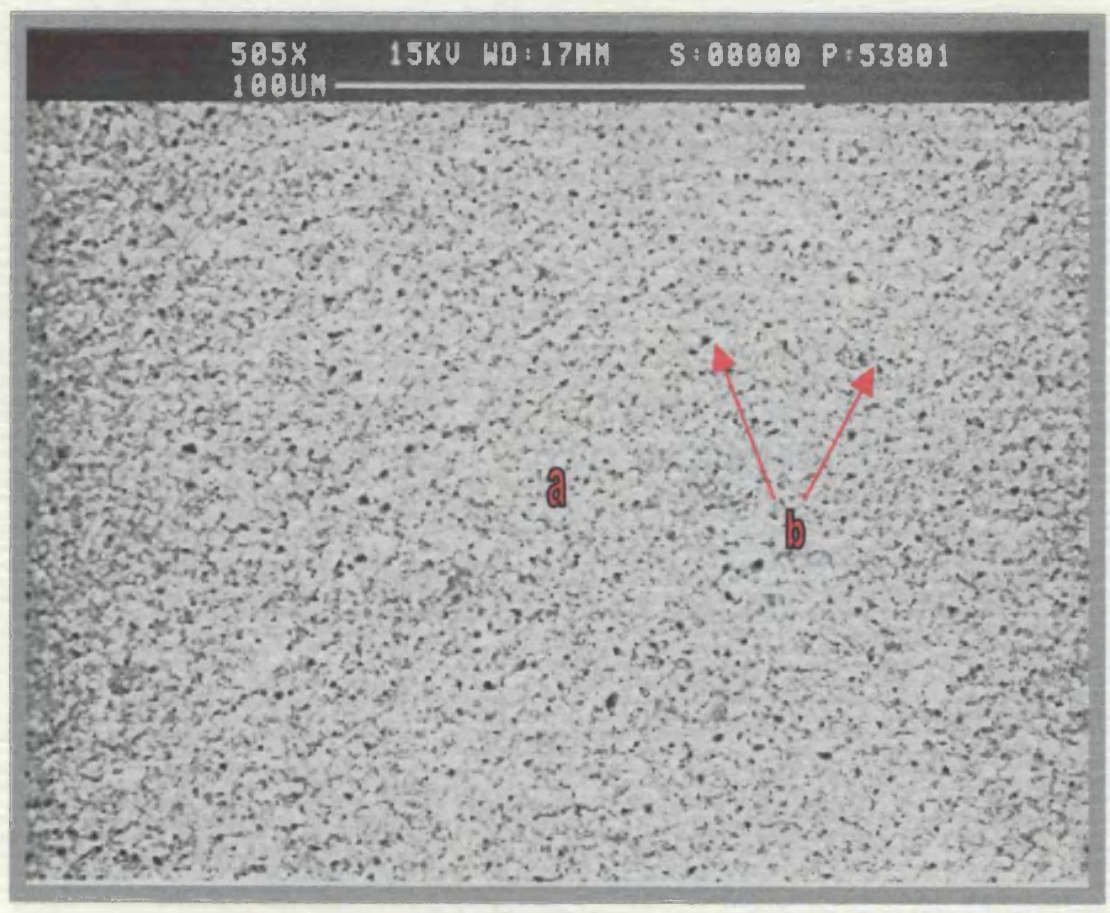

Figure 5.39b : Z100 2 weeks thermo/load cycled group

(Field of view: $223 \mu \mathrm{m}$. Magnification: 505x. This micrograph shows the fracture surface of $\mathrm{Z100}$ at 2 weeks: thermo/load cycled group. There is generally an amorphous structure (a). There is little evidence of fracture pattern. Note porocity (black dots) (b). There is potential for water absorption in the pores). 


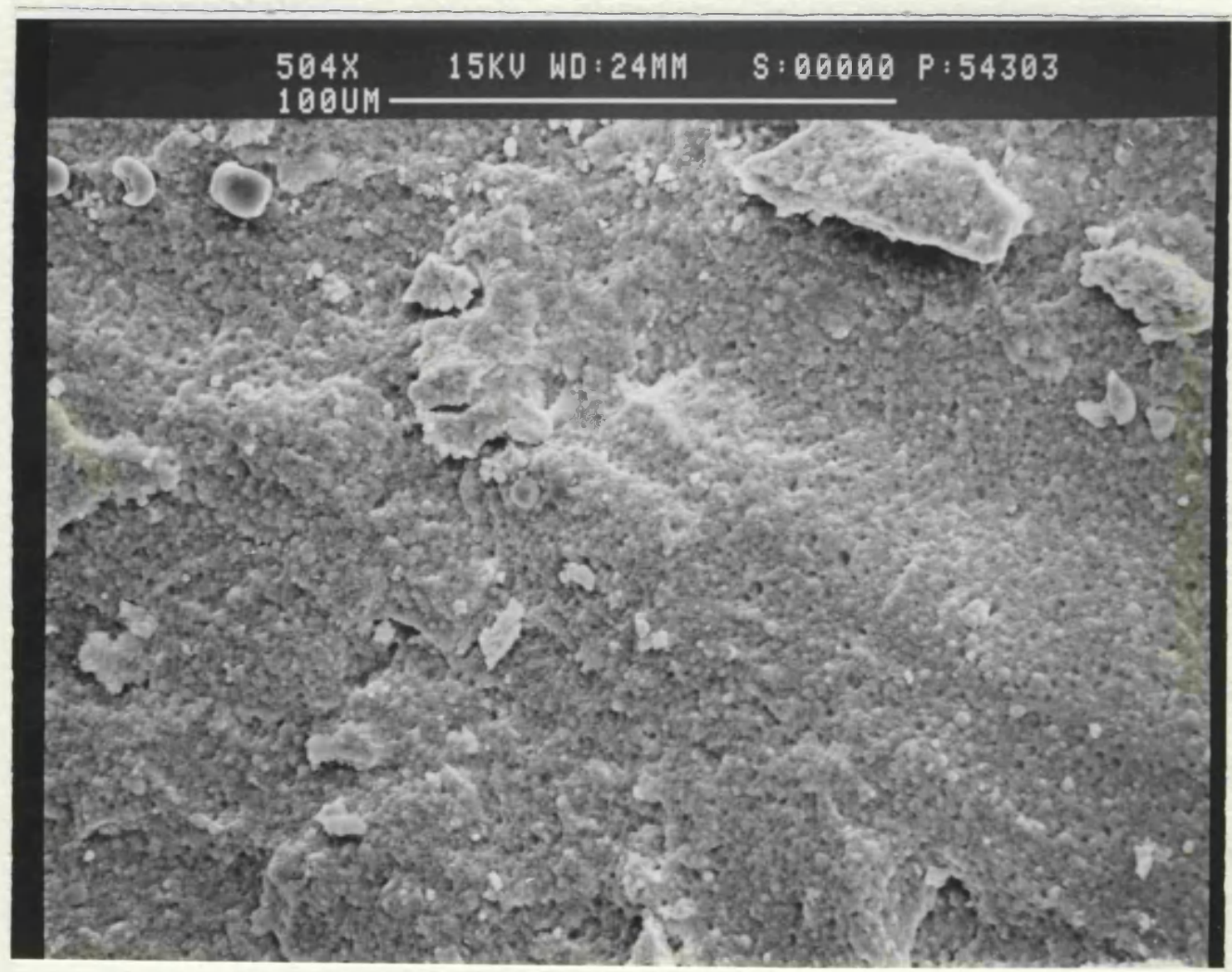

Figure 5.40a : Z100 20 weeks thermo/load cycled group

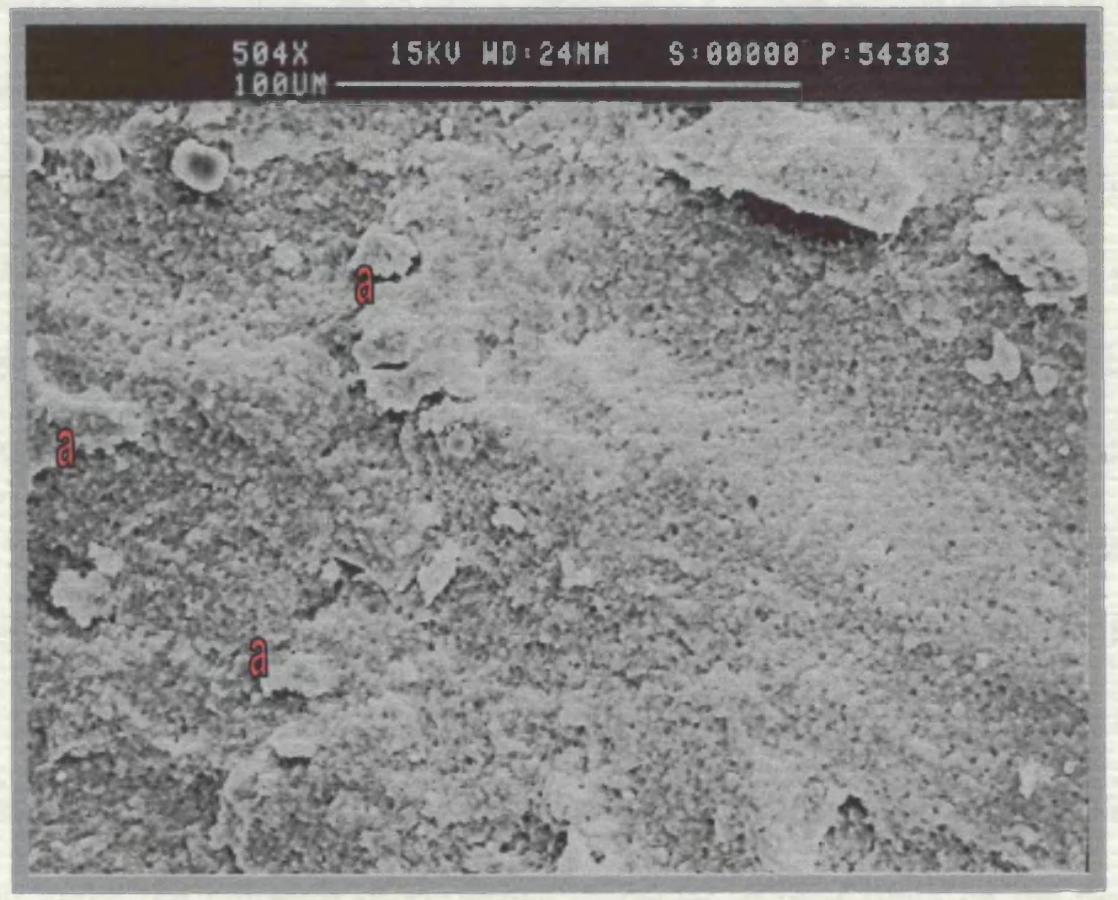

Figure 5.40b : Z100 20 weeks thermo/load cycled group

(Field of view: $226 \mu \mathrm{m}$. Magnification: $504 \mathrm{x}$. This micrograph shows the fracture surface of Z100 at 20 weeks: thermo-cycled group. There is some lamination of the surface (a) and apparent flake like appearance). 


\section{DISCUSSION}

\subsection{INTRODUCTION}

A successful partial denture should replace missing tissues with materials that have similar aesthetic characteristics. Therefore, metal and polymer components are often used together to address the design requirements of an RPD. Under clinical conditions, polymer veneers on an RPD are likely to fail due to wear, fracture or interfacial failure (Hobkirk et al 1989). To reduce the incidence of interfacial failure, designs incorporating both mechanical retention and chemical adhesive techniques have been used to improve the retention of the polymer veneers to the metal components. Macromechanical retention devices prove problematic, due to the extra bulk that they incorporate in the design. Nowadays, these retentive systems are being superseded by the use of chemical adhesives in association with micromechanical retentive preparation of the metal surface.

While initial strength is an obvious requirement for clinical function, the durability of the restorative materials subjected to oral stresses over a period of time is equally important. The modes of failure mentioned earlier are reinforced in the clinical environment by a fatigue process caused by the cyclical application of occlusal forces upon the denture. Materials are subjected to mechanical, thermal and chemical processes in the mouth during routine drinking and eating, which by their cyclical nature invariably induce fatigue. Fatigue failure may be explained by the development of microscopic cracks in areas of stress concentration. With continued loadings, these cracks fuse together to a propagating main crack which weakens the material employed. Catastrophic failure can result from a final loading cycle that exceeds the capacity of the remaining unaffected material to absorb the applied energy (Wiskott et al 1995). Composite resins tend to deform under the influence of the above processes, putting strain on the resin matrix. 
Cracks may begin to form in this matrix which eventually grow until failure has occurred due to the continued opening in the crack tip region (Braem et al 1994a).

Theoretically, a composite resin material with lower elastic modulus, when bonded to metal, may survive longer as a veneering resin in areas where flexural forces predominate. The lower elastic modulus would allow greater flexibility of the veneer and impose less stress upon the interfacial bond. An adhesive bond that integrates well with both the metal and resin components opposes the development of localised areas of stress concentration at the interface, therefore prolonging the clinical service of the prosthesis. It is for this reason that the current study was initiated since the performance of an "adhesive" bonding system used in conjunction with either a highly filled or resin rich composite would be of clinical interest.

\subsection{COMPOSITE MATERIALS}

\subsubsection{ADHESIVE RESIN}

Cesead is a three element light cured composite system which has been designed for crown and bridge use. The opaque primer contains a metal-adhesive phosphate monomer (10-MDP) that is reported to bond to the alloy surface. The opaque resin bonds to the primer. The opaque resin is light-cured, however, it may additionally be cured chemically when in contact with the opaque primer. This allows polymerisation to proceed in the event that light is unable to penetrate through the entire thickness of the resin. The system was used to provide an adhesive interface which may be resistant to hydrolysis and breakdown. Cesead opaque primer and resin were used since various studies have shown that this combination is least vulnerable to thermal and mechanical stresses when bonded to a cobalt-chromium alloy (Yoshida et al 1993 and 1995, Matsumura et al 1996, Yoshida et al 1996 and 1997, Kountouras et al 1999). The provision of a durable adhesive 
interface, in conjunction with the use of two different types of composite might provide an indication of the relative performance of each combination under load. Since only a low concentration of the adhesive phosphate monomer is found in the formulation of the adhesive primer $(<1 \%)$, the retention is probably achieved by micromechanical means. A possible difference in the size of the filler particles compared to that of other adhesive systems may account for the superior performance of Cesead as mentioned earlier.

\subsubsection{COMPOSITE VENEERING RESINS}

The study assessed the effect of using two composites with differing elastic moduli bonded to a metal substructure, on the ultimate failure strength of the laminate after fatigue loading. The two composite resins used, Z100 or Silux Plus, were bonded on the $\mathrm{Co} / \mathrm{Cr}$ surface using a combination of Cesead opaque primer and opaque resin. Silux Plus is a typical microfine composite containing prepolymerised heat cured resin fillers. The highly resin rich composition leads to a lower elastic modulus. Z100 on the other hand is a heavily inorganically filled, hybrid composite and behaves in a brittle fashion having a modulus substantially higher than the microfine. The use of the Cesead composite was rejected as it consisted of a mixture of resins and filler particles that are commonly found in both microfine and hybrid composites. It was felt that it would have been better to compare a specific microfine composite to a specific hybrid that have the same resin components but only differ in their filler components. The difference in the filler content of Silux Plus and Z100 would appear to influence the mechanical properties of the two materials as evaluated during the preliminary study.

Silux Plus has a higher polymer content and lower modulus than the heavily filled Z100. Thus it could be predicted that the two materials would behave differently under load. Highly filled hybrids deform little in function. Developing cracks are likely to pass 
through the matrix along filler interfaces causing incremental failure. In contrast the microfines show more uniform bulk wear under load (Braem et al 1994a). Their differences in mechanical properties and especially their different flexural modulus may also influence the decision as to where these materials are utilised intraorally. Z100 having a higher elastic modulus than Silux Plus, in theory would serve well in areas that are not susceptible to flexural forces but are more prone to wear. On the other hand, Silux Plus with its lower flexural modulus, would in theory perform better in areas susceptible to flexural forces such as clasp surfaces. However, the metal substructure bonded to them, may lead to variation in behaviour and performance of the laminate structure.

The role of adhesive is of prime importance since it facilitates the bonding of two materials having widely different properties and it is probable that major stresses will be set-up at the interface during testing. The test specimens were therefore designed to represent how the materials might be employed intraorally, in an attempt to understand the influence of the components upon the combined structures' mechanical properties.

\subsection{POLYMERISATION CONTRACTION}

During fabrication of the specimens, initial stresses will have been set-up as the resin polymerised, these will have varied for each material. The impact of these stresses upon the interface will have been reduced by the fact that only one surface of the resin spine was bonded to the metal, this left substantial free surface available for polymerisation stress relaxation. However, this configuration does not completely prevent contraction stresses being set-up at the adhesive interface during polymerisation. The Cesead opaque and primer resins are both chemically and light activated. Although Cesead opaque is a dual cured material, since all opaqued specimens were light cured immediately on application, is quite probable that the capacity of the resin to stress relieve would have 
been reduced by early light curing (Krejci and Lutz 1991). Light cured composites and adhesive resins undergo an immediate and rapid polymerisation reaction that limits stress relief more than chemically cured resins, because the materials only exist in a gel stage for a short time. Theoretically, the lower the capacity to stress relieve, the greater will be the contraction stress. The volume change caused by the polymerisation shrinkage to some extent can be compensated by material flowing from the free unbonded surfaces of the restoration. Therefore, the ability of the polymerising composite to flow, plays an important part in the ultimate stress development. As the polymerisation reaction of light cured composites is faster than chemically cured ones it may be suggested that the flow capability is affected by the method of curing and may lead to a difference in the stress levels developed. Therefore, light cured resin composites may generate higher polymerisation shrinkage stresses than the analogous chemically cured composites (Feilzer et al 1993, Davidson and Feilzer 1997). Once though the bonded interface has been exposed to water, this is absorbed and partially compensates for polymerisation contraction (Hansen 1982, Feilzer et al 1990, Schneider et al 1992, Carvalho et al 1996, Davidson and Feilzer 1997). However this does not happen for some time and is diffusion dependant.

Preliminary studies of some of the properties of the two resin composites were undertaken to evaluate differences which might influence the outcome of the main study.

\subsection{WATER SORPTION STUDY}

Long term water immersion tests and associated dye penetration studies, may be used to investigate the deterioration of the adhesive interface. Water sorption and desorption are diffusion processes (Braden et al 1976). They can be described by two physical parameters; the equilibrium water uptake and the diffusion coefficient (Braden and 
Wright 1983). When a composite resin is immersed in water there is a rapid elution of uncured monomer and oligomers. Although complete saturation of the composite requires a substantial time due to the slow diffusion into the cross-linked resin matrix (Pearson 1979), elution appears to be completed more rapidly, within days. Water can percolate into the polymer matrix and is said to occupy space between the main chains and crosslinks, as well filling microvoids created during the polymerisation process (Söderholm 1983, Takahashi et al 1999). The hygroscopic expansion that takes place may compensate for the effects of polymerisation shrinkage at the resin/metal interface and therefore relieve stresses that have been set up (Hansen 1982, Feilzer et al 1990). This may result in a reduction in stress at the interface in the laminate studies investigated here.

Ferracane et al (1995) studied the effect of water conditioning upon the mechanical properties of experimental composites and found that aging for seven days resulted in a significant reduction in the fracture toughness of an experimental microfine composite, while aging beyond this resulted in a reduction for both microfine and hybrid composites. These workers suggested that this reduction in properties is predominately related to the uptake of water by the resin, which in turn causes a swelling of the cross-linked polymer network. This reduces the frictional forces between the polymer chains allowing slippage to occur having a plastising effect. Our results showed that water sorption of the microfine material was substantially greater than of the hybrid. This is in agreement with Pagniano and Johnston (1996) who related the degree of water sorption to the amount of resin in the composite. The solubility showed a similar pattern, with the microfine showing a larger loss of material initially during the first cycle.

Tanaka et al (1991) supported the hypothesis that only a small fraction of unreacted carbon bonds in the set dental composite are present as residual monomer or elutable 
oligomer. Therefore, it can be assumed that these unreacted methacrylate groups remain bound within the composite irrespective of the time being immersed in water.

Overlay dentures are likely to be constantly immersed in a complex solution of saliva. When stored out of the mouth they are immersed in solutions of denture cleansers or water. The study of water sorption provides a guide to the behaviour of resins, however the conditions in the mouth are more complex. The composition of human saliva is very variable, since the secretions of the various salivary glands are affected by intensity and duration of stimulation, medications, diet, age and sex. Both organic and inorganic components can be expected to vary greatly in different individuals and in the same individual under different circumstances (Mandel 1974, Jenkins 1981, Dowd 1999, Ferguson 1999). This discourages its use for absorption studies. Further, bacterial contamination can also be a problem. Fotos et al (1990) and Williams et al (1994) comment on this, suggesting that they may also influence the $\mathrm{pH}$ of the storage media. For this reason, a number of different disinfectant procedures have been used to block microbial growth in long term salivary immersion studies. Components in the saliva could also form a film on the surface of the specimens, thus, introducing a second semipermeable surface over the resin which would probably affect the water sorption.

The disadvantages of salivary solutions has encouraged the use of different liquid media to simulate oral fluids including various solvents such as ethanol, acetic acid, proprionic acid (Lee et al 1998). The $75 \%$ ethanol/water mixture, recommended by the US Food and Drug Administration as food simulating fluid, has been used by various authors (Ferracane and Condon 1990, Lee et al 1995). Ferracane and Condon (1990), compared the rate of elution from Silux using water and a $75 \%$ ethanol/water mixture and found that $50 \%$ of the leachable components were extracted by water within 3 hours, but $75 \%$ by the 
ethanol/water mixture. However, in many cases it is the time factor to reach equilibrium that is affected and since water is an easy medium to control, it was used as the standard solution in the present experiment.

Different immersing solutions may however affect the leaching of selected components from the resin. When a $75 \%$ ethanol/water solution is used, maximum softening of the resin is obtained since that solution has a solubility parameter which matches that of BisGMA (Wu and McKinney 1982, McKinney and Wu 1985). The solubility parameter describes the ease with which a molecule will penetrate and dissolve within another substance such as a polymer. It has not been clearly established what differences the presence of molecules in the solute makes to the diffusion characteristics but work by Pearson and Braden (1981) suggests that for PMMA the equilibrium uptake of fluid in solutions of glucose is similar to that on immersion in water. This implies that the presence of molecules such as sugars does not affect the equilibrium uptake of the resin, although the rate may vary.

It was considered that the use of salivary substitutes would have complicated the experimental conditions since it would have necessitated their use in the thermo-cycling baths and reservoirs as well. It could be argued that an initial sorption study using water would provide a base line value where conditions are more controlled. Further, many patients immerse their RPDs in water while some may keep them in $100 \%$ humidity having them wrapped in a wet tissue, overnight. For these reasons deionized water was used to provide a comparable and consistent medium from which reproducible results could be obtained. 
Since water uptake and the time taken to reach equilibrium are highly temperature dependent (Vijayaraghavan and Hsiao 1994), the experiment was conducted at a temperature equivalent to that of the oral cavity. Further, the thermo-cycling regime provided a means to simulate, in a controlled manner, the changes in the oral temperatures during eating and drinking. All times that the specimens were not tested, they were kept immersed in water at $37^{\circ} \mathrm{C}$. The only exception to that was during load testing. Since the time of load testing was very short in comparison with the storage time, this was considered to be of minimum significance.

\subsubsection{EFFECTS OF WATER SORPTION UPON THE SPECIMEN}

Cured composite resins are composed of cross-linked molecules. As the resins are water conditioned, water penetrates the matrix and expands the openings between polymer chains. This allows soluble components to diffuse out (Ferracane 1994). Since water sorption is a diffusion process, the dimensions of the specimens for the water sorption test were chosen so that the specimens had a large cross-sectional area. Flat discs of material, ensured that the sorption effects were seen more readily since water can diffuse through both exposed surfaces and permeation was essentially uniform. Normally, discs with very thin cross-section are used. However, in this case to simulate the thickness of the load and thermo-cycled specimens a thickness equal to that of the resin spine of the bonded specimens of the main study was used. This resulted in a more close relationship of the times needed to reach equilibrium between the specimens of the initial water sorption study and the bonded specimens of the main study. On the bonded specimens, water can infiltrate the resin from all sides apart from that which is bonded to metal. The bonded surface of the composite was the one likely to reach equilibrium last as the diffusion pathway is complex. Similarly, the adhesive interface is likely to be the last to reach 
equilibrium completely since water could only reach it through the composite resin spine or through its exposed surface at the side of the specimen.

In the oral environment the time to reach equilibrium could be substantially longer where thick facings are present. During function, the diffusion of water is slowed down when the polymer is in compression, while it is speeded up when the polymer is in tension (Söderholm 1984b). Therefore intraoral loading and the variable dimensions of the facings can frequently lead to variation in the fluid uptake and temporary internal stresses may be set up within the material.

In the water sorption and solubility studies, the specimens were desiccated twice, as initially two processes take place simultaneously. Soluble components were diffusing out of the resin at the same time that water was diffusing in. This produced an apparent water sorption which was less than the real sorption. On the second cycle it was expected that the soluble component would have been eliminated. It was possible to confirm that all soluble material was lost during the first sorption cycle, by establishing the mass at the beginning and end of the second sorption cycle after desiccation were similar. By recording the initial mass at the start of both sorption cycles the amount and mass of the soluble material which has been lost could also be calculated.

\subsubsection{CONTROL OF EXPERIMENTAL VARIATION IN THE WATER SORPTION STUDY}

Since the mass changes are relatively small, a preliminary experiment was carried out to determine variation due to environmental influence. Any mass change that varied more than $\pm 0.0009 \mathrm{~g}$ was regarded as being outside the limits of experimental variation. To ensure a similar weighing procedure all specimens prior to weighing were blot dried with 
a paper towel in a similar manner to remove surface moisture. Although this was carried out with care, it was possible that an inevitable variation in the extent to which the specimens were dried would have been reflected in the amount of surface moisture present on the specimens when weighed. Early weighings are more likely to have been affected by the variation in blotting process, as water may only have been adsorbed onto the surface of the specimens at that time. The effect of this variable decreased with time as the resin became increasingly saturated with water.

\subsubsection{ANALYSIS OF THE EXPERIMENTAL FINDINGS OF THE WATER SORPTION STUDY}

Both Silux Plus and Z100, as seen from the calculated values absorbed most of the water during the first few days (Table 5.11). As the specimens are first immersed in water, there is a tendency for the water to migrate into the polymer, diffusing from a level of higher to one of low concentration, in an attempt to equilibrate the system. This initial, rapid water uptake slows as the equilibrium state is approached. At the same time during the first cycle, as water expands the polymer chains, trapped soluble components and small molecules can diffuse out (Ferracane 1994). After equilibrium is achieved, the desiccation of the specimens removes water which has diffused in. At the end of the first sorption desiccation cycle, the final mass of the samples is less than the starting mass. This difference represents the soluble material which has been lost. Eventually, assuming that equilibrium has been reached, the second cycle will represent sorption only. This may be confirmed by comparing the starting and final mass of the second cycle.

Comparing the corresponding values recorded between the two materials during both cycles, the percentage water uptake is always lower for Z100 compared to Silux Plus (Table 5.11). These findings are not surprising considering the composition of these 
materials. Silux Plus has a higher volume fraction of resin compared to Z100 which is heavily filled limiting the resin matrix between the filler particles, into which water may diffuse. Silux Plus contains prepolymerised organic filler particles and resin matrix, so it is possible that water can diffuse not only in between the filler particles but also within these prepolymerised particles as well. In fact, it is also possible for the pyrolytic silica to be hydrolysed as well. Therefore, due to the difference in the resin content, water sorption has much more of an immediate effect upon the properties of the microfine compared to the hybrid. It would be expected that long-term exposure would result in a greater reduction in the elastic modulus of Silux Plus due to plastisisation.

\subsubsection{SOLUBILITY ESTIMATION}

When the Silux Plus specimens were desiccated a mean mass loss of $0.0163 \mathrm{~g}$ was calculated. The solubility calculated for Silux Plus was $0.61 \%$. When the Z100 specimens were desiccated a mean mass loss of $0.001 \mathrm{~g}$ was calculated. This is only just above the value of experimental variation. The solubility calculated for $\mathrm{Z} 100$ was $0.19 \%$. This is about one third of the solubility calculated for Silux Plus. The greater mass of soluble constituents released from Silux Plus compared to $\mathrm{Z} 100$ reflects the amount and mass of the soluble constituents. Since the origin of soluble components initially is the resin matrix, it is not surprising that more soluble material came out of the Silux Plus. A comparison of the two systems suggests that the variation is due solely to the difference in resin content and there is little evidence of possible filler/resin loss.

A variation in the $\mathrm{pH}$ of the immersion solutions was noted with the $\mathrm{Z100}$ storage solute having a higher $\mathrm{pH}$ with time. This might be attributed to a late stage of hydrolysis of the silane coupling agents on the glass, as proposed by Söderholm (1981), but without further work, this cannot be confirmed. 
These results suggest that Silux Plus is more likely to be influenced by water immersion than Z100 leading to potentially greater plastisisation. However this is partly due to different volumes of resin included in each material. On a volume to volume ratio uptake is very similar.

\subsection{MAIN LOAD CYCLING AND THERMO-CYCLING STUDY}

\subsubsection{SPECIMEN DESIGN}

It is desirable that dental materials should be evaluated using test specimens of clinically appropriate dimensions under conditions that approach, as close as possible, those found clinically. Whether one deals with composite or acrylic resin restorative materials or cast metal alloys, these are all used, often combined together, to fabricate load bearing structures of quite complex shapes, to serve the intraoral needs. The design of in vitro studies to estimate how such complex structures will perform intraorally, is difficult and requires consideration of material's properties and test conditions but also the design and fabrication of test specimens.

In this study, an attempt was made to design specimens which could simulate both a clasp arm and also an occlusal load bearing area. A rectangular specimen consisting of a metal and resin component was designed. Milligan (1953) who worked on cylindrical and rectangular shaped specimens, argued that a rectangular-shaped specimen is generally a more favourable shape for flexural testing. However the results he obtained for the different shape specimens were generally in agreement. In the present study the load was designed to be applied on the middle of the metal part of the specimen. The resin part was never loaded directly or supported directly by the three point bend jig. However, changes within the resin component would reflect on changes of the mechanical properties of the laminate. The dimensions of the metal component allowed mounting in the three point 
bend testing jig for cyclical loading and for a uniaxial three point bend test. The specimen was designed to resemble the resin/metal interface found in denture prostheses and at the same time to allow for easy and reproducible force application.

In the clinical situation where occlusal surfaces are covered with a resin material, the resin component is loaded directly rather than the metal. On the other hand, in our study the metal component was loaded directly instead. However, the load was always applied vertically to the different materials. Therefore, considering the way the stresses are distributed at the interfaces (Figure 6.1 in section 6.5.5.1), the only difference between the two situations at the interfaces is that areas subjected to compression loading become areas of tension and areas of tension become areas of compression. As may be seen below, the effect of this may not be of great significance.

\subsubsection{CONTROL OF EXPERIMENTAL VARIATION DURING SPECIMEN PREPARATION}

In order to standardise the experimental conditions an attempt was made to reduce variation to a minimum. However, the preparation of the specimens presented a number of stages where small variations could have influenced the subsequent results.

\subsubsection{THE CASTINGS}

A number of procedures were adopted to minimise variations in the production of castings with consistent bonding surfaces. A freehand cutting procedure was adopted, during which the wax was cut with the aid of a ruler. Since all dimensions were evaluated visually next to the ruler, small variations of the order of $\pm 0.5 \mathrm{~mm}$ in the size of the specimens were expected. Heat from the warm investment base, could also have caused the pattern to distort. Failure to adequately secure the pattern to the investment base, 
allowed it to lift and this explained some small increase in the casting thickness, estimated to be around $0.2 \mathrm{~mm}$ as measured by a micrometer prior to three point bend testing. This increase in the thickness of some specimens resulted in a variation of the region of $\pm 3.2 \mathrm{GPa}$ in their modulus. This was calculated from tests performed on 10 uncoated metal specimens. To reduce a further variation between metal specimens, the same diameter sprue wax was used for all the castings to ensure that the molten alloy's flow characteristics were not altered during the casting procedure.

The castings were stored together before further preparation and were randomly selected for each treatment. Therefore, any random variation was spread over all groups. To ensure an even preparation of the metal surface by sandblasting, a jig was constructed to ensure a constant distance between the nozzle and specimen surface. However, a freehand technique was adopted, so small variations in the speed of the passage of the jet over the specimens could have resulted in some areas to being abraded more than others. The abraded surfaces of the specimens were assessed with the naked eye to evaluate changes in the grayness of the metal after the surface was blasted. The procedure may have led to variation in topography of the surfaces. This could have influenced the quality and thickness of the oxide layer which developed. Subsequent to air abrasion, all specimens were placed in distilled water and cleaned ultrasonically for 5 minutes to allow for the development of a uniform oxide layer on the alloy surface, and then dried. Subsequently, opaque CESEAD primer and opaque were applied according to the manufacturer's instructions. The thickness of the opaque layer was not measured for individual specimens. A standardised technique was used to deliver similar amounts of the material on the surface of the metal component of the laminate. A similar small amount of opaque was dispensed from the syringe and applied with two single brush stroke movements 
along the length of the specimen. The manufacturer's instructions for application were followed closely during the procedure.

\subsubsection{THE RESINS}

All specimens were initially light polymerised individually inside the specimen mould using a handheld light curing unit. The specimens of each set were then transferred to a light vessel to complete polymerisation. Both units were recently serviced to ensure their polymerising efficiency. Curing was timed with a stop watch. The objective of using two curing cycles was to ensure that the specimens had been exposed to the curing light from all directions and had been adequately cured, since within the mould the specimen sides could not be exposed directly to light. The mould had a transparent cover which covered the surface while polymerisation occurred and the problem of air inhibition of the cure of the resin spines was reduced. It may be assumed that the top surface layer was effectively polymerised after the first curing cycle and that the rate of conversion within the resin was high. Achieving a high rate of conversion should not only reduce the amount of unpolymerised material released on exposure to water but it should also cause a rapid increase in the mechanical properties of the polymer, due to the high level of crosslinking which may be achieved. There is some doubt about the need for secondary curing since the initiation and conversion during the primary phase may have been effective and all cross-linking may have already been established. Nevertheless, the same curing protocol was followed during the water sorption study utilising two curing cycles, in order to ensure that the resin had been equally polymerised in the two experiments.

If care has been taken to avoid the development of an oxygen-inhibition layer, then the surface hardness should reflect the optimum cure attainable in the composites cured (Watts et al 1986 and 1987). On examining the Knoop hardness values of various 
composites after a single curing cycle, they found that the values increased with time. Heavily filled hybrid composites, possibly due to a reduction in segmental mobility, were slower in their increase of hardness values compared to lightly filled ones. The increase was not linear and was more rapid during the first hour and slowing thereafter. In composite resins the relationship between hardness, wear properties and mechanical properties is not clear (Harrison and Draughn 1976).

Eliades et al (1987) and Kildal and Ruyter (1994) have commented on the value that the secondary curing has upon the degree of conversion. The secondary curing inside the light vessel would subject the specimens to all around light exposure together with some heat, so that the conversion at the deeper areas of the specimens should increase compared to the one achieved through curing with the hand held unit only. The improvements in some of the properties of the composites though produced by additional curing may be of only short term benefit since water conditioning alters the resin matrix as the composite equilibrates with water (Ferracane et al 1995).

\subsubsection{MECHANICAL PROPERTIES STUDIED}

In the present study, for each combined metal/resin specimen, the three point bend test recorded the stress at failure and apparent flexural modulus of the bonded specimen. By measuring these two properties an indication of the rigidity of the specimen and also an estimation of the ultimate load which would produce failure could be made. These properties may be associated with the durability of the bonded metal/resin structure in a clinical environment.

Since we were dealing with a composite structure made of resin, adhesive and metal the stress at failure and apparent flexural modulus, reflected the properties of the combined 
structure rather than properties of the individual components. The change of modulus observed between the different groups, is attributed to the laminate construction and the interaction between metal, resin and adhesive components of the specimen.

Generally, cast cobalt-chromium tends to work harden during load cycling. While the modulus of the metal component may have changed, for the purpose of this study we assumed that due to the low cycling load employed, the modulus of the metal within the laminate remained unchanged. A greater contribution to change may have been within the adhesive, due to water sorption with respect to time. Reduction in the adhesive bond strength may lead to failure within the resin component. There is a complex interplay at the interface as the specimen is loaded and this will be discussed in 6.5.5.1.

\subsubsection{THERMO-CYCLING}

Frequently, the clinical performance of restorative materials is simulated by subjecting specimens to thermo-cycling using a variety of temperatures and exposure times. The total thermo-cycling times were chosen, after an estimation of the daily eating behaviour of a small group of subjects, based on an assumption of the times spent in each meal eating hot and cold foods. The duration that the specimens were immersed in the cold and hot water baths was limited to a short immersion time so that the interface between the resin and metal components would be maximally stressed. Six seconds was chosen as a relative short period of time approximating the average time that the food bolus is in contact with the dentition before it gets swallowed.

Thermo-cycling of the specimens through water baths at $60^{\circ} \mathrm{C}, 37^{\circ} \mathrm{C}$ and $4^{\circ} \mathrm{C}$ will generate changes at the resin/metal interface. This is due to the difference in diffusivity values of the composite resin and $\mathrm{Co} / \mathrm{Cr}$ metal alloy component of the specimens. The 
speed that the materials reach an equilibrium temperature will differ substantially, as heat transfer is substantially faster in metal alloys. It is likely, that in the short period of immersion (6 secs), only the $\mathrm{Co} / \mathrm{Cr}$ would have reached the equilibrium temperature of the water bath and since the coefficients of thermal expansion of metals are different from resins any differential change in dimensions of the materials at the interface would weaken the interfacial bond.

\subsubsection{EFFECTS OF THERMO-CYCLING UPON THE SPECIMEN}

External temperature changes are likely to be transmitted rapidly to the interface between metal and adhesive. The heat transmission within the resin is much slower. Both the opaque adhesive resin and the composite veneering resins used during this study, have two components, resin matrix and filler with different coefficients of thermal expansion. It is possible that thermal cycling could create local stresses at the interface between the resin matrix and the filler. The combination of micro-component leakage and accelerated water absorption will degrade this interface and may change the mechanical properties of the resin spine supporting the bonding resin film. Also, it is quite possible that if degradation occurs within the opaque adhesive resin as described by Kern and Thompson (1994) debonding of the resin from the metal might occur. A detailed analysis of the distilled storage water to identify any residue was not undertaken. However, the increased $\mathrm{pH}$ of the long term storage solute (6.98 for Silux Plus and 7.22 for $\mathrm{Z100}$ compared with 6.75 for distilled water) suggests some changes have occurred. This might be associated with changes at the silane coupling interface as suggested by Söderholm (1981).

Vaidyanathan et al (1992) suggested that post curing can occur on heating. Although thermo-cycling may invoke this response, it is considered that this is unlikely to have occurred here, as the two cure system adopted should have achieved almost complete 
conversion initially (Kildal and Ruyter 1994). Temperature change will however accelerate the uptake of water. At the same time the resin may soften since water can act as a plastisiser and lubricant between polymer chains (Takahashi et al 1999).

Hashinger and Fairhurst (1984), Söderholm (1984a), Vaidyanathan et al (1992) and Talib (1993), described the coefficient of thermal expansion of a composite resin decreasing with increasing filler fraction. Söderholm (1984a) found that correlation to be independent of silane treatment. He assumed that the resin forms a weak filler-resin bond even on untreated fillers. The microfine composite has a higher coefficient of thermal expansion $\left(41.6 \times 10^{-6} \% \mathrm{C}\right.$ for Silux Plus) compared to the hybrid composite $\left(22.5 \times 10^{-}\right.$ ${ }^{6} /{ }^{\circ} \mathrm{C}$ for $\mathrm{Z100}$ ) (Versluis et al 1996). The Cesead opaque adhesive resin contains prepolymerised organic filler particles containing colloidal silica similar to Silux Plus, as $10-30 \%$ of its composition. However, Silux Plus contains a higher proportion $40 \%$ by volume, $56 \%$ by weight. The Cesead opaque resin, contains a higher amount of resin matrix compared to that of Silux Plus and its coefficient of thermal expansion is likely to be even higher than that of Silux Plus.

The use of a microfine composite, with a higher coefficient of thermal expansion compared to a hybrid one, will result in potentially greater stress at the interface with the metal. However, if the material is less stiff this may counteract the effect to some degree.

\subsubsection{CONTROL OF EXPERIMENTAL VARIATION DURING THERMO-}

\section{CYCLING}

During thermal cycling there was a temperature fluctuation of around $\pm 1^{\circ} \mathrm{C}$ in the cold water bath $\left(4^{\circ} \mathrm{C}\right)$ and $\pm 1^{\circ} \mathrm{C}$ in the hot water bath $\left(60^{\circ} \mathrm{C}\right)$ after continuous use of around 6 hours. Some water was transferred with the immersion basket from one bath to the other 
causing these temperature fluctuations as the thermocycler cooling and heating elements could not maintain a constant temperature while cycling at the rate used. However, this is unlikely to influence the results since Tanaka et al (1995) found no significant difference in comparing bond strengths after conditioning at $50^{\circ} \mathrm{C}$ vs $60^{\circ} \mathrm{C}$. This suggests that there is a large tolerance in the range of temperatures which may be used to evaluate bond strengths. It seems reasonable to consider that $a \pm 1^{\circ} \mathrm{C}$ difference would have little effect in the current study. Randomisation of specimens under test would also mean that small variation in cycle temperatures should have affected each group the same. In order to minimise this effect, after 6 hours cycling, a 5 minute pause was introduced to allow temperatures to stabilise. During that 5 minute period the specimens were immersed in the $37^{\circ} \mathrm{C}$ bath.

The small size of the water baths of the thermo-cycler precluded the thermo-cycling of all the samples at one time. For logistical reasons, thermo-cycling of single specimens was not practical. Due to the high number of testing groups being thermo-cycled, effective randomisation of the specimens could be achieved. Cycling of a number of specimens together however, may have affected the thermal shock imposed upon individual specimens, the thermal capacity of the total specimens may reduce temperature changes. Also, the location of each specimen within the immersion basket, may affect some variation. However, the small variation in temperature change of the water bath suggests this is not a significant problem.

\subsubsection{LOAD CYCLING}

Generally, assessment of the strength of a metal/resin bond has been based on tensile or shear tests. With published values of tensile bond strength to $\mathrm{Ni} / \mathrm{Cr}$ alloys typically in the region of $40 \mathrm{MPa}$ (Kohli et al 1990, Krueger et al 1990, Aquilino et al 1991) this would 
require loads in the region of 1000 Newtons to debond a retainer with a typical surface area of $25 \mathrm{~mm}^{2}$. Clinically, such loads are unlikely to occur and yet, adhesive or cohesive failure of the resin bond occurs with great regularity (Hussey et al 1991, Creugers et al 1992). It seems likely that current methods of measuring tensile and shear bond strengths do not give a true picture of the failure process. Microcracks may develop within the polymer, when flexurally loaded during continuous intraoral use, and failure can occur when these microcracks join together. The loads required to cause crack initiation and crack propagation within a polymer are considerably lower than might be expected from tensile bond strength measurements (Northeast et al 1994). It is therefore appropriate that flexural strength testing is used as a method of assessing bond strength. Certainly, fatigue stress is likely to play a substantial part in any fracture which occurs intraorally (McCabe et al 1990, Aquilino et al 1991).

The necessary testing equipment was first developed around the 1930's. At the present time, computer controlled testing machines, applying continuous load, have enabled us to carry out flexural loading tests with great accuracy. The most popular tests include the fixed cantilever approach, three point and the four point tests. The choice depends on the nature of the material that is tested. A large displacement at failure may be difficult to accommodate practically. A four point test subjects the beam to a stress distribution which is uniform along it and the peak stress is not concentrated on the loading nose. The three point bend test, induces a localised area of stress upon the tested specimen and therefore may approach more clearly the clinical condition which pertains here (Hargreaves 1983). The three point bend test with the localised area of force application resembles the act of an antagonistic cusp opposing a restoration of the material tested during function. Both in vitro and in vivo situations tend to generate areas of localised stress concentration upon the material tested. The three point bend test also allows an 
adequate extension before fracture, facilitating observation of the mode of failure of the specimen.

The effects of the rate of stressing upon the mechanical behaviour of polymers has been widely investigated. Clinically, chewing rates (58-120 per minute) have been measured for healthy subjects with natural dentitions and no evidence of parafunction (Bates et al 1975). Patients with complete dentures manifest 80-120 chews per minute (Stafford and Smith 1970). The masticatory efficiency of the denture wearing patients, is less than that of dentate subjects. This is attributed to a range of causes which include reduced cutting efficiency of the acrylic teeth, denture movement and reduced occlusal force.

In the present study, for logistical reasons, load cycling at $25 \%$ of the failure load was carried out at an accelerated rate of $5 \mathrm{~Hz}$. This allowed for the specimens to be cycled within a shorter time interval. The failure load was the maximum allowed rate and load level which three consecutive trial specimens had survived the longest cycling time (453,600 cycles). This preliminary trial ensured that the majority of specimens would survive the load cycling, while providing a guide as to the change in the specimen properties which could occur without specimen destruction.

It is suggested that flexural loading on a three point bend test with a variable continuous load applied to the specimen, may simulate the way that biting force is applied intraorally (Hargreaves 1983, Braem et al 1994a). The load imposed on a tooth surface during mastication is not constant (Hu et al 1999). Ahlgren and Öwall (1970) analysed the chewing pattern and found a waveform similar to the sinusoidal waveform of the load applied for fatigue testing. Their results are similar to findings of investigations into the 
pattern and magnitude of occlusal forces by Laurell and Lundgren (1984) and Neill et al (1989).

\subsubsection{EFFECTS OF LOAD CYCLING UPON THE SPECIMEN}

With any flexurally loaded specimens, the surface to which the load is applied, is placed in compression, while the opposing surface is in a state of tension (Figure 6.1). Therefore, the placement of three blocks of material on top of each other, would result in similar compressive and tensional forces for each layer, assuming no friction in between layers. The two underlying layers, the metal part of the laminate and the composite are attached to each other with an adhesive interface. As the load is applied, the underside of one compound of the laminate should be in tension, and the superficial surface of the next in compression. This will lead to a set-up of shear forces at each interface. Cyclical loading will tend to accentuate this effect. Also, it may induce a peeling effect at the interface, since the stresses generated at each interface are antagonistic.

As it can be seen in Figure 6.1 the pattern of stress distribution is complex. Therefore, origin of failure and influence of the various components is difficult to interpret, since it was not possible to study the initiation and propagation of cracks within the specimens.

When a polymer is cyclically loaded, both mechanical and thermal effects can influence its mechanical properties (Beardmore 1979, Hargreaves 1983). Mechanical effects include the viscoelastic response of polymers to load application. Thermal effects include the generation of heat due to hysteresis. This is limited by their low damping capacity; low heat capacity and low thermal diffusivity. Richardson (1989) suggested $30 \mathrm{~Hz}$ as the maximum frequency that effects can be evaluated, before thermal effects predominate. 

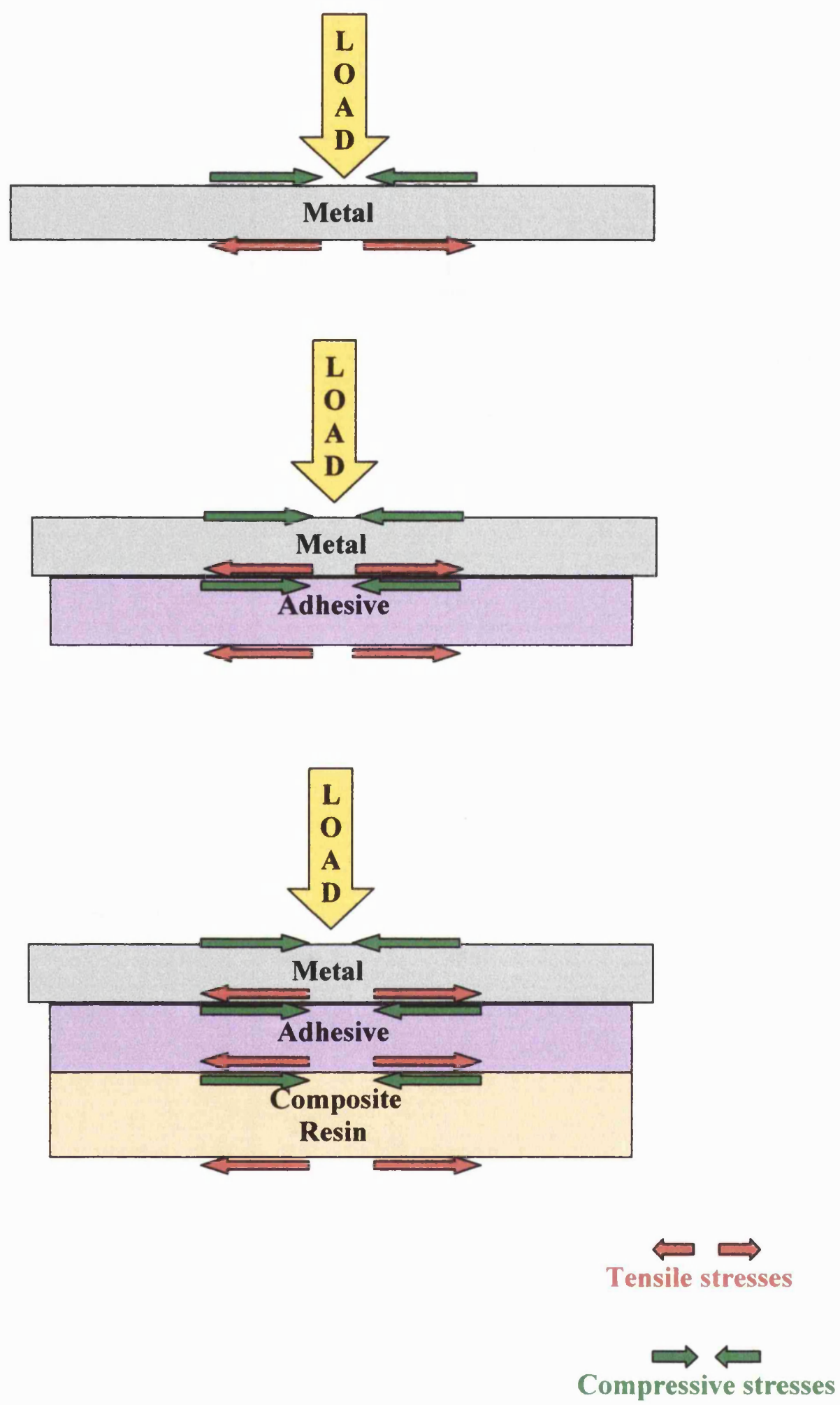

Figure 6.1: Representation of the stresses set-up at the interfaces of the laminate when a load is applied 
For this reason, the selected load cycling frequency of $5 \mathrm{~Hz}$, allowed us to evaluate both the true mechanical effects and thermal effects upon the composites tested.

It has been argued by Llobel et al (1992) that fatigue tests should attempt to use the speed of flexure that materials undergo clinically. Nevertheless, great cycle numbers and large sample sizes require greater cycling frequencies (Wiskott et al 1995). This study was comparative and no attempt has been made to obtain a direct correlation with published clinical values of magnitude of masticatory forces (to predict possible survival rates for the materials tested).

However, the test design with standardised criteria related to clinical findings, permits comparison between the two different composite structures. Although, the level of load necessary to fatigue our specimens could have been worked out using the widely accepted staircase procedure (Draughn 1979, Braem et al 1994b, Braem et al 1995, Yamamoto and Takahashi 1995), it was decided to determine it experimentally. In the staircase method, tests are conducted sequentially, with the maximum applied stress in each succeeding test being increased or decreased by a fixed amount, according to whether the previous stress resulted in a failure or no failure. The staircase procedure has been used to reliably estimate the level of the load, but requires a large number of cycles and sequential specimens tested. Experimental determination of the load was chosen in order to reduce specimens and cycle numbers. The determining factor was not only the level of load used, but also the highest possible frequency that would permit completion of the test within a reasonable time. Initially, $50 \%$ of the load at failure, predetermined from specimens tested to fracture upon manufacture, produced numerous fractures during cycling. The load was then reduced incrementally, to a level at which fractures were not apparent while cycling. All of these trial specimens were examined visually after cycling to detect 
fractures that may have occurred while cycling. Subsequently, the appropriate level of the load applied was determined to be $25 \%$ of the load at failure of the resin. From the results obtained, it appears that the level of load chosen together with the loading frequency did have an appropriate effect on the materials tested.

\subsubsection{CONTROL OF EXPERIMENTAL VARIATION DURING LOAD}

\section{CYCLING}

During each load cycle, a minimum positive load was always applied to each specimen, to avoid impact contact of the crosshead to the metal surface. The extreme sensitivity of the analogue controlled, testing machine ensured that the same positive load was reproduced for each test. The tolerance was set at $0.2-0.8$ Newtons $(<1 \%$ of the immediate failure load). This small variation was substantially smaller than the load at failure values recorded.

\subsubsection{DISCUSSION OF THE EXPERIMENTAL FINDINGS OF THE MAIN LOAD CYCLING AND THERMO-CYCLING STUDY}

Generally, during the first week, the apparent flexural modulus for both Silux Plus and Z100 remained relatively constant (Figures 5.18 and 5.22) whilst the value of stress at failure decreased (Figures 5.26 and 5.30). At this time, the majority of the failures in both test groups were adhesive, between the Cesead opaque resin and the metal surface. This suggests that the bond to the metal surface had not reached its optimum strength. Hansen (1982), Feilzer et al (1990), Schneider et al (1992) and Carvalho et al (1996), have commented on the effect of hydrolytic expansion upon the relief of polymerisation stresses in polymers. Schneider et al (1992) have suggested that thermo-cycling in association with hydrolytic expansion can maintain or even increase the bond strength to metal due to stress relaxation of the polymer, after polymerisation contraction has 
occurred. This will theoretically release stress at the resin metal interface. Although the adhesive interface was not examined after failure, it is possible the bond at the interface became stronger with time. Stress relaxation of the adhesive bonds might have caused the different pattern of failure between week one and the later weeks.

After the first week, there was a tendency for the apparent flexural modulus value to increase for both Silux Plus and Z100 specimens. Silux Plus showed generally, a greater

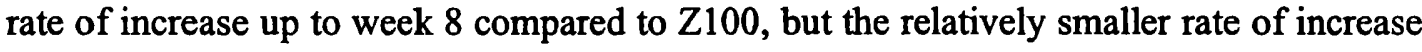
of the latter continued to week 12 .

It is quite possible, as mentioned before, thermo-cycling in association with hydrolytic expansion can maintain or even increase the bond strength to metal due to possible stress relaxation of the polymerisation contraction at the interface (Hansen 1982, Feilzer et al 1990, Schneider et al 1992, Carvalho et al 1996). It can be hypothesised that the increase in the bond strength may also increase the apparent elastic modulus of the laminate since the resin component would be better supported by the metal during testing.

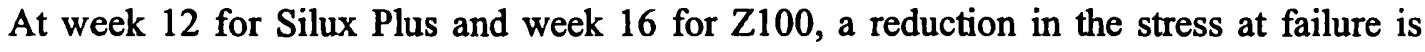
demonstrated, which is similar to the corresponding reduction in the apparent flexural modulus of the same week period. The difference in the amount of resin matrix of the two materials possibly explains the difference in the timing of the observed reduction of the apparent flexural modulus and stress at failure (16 weeks for $\mathrm{Z} 100$ versus 12 weeks for Silux Plus). As mentioned previously, the uptake of water is a diffusion-controlled process, requiring weeks to reach completion. This slow uptake of water correlates with the fact that for the majority of composites, the mechanical properties are only slowly reduced until the polymer matrix is at equilibrium with water (Ferracane et al 1995). 
Z100, a heavily filled composite exhibited low water absorption compared to Silux Plus. Thus, the earlier reduction in mechanical properties of Silux Plus compared to Z100, may be attributed to water uptake and its higher resin component. Water saturation of the resin may have a more immediate effect in Silux Plus.

It is likely that the interface between different prepolymerised filler blocks in Silux Plus is not strong enough to withstand the stresses developed during testing. In all cases the fracture appeared to follow the line of the prepolymerised blocks with some indication of prepolymerised blocks becoming separated from the resin matrix, as the duration of the experiment was extended (Figures $5.35 \mathrm{a}+\mathrm{b}, 5.36 \mathrm{a}+\mathrm{b}$ and $5.37 \mathrm{a}+\mathrm{b}$ ). Since these particles were fully prepolymerised before being incorporated into the polymerisable resin matrix, it is theoretically possible that the chemical interaction of the monomer with the prepolymerised blocks may not be as effective as may theoretically be possible. This may lead to microcrack development at this interface causing these prepolymerised particles to debond. According to Ferracane (1995), there is some indication that the prepolymerised blocks of microfine composites are held together loosely to the polymerising resin matrix due to lack of chemical union.

Z100, due to its high inorganic filler content, and small percentage of resin matrix showed a decrease in its properties much later compared to Silux Plus. Examination of the photomicrographs of the groups conditioned for longer periods indicated that there was some lamination of the material possibly induced by degradation at the resin/filler interface (Figures 5.40a +b). This hypothesis is in agreement with Söderholm (1981 and 1983) who observed a similar degradation of the silane coupling and filler particles as a result of water uptake. An affected silane coupling agent and degraded filler particles would lead to a reduction in the mechanical properties of the material since the cohesive strength of 
the material would be reduced. Crack propagation at the interface will be encouraged by this degradation phenomenon. SEM analysis though, had identified a failure concentrating at the interface between the filler or prepolymerised particles and the resin component.

It is possible, that the thermo-cycling and load cycling equivalent to 24 weeks was enough to increase the temperature within the resin mass, either due to friction on the load cycled groups or due to direct heat application while in the hot water bath. On removal after prolonged load cycling the specimens were hot to touch. The effect of temperature was examined by Draughn (1981) and Kandil et al (1989). They indicated that the increase in temperature may influence the rate of polymerisation of a material with a concomitant increase in its mechanical performance. Again, it is questionable whether this transient temperature rise would influence the material in the light of the extensive curing undertaken during preparation. It is possible though, that unreacted groups could cross-link due to heat either from prolonged load cycling or thermo-cycling. An alternative explanation could be the theory mentioned earlier that water sorption might cause expansion which counteracts any stress built-up at the adhesive interface due to polymerisation contraction as described by Hansen (1982), Feilzer et al (1990), Schneider et al (1992) and Carvalho et al (1996).

It is an interesting finding, that the values for stress at failure for both types of laminates (Z100 and Silux Plus) are comparable. On earlier work by Kountouras et al (1999) using a similar design of specimens and experimental set up but using Cesead as the veneering resin material, the values for stress at failure were also similar. This suggest that the adhesive layer, the only common factor is playing a considerable part in the failure process. 
Both load and thermo-cycling tests might be expected to increase the effects of the water sorption by weakening the matrix either by causing microfractures (load cycling) (Ferracane 1995, Zhao et al 1997) or by stressing the interface in between components with dissimilar coefficients of thermal expansion (thermo-cycling) (Wictorin et al 1989, Soh and Selwyn 1992). There is some evidence that this does occur in the long term groups as the interface between the resin and the filler or prepolymerised particles was affected for all specimens. There was some SEM evidence that load cycling was more effective in debonding the prepolymerised particles or filler material from the resin matrix compared to thermo-cycling. This effect was more marked for Silux Plus. Further work in this area is required to investigate the phenomenon.

\subsubsection{MODE OF FAILURE}

During the dye penetration study, two types of failure were noted for both the materials tested. A cohesive failure, in which the fracture occurred within the resin material, and an adhesive failure in which the fracture occurred at the resin/metal interface. Nevertheless, as mentioned earlier, the origin of failure could not be identified. Therefore, a cohesive failure was registered when the specimen was examined visually and showed an appearance similar to the specimens shown in Figures 5.32a+b and 5.33a+b. On the other hand an adhesive failure was registered when the appearance of the specimen was similar to the one shown in Figures 5.34a+b. Generally, the cracks registered during adhesive failures were longer compared to the cohesive ones (Tables 5.44 and 5.45). Visual examination of the fractured specimens, determined that all fractures occurred in between the Cesead opaque and the $\mathrm{Co} / \mathrm{Cr}$ surface. It seemed that the fracture line was easier to propagate in between the metal surface and the Cesead opaque. From these results it seemed that the bond between the composite veneering resin and the Cesead opaque was always stronger than the bond in between the Cesead opaque and the metal. 
Nevertheless, as described earlier, it is quite possible that failure at the interface could reflect not only bond strength failure but also changes within the resin matrix of the Cesead opaque resin due to hydrolysis. Due to the difference in the nature of metals and polymers, a greater difference in the coefficients of thermal expansion is more likely to be between $\mathrm{Co} / \mathrm{Cr}$ and the Cesead opaque. So in theory this is the area that most of stresses will be set up.

Some specimens that registered a cohesive failure had a fracture line that spread in opposite directions when it reached the metal surface. It is quite possible that differences in the quality of the oxide layer on the metal surface or microscopic flaws of the bonded interface that act as critical stress risers (Toparli and Aksoy 1998) might cause the difference in the way that the crack was propagated. This cannot be confirmed though, since the surface of the castings was only evaluated visually by the operator without any magnification.

Composite resins are not homogeneous materials. Cracks tend to form in areas where there are faults or discontinuities within the material, so there is a possibility that crack propagation would differ between specimens causing the variation mentioned. It is possible also, that the two different types of cohesive failure, reflect different ways in which the deterioration of the adhesive bond or of the opaque resin could have occurred. Although the fractured specimens were not examined, it may be that a double extension might imply a greater deterioration of either the adhesive bond or the opaque resin. Failure could be due to a general deterioration of the area's properties rather than a crack finding itself propagating through a single line of least resistance that does not represent the condition of the local area. 


\subsection{CLINICAL SIGNIFICANCE OF THE RESULTS}

During this study, we observed that the stress at failure for both the materials tested is similar, while the extension at failure tends to be different with the microfine showing a higher value. Since the origin of failure is suggested to be the adhesive interface, the veneering materials are then over-stressed and eventually fail as well.

During testing, two failure modes where observed. A cohesive one, located within the composite veneering resin material and an adhesive one, in between the opaque resin and metal surface. Similarly, failure of adhesive restorations intraorally can either be within the restoration itself due to failure of the material employed (cohesive failure) or failure at the interface where the restoration meets the substrate that it is adhered to (adhesive failure). It can be argued that among the two types of failure, a cohesive one is preferred to an adhesive one. It allows for clinical service to be continued since the fragments are still attached to the substrate without necessitating urgent treatment by the dentist. The mode of failure observations and dye penetration study showed that the crack of an adhesive failure had a greater extension compared to the cracks encountered during a cohesive failure. Generally, the adhesive failure resulted in a greater length of debonding, while a cohesive failure resulted in a smaller fragment line that can occasionally extent in two directions. When a resin composite veneer bonded to a metal substrate fails adhesively, not only intraoral function is compromised since the whole facing could debond but also aesthetics are compromised due to the metal substrate showing. A cohesive failure on the other hand, due to the small extension of the fracture lines allows not only for the clinical function to be continued but also for less discoloration at the interface. 
The elastic properties of the veneering materials tested are different, therefore they can be employed in different intraoral sites depending the requirements. In areas where there are a lot of flexural movements as on a clasp of an RPD, Silux Plus is the preferred material since it allows for similar stress at failure values, a tendency for a higher extension at failure and similar or slightly higher trend for a cohesive type of failure compared to Z100. Z100 exhibited a higher flexural modulus but also a tendency for lower extension at failure, compared to Silux Plus. These facts make Z100 the material of choice in areas where increased rigidity is required and the flexural movements are small as on an onlay occlusal surface of an RPD. Silux Plus being a microfine composite is indicated for areas where appearance is of foremost importance. The present findings suggest that it might require frequent refurbishments due to the higher water sorption and solubility exhibited compared to $\mathrm{Z} 100$ which showed a lower solubility and water sorption value making it potentially a more colour stable material.

The aluminum oxide blasted $\mathrm{Co} / \mathrm{Cr}$ surface has proved to be an appropriate conditioned surface for bonding resin type materials to it. In the tested veneered systems the Cesead primer and opaque are assumed to be the source of failure. No long-term clinical study assessing Cesead's performance bonded to $\mathrm{Co} / \mathrm{Cr}$ has been reported to date. Veneering systems incorporating an adhesive interface with better elastic properties, which could deform to a greater extent, would facilitate support of the veneering resin by the metal substrate, allowing for greater extension at flexure, before failure occurs and this might lead to better clinical performance. 


\section{SUGGESTIONS FOR FURTHER RESEARCH}

The present study suggested that the adhesive resin is the most significant influence on veneer failure. Further clarification of the initiation and pattern of crack propagation would help establish the characteristics of the adhesive resins which allow failure to occur. A number of approaches could be used for this,

firstly, during load cycling:

- Photographic real time monitoring of crack development.

- Non-destructive measurement of the elastic modulus.

- Strain measurement of the different components of the laminate.

- Measurement of temperature gradients to identify "hot spots" by infra red photography.

secondly, during three point bend testing:

- Photographic real-time monitoring of crack propagation at failure.

A further area of research could centre upon more closely simulating the intraoral conditions within which laminate veneers perform. The conditions of testing in the present study could more closely resemble intraoral conditions if artificial saliva had been used and the fatigue testing took place with the specimens immersed in saliva at a controlled temperature.

The duration of the present study attempted to simulate six months usage within the oral cavity. The finding that the flexural modulus of the veneer laminate continues to increase throughout this time period would suggest that even longer term studies are necessary to fully characterise the performance of the laminates. 


\section{CONCLUSIONS}

- The mean flexural modulus of the metal/resin laminates made from the microfine composite was less than that of laminates made from the hybrid composite, following cyclical loading and thermal cycling.

- The flexural moduli of both types of laminate varied with time but showed a significant increase following 24 weeks of conditioning.

- The mean stress at failure of both types of laminates was similar throughout testing.

- The mean extension at failure of the hybrid resin laminates tended to be lower than that of the microfine resin laminates.

- Z100, a hybrid resin, and Silux Plus, a microfine resin, exhibit differences in their water sorption pattern and mechanical properties. Silux Plus has a lower elastic modulus and fails at a lower stress level than Z100. It absorbs more water and shows a higher solubility compared to Z100. 


\section{ACKNOWLEDGEMENTS}

This project could not have been fulfilled without the help, advice and influence of many individuals, to whom I offer my thanks.

Prof. G. J. Pearson and Dr. J. A. Howlett, my supervisors, who provided considerable helpful advice and guidance throughout this study.

Mr. G. Palmer, my laboratory instructor, who assisted and advised with the experimental set-up, during the long load cycling and thermo-cycling periods.

Mr. C. Clark, my instructor technician, who assisted greatly in the production of the castings, without which this study could not have been undertaken. 


\section{REFERENCES}

Abe, Y., Sato, Y., Akagawa, Y., \& Ohkawa, S. 1997, 'An in vitro study of high-strength resin posterior denture tooth wear'. Int J Prosthodont, vol. 10, no. 1, pp. 28-34.

Aboush, Y. E., Mudassir, A., \& Elderton, R. J. 1991, 'Technical note: resin-to-metal bonds mediated by adhesion promoters'. Dent Mater, vol. 7, no. 4, pp. 279-80.

Ahlgren, J., \& Owall, B. 1970, 'Muscular activity and chewing force: a polygraphic study of human mandibular movements'. Arch Oral Biol, vol. 15, no. 4, pp. 271-80.

Antonson, D. E., \& Benedetto, M. D. 1986, 'Ophthalmic concerns when using visible light curing units'. Quintessence Int, vol. 17, no. 10, pp. 679-82.

Anusavice, K. J., \& de Rijk, W. G. 1990, 'Performance of dental biomaterials: conference report'. Dent Mater, vol. 6, no. 1, pp. 69-72.

Aquilino, S. A., Diaz-Arnold, A. M., \& Piotrowski, T. J. 1991, 'Tensile fatigue limits of prosthodontic adhesives'. J Dent Res, vol. 70, no. 3, pp. 208-10.

Asmussen, E. 1975, 'NMR-analysis of monomers in restorative resins'. Acta Odontol Scand, vol. 33, no. 3, pp. 129-34.

Asmussen, E. 1982a, 'Factors affecting the quantity of remaining double bonds in restorative resin polymers'. Scand J Dent Res, vol. 90, no. 6, pp. 490-6.

Asmussen, E. 1982b, 'Restorative resins: hardness and strength vs. quantity of remaining double bonds'. Scand J Dent Res, vol. 90, no. 6, pp. 484-9.

Asmussen, E., \& Peutzfeldt, A. 1990, 'Mechanical properties of heat treated restorative resins for use in the inlay/onlay technique'. Scand J Dent Res, vol. 98, no. 6, pp. 564-7.

Asmussen, E., \& Peutzfeldt, A. 1998, 'Influence of UEDMA BisGMA and TEGDMA on selected mechanical properties of experimental resin composites'. Dent Mater, vol. 14, no. 1, pp. 51-6.

Atmadja, G., \& Bryant, R. W. 1990, 'Some factors influencing the depth of cure of visible light-activated composite resins'. Aust Dent $J$, vol. 35, no. 3, pp. 213-8.

Baharav, H., Brosh, T., Pilo, R., \& Cardash, H. 1997, 'Effect of irradiation time on tensile properties of stiffness and strength of composites'. J Prosthet Dent, vol. 77, no. 5, pp. 471-4.

Bailey, W. J. 1975, 'Cationic polymerisation with expansion in volume'. J Macromol Sci Chem, vol. A9, no. 5, pp. 849-65. 
Bailey, W. J. 1990, 'Matrices that expand on curing for high strength composites'. Mater Sci Eng, vol. A126, pp. 271-9.

Baker, S., Brooks, S. C., \& Walker, D. M. 1988, 'The release of residual monomeric methyl methacrylate from acrylic appliances in the human mouth: an assay for monomer in saliva'. J Dent Res, vol. 67, no. 10, pp. 1295-9.

Barclay, C. W., \& Williams, R. 1994, 'The tensile and shear bond strength of a conventional and a 4-meta self- cure acrylic resin to various surface finishes of $\mathrm{CoCr}$ alloy'. Eur J Prosthodont Restor Dent, vol. 3, no. 1, pp. 5-9.

Barclay, C. W., Spence, D., \& Blunt L. 1997, 'The importance of surface profile and contact surface area on bond strength'. J Dent Res, vol. 76, p. 1021 (Abstract \# 24).

Barkmeier, W. W., Suh, B. I., \& Cooley, R. L. 1991, 'Shear bond strength to dentin and Ni-Cr-Be alloy with the All-Bond universal adhesive system'. J Esthet Dent, vol. 3, no. 4, pp. 148-53.

Barkmeier, W. W., \& Cooley, R. L. 1992, 'Laboratory evaluation of adhesive systems'. Oper Dent, Suppl, no. 5, pp. 50-61.

Barkmeier, W. W., Gendusa, N. J., Thurmond, J. W., \& Triolo, P. T., Jr. 1994, 'Laboratory evaluation of Amalgambond and Amalgambond Plus'. Am J Dent, vol. 7, no. 5, pp. 239-42.

Bates, J. F. 1963, 'Retention of cobalt-chromium partial dentures'. Dent Pract, vol. 14, no. 4, pp. 168-71.

Bates, J. F., Stafford, G. D., \& Harrison, A. 1975, 'Masticatory function--a review of the literature. 1. The form of the masticatory cycle'. J Oral Rehabil, vol. 2, no. 3, pp. 281301.

Bayne, S. C., Taylor, D. F., \& Heymann, H. O. 1992, 'Protection hypothesis for composite wear'. Dent Mater, vol. 8, no. 5, pp. 305-9.

Bayne, S. C., Heymann, H. O., \& Swift, E. J., Jr. 1994, 'Update on dental composite restorations'. J Am Dent Assoc, vol. 125, no. 6, pp. 687-701.

Beardmore, P. 1979, 'Fatigue Behavior of Polymers', in Fatigue Mechanisms, Proceedings of an ASTM-NBS-NSF symposium, ed J. T. Fong, American Society for Testing and Materials, Philadelphia, USA, pp. 453-70.

Beatty, M. W., Swartz, M. L., Moore, B. K., Phillips, R. W., \& Roberts, T. A. 1993, 'Effect of crosslinking agent content, monomer functionality, and repeat unit chemistry on properties of unfilled resins'. J Biomed Mater Res, vol. 27, no. 3, pp. 403-13. 
Berenbaum, R., \& Brodie, I. 1959, 'Measurement of the tensile strength of brittle materials'. Br J Appl Phys, vol. 10, pp. 281-7.

Bertolotti, R. L., Lacy, A. M., \& Watanabe, L. G. 1989, 'Adhesive monomers for porcelain repair'. Int J Prosthodont, vol. 2, no. 5, pp. 483-9.

Bertolotti, R. L., \& Paganetti, C. 1990, 'Adhesion monomers utilized for fixed partial denture (porcelain/metal) repair'. Quintessence Int, vol. 21, no. 7, pp. 579-82.

Boretti, G., Bickel, M., \& Geering, A. H. 1995, 'A review of masticatory ability and efficiency'. J Prosthet Dent, vol. 74, no. 4, pp. 400-3.

Bowen, R. L. 1962, 'Dental filling materials comprising vinyl silane and binder consisting of the reaction product of bis-phenol $\mathrm{A}$ and glysidyl acrylate'. US Patent, no. $3,066,112$.

Bowen, R. L. 1963, 'Properties of a silica reinforced polymer for dental restorations'. $J$ Am Dent Assoc, vol. 66, no. 1, pp. 57-74.

Bowman, A. J., \& Manley, T. R. 1984, 'The elimination of breakages in upper dentures by reinforcement with carbon fibre'. Br Dent $J$, vol. 156, no. 3, pp. 87-9.

Braden, M., Causton, E. E., \& Clarke, R. L. 1976, 'Diffusion of water in composite filling materials'. J Dent Res, vol. 55, no. 5, pp. 730-2.

Braden, M., \& Wright, P. S. 1983, 'Water absorption and water solubility of soft lining materials for acrylic dentures'. J Dent Res, vol. 62, no. 6, pp. 764-8.

Braem, M., Lambrechts, P., Van Doren, V., \& Vanherle, G. 1986a, 'In vivo evaluation of four posterior composites: quantitative wear measurements and clinical behavior'. Dent Mater, vol. 2, no. 3, pp. 106-13.

Braem, M., Lambrechts, P., Van Doren, V., \& Vanherle, G. 1986b, 'The impact of composite structure on its elastic response'. J Dent Res, vol. 65, no. 5, pp. 648-53.

Braem, M., Finger, W., Van Doren, V. E., Lambrechts, P., \& Vanherle, G. 1989, 'Mechanical properties and filler fraction of dental composites'. Dent Mater, vol. 5, no. 5, pp. 346-8.

Braem, M., Lambrechts, P., \& Vanherle, G. 1994a, 'Clinical relevance of laboratory fatigue studies'. J Dent, vol. 22, no. 2, pp. 97-102.

Braem, M. J., Davidson, C. L., Lambrechts, P., \& Vanherle, G. 1994b, 'In vitro flexural fatigue limits of dental composites'. J Biomed Mater Res, vol. 28, no. 12, pp. 1397-402. 
Braem, M. J., Lambrechts, P., Gladys, S., \& Vanherle, G. 1995, 'In vitro fatigue behavior of restorative composites and glass ionomers'. Dent Mater, vol. 11, no. 2, pp. 137-41.

Brantley, C. F., Kanoy, B. E., Jr., \& Sturdevant, J. R. 1986, 'Thermal effects on retention of resin-bonded retainers'. Dent Mater, vol. 2, no. 2, pp. 67-71.

Caeg, C., Leinfelder, K. F., Lacefield, W. R., \& Bell, W. 1990, 'Effectiveness of a method used in bonding resins to metal'. J Prosthet Dent, vol. 64, no. 1, pp. 37-41.

Calais, J. G., \& Söderholm, K. J. 1988, 'Influence of filler type and water exposure on flexural strength of experimental composite resins'. J Dent Res, vol. 67, no. 5, pp. 83640.

Carlos, N. B., \& Harrison, A. 1997, 'The effect of untreated UHMWPE beads on some properties of acrylic resin denture base material'. J Dent, vol. 25, no. 1, pp. 59-64.

Carroll, C. E., \& von Fraunhofer, J. A. 1984, 'Wire reinforcement of acrylic resin prostheses'. J Prosthet Dent, vol. 52, no. 5, pp. 639-41.

Carvalho, R. M., Pereira, J. C., Yoshiyama, M., \& Pashley, D. H. 1996, 'A review of polymerization contraction: the influence of stress development versus stress relief. Oper Dent, vol. 21, no. 1, pp. 17-24.

Caughman, W. F., Rueggeberg, F. A., \& Curtis, J. W., Jr. 1995, 'Clinical guidelines for photocuring restorative resins'. J Am Dent Assoc, vol. 126, no. 9, pp. 1280-2, 1284, 1286.

Cesead Product Information 1997, Kuraray Co., Ltd.,1-12-39, Umeda, Kita-Ku, Osaka 530 , Japan

Chang, J. C., Powers, J. M., \& Hart, D. 1993, 'Bond strength of composite to alloy treated with bonding systems'. J Prosthodont, vol. 2, no. 2, pp. 110-4.

Chung, K. H., \& Hwang, Y. C. 1997, 'Bonding strengths of porcelain repair systems with various surface treatments'. J Prosthet Dent, vol. 78, no. 3, pp. 267-74.

Clark, G. T., \& Carter, M. C. 1985, 'Electromyographic study of human jaw-closing muscle endurance, fatigue and recovery at various isometric force levels'. Arch Oral Biol, vol. 30, no. 7, pp. 563-9.

Coelho, C. M., Rubo, J. H., \& Pegoraro, L. F. 1996, 'Tensile bond strength of a resinous cement to a nickel-chromium alloy modified with five surface treatments'. J Prosthet Dent, vol. 76, no. 3, pp. 246-9.

Combe, E. C., Burke, F. J. T., \& Douglas, W. H. 1999, Dental Biomaterials, Kluwer Academic Publishers, Dordrecht, Netherlands, p.233. 
Condon, J. R., \& Ferracane, J. L. 1998, 'Reduction of composite contraction stress through non-bonded microfiller particles'. Dent Mater, vol. 14, no. 4, pp. 256-60.

Cook, W. D. 1980, 'Factors affecting the depth of cure of UV-polymerized composites'. $J$ Dent Res, vol. 59, no. 5, pp. 800-8.

Covington, J. S., McBride, M. A., Slagle, W. F., \& Disney, A. L. 1985, 'Quantization of nickel and beryllium leakage from base metal casting alloys'. J Prosthet Dent, vol. 54, no. 1, pp. 127-36.

Craig, R. G. 1981, 'Chemistry, composition, and properties of composite resins'. Dent Clin North Am, vol. 25, no. 2, pp. 219-39.

Craig, R. G. 1997a, Restorative dental materials, 10th edn, Mosby-Year Book Inc, St.Louis, USA, p. 409.

Craig, R. G. 1997b, Restorative dental materials, 10th edn, Mosby-Year Book Inc, St.Louis, USA, p. 413.

Craig, R. G. 1997c, Restorative dental materials, 10th edn, Mosby-Year Book Inc, St.Louis, USA, p. 245.

Craig, R. G. 1997d, Restorative dental materials, 10th edn, Mosby-Year Book Inc, St.Louis, USA, p. 255.

Creugers, N. H., Welle, P. R., \& Vrijhoef, M. M. 1988, 'Four bonding systems for resinretained cast metal prostheses'. Dent Mater, vol. 4, no. 2, pp. 85-8.

Creugers, N. H., Kayser, A. F., \& Van't Hof, M. A. 1992, 'A seven-and-a-half-year survival study of resin-bonded bridges'. J Dent Res, vol. 71, no. 11, pp. 1822-5.

Dart, E. C., \& Nemeck, J. 1978, 'Photopolymerisable composition'. US Patent, no. $4,071,424$.

Darvell, B. W. 1990, 'Review: Uniaxial compression tests and the validity of indirect tensile strength'. J Mater Sci, vol. 25, no. 2A, pp. 757-80.

Davidson, C. L., \& Feilzer, A. J. 1997, 'Polymerization shrinkage and polymerization shrinkage stress in polymer- based restoratives'. J Dent, vol. 25, no. 6, pp. 435-40.

Davis, D. M., \& Waters, N. E. 1987, 'An investigation into the fracture behavior of a particulate-filled bis-GMA resin'. J Dent Res, vol. 66, no. 6, pp. 1128-33.

DeBoer, J., Vermilyea, S. G., \& Brady, R. E. 1984, 'The effect of carbon fiber orientation on the fatigue resistance and bending properties of two denture resins'. J Prosthet Dent, vol. 51 , no. 1 , pp. 119-21. 
Diaz-Arnold, A. M., \& Aquilino, S. A. 1989, 'An evaluation of the bond strengths of four organosilane materials in response to thermal stress'. J Prosthet Dent, vol. 62, no. 3, pp. 257-60.

Diaz-Arnold, A. M., Williams, V. D., \& Aquilino, S. A. 1991, 'The effect of film thickness on the tensile bond strength of a prosthodontic adhesive'. J Prosthet Dent, vol. 66 , no. 5, pp. 614-8.

Dixon, D. L., \& Breeding, L. C. 1992, 'The transverse strengths of three denture base resins reinforced with polyethylene fibers'. J Prosthet Dent, vol. 67, no. 3, pp. 417-9.

Dixon, D. L., Breeding, L. C., Hughie, M. L., \& Brown, J. S. 1994, 'Comparison of shear bond strengths of two resin luting systems for a base and a high noble metal alloy bonded to enamel'. J Prosthet Dent, vol. 72, no. 5, pp. 457-61.

Douglas, W. H., Fields, R. P., \& Fundingsland, J. 1989, 'A comparison between the microleakage of direct and indirect composite restorative systems'. J Dent, vol. 17, no. 4, pp. 184-8.

Doukoudakis, A., Cohen, B., \& Tsoutsos, A. 1987, 'A new chemical method for etching metal frameworks of the acid-etched prosthesis'. J Prosthet Dent, vol. 58, no. 4, pp. 421-3.

Dowd, F. J. 1999, 'Saliva and dental caries'. Dent Clin North Am, vol. 43, no. 4, pp. 579 97.

Draughn, R. A. 1979, 'Compressive fatigue limits of composite restorative materials'. $J$ Dent Res, vol. 58, no. 3, pp. 1093-6.

Draughn, R. A. 1981, 'Effects of temperature on mechanical properties of composite dental restorative materials'. J Biomed Mater Res, vol. 15, no. 4, pp. 489-95.

Dunny, J. A., \& King, G. E. 1975, 'Minor connector designs for anterior acrylic resin bases: a preliminary study'. J Prosthet Dent, vol. 34, no. 5, pp. 496-502.

Eick, J. D., Robinson, S. J., Byerley, T. J., \& Chappelow, C. C. 1993a, 'Adhesives and nonshrinking dental resins of the future'. Quintessence Int, vol. 24, no. 9, pp. 632-40.

Eick, J. D., Byerley, T. J., Chappell, R. P., Chen, G. R., Bowles, C. Q., \& Chappelow, C. C. 1993b, 'Properties of expanding SOC/epoxy copolymers for dental use in dental composites'. Dent Mater, vol. 9, no. 2, pp. 123-7.

Ekstrand, K., Ruyter, I. E., \& Wellendorf, H. 1987, 'Carbon/graphite fiber reinforced poly(methyl methacrylate): properties under dry and wet conditions'. J Biomed Mater Res, vol. 21, no. 9, pp. 1065-80. 
Ekstrand, K., \& Ruyter, I. E. 1987, 'Etching patterns of Co-Cr alloys for bonded cast restorations'. J Dent Res, vol. 66, no. 9, pp. 1479-84.

Eliades, G. C., Vougiouklakis, G. J., \& Caputo, A. A. 1987, 'Degree of double bond conversion in light-cured composites'. Dent Mater, vol. 3, no. 1, pp. 19-25.

Eliades, T., Eliades, G., Brantley, W. A., \& Johnston, W. M. 1995, 'Residual monomer leaching from chemically cured and visible light-cured orthodontic adhesives'. Am J Orthod Dentofacial Orthop, vol. 108, no. 3, pp. 316-21.

El-Sherif, M. H., el-Messery, A., \& Halhoul, M. N. 1991, 'The effects of alloy surface treatments and resins on the retention of resin-bonded retainers'. J Prosthet Dent, vol. 65 , no. 6, pp. 782-6.

Feilzer, A. J., de Gee, A. J., \& Davidson, C. L. 1990, 'Relaxation of polymerization contraction shear stress by hygroscopic expansion'. J Dent Res, vol. 69, no. 1, pp. 36-9.

Feilzer, A. J., de Gee, A. J., \& Davidson, C. L. 1993, 'Setting stresses in composites for two different curing modes'. Dent Mater, vol. 9, no. 1, pp. 2-5.

Ferguson, D. B. 1999, 'The flow rate and composition of human labial gland saliva'. Arch Oral Biol, vol. 44 Suppl 1, pp. S11-4.

Ferracane, J. L., \& Condon, J. R. 1990, 'Rate of elution of leachable components from composite'. Dent Mater, vol. 6, no. 4, pp. 282-7.

Ferracane, J. L. 1994, 'Elution of leachable components from composites'. J Oral Rehabil, vol. 21, no. 4, pp. 441-52.

Ferracane, J. L. 1995, 'Current trends in dental composites'. Crit Rev Oral Biol Med, vol. 6 , no. 4, pp. 302-18.

Ferracane, J. L., Hopkin, J. K., \& Condon, J. R. 1995, 'Properties of heat-treated composites after aging in water'. Dent Mater, vol. 11, no. 6, pp. 354-8.

Ferrari, M., Cagidiaco, M. C., \& Breschi, R. 1987, 'Microscopic examination of resin bond to enamel and retainer with a phosphate monomer resin'. J Prosthet Dent, vol. 57, no. 3, pp. 298-301.

Finger, W. J. 1988, 'Dentin bonding agents. Relevance of in vitro investigations'. Am J Dent, vol. 1, no. Spec No, pp. 184-8.

Fløystrand, F., Kleven, E., \& Øilo, G. 1982, 'A novel miniature bite force recorder and its clinical application'. Acta Odontol Scand, vol. 40, no. 4, pp. 209-14. 
Fotos, P. G., Diaz-Arnold, A. M., \& Williams, V. D. 1990, 'Effect of microbial contamination and $\mathrm{pH}$ changes in storage solutions during in vitro assays of bonding agents'. Dent Mater, vol. 6, no. 3, pp. 154-7.

Fowler, C. S., Swartz, M. L., Moore, B. K., \& Rhodes, B. F. 1992, 'Influence of selected variables on adhesion testing'. Dent Mater, vol. 8, no. 4, pp. 265-9.

Freilich, M. A., Goldberg, A. J., Gilpatrick, R. O., \& Simonsen, R. J. 1992, 'Direct and indirect evaluation of posterior composite restorations at three years'. Dent Mater, vol. 8 , no. 1 , pp. $60-4$.

Gale, M. S., \& Darvell, B. W. 1999, 'Thermal cycling procedures for laboratory testing of dental restorations'. $J$ Dent, vol. 27 , no. 2, pp. 89-99.

Garcia-Godoy, F., Kaiser, D. A., Malone, W. F., \& Hubbard, G. 1991, 'Shear bond strength of two resin adhesives for acid-etched metal prostheses'. J Prosthet Dent, vol. 65 , no. 6, pp. 787-9.

Gates, W. D., Diaz-Arnold, A. M., Aquilino, S. A., \& Ryther, J. S. 1993, 'Comparison of the adhesive strength of a BIS-GMA cement to tin-plated and non-tin-plated alloys'. $J$ Prosthet Dent, vol. 69, no. 1, pp. 12-6.

Gilbert, J. L., Ney, D. S., \& Lautenschlager, E. P. 1995, 'Self-reinforced composite poly(methyl methacrylate): static and fatigue properties'. Biomaterials, vol. 16, no. 14, pp. 1043-55.

Griffith, A. A. 1920, 'The phenomenon of rupture and flow in solids'. Phil Trans Series, vol. 221, pp. 163-98.

Gutteridge, D. L. 1988, 'The effect of including ultra-high-modulus polyethylene fibre on the impact strength of acrylic resin'. Br Dent J, vol. 164, no. 6, pp. 177-80.

Gutteridge, D. L. 1992, 'Reinforcement of poly(methyl methacrylate) with ultra-highmodulus polyethylene fibre'. $J$ Dent, vol. 20 , no. 1 , pp. 50-4.

Guzman, H. J., Swartz, M. L., \& Phillips, R. W. 1969, 'Marginal leakage of dental restorations subjected to thermal stress'. J Prosthet Dent, vol. 21, no. 2, pp. 166-75.

Hacker, C. H., Wagner, W. C., \& Razzoog, M. E. 1996, 'An in vitro investigation of the wear of enamel on porcelain and gold in saliva'. J Prosthet Dent, vol. 75, no. 1, pp. 147.

Hansen, E. K. 1982, 'Visible light-cured composite resins: polymerization contraction, contraction pattern and hygroscopic expansion'. Scand J Dent Res, vol. 90, no. 4, pp. 329-35. 
Hanson, G. H., Gibbon, W. M., \& Shimizu, H. 1983, 'Bonding bases coated with porous metal powder: a comparison with foil mesh'. Am J Orthod, vol. 83, no. 1, pp. 1-4.

Hansson, O. 1990, 'Strength of bond with Comspan Opaque to three silicoated alloys and titanium'. Scand J Dent Res, vol. 98, no. 3, pp. 248-56.

Hansson, O., \& Moberg, L. E. 1993, 'Evaluation of three silicoating methods for resinbonded prostheses'. Scand J Dent Res, vol. 101, no. 4, pp. 243-51.

Hargreaves, A. S. 1983, 'The effects of cyclic stress on dental polymethylmethacrylate. II. Flexural fatigue'. J Oral Rehabil, vol. 10, no. 2, pp. 137-51.

Harrison, A., \& Draughn, R. A. 1976, 'Abrasive wear, tensile strength, and hardness of dental composite resins--is there a relationship?'. J Prosthet Dent, vol. 36, no. 4, pp. 395-8.

Harrison, A., Huggett, R., \& Jagger, R. C. 1978, 'The effect of a cross-linking agent on the abrasion resistance and impact strength of an acrylic resin denture base material'. $J$ Dent, vol. 6, no. 4, pp. 299-304.

Hashinger, D. T., \& Fairhurst, C. W. 1984, 'Thermal expansion and filler content of composite resins'. J Prosthet Dent, vol. 52, no. 4, pp. 506-10.

Hellsing, G. 1980, 'On the regulation of interincisor bite force in man'. J Oral Rehabil, vol. 7 , no. 5 , pp. 403-11.

Hobkirk, J. A., Goodman, J. R., \& Reynolds, I. R. 1989, 'Component failure in removable partial dentures for patients with severe hypodontia'. Int J Prosthodont, vol. 2, no. 4, pp. 327-30.

Hofstede, T., McConnell, R. J., Gratton, D. R., \& Quinn, F. 1990, 'Bond strengths of Panavia-X to treated metal surfaces'. J Dent Res, vol. 69, p. 172 (Abstract \# 508).

Hu, X., Harrington, E., Marquis, P. M., \& Shortall, A. C. 1999, 'The influence of cyclic loading on the wear of a dental composite'. Biomaterials, vol. 20, no. 10, pp. 907-12.

Huang, G. T., \& Söderholm, K. J. 1989, 'In vitro investigation of shear bond strength of a phosphate based dentinal bonding agent'. Scand J Dent Res, vol. 97, no. 1, pp. 84-92.

Hussey, D. L., Gratton, D. R., McConnell, R. J., \& Sands, T. D. 1989, 'The quality of bonded retainers from commercial dental laboratories'. J Dent Res, vol. 68, p. 919 (Abstract \# 424)

Hussey, D. L., Pagni, C., \& Linden, G. J. 1991, 'Performance of 400 adhesive bridges fitted in a restorative dentistry department'. $J$ Dent, vol. 19, no. 4, pp. 221-5. 
Imbery, T. A., \& Davis, R. D. 1993, 'Evaluation of tin plating systems for a high-noble alloy'. Int J Prosthodont, vol. 6, no. 1, pp. 55-60.

Indrani, D. J., Cook, W. D., Televantos, F., Tyas, M. J., \& Harcourt, J. K. 1995, 'Fracture toughness of water-aged resin composite restorative materials'. Dent Mater, vol. 11, no. 3, pp. 201-7.

Irwin, G. R. 1957, 'Analysis of stresses and strains near the end of a crack transversing a plate'. J Appl Mech, vol. 24, pp. 361-4.

Ishijima, T., Caputo, A. A., \& Mito, R. 1992, 'Adhesion of resin to casting alloys'. $J$ Prosthet Dent, vol. 67, no. 4, pp. 445-9.

Jagger, D. C., \& Harrison, A. 1994, 'An in vitro investigation into the wear effects of unglazed, glazed, and polished porcelain on human enamel'. J Prosthet Dent, vol. 72, no. 3 , pp. 320-3.

Jagger, D. C., \& Harrison, A. 1995, 'An in vitro investigation into the wear effects of selected restorative materials on enamel'. J Oral Rehabil, vol. 22, no. 4, pp. 275-81.

Jagger, D. C., \& Harrison, A. 1999, 'The effect of chopped poly(methyl methacrylate) fibers on some properties of acrylic resin denture base material'. Int J Prosthodont, vol. 12 , no. 6 , pp. 542-6.

Jagger, D. C., Harrison, A., \& Jandt, K. D. 1999, 'The reinforcement of dentures'. J Oral Rehabil, vol. 26, no. 3, pp. 185-94.

Jenkins, G. N. 1981, 'A century of oral physiology'. Br Dent J, vol. 151, no. 1, pp. 6-10.

Jones, T. K., Hansen, C. A., Singer, M. T., \& Kessler, H. P. 1986, 'Dental implications of nickel hypersensitivity'. J Prosthet Dent, vol. 56, no. 4, pp. 507-9.

Jones, D. W., \& Rizkalla, A. S. 1996, 'Characterization of experimental composite biomaterials'. J Biomed Mater Res, vol. 33, no. 2, pp. 89-100.

Jørgensen, K. D., \& Asmussen, E. 1978, 'Occlusal abrasion of a composite restorative resin with ultra-fine filler--an initial study'. Quintessence Int, vol. 9, no. 6, pp. 73-8.

Kanca, J. d. 1991, 'Dental adhesion and the All-Bond system'. J Esthet Dent, vol. 3, no. 4, pp. 129-32.

Kandil, S. H., Kamar, A. A., Shaaban, S. A., Taymour, N. M., \& Morsi, S. E. 1989, 'Effect of temperature and ageing on the mechanical properties of dental polymeric composite materials'. Biomaterials, vol. 10, no. 8, pp. 540-4. 
Kanie, T., Fujii, K., Arikawa, H., \& Inoue, K. 2000, 'Flexural properties and impact strength of denture base polymer reinforced with woven glass fibers'. Dent Mater, vol. 16 , no. 2 , pp. $150-8$.

Kawai, K., \& Leinfelder, K. F. 1995, 'In vitro evaluation of OCA wear resistance of posterior composites'. Dent Mater, vol. 11, no. 4, pp. 246-51.

Kern, M., \& Thompson, V. P. 1993a, 'Sandblasting and silica-coating of dental alloys: volume loss, morphology and changes in the surface composition'. Dent Mater, vol. 9, no. 3, pp. 151-61.

Kern, M., \& Thompson, V. P. 1993b, 'Tensile bond strength of new adhesive systems to In-Ceram ceramic'. J Dent Res, vol. 72, p. 369 (Abstract \# 2124).

Kern, M., \& Thompson, V. P. 1994, 'Influence of prolonged thermal cycling and water storage on the tensile bond strength of composite to NiCr alloy'. Dent Mater, vol. 10, no. 1 , pp. $19-25$.

Kern, M., \& Thompson, V. P. 1995, 'Durability of resin bonds to a cobalt-chromium alloy'. J Dent, vol. 23, no. 1, pp. 47-54.

Khan, Z., von Fraunhofer, J. A., \& Razavi, R. 1987, 'The staining characteristics, transverse strength, and microhardness of a visible light-cured denture base material'. $J$ Prosthet Dent, vol. 57, no. 3, pp. 384-6.

Kildal, K. K., \& Ruyter, I. E. 1994, 'How different curing methods affect the degree of conversion of resin- based inlay/onlay materials'. Acta Odontol Scand, vol. 52, no. 5, pp. 315-22.

Kim, K. H., Park, J. H., Imai, Y., \& Kishi, T. 1994, 'Microfracture mechanisms of dental resin composites containing spherically-shaped filler particles'. J Dent Res, vol. 73, no. 2, pp. 499-504.

Knudsen, M. B., \& Jørgensen, K. D. 1983, 'Abrasion of microfill restorative resins in Class 1 cavities'. Scand J Dent Res, vol. 91, no. 2, pp. 159-61.

Koczorowski, R., \& Wloch, S. 1999, 'Evaluation of wear of selected prosthetic materials in contact with enamel and dentin'. J Prosthet Dent, vol. 81, no. 4, pp. 453-9.

Kohli, S., Levine, W. A., Grisius, R. J., \& Fenster, R. K. 1990, 'The effect of three different surface treatments on the tensile strength of the resin bond to nickelchromium-beryllium alloy'. J Prosthet Dent, vol. 63, no. 1, pp. 4-8.

Kountouras, C. G., Howlett, J. A., \& Pearson, G. J. 1999, 'Flexural and thermal cycling of resins for veneering removable overlay dentures'. J Dent, vol. 27, no. 5, pp. 367-72. 
Kourtis, S. G. 1997, 'Bond strengths of resin-to-metal bonding systems'. J Prosthet Dent, vol. 78 , no. 2, pp. 136-45.

Krejci, I., \& Lutz, F. 1991, 'Marginal adaptation of Class V restorations using different restorative techniques'. J Dent, vol. 19, no. 1, pp. 24-32.

Krueger, G. E., Diaz-Arnold, A. M., Aquilino, S. A., \& Scandrett, F. R. 1990, 'A comparison of electrolytic and chemical etch systems on the resin-to- metal tensile bond strength'. J Prosthet Dent, vol. 64, no. 5, pp. 610-7.

Kubo, S., Finger, W. J., Muller, M., \& Podszun, W. 1991, Principles and mechanisms of bonding with dentin adhesive materials'. $J$ Esthet Dent, vol. 3, no. 2, pp. 62-9.

Kulzer \& Co. Gmbh. 1938, 'Improvements in or relating to the manufacture of surgical prostheses'. UK Patent, no. 484,343.

Ladizesky, N. H., Chow, T. W., \& Ward, I. M. 1990, 'The effect of highly drawn polyethylene fibres on the mechanical properties of denture base resins'. Clin Mater, vol. 6 , no. 3, pp. 209-25.

Ladizesky, N. H., Ho, C. F., \& Chow, T. W. 1992, 'Reinforcement of complete denture bases with continuous high performance polyethylene fibers'. J Prosthet Dent, vol. 68, no. 6 , pp. 934-9.

Ladizesky, N. H., \& Chow, T. W. 1992, 'The effect of interface adhesion, water immersion and anatomical notches on the mechanical properties of denture base resins reinforced with continuous high performance polyethylene fibres'. Aust Dent $J$, vol. 37, no. 4 , pp. $277-89$.

Ladizesky, N. H., Cheng, Y. Y., Chow, T. W., \& Ward, I. M. 1993, 'Acrylic resin reinforced with chopped high performance polyethylene fiber--properties and denture construction'. Dent Mater, vol. 9, no. 2, pp. 128-35.

Lammie, G. A., \& Laird, W. R. 1986, in Osborne and Lammie's partial dentures. $5^{\text {th }}$ Ed., London, Blackwell Scientific Publications, Chapter 1, p.5.

Lambrechts, P., \& Vanherle, G. 1983, 'Structural evidences of the microfilled composites'. J Biomed Mater Res, vol. 17, no. 2, pp. 249-60.

Laufer, B. Z., Nicholls, J. I., \& Townsend, J. D. 1988, 'SiOx-C coating: a composite-tometal bonding mechanism'. J Prosthet Dent, vol. 60, no. 3, pp. 320-7.

Laurell, L., \& Lundgren, D. 1984, 'A standardized programme for studying the occlusal force pattern during chewing and biting in prosthetically restored dentitions'. J Oral Rehabil, vol. 11, no. 1, pp. 39-44. 
Lee, S. Y., Greener, E. H., \& Menis, D. L. 1995, 'Detection of leached moieties from dental composites in fluids simulating food and saliva'. Dent Mater, vol. 11, no. 6, pp. 348-53.

Lee, S. Y., Huang, H. M., Lin, C. Y., \& Shih, Y. H. 1998, 'Leached components from dental composites in oral simulating fluids and the resultant composite strengths'. $J$ Oral Rehabil, vol. 25, no. 8, pp. 575-88.

Leinfelder, K. F. 1985, 'Composite resins'. Dent Clin North Am, vol. 29, no. 2, pp. 35971.

Leinfelder, K. F. 1993, 'Composite resin systems for posterior restorations'. Caulk restorative supplement, April, pp.23-27.

Leinfelder, K. F. 1994, 'Resin to metal bonding: overcoming esthetic problems'. J Am Dent Assoc, vol. 125, no. 3, pp. 292-4.

Leinfelder, K. F. 1995, 'Posterior composite resins: the materials and their clinical performance'. J Am Dent Assoc, vol. 126, no. 5, pp. 663-4, 667-8, 671-2 passim.

Leinfelder, K. F., Wilder, A. D., Jr., \& Teixeira, L. C. 1986, 'Wear rates of posterior composite resins'. J Am Dent Assoc, vol. 112, no. 6, pp. 829-33.

Livaditis, G. J. 1986, 'A chemical etching system for creating micromechanical retention in resin-bonded retainers'. J Prosthet Dent, vol. 56, no. 2, pp. 181-8.

Livaditis, G. J., \& Thompson, V. P. 1982, 'Etched castings: an improved retentive mechanism for resin-bonded retainers'. J Prosthet Dent, vol. 47, no. 1, pp. 52-8.

Llobell, A., Nicholls, J. I., Kois, J. C., \& Daly, C. H. 1992, 'Fatigue life of porcelain repair systems'. Int J Prosthodont, vol. 5, no. 3, pp. 205-13.

Lloyd, C. H., \& Iannetta, R. V. 1982, 'The fracture toughness of dental composites. I. The development of strength and fracture toughness'. J Oral Rehabil, vol. 9, no. 1, pp. 5566.

Lösche, G. M., Schürg, C., \& Roulet, J. F. 1993, 'The influence of curing light intensity on marginal adaptation of composite fillings'. J Dent Res, vol. 72(special issue), p.264 (Abstract \# 1284).

Love, L. D., \& Breitman, J. B. 1985, 'Resin retention by immersion-etched alloy'. $J$ Prosthet Dent, vol. 53, no. 5, pp. 623-4.

Lüthy, H., Marinello, C. P., \& Schärer, P. 1990, 'Factors influencing metal-resin tensile bond strength to filled composites'. Dent Mater, vol. 6, no. 2, pp. 73-7. 
Lutz, F., \& Phillips, R. W. 1983, 'A classification and evaluation of composite resin systems'. J Prosthet Dent, vol. 50, no. 4, pp. 480-8.

Mahalick, J. A., Knap, F. J., \& Weiter, E. J. 1971, 'Occusal wear in prosthodontics'. J Am Dent Assoc, vol. 82, no. 1, pp. 154-9.

Mair, L. H. 1995, 'Wear patterns in two amalgams and three posterior composites after 5 years' clinical service'. J Dent, vol. 23, no. 2, pp. 107-12.

Mandel, I. D. 1974, 'Relation of saliva and plaque to caries'. J Dent Res, vol. 53, no. 2, pp. 246-66.

Manley, T. R., Bowman, A. J., \& Cook, M. 1979, 'Denture bases reinforced with carbon fibres'. Br Dent $J$, vol. 146, no. 1, pp. 25.

Mante, F., Saleh, N., \& Mante, M. 1993, 'Softening patterns of post-cure heat-treated dental composites'. Dent Mater, vol. 9, no. 5, pp. 325-31.

Matsumura, H., Varga, J., \& Masuhara, E. 1986, 'Composite type adhesive opaque resin'. Dent Mater J, vol. 5, no. 1, pp. 83-90.

Matsumura, H., Kawahara, M., Tanaka, T., \& Atsuta, M. 1991, 'Surface preparations for metal frameworks of composite resin veneered prostheses made with an adhesive opaque resin'. J Prosthet Dent, vol. 66, no. 1, pp. 10-5.

Matsumura, H., Tanaka, T., Taira, Y., \& Atsuta, M. 1996, 'Bonding of a cobalt-chromium alloy with acidic primers and tri-n- butylborane-initiated luting agents'. J Prosthet Dent, vol. 76 , no. 2, pp. 194-9.

Mazer, R. B., \& Leinfelder, K. F. 1992, 'Evaluating a microfill posterior composite resin. A five-year study'. J Am Dent Assoc, vol. 123, no. 4, pp. 32-8.

McCabe, J. F., \& Basker, R. M. 1976, 'Tissue sensitivity to acrylic resin. A method of measuring the residual monomer content and its clinical application'. Br Dent J, vol. 140 , no. 10 , pp. $347-50$.

McCabe, J. F., Carrick, T. E., Chadwick, R. G., \& Walls, A. W. 1990, 'Alternative approaches to evaluating the fatigue characteristics of materials'. Dent Mater, vol. 6, no. 1 , pp. 24-8.

McCabe, J. F., \& Kagi, S. 1991, 'Mechanical properties of a composite inlay material following post- curing'. $B r$ Dent $J$, vol. 171 , no. 8, pp. 246-8.

McCaughey, A. D. 1993, 'Sandblasting and tin-plating-surface treatments to improve bonding with resin cements'. Dent Update, vol. 20, no. 4, pp. 153-7. 
McConnell, R. J., Taylor, D., \& Moriarity, K. 1989, 'Bonding strengths of resins to noble and base metal alloys'. J Dent Res, vol. 68, p. 955 (Abstract \# 711).

McKinney, J. E., \& Wu, W. 1985, 'Chemical softening and wear of dental composites'. $J$ Dent Res, vol. 64, no. 11, pp. 1326-31.

Miettinen, V. M., \& Vallittu, P. K. 1997, 'Release of residual methyl methacrylate into water from glass fibre- poly(methyl methacrylate) composite used in dentures'. Biomaterials, vol. 18, no. 2, pp. 181-5.

Milligan, L. H. 1953, 'Note on modulus of rupture of cylindrical ceramic rods when tested on a short span'. J Am Ceram Soc, vol. 36, no. 5, pp. 159-161.

Moffa, J. P., Guckes, A. D., Okawa, M. T., \& Lilly, G. E. 1973, 'An evaluation of nonprecious alloys for use with porcelain veneers. Part II. Industrial safety and biocompatibility'. J Prosthet Dent, vol. 30, no. 4, pp. 432-41.

Mohsen, N. M., \& Craig, R. G. 1995a, 'Effect of silanation of fillers on their dispersability by monomer systems'. J Oral Rehabil, vol. 22, no. 3, pp. 183-9.

Mohsen, N. M., \& Craig, R. G. 1995b, 'Hydrolytic stability of silanated zirconia-silicaurethane dimethacrylate composites'. J Oral Rehabil, vol. 22, no. 3, pp. 213-20.

Momoi, Y., Iwase, H., Nakano, Y., Kohno, A., Asanuma, A., \& Yanagisawa, K. 1990, 'Gradual increases in marginal leakage of resin composite restorations with thermal stress'. J Dent Res, vol. 69, no. 10, pp. 1659-63.

Montes, G. G., \& Draughn, R. A. 1987, 'Slow crack propagation in composite restorative materials'. J Biomed Mater Res, vol. 21, no. 5, pp. 629-42.

Moulin, P., Picard, B., \& Degrange, M. 1999, 'Water resistance of resin-bonded joints with time related to alloy surface treatment'. J Dent, vol. 27 , no. 1, pp. 79-87.

Neill, D. J., Kydd, W. L., Nairn, R. I., \& Wilson, J. 1989, 'Functional loading of the dentition during mastication'. J Prosthet Dent, vol. 62, no. 2, pp. 218-28.

Nergiz, I., Pfeiffer, P., \& Niedermeier, W. 1997, 'Effects of surface treatment on resin veneer/alloy bond strength'. J Dent Res, vol. 76, p. 312 (Abstract \# 2390).

Nicholls, J. I., \& Shue, S. L. 1986, 'Effect of bead spacing on the tensile bond strength of resin veneers to cast alloys'. Quintessence Dent Technol, vol. 10, no. 8, pp. 511-5.

Nishiyama, N., Ishizaki, T., Horie, K., Tomari, M., \& Someya, M. 1991, 'Novel polyfunctional silanes for improved hydrolytic stability at the polymer-silica interface'. J Biomed Mater Res, vol. 25, no. 2, pp. 213-21. 
Nohrström, T. J., Vallittu, P. K., \& Yli-Urpo, A. 2000, 'The effect of placement and quantity of glass fibers on the fracture resistance of interim fixed partial dentures'. Int $J$ Prosthodont, vol. 13, no. 1, pp. 72-8.

Northeast, S. E., van Noort, R., \& Shaglouf, A. S. 1994, 'Tensile peel failure of resinbonded $\mathrm{Ni} / \mathrm{Cr}$ beams: an experimental and finite element study'. $J$ Dent, vol. 22, no. 4, pp. 252-6.

Nutt, M. C.1976, 'Metal failure analysis' in Metallurgy and plastics for engineers, Pergamon Press Ltd, Oxford, England, pp.359-83.

O'Brien, W. J. 1997, Dental materials and their selection, 2nd edn, Quintessence Publishing Co Inc, Illinois, USA, p. 393.

Ogle, R. E., David, L. J., \& Ortman, H. R. 1985, 'Clinical wear study of a new tooth material: Part II'. J Prosthet Dent, vol. 54, no. 1, pp. 67-75.

Ohno, H., Araki, Y., \& Sagara, M. 1986, 'The adhesion mechanism of dental adhesive resin to the alloy-- relationship between $\mathrm{Co}-\mathrm{Cr}$ alloy surface structure analyzed by ESCA and bonding strength of adhesive resin'. Dent Mater J, vol. 5, no. 1, pp. 46-65.

Ohno, H., Araki, Y., \& Endo, K. 1992, 'A new method for promoting adhesion between precious metal alloys and dental adhesives'. J Dent Res, vol. 71, no. 6, pp. 1326-31.

Omura, I., Yamauchi, J., Harada, I., \& Wada, T. 1984, 'Adhesive and mechanical properties of a new dental adhesive'. J Dent Res, vol. 63, p. 233 (Abstract \# 561).

Orchard, N. A., Howlett, J. A., Davies, E. H., \& Pearson, G. J. 1997, Adhesive composite resins for artificial teeth: a laboratory investigation of bond strength to a cobaltchromium alloy'. Biomaterials, vol. 18, no. 13, pp. 935-8.

Osborne, J. \& Lammie, G. A. 1953, 'Some observations concerning chrome cobalt denture bases'. Br Dent $J$, vol. 94, no. 3, pp. 55-67.

Özcan, M., Pfeiffer, P., \& Nergiz, I. 1998, 'A brief history and current status of metal-and ceramic surface- conditioning concepts for resin bonding in dentistry'. Quintessence Int, vol. 29 , no. 11, pp. 713-24.

Paffenbarger, G. C., Nelsen, R. J., \& Sweeney, W. T. 1953, 'Direct and indirect filling resins: a review of some physical and chemical properties'. J Am Dent Assoc, vol. 47, no. 5, pp. 516-24.

Pagniano, R. P., \& Johnston, W. M. 1996, 'Three-year effect of unfilled resin dilution on water sorption of a light-cured microfill and hybrid composite resin'. J Prosthet Dent, vol. 75 , no. 4 , pp. 364-6. 
Papadogianis, Y., Boyer, D. B., \& Lakes, R. S. 1985, 'Creep of posterior dental composites'. J Biomed Mater Res, vol. 19, no. 1, pp. 85-95.

Paphangkorakit, J., \& Osborn, J. W. 1998, 'Effects on human maximum bite force of biting on a softer or harder object'. Arch Oral Biol, vol. 43, no. 11, pp. 833-9.

Pearson, G. J. 1979, 'Long term water sorption and solubility of composite filling materials'. J Dent, vol. 7, no. 1, pp. 64-8.

Pearson, G. J., \& Braden, M. 1981. 'Sorption of water by P.M.M.A. from aqueous solutions'. J Dent Res, vol. 60b, p. 1112 (Abstract \# 140).

Pegoraro, L. F., \& Barrack, G. 1987, 'A comparison of bond strengths of adhesive cast restorations using different designs, bonding agents, and luting resins'. J Prosthet Dent, vol. 57, no. 2, pp. 133-8.

Peregrina, A., Schorr, B. L., Eick, J. D., Robinson, S. J., \& Feil, P. H. 1992, 'Measurement of oxide adherence to silver-free high-palladium alloys'. Int $J$ Prosthodont, vol. 5, no. 2, pp. 173-8.

Peterson, E. A. d., Phillips, R. W., \& Swartz, M. L. 1966, 'A comparison of the physical properties of four restorative resins'. J Am Dent Assoc, vol. 73, no. 6, pp. 1324-36.

Peutzfeldt, A., \& Asmussen, E. 1992, 'Modulus of resilience as predictor for clinical wear of restorative resins'. Dent Mater, vol. 8, no. 3, pp. 146-8.

Peutzfeldt, A. 1997, 'Resin composites in dentistry: the monomer systems'. Eur J Oral Sci, vol. 105, no. 2, pp. 97-116.

Phillips, R. W. 1981, 'Symposium on composite resins in dentistry'. Dent Clin North Am, vol. 25 , no. 2 , pp. 209-18.

Phillips, R. W. 1988, 'Bonding agents and adhesives'. Adv Dent Res, vol. 2, no. 1, pp. $150-4$.

Phrukkanon, S., Burrow, M. F., \& Tyas, M. J. 1998, 'Effect of cross-sectional surface area on bond strengths between resin and dentin'. Dent Mater, vol. 14, no. 2, pp. 120-8.

Pröbster, B., \& Henrich, G. M. 1997, '11-year follow-up study of resin-bonded fixed partial dentures'. Int J Prosthodont, vol. 10, no. 3, pp. 259-68.

Rabinowitz, S., \& Beardmore, P. 1974, 'Cyclic deformation and fracture of polymers'. $J$ Mater Sci, vol. 9, pp. 81-99.

Raptis, C. N., Fan, P. L., \& Powers, J. M. 1979, 'Properties of microfilled and visible light-cured composite resins'. J Am Dent Assoc, vol. 99, no. 4, pp. 631-3. 
Re, G. J., Kaiser, D. A., Malone, W. F., \& Garcia-Godoy, F. 1988, 'Shear bond strengths and scanning electron microscope evaluation of three different retentive methods for resin-bonded retainers'. J Prosthet Dent, vol. 59, no. 5, pp. 568-73.

Reeh, E. S., Douglas, W. H., \& Messer, H. H. 1989, 'Stiffness of endodontically-treated teeth related to restoration technique'. J Dent Res, vol. 68, no. 11, pp. 1540-4.

Richardson, M. O.W. 1989, 'Damage assesment in fatigue of reinforced structural composites'. In Advances in fatigue science and technology, eds C. Moura Branco \& L. Guerra Rossa., Kluwer Academic Publishers, Dordrecht, Netherlands, pp. 677-96.

Ritter, J. E. 1995, 'Critique of test methods for lifetime predictions'. Dent Mater, vol. 11, no. 2, pp. 147-51.

Rodford, R. 1986, 'The development of high impact strength denture-base materials'. $J$ Dent, vol. 14, no. 5, pp. 214-7.

Rosenblum, M. A., \& Schulman, A. 1997, 'A review of all-ceramic restorations'. J Am Dent Assoc, vol. 128, no. 3, pp. 297-307.

Rueggeberg, F. A. 1991, 'Substrate for adhesion testing to tooth structure - review of the literature'. Dent Mater, vol. 7, no. 1, pp. 2-10.

Rueggeberg, F. A., Caughman, W. F., Curtis, J. W., Jr., \& Davis, H. C. 1993, 'Factors affecting cure at depths within light-activated resin composites'. Am J Dent, vol. 6, no. 2, pp. 91-5.

Ruffino, A. R. 1985, 'Effect of steel strengtheners on fracture resistance of the acrylic resin complete denture base'. J Prosthet Dent, vol. 54, no. 1, pp. 75-8.

Ruyter, I. E., \& Svendsen, S. A. 1978, 'Remaining methacrylate groups in composite restorative materials'. Acta Odontol Scand, vol. 36, no. 2, pp. 75-82.

Ruyter, I. E., \& Øysæd, H. 1982a, 'Conversion in denture base polymers'. J Biomed Mater Res, vol. 16, no. 5, pp. 741-54.

Ruyter, I. E., \& Øysæd, H. 1982b, 'Conversion in different depths of ultraviolet and visible light activated composite materials'. Acta Odontol Scand, vol. 40, no. 3, pp. 179 92.

Ruyter, I. E., \& Øysæd, H. 1987, 'Composites for use in posterior teeth: composition and conversion'. J Biomed Mater Res, vol. 21, no. 1, pp. 11-23.

Ruyter, I. E., \& Sjøvik Kleven, I. J. 1987, 'Monomers and filler content of resin-based crown and bridge materials'. Dent Mater, vol. 3, no. 6, pp. 315-21. 
Saito, C., Sakai, Y., Node, H., \& Fusayama, T. 1976, 'Adhesion of polycarboxylate cements to dental casting alloys'. J Prosthet Dent, vol. 35, no. 5, pp. 543-8.

Sakaguchi, R. L., Douglas, W. H., \& Peters, M. C. 1992, 'Curing light performance and polymerization of composite restorative materials'. J Dent, vol. 20, no. 3, pp. 183-8.

Schneider, W., Powers, J. M., \& Pierpont, H. P. 1992, 'Bond strength of composites to etched and silica-coated porcelain fusing alloys'. Dent Mater, vol. 8, no. 3, pp. 211-5.

Schreiber, C. K. 1971, 'Polymethylmethacrylate reinforced with carbon fibres'. Br Dent J, vol. 130 , no. 1 , pp. 29-30.

Shen, G., \& Torres, T. 1985, 'The resin bonded cast mesh bridge'. Quintessence Dent Technol, vol. 9, no. 6, pp. 381-5.

Shortall, A. C., Harrington, E., \& Wilson, H. J. 1995, 'Light curing unit effectiveness assessed by dental radiometers'. $J$ Dent, vol. 23 , no. 4 , pp. 227-32.

Shortall, A., \& Harrington, E. 1996, 'Guidelines for the selection, use, and maintenance of visible light activation units'. Br Dent $J$, vol. 181, no. 10, pp. 383-7.

Shue, S. L., Nicholls, J. I., \& Townsend, J. D. 1987, 'The effect of metal retentive designs on resin veneer retention'. J Prosthet Dent, vol. 58, no. 3, pp. 297-305.

Silux Plus Technical Profile 1998, 3M Dental Products - Europe, Boulevard de l'Oise, 95029, Cergy Pontoise Cedex, France

Silva e Souza Junior, M. H., Retief, D. H., Russell, C. M., \& Denys, F. R. 1993, 'Shear bond strength and microleakage of All-Bond'. Am J Dent, vol. 6, no. 3, pp. 148-54.

Smith, L. T., Powers, J. M., \& Ladd, D. 1992, 'Mechanical properties of new denture resins polymerized by visible light, heat, and microwave energy'. Int J Prosthodont, vol. 5, no. 4, pp. 315-20.

Smith, L. T., \& Powers, J. M. 1992, 'Relative fit of new denture resins polymerized by heat, light and microwave energy'. Am J Dent, vol. 5, no. 3, pp. 140-2.

Smith, R. M., Barrett, M. G., Gardner, W. A., Marshall, T., McLean, M. J., McMichael, D. W., Yerbury, P. J., \& Rawls, H. R. 1993, 'Effect of environmental stress and surface treatment on resin-to-metal bonding'. Am J Dent, vol. 6, no. 3, pp. 111-5.

Söderholm, K. J. 1981, 'Degradation of glass filler in experimental composites'. J Dent Res, vol. 60, no. 11, pp. 1867-75.

Söderholm, K. J. 1983, 'Leaking of fillers in dental composites'. J Dent Res, vol. 62, no. 2, pp. 126-30. 
Söderholm, K. J., Zigan, M., Ragan, M., Fischlschweiger, W., \& Bergman, M. 1984, 'Hydrolytic degradation of dental composites'. J Dent Res, vol. 63, no. 10, pp. 1248-54.

Söderholm, K. J. 1984a, 'Influence of silane treatment and filler fraction on thermal expansion of composite resins'. J Dent Res, vol. 63, no. 11, pp. 1321-6.

Söderholm, K. J. 1984b, 'Water sorption in a bis(GMA)/TEGDMA resin'. J Biomed Mater Res, vol. 18, no. 3, pp. 271-9.

Söderholm, K. J., \& Shang, S. W. 1993, 'Molecular orientation of silane at the surface of colloidal silica'. J Dent Res, vol. 72, no. 6, pp. 1050-4.

Soh, G., \& Selwyn, M. J. 1992, 'An evaluation of exposure time and temperature in the thermocycling of dental restorative materials'. Clin Mater, vol. 9, no. 1, pp. 37-41.

Souza, M. H., Retief, D. H., Russell, C. M., \& Denys, F. R. 1994, 'Laboratory evaluation of phosphate ester bonding agents'. Am J Dent, vol. 7, no. 2, pp. 67-73.

Stafford, G. D., \& Smith, D. C. 1970, 'Flexural fatigue tests of some denture base polymers'. Br Dent J, vol. 128, no. 9, pp. 442-5.

Stafford, G. D., \& Brooks, S. C. 1985, 'The loss of residual monomer from acrylic orthodontic resins'. Dent Mater, vol. 1, no. 4, pp. 135-8.

Staninec, M., \& Holt, M. 1988, 'Bonding of amalgam to tooth structure: tensile adhesion and microleakage tests'. J Prosthet Dent, vol. 59, no. 4, pp. 397-402.

Staninec, M., \& Kawakami, M. 1993, 'Adhesion and microleakage tests of a new dentin bonding system'. Dent Mater, vol. 9, no. 3, pp. 204-8.

Stinchcomb, W. W., Reifsnider, K. L., Marcus, L. A., \& Williams, R. S. 1975, 'Effects of Frequency on the Mechanical Response of Two Composite Materials to Fatigue Loads', in Fatigue of composite materials, ed R. Talreja, American Society for Testing and Materials, Philadelphia, USA, pp. 115-29.

Stokes, A. N., \& Tidmarsh, B. G. 1987, 'Porous metal coating--a microretentive alternative to etched metal prostheses'. Quintessence Int, vol. 18, no. 10, pp. 675-8.

Suzuki, S., \& Leinfelder, K. F. 1993, 'Localized wear and marginal integrity of posterior resin composites'. Am J Dent, vol. 6, no. 4, pp. 199-203.

Suzuki, S., Suzuki, S. H., \& Cox, C. F. 1996, 'Evaluating the antagonistic wear of restorative materials when placed against human enamel'. J Am Dent Assoc, vol. 127, no. 1 , pp. 74-80. 
Swartz, M. L., Phillips, R. W., \& Rhodes, B. 1983, 'Visible light-activated resins--depth of cure'. J Am Dent Assoc, vol. 106, no. 5, pp. 634-7.

Takahashi, Y., Chai, J., \& Kawaguchi, M. 1999, 'Equilibrium strengths of denture polymers subjected to long-term water immersion'. Int J Prosthodont, vol. 12, no. 4, pp. 348-52.

Takagi, K., Fujimatsu, H., Usami, H., \& Ogasawara, S. 1996, 'Adhesion between high strength and high modulus polyethylene fibers by use of polyethylene gel as an adhesive'. J Adhes Sci Technol, vol. 10, no.9, pp. 869-82.

Taleghani, M., Leinfelder, K. F., \& Taleghani, A. M. 1987, 'An alternative to cast etched retainers'. J Prosthet Dent, vol. 58, no. 4, pp. 424-8.

Talib, R. 1993, 'Dental composites: a review'. Journal of Nihon University School of Dentistry, vol. 35, no. 3, pp. 161-70.

Tanaka, T., Atsuta, M., Uchiyama, Y., Nakabayashi, N., \& Masuhara, E. 1978, 'Spherical powder for retaining thermosetting acrylic resin veneers'. J Prosthet Dent, vol. 39, no. 3, pp. 295-303.

Tanaka, T., Nagata, K., Takeyama, M., Atsuta, M., Nakabayashi, N., \& Masuhara, E. 1981, '4-META opaque resin--a new resin strongly adhesive to nickel-chromium alloy'. J Dent Res, vol. 60, no. 9, pp. 1697-706.

Tanaka, T., Fujiyama, E., Shimizu, H., Takaki, A., \& Atsuta, M. 1986, 'Surface treatment of nonprecious alloys for adhesion-fixed partial dentures'. J Prosthet Dent, vol. 55, no. 4, pp. 456-62.

Tanaka, T., Atsuta, M., Nakabayashi, N., \& Masuhara, E. 1988, 'Surface treatment of gold alloys for adhesion'. J Prosthet Dent, vol. 60, no. 3, pp. 271-9.

Tanaka, K., Taira, M., Shintani, H., Wakasa, K., \& Yamaki, M. 1991, 'Residual monomers (TEGDMA and Bis-GMA) of a set visible-light-cured dental composite resin when immersed in water'. J Oral Rehabil, vol. 18, no. 4, pp. 353-62.

Tanaka, T., Kamada, K., Matsumura, H., \& Atsuta, M. 1995, 'A comparison of water temperatures for thermocycling of metal-bonded resin specimens'. J Prosthet Dent, vol. 74 , no. 4 , pp. 345-9.

Tarumi, H., Imazato, S., Ehara, A., Kato, S., Ebi, N., \& Ebisu, S. 1999, 'Post-irradiation polymerization of composites containing bis-GMA and TEGDMA'. Dent Mater, vol. 15 , no. 4 , pp. $238-42$.

Thompson, V. P., Grolman, K. H., \& Liao, R. 1985, 'Bonding of adhesive resins to various nonprecious alloys'. J Dent Res, vol. 64, p. 314 (Abstract \# 1258). 
Toparli, M., \& Aksoy, T. 1998, 'Fracture toughness determination of composite resin and dentin/composite resin adhesive interfaces by laboratory testing and finite element models'. Dent Mater, vol. 14, no. 4, pp. 287-93.

Tortopidis, D., Lyons, M. F., Baxendale, R. H., \& Gilmour, W. H. 1998, 'The variability of bite force measurement between sessions, in different positions within the dental arch'. J Oral Rehabil, vol. 25, no. 9, pp. 681-6.

Tulunoğlu, I. F., \& Oktemer, M. 1997, 'Tensile strength and microleakage of the bond between a nickel-chromium alloy and a visible light-cured resin composite: effect of 4META, silicoating, and bead retention'. Quintessence Int, vol. 28, no. 7, pp. 447-51.

Uchiyama, Y. 1986, 'Adhesion in prosthetic restorations', in Proceedings of the International Symposium on Adhesive Prosthodontics, eds L. Gettleman, M. M. A. Vrijhoef, \& Y. Uchiyama, Chicago: Academy of Dental Materials, Amsterdam, Netherlands, pp. 21-31.

Unterbrink, G. L., \& Muessner, R. 1995, 'Influence of light intensity on two restorative systems'. J Dent, vol. 23, no. 3, pp. 183-9.

Uzun, G., Hersek, N., \& Tinçer, T. 1999, 'Effect of five woven fiber reinforcements on the impact and transverse strength of a denture base resin'. J Prosthet Dent, vol. 81, no. 5, pp. 616-20.

Vaidyanathan, J., Vaidyanathan, T. K., Wang, Y., \& Viswanadhan, T. 1992, 'Thermoanalytical characterization of visible light cure dental composites'. J Oral Rehabil, vol. 19, no. 1, pp. 49-64.

Vallittu, P. K. 1993a, 'Comparison of two different silane compounds used for improving adhesion between fibres and acrylic denture base material'. J Oral Rehabil, vol. 20, no. 5, pp. 533-9.

Vallittu, P. K. 1993b, 'Effect of some properties of metal strengtheners on the fracture resistance of acrylic denture base material construction'. J Oral Rehabil, vol. 20, no. 3, pp. 241-8.

Vallittu, P. K., Lassila, V. P., \& Lappalainen, R. 1994a, 'Acrylic resin-fiber composite-Part I: The effect of fiber concentration on fracture resistance'. J Prosthet Dent, vol. 71, no. 6, pp. 607-12.

Vallittu, P. K., Lassila, V. P., \& Lappalainen, R. 1994b, 'Transverse strength and fatigue of denture acrylic-glass fiber composite'. Dent Mater, vol. 10, no. 2, pp. 116-21.

Vallittu, P. K. 1995, 'A review of methods used to reinforce polymethyl methacrylate resin'. J Prosthodont, vol. 4, no. 3, pp. 183-7. 
Vallittu, P. K., Vojtkova, H., \& Lassila, V. P. 1995a, 'Impact strength of denture polymethyl methacrylate reinforced with continuous glass fibers or metal wire'. Acta Odontol Scand, vol. 53, no. 6, pp. 392-6.

Vallittu, P. K., Miettinen, V., \& Alakuijala, P. 1995b, 'Residual monomer content and its release into water from denture base materials'. Dent Mater, vol. 11, no. 6, pp. 338-42.

Vallittu, P. K. 1996a, 'A review of fiber-reinforced denture base resins'. J Prosthodont, vol. 5 , no. 4 , pp. $270-6$.

Vallittu, P. K. 1996b, 'Comparison of the in vitro fatigue resistance of an acrylic resin removable partial denture reinforced with continuous glass fibers or metal wires'. $J$ Prosthodont, vol. 5, no. 2, pp. 115-21.

Vallittu, P. K., \& Narva, K. 1997, 'Impact strength of a modified continuous glass fiber-poly(methyl methacrylate)'. Int J Prosthodont, vol. 10, no. 2, pp. 142-8.

Vallittu, P. K., Ruyter, I. E., \& Ekstrand, K. 1998, 'Effect of water storage on the flexural properties of E-glass and silica fiber acrylic resin composite'. Int J Prosthodont, vol. 11, no. 4 , pp. 340-50.

Vallittu, P. K. 1999a, 'Flexural properties of acrylic resin polymers reinforced with unidirectional and woven glass fibers'. J Prosthet Dent, vol. 81, no. 3, pp. 318-26.

Vallittu, P. K. 1999b, 'Prosthodontic treatment with a glass fiber-reinforced resin-bonded fixed partial denture: A clinical report'. J Prosthet Dent, vol. 82, no. 2, pp. 132-5.

Vallittu, P. K. 1999c, 'Unpolymerized surface layer of autopolymerizing polymethyl methacrylate resin'. J Oral Rehabil, vol. 26, no. 3, pp. 208-12.

Van Noort, R. 1994, 'Clinical relevance of laboratory studies on dental materials: strength determination--a personal view'. J Dent, vol. 22, no. Suppl 1, pp. S4-8.

Van Noort, R., Noroozi, S., Howard, I. C., \& Cardew, G. 1989, 'A critique of bond strength measurements'. J Dent, vol. 17, no. 2, pp. 61-7.

Van Noort, R., Cardew, G. E., Howard, I. C., \& Noroozi, S. 1991, 'The effect of local interfacial geometry on the measurement of the tensile bond strength to dentin'. J Dent Res, vol. 70, no. 5, pp. 889-93.

Vankerckhoven, H., Lambrechts, P., van Beylen, M., \& Vanherle, G. 1981, 'Characterization of composite resins by NMR and TEM'. J Dent Res, vol. 60, no. 12, pp. 1957-65. 
Vargas, M. A., Cobb, D. S., \& Schmit, J. L. 1998, 'Polymerization of composite resins: argon laser vs conventional light'. Oper Dent, vol. 23, no. 2, pp. 87-93.

Versluis, A., Douglas, W. H., \& Sakaguchi, R. L. 1996, 'Thermal expansion coefficient of dental composites measured with strain gauges'. Dent Mater, vol. 12, no. 5, pp. 290 4.

Vijayaraghavan, T. V., \& Hsiao, J. 1994, 'Flexural behavior of visible light-cured composites as a function of temperature under water immersion test conditions'. Dent Mater, vol. 10, no. 6, pp. 347-52.

Vojvodic, D., Jerolimov, V., Celebic, A., \& Catovic, A. 1999, 'Bond strengths of silicoated and acrylic resin bonding systems to metal'. J Prosthet Dent, vol. 81, no. 1, pp. 1-6.

Von Fraunhofer, J. A., Razavi, R., \& Khan, Z. 1988, 'Wear characteristics of highstrength denture teeth'. J Prosthet Dent, vol. 59, no. 2, pp. 173-5.

Wada, T. 1986, 'Development of a new adhesive material and its properties', in Proceedings of the International Symposium on Adhesive Prosthodontics, eds L. Gettleman, M. M. A. Vrijhoef, \& Y. Uchiyama, Chicago: Academy of Dental Materials, Amsterdam, Netherlands, pp. 9-18.

Wassell, R. W., McCabe, J. F., \& Walls, A. W. 1992, 'Subsurface deformation associated with hardness measurements of composites'. Dent Mater, vol. 8, no. 4, pp. 218-23.

Watts, D. C., McNaughton, V., \& Grant, A. A. 1986, 'The development of surface hardness in visible light-cured posterior composites'. J Dent, vol. 14, no. 4, pp. 169-74.

Watts, D. C., Amer, O. M., \& Combe, E. C. 1987, 'Surface hardness development in light-cured composites'. Dent Mater, vol. 3, no. 5, pp. 265-9.

Watson, R. M. 1986, ' Partial dentures', in A companion to dental studies: volume 3: Clinical Dentistry, eds A. H. R. Rowe, A. G. Alexander \& R. B. Johns, Class Publishing, London, England, pp. 782-880.

Whitman, D. J., McKinney, J. E., Hinman, R. W., Hesby, R. A., \& Pelleu, G. B., Jr. 1987 , 'In vitro wear rates of three types of commercial denture tooth materials'. $J$ Prosthet Dent, vol. 57, no. 2, pp. 243-6.

Wictorin, L., Oilo, G., \& Ohlson, N. G. 1989, 'Retentive strength of dental composite to metal surfaces'. Acta Odontol Scand, vol. 47, no. 3, pp. 185-91.

Willems, G., Lambrechts, P., Braem, M., Celis, J. P., \& Vanherle, G. 1992, 'A classification of dental composites according to their morphological and mechanical characteristics'. Dent Mater, vol. 8, no. 5, pp. 310-9. 
Willems, G., Lambrechts, P., Braem, M., \& Vanherle, G. 1993, 'Composite resins in the 21st century'. Quintessence Int, vol. 24, no. 9, pp. 641-58.

Williams, P. T., \& Johnson, L. N. 1993, 'Composite resin restoratives revisited'. J Can Dent Assoc, vol. 59, no. 6, pp. 538-43.

Williams, V. D., Diaz-Amold, A. M., \& Fotos, P. G. 1994, 'Disinfectant storage media and composite/metal bond strength'. Am J Dent, vol. 7, no. 4, pp. 184-6.

Wiltshire, W. A. 1986, 'Tensile bond strengths of various alloy surface treatments for resin bonded bridges'. Quintessence Dent Technol, vol. 10, no. 4, pp. 227-32.

Wiskott, H. W., Nicholls, J. I., \& Belser, U. C. 1995, 'Stress fatigue: basic principles and prosthodontic implications'. Int J Prosthodont, vol. 8, no. 2, pp. 105-16.

Wu, W., \& McKinney, J. E. 1982, 'Influence of chemicals on wear of dental composites'. $J$ Dent Res, vol. 61, no. 10, pp. 1180-3.

Yamamoto, M., \& Takahashi, H. 1995, 'Tensile fatigue strength of light cure composite resins for posterior teeth'. Dent Mater J, vol. 14, no. 2, pp. 175-84.

Yap, U. J., Stokes, A. N., \& Pearson, G. J. 1994, 'Concepts of adhesion--a review'. N Z Dent J, vol. 90, no. 401, pp. 91-7.

Yazdanie, N., \& Mahood, M. 1985, 'Carbon fiber acrylic resin composite: an investigation of transverse strength'. J Prosthet Dent, vol. 54, no. 4, pp. 543-7.

Yoshida, K., Matsumura, H., \& Atsuta, M. 1990, 'Monomer composition and bond strength of light-cured 4-META opaque resin'. J Dent Res, vol. 69, no. 3, pp. 849-51.

Yoshida, K., Matsumura, H., Tanaka, T., \& Atsuta, M. 1992, 'The relationship between monomer composition and physical properties of light-cured opaque resin'. Dent Mater, vol. 8, no. 2, pp. 137-9.

Yoshida, K., Taira, Y., Matsumura, H., \& Atsuta, M. 1993, 'Effect of adhesive metal primers on bonding a prosthetic composite resin to metals'. J Prosthet Dent, vol. 69, no. 4, pp. 357-62.

Yoshida, K., Sawase, T., Watanabe, I., \& Atsuta, M. 1995, 'Shear bond strengths of four resin cements to cobalt-chromium alloy'. Am J Dent, vol. 8, no. 6, pp. 285-8.

Yoshida, K., Kamada, K., Tanagawa, M., \& Atsuta, M. 1996, 'Shear bond strengths of three resin cements used with three adhesive primers for metal'. J Prosthet Dent, vol. 75 , no. 3, pp. 254-61. 
Yoshida, K., Taira, Y., Sawase, T., \& Atsuta, M. 1997, 'Effects of adhesive primers on bond strength of self-curing resin to cobalt-chromium alloy'. J Prosthet Dent, vol. 77, no. 6, pp. 617-20.

Yoshida, K., Kamada, K., \& Atsuta, M. 1999, 'Adhesive primers for bonding cobaltchromium alloy to resin'. J Oral Rehabil, vol. 26, no. 6, pp. 475-8.

Z100 Product Information 1997, 3M Dental Products - Europe, Boulevard de l'Oise, 95029, Cergy Pontoise Cedex, France

Zhao, D., Botsis, J., \& Drummond, J. L. 1997, 'Fracture studies of selected dental restorative composites'. Dent Mater, vol. 13, no. 3, pp. 198-207. 
Alicylic spiroorthocarbonates : SOCs

Biphenyldimethacrylate : BPDM

Bis-ethyl methacrylate : Bis-EMA

Bis-glycidyl methacrylate : Bis-GMA

Bis-methacrylate : Bis-MA

Ethylene glycol dimethacrylate : EGDMA

4-methacryloxyethyl trimellitate anhydride : 4-META

3-methacryloxypropyl trimethoxysilane : MAPM

10-methacryloyloxy-decyl-dihydrogen phosphate : 10-MDP

Methylmethacrylate : MMA

Poly(methylmethacrylate) : PMMA

Triethylene glycol dimethacrylate : TEGDMA

Urethane dimethacrylate : UEDMA

Appendix I: Abbreviations 


\begin{tabular}{|c|c|c|c|c|}
\hline Equipment & Model & Number & Remarks & Manufacturer \\
\hline Air abrasion unit & Sandstorm & 240027 & $50 \mu$ aluminium oxide at $80 \mathrm{psi}$ & $\begin{array}{l}\text { Chapelin and Jacobs Ltd, 1Four Seasons Cres., Surrey, } \\
\text { England }\end{array}$ \\
\hline Ultrasonic cleaner & Transonic T570 & 8014208 & Frequency $35 \mathrm{KHZ}$ & Lam Lab Serving Science, London, England \\
\hline Specimen Mould & NA & NA & \multirow{3}{*}{$\begin{array}{l}\text { Designed and made for preparation of } \\
\text { similarly dimensioned specimens }\end{array}$} & \multirow{3}{*}{$\begin{array}{l}\text { Biomaterials Dept. EDI } \\
\text { 256, Grays Inn Road, } \\
\text { London WC1, England }\end{array}$} \\
\hline Specimen jig & NA & NA & & \\
\hline Thermo-cycling unit & NA & NA & & \\
\hline \multirow[t]{2}{*}{ Light curing units } & Luxor 4000 & 4031 & 170 watts, Frequency $50-60 \mathrm{HZ}$ & $\begin{array}{l}\text { ICI Pharmaceuticals Division, Macclesfield, Cheshire, } \\
\text { England }\end{array}$ \\
\hline & Unilux AC & $21 / 20059$ & Light curing vessel & Kulzer GMBH,Bereich Dental, Phillip-Reiss Str., Germany. \\
\hline Load testing machine & Instron 4505 & H2266 & Used in 3-point bend testing & Instron Corp., 100 Royal St.,Canton, Mass 02021, USA. \\
\hline Power amplifier & 2707 & 805616 & Part of the fatigue testing set-up & \multirow{2}{*}{$\begin{array}{l}\text { Bruel \& Kjoer, } \\
\text { Copenhagen, Denmark }\end{array}$} \\
\hline Electromagnet & 4812 & 821407 & $\begin{array}{l}\text { Part of the fatigue testing set-up, Current } \\
\text { limit: } 22 \text { amperesrms, Displacement limit: } \\
0.5 \text { inch/volt.sec }\end{array}$ & \\
\hline Load cell & $41 / 8186-03$ & 485750 & $\begin{array}{l}\text { Part of the fatigue testing set-up, } \\
\text { Range: } 0-250 \mathrm{lbs}\end{array}$ & RDP Electronics,London, England. \\
\hline Signal generator & Thandar TG502 & 000444 & $\begin{array}{l}\text { Part of the fatigue testing set-up } 5 \mathrm{MHz} \\
\text { sweep }\end{array}$ & $\begin{array}{l}\text { Thandar Electronics Ltd, London rd, St.Ives, } \\
\text { Huntington, Cambs, PE17, 4HJ }\end{array}$ \\
\hline Light microscope & Wild M400 & 153712 & $\begin{array}{l}\text { Light microscope with vernier controls on } \\
\text { the stage (accurate up to } 0.01 \mathrm{~mm} \text { ) }\end{array}$ & $\begin{array}{l}\text { Wild Heerbrugg Ltd, } \\
\text { CH-9435, Heerbrugg, Switzerland }\end{array}$ \\
\hline Ionalyser & Orion 901 & 1750 & Used to measure the $\mathrm{pH}$ of the solutions & $\begin{array}{l}\text { Quadrachem Labs, Riverside, Forest Rd Business Park, } \\
\text { Forest Row, East Sussex, RH18 5EW }\end{array}$ \\
\hline Sputter coater & S150 A & 53800 & Used to prepare specimens for SEM viewing & Edwards Instruments, Eastbourne, Sussex, England \\
\hline Electron microscope & Cambridge Stereoscan S90 & 9205-B & Accelerating voltage of $15 \mathrm{kV}$ & Cambridge Instruments, Clifton Road, Cambridge, CB1 \\
\hline Analytical balance & Precisa $120 \mathrm{~A}$ & $\mathrm{~J} 67340$ & $\begin{array}{l}\text { Used to weigh specimens during the water } \\
\text { sorption study }\end{array}$ & PAG Oerlicon, Zurich, Switzerland \\
\hline
\end{tabular}

\section{Appendix II: Equipment used}




\begin{tabular}{|c|c|c|c|c|}
\hline $\begin{array}{l}\text { Material } \\
\text { Type }\end{array}$ & Material & Batch Nos. & Remarks & Manufacturer \\
\hline \multirow[t]{4}{*}{$\begin{array}{l}\text { Light Curing } \\
\text { Composite System }\end{array}$} & Silux Plus & $\begin{array}{l}19961030,19961204 \\
19960129,19951129\end{array}$ & Shade: Universal & \multirow[t]{2}{*}{$\begin{array}{l}\text { 3M Dental Products } \\
\text { St.Paul, MN 55144-1000, USA }\end{array}$} \\
\hline & $\mathrm{Z100}$ & $\begin{array}{l}19951218,19950615 \\
19961202,19970124\end{array}$ & Shade: A3 & \\
\hline & Cesead opaque resin & $0025,00008-10$ & Shade:0C2 & \multirow{2}{*}{$\begin{array}{l}\text { Kuraray Co LTD } \\
\text { Kurashiki, } \\
\text { Japan }\end{array}$} \\
\hline & $\begin{array}{l}\text { Cesead opaque } \\
\text { Primer }\end{array}$ & 00039 & $\mathrm{~N} / \mathrm{A}$ & \\
\hline Casting Wax & $\begin{array}{l}\mathrm{C}+\mathrm{J} \text { plain sheet } \\
\text { casting wax }\end{array}$ & & Thickness:0.8mm & \multirow{2}{*}{$\begin{array}{l}\text { Chapelin \& Jacobs LTD } \\
\text { 1, Four seasons crescent, } \\
\text { Limpton Road, } \\
\text { Sutton, Surrey, England. }\end{array}$} \\
\hline Blasting grit & Blast grain & & $\begin{array}{l}\text { Equal mixture of coarse } \\
\text { and extra coarse grit. } \\
\text { Used to remove the } \\
\text { investment from the } \\
\text { castings }\end{array}$ & \\
\hline Aluminum oxide & Strahlmittel Abrasives & & $\begin{array}{l}\text { Aluminum Oxide } 50 \mu \mathrm{m} \\
\text { used to blast the metal } \\
\text { surfaces for bonding }\end{array}$ & $\begin{array}{l}\text { Renfert GmbH, D-78247, Hilzingen, } \\
\text { Germany }\end{array}$ \\
\hline Casting Alloy & $\begin{array}{l}\text { S P Chrome cobalt } \\
\text { Alloy }\end{array}$ & & Ingot wt:5gms & $\begin{array}{l}\text { Bayer PLC, Dental Products Gp. } \\
\text { Pharmaceutical Division } \\
\text { Bayer House,Newbury, } \\
\text { Berkshire, England. }\end{array}$ \\
\hline
\end{tabular}

Appendix III: Materials used 


\section{$\underline{\text { Errata }}$}

Page 3, Line 7: Delete though

Page 11, Line 9: Delete $2^{\text {nd }}$ comma

Page 12, Line 13: Delete a

Page 12, Line 14: Change strength to strengths

Page 15, Line 1: Change of to to

Page 15, Line 2: Delete being

Page 16, Line 5: Delete though

Page 23, Line 17: Change as to that

Page 26, Line 14: Change acknowledge to achieve

Page 26, Line 17: Delete though

Page 26, Line 20: Change the to a

Page 26, Line 21: Change the to a

Page 26, Line 23: Insert on after depending

Page 31, Line 17: Delete that. Change in between to of

Page 32, Line 17: Change dependant to dependent. Delete though

Page 37, Line 18: Insert when after irritant. Insert and after skin,. Delete, after therefore

Page 40, Line 18: Add to after prior

Page 40, Line 19: Change reduce to be reduced

Page 40, Line 20: Add, after regions

Page 41, Line 5: Delete degree

Page 41, Line 15: Change required to requires

Page 41, Line 16: Change burned to burns

Page 41, Line 24: Change attaches to attach

Page 42, Line 20: Insert However before This. Delete though

Page 44, Line 3: Change Its to It has

Page 45, Line 11: Change have to has

Page 49, Line 3: Change an to a

Page 51, Line 8: Insert the after on

Page 57, Line 3: Insert on after depending

Page 57, Line 19: Insert on after depending

Page 58, Line 23: Change has to have

Page 59, Line 22: Change the to that

Page 64, Line 6: Change are to is

Page 65, Line 19: Change disposed to dispensed

Page 81, Legend; Figure 4.7b: Change $4 \mathrm{~mm}$ length to $4 \mathrm{~mm}$ width

Page 94, Line 3: Insert perforated after small

Page 96, Line 2: Insert dry after load cycled

Page 135, Line 8: Insert at one week after obtained

Page 145, Line 8: Change was to were. Delete in

Page 147, Line 14: Insert they after Z100

Reference Harrison, A., Huggett, R., \& Jagger, R. C. change to Harrison, A., Huggett, R., \& Jagger, R. G. 


\section{$\underline{\text { Addenda }}$}

Page 44; Add sentence at end of paragraph 3: This is probably associated with the fact that the dentine surface is always moist whereas metal is a clean uncontaminated surface. In the longer term water uptake will probably have an effect at the metal/adhesive interface.

Page 151; Add sentence at end of paragraph 1: For the purpose of this work, cohesive failure is defined as a vertical failure within the veneering resin and adhesive failure as a failure of the veneering resin along the metal boundary.

Page 63; Replace hypothesis with: The use of differing veneering resins bonded to a metal substructure, will influence the long-term mechanical properties of the combined laminate structure after fatiguing by load and thermo-cycling.

Page 103; Figure 5.3, Page 105; Figure 5.6, Page 109; Figure 5.9, Page 111; Figure 5.12: Change hours to hours ${ }^{1 / 2}$ 